\title{
Effect of repeated MDMA exposure on rat brain and behaviour
}

\author{
by \\ Ross van de Wetering
}

A thesis submitted in fulfilment of the requirements for the degree of Doctor of Philosophy

Victoria University of Wellington, New Zealand 
Table of contents

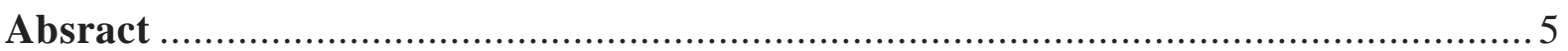

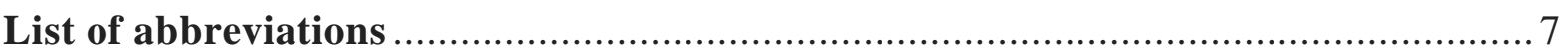

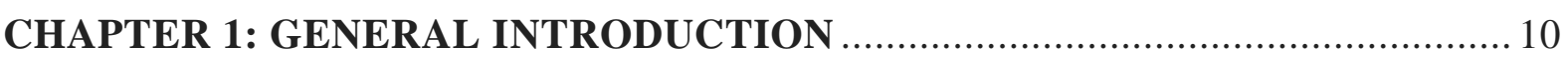

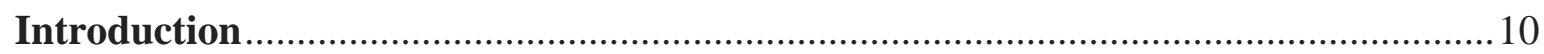

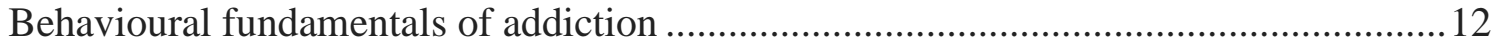

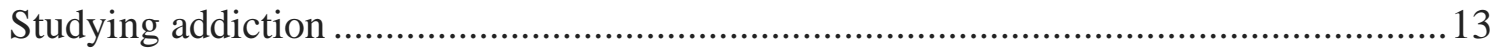

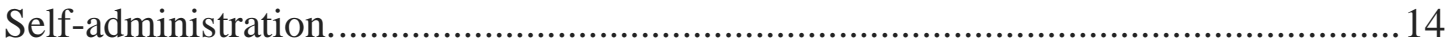

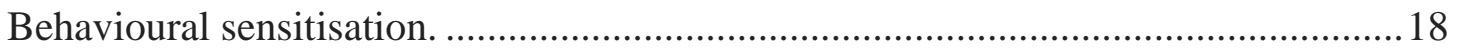

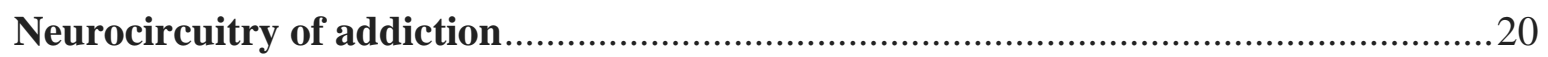

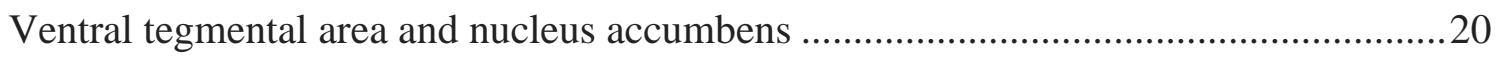

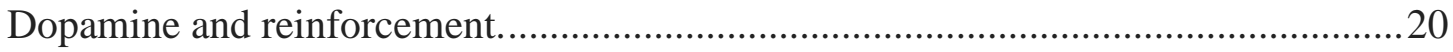

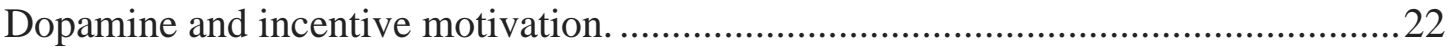

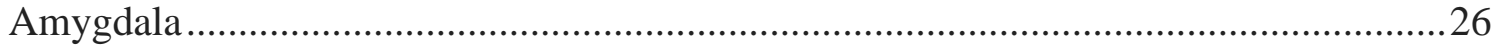

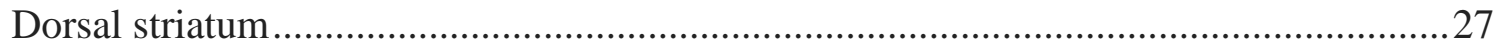

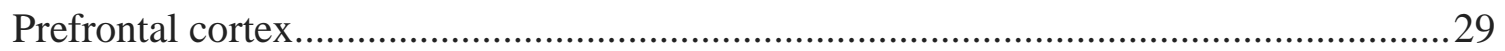

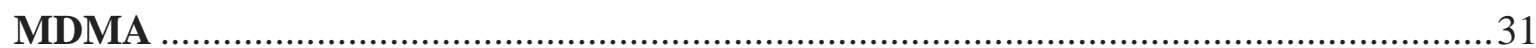

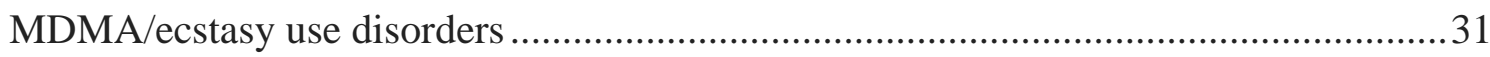

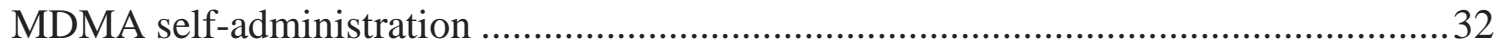

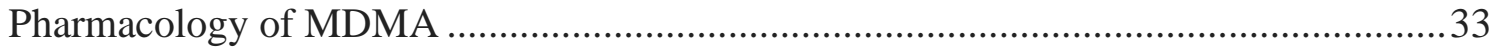

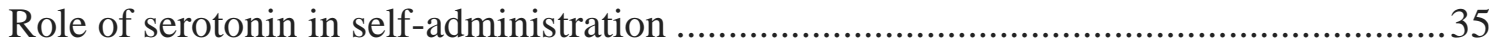

Effect of repeated MDMA exposure on serotonin and dopamine .................................. 37

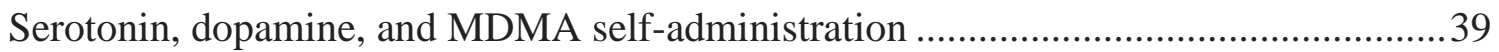

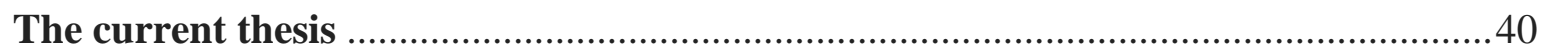

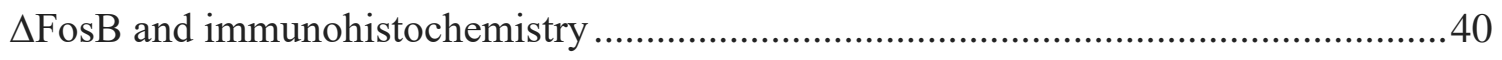

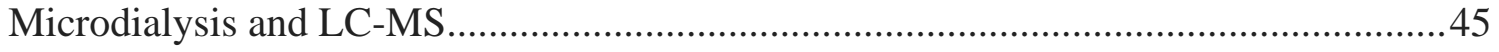

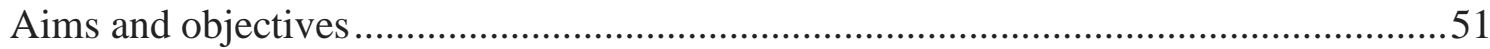

CHAPTER 2: $\triangle$ FOSB AND IMMUNOHISTOCHEMISTRY ............................. 52

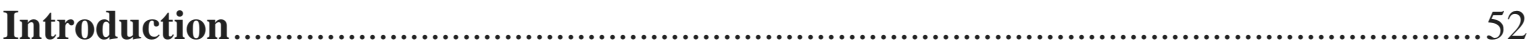

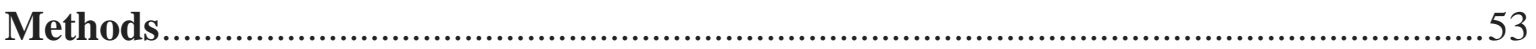

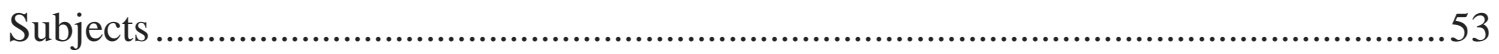

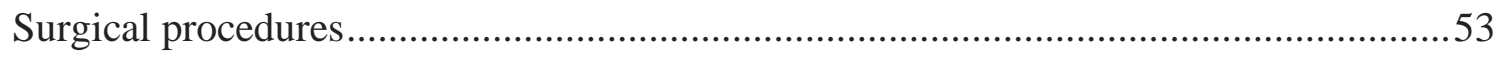

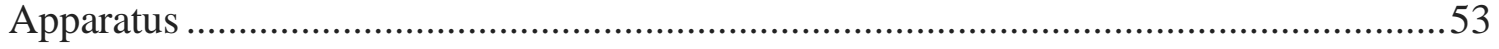




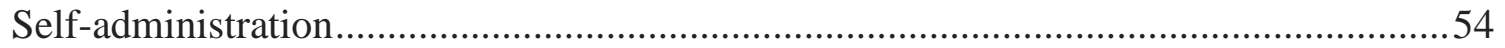

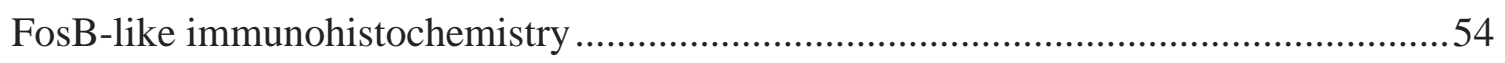

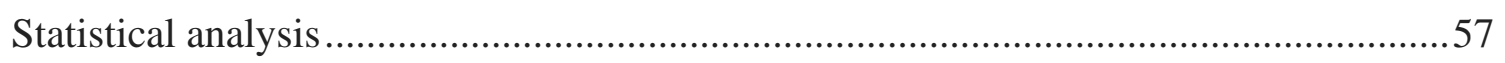

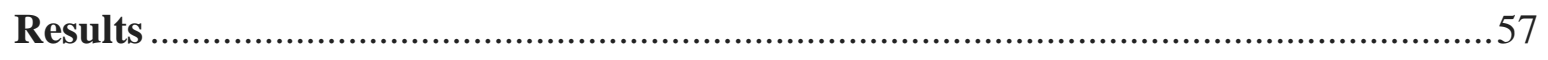

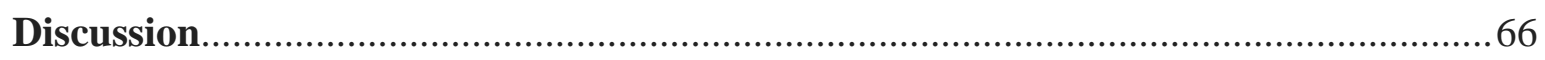

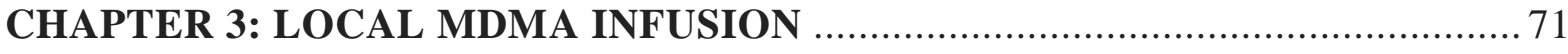

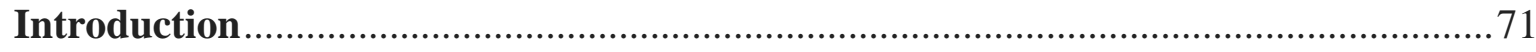

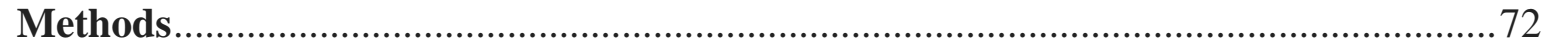

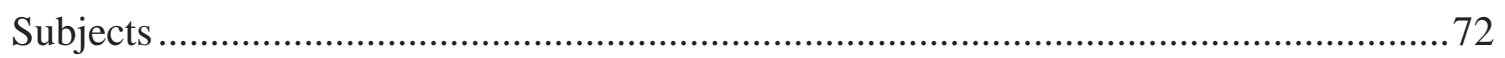

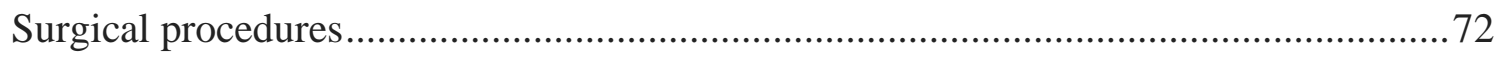

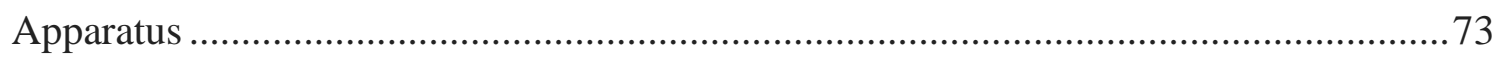

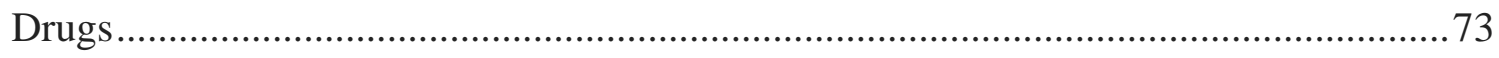

Pretreatment and intra-striatal microinjections ........................................................ 73

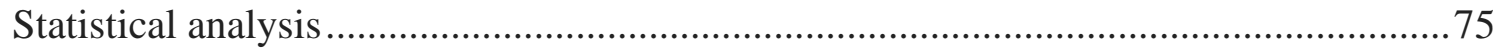

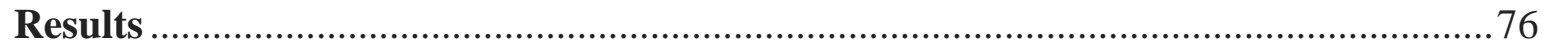

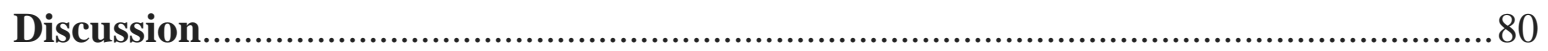

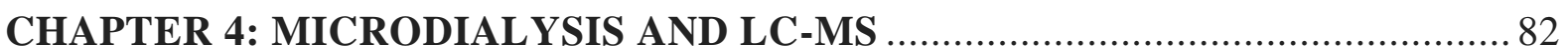

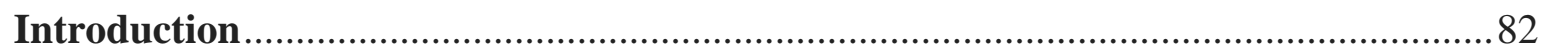

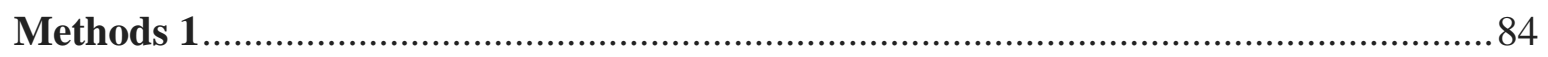

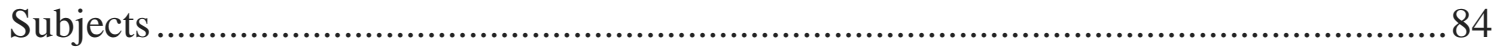

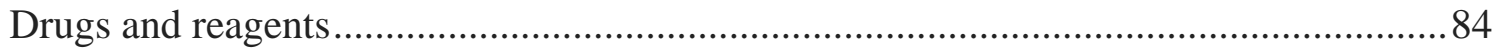

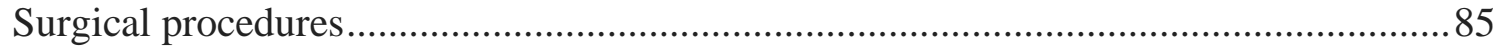

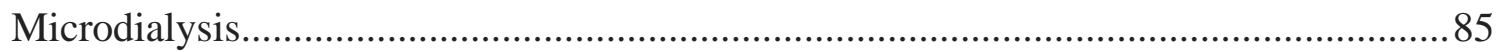

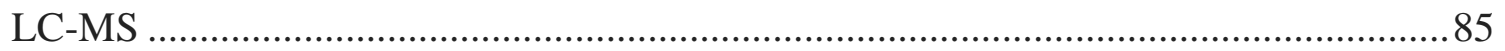

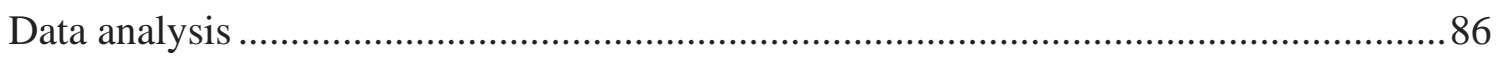

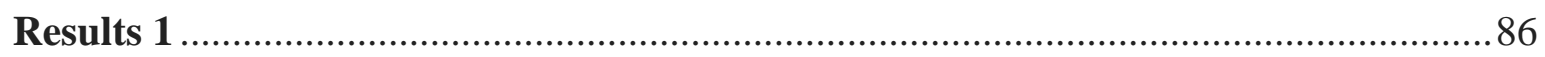

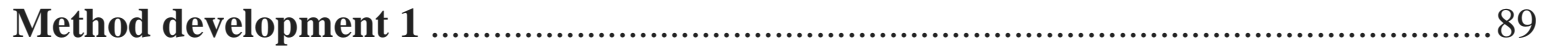

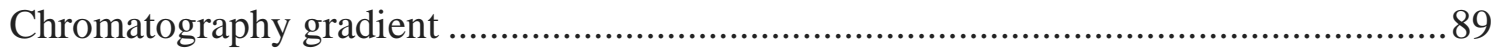

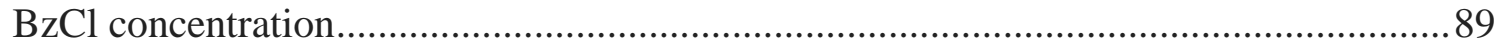

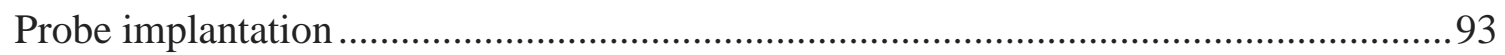

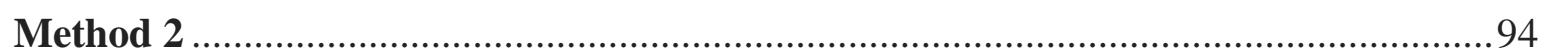

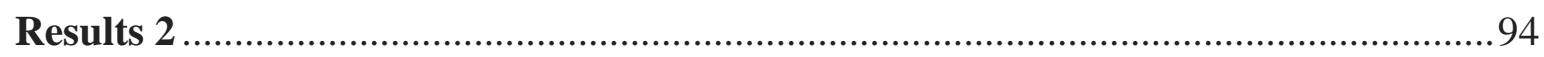




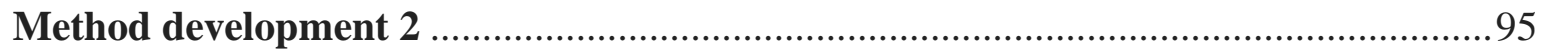

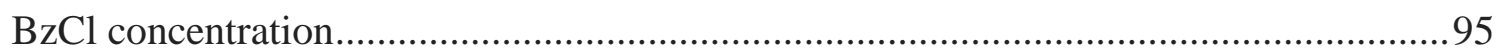

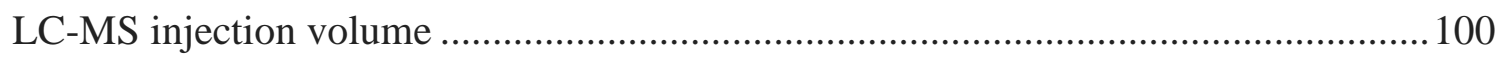

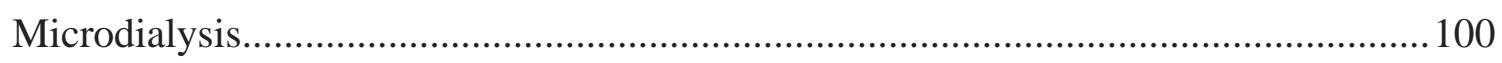

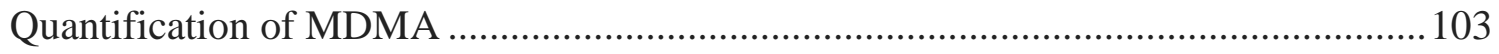

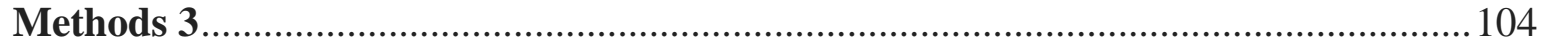

MDMA pretreatment and locomotor sensitisation ................................................... 104

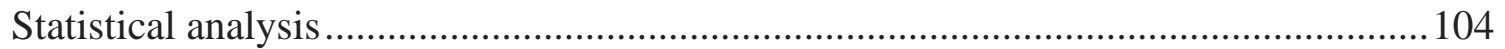

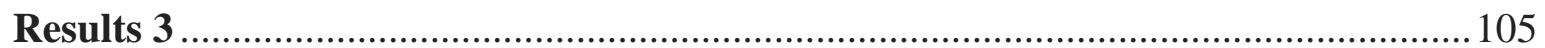

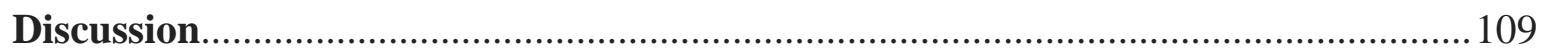

CHAPTER 5: UNTARGETED METABOLOMICS ....................................... 112

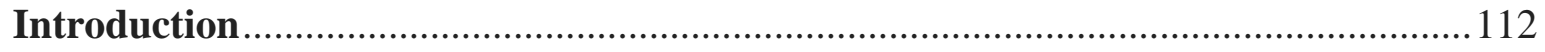

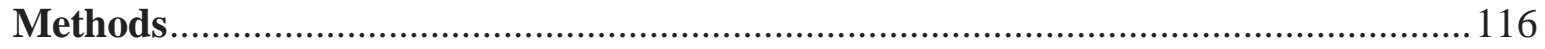

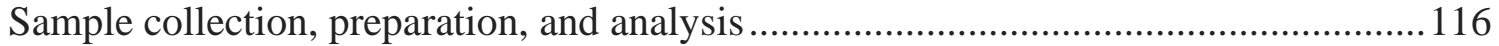

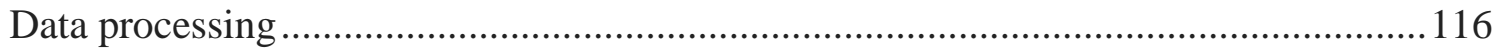

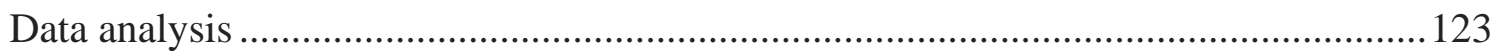

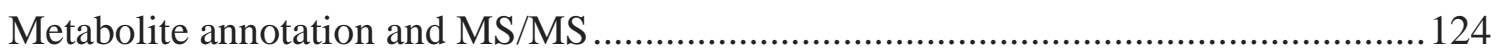

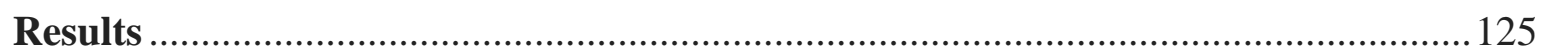

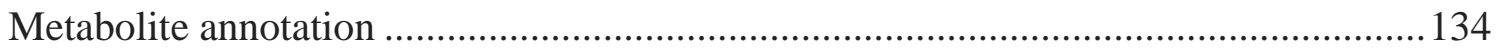

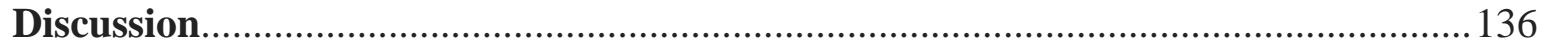

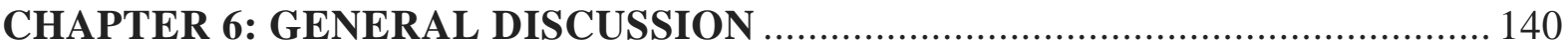

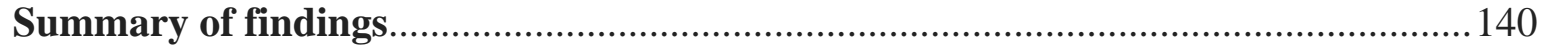

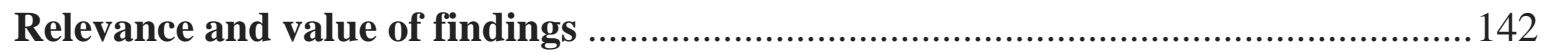

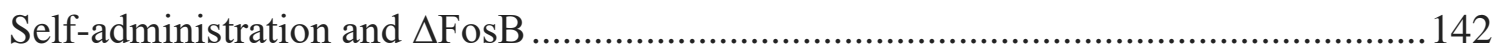

Behavioural sensitisation and the acquisition of self-administration ........................... 144

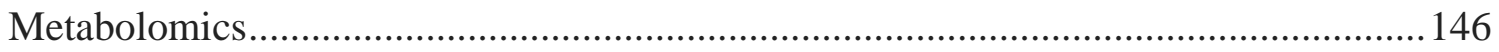

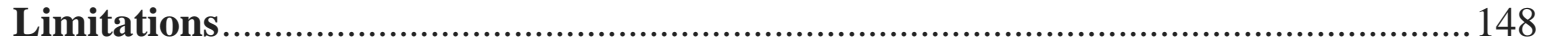

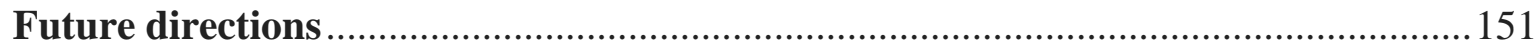

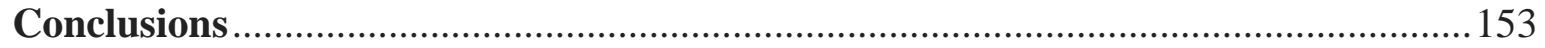

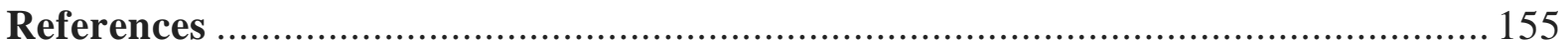

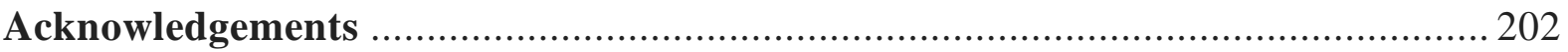




\begin{abstract}
Rationale. $\pm 3,4$-Methylenedioxymethamphetamine (MDMA; 'ecstasy') is a popular recreational drug of abuse. Like other drugs of abuse, a proportion of users develop symptoms that are characteristic of a Substance Use Disorder (SUD). The behavioural and neurobiological consequences of repeated misuse of MDMA are not well understood, however.

Objectives. The purpose of the present thesis was to investigate behaviourally relevant neuroadaptations that develop with repeated MDMA exposure in laboratory rats.

Methods. First, the effect of chronic, long-access (6 hour) self-administration of MDMA on the accumulation of the transcription factor, $\triangle \mathrm{FosB}$, in the nucleus accumbens (core, shell), dorsal striatum (dorsomedial, dorsolateral, ventromedial, ventrolateral), prefrontal cortex (anterior cingulate, prelimbic, infralimbic, orbitofrontal), amygdala (central, basolateral), ventral tegmental area (anterior, posterior), and raphe (dorsal, median) was measured using immunohistochemistry. Second, the behavioural relevance of these findings was determined by examining the effect of bi-lateral intra-striatal (nucleus accumbens, dorsomedial striatum, dorsolateral striatum) microinjections of MDMA (200 $\mu \mathrm{g} / 1 \mu \mathrm{L} / \mathrm{side})$ on the expression of behavioural sensitisation following two days of withdrawal from a regimen of repeated, systemic MDMA exposure (10 mg/kg/day, i.p., for 5 days). Third, a procedure was developed to examine neurochemical correlates of sensitised MDMA-produced behaviour ( 0 , $5,10 \mathrm{mg} / \mathrm{kg}$, i.p.) following the same regimen of repeated MDMA exposure. Samples were collected from the medial striatum using in vivo microdialysis and the extracellular concentrations of serotonin, dopamine, MDMA, and their metabolites were quantified using liquid chromatography coupled with quadrupole time-of-flight (Q-TOF) mass spectrometry. Lastly, a unique untargeted metabolomics procedure was developed to further analyse these microdialysis samples and to identify novel or unexpected metabolites that were relevant to the sensitised behavioural response produced by MDMA.
\end{abstract}

Results. MDMA self-administration produced region-dependant increases in $\Delta \mathrm{FosB}$. Significant increases in $\Delta$ FosB were observed in the nucleus accumbens core, the medial areas of the dorsal striatum, as well as all areas of the prefrontal cortex and amygdala. Small, but significant increases were also observed in the dorsal raphe. Increases were observed in the nucleus accumbens shell and the posterior tail of the ventral tegmental area, but these increases were not significant following statistical correction for multiple comparisons. Acute exposure to MDMA increased locomotor activity only when the drug was infused into the nucleus accumbens. Following repeated systemic exposure, behavioural sensitisation was expressed 
when MDMA was infused into both the nucleus accumbens or the dorsomedial striatum, but not the dorsolateral striatum. Analysis of microdialysates from the medial striatum indicated that behavioural sensitisation was accompanied by small increases in baseline levels of extracellular serotonin and decreased MDMA-produced increases in serotonin, but these changes were not statistically significant. Behavioural sensitisation was also accompanied by increased extracellular concentrations of dopamine at baseline and following acute MDMA exposure, but these data were not statistically analysed due to small sample sizes. MDMAproduced extracellular concentrations of MDMA did not change with repeated exposure. Untargeted metabolomics revealed potential changes in MDMA and dopamine metabolism that might be relevant to the sensitised behavioural response.

Conclusions. The findings of the current research suggest that repeated MDMA exposure results in many of the same neuroadaptations that result from repeated exposure to other drugs of abuse. These included increased $\Delta \mathrm{FosB}$ expression in many brain regions that are relevant to addiction, such as the nucleus accumbens, dorsal striatum, and prefrontal cortex. Dopaminergic mechanisms also appeared to be influenced and were associated with sensitised MDMA-produced behaviour. Surprisingly, serotonergic mechanisms were not significantly impacted by repeated MDMA exposure under the current conditions. Some of the procedures developed in this thesis are unique and may be of value for future research investigating the neurochemical underpinnings of addictive behaviour or other disease states. 


\section{List of abbreviations}

3-MT: 3-Methoxytyramine

5,7-DHT: 5,7-Dihydroxytryptamine

5-HIAA: 5-Hydroxyindoleacetic acid

5-HT: $\quad$ Serotonin

6-OHDA: 6-Hydroxydopamine

aCSF: $\quad$ Artificial cerebrospinal fluid

ADAP: $\quad$ Automated data analysis pipeline

AMPA: $\quad \alpha$-Amino-3-hydroxy-5-methyl-4-isoxazolepropionic acid

ANOVA: $\quad$ Analysis of variance

AP: $\quad$ Anteroposterior

AP-1: $\quad$ Activator protein-1

ARC: $\quad$ Activity-regulated cytoskeleton-associated protein

BLA: $\quad$ Basolateral amygdala

$\mathrm{BzCl}$ Benzoyl chloride

CeA: $\quad$ Central nucleus of the amygdala

CDK5: $\quad$ Cyclin-dependent kinase 5

COMT: $\quad$ Catechol- $O$-methyltransferase

C-P: $\quad$ Caudate-putamen (dorsal striatum)

CPP: $\quad$ Conditioned place preference

CRF: Corticotrophin-releasing factor

DA: Dopamine

DLS: $\quad$ Dorsolateral striatum

DMS: $\quad$ Dorsomedial striatum

DNA: Deoxyribonucleic acid

DOPAC: $\quad 3,4$-Dihydroxyphenylacetic acid

DSM: Diagnostic and Statistical Manual of Mental Disorders

DV: Dorsoventral

EIC: $\quad$ Extracted ion chromatogram

ESI: $\quad$ Electrospray ionisation

FR: $\quad$ Fixed ratio

GABA: Gamma-aminobutyric acid

GC-MS: $\quad$ Gas chromatography-mass spectrometry 
HHA: 3,4-Dihydroxyamphetamine

HHMA: 3,4-Dihydroxymethamphetamine

HMA: 4-Hydroxy-3-methoxyamphetamine

HMMA: 4-Hydroxy-3-methoxymethamphetamine

HPLC: $\quad$ High-performance liquid chromatography

IHC: Immunohistochemistry

i.p.: Intraperitoneal

i.v.: Intravenous

LC-MS: $\quad$ Liquid chromatography-mass spectrometry

LOD: $\quad$ Limit of detection

MDA: 3,4-Methylenedioxyamphetamine

MDMA: 3,4-Methylenedioxymethamphetamine

ML: $\quad$ Mediolateral

mRNA: Messenger ribonucleic acid

MS: $\quad$ Mass spectrometry

MS/MS: $\quad$ Tandem mass spectrometry

MSN: $\quad$ Medium spiny neurons

$\mathrm{m} / \mathrm{z}$ : $\quad$ Mass-to-charge ratio

NAcc: Nucleus accumbens

NE: $\quad$ Norepinephrine (noradrenaline)

NF-kB: $\quad$ Nuclear factor-kappaB

NMR: $\quad$ Nuclear magnetic resonance

OFC: $\quad$ Orbitofrontal cortex

OPLS: $\quad$ Orthogonal partial least squares

OPLS-DA: Orthogonal partial least squares discriminant analysis

PB: $\quad$ Phosphate buffer

PBS: $\quad$ Phosphate-buffered saline

PBST: $\quad$ Phosphate-buffered saline containing $0.3 \%$ Triton X-100

PCA: Principal components analysis

pCPA: $\quad$-Chlorophenylalanine

PFA: $\quad$ Paraformaldehyde

PFC: $\quad$ Prefrontal cortex

PR: $\quad$ Progressive ratio 
PLS: $\quad$ Partial least squares (projection to latent structures)

PLS-DA: $\quad$ Partial least squares discriminant analysis

Q-TOF: $\quad$ Quadrupole time-of-flight

QQQ: $\quad$ Triple quadrupole mass spectrometry

RNA: $\quad$ Ribonucleic acid

RSD: $\quad$ Relative standard deviation

s.c.: $\quad$ Subcutaneous

SD: $\quad$ Standard deviation

SEM: $\quad$ Standard error of the mean

SIMCA: $\quad$ Soft Independent Modelling of Class Analogies

S/N: $\quad$ Signal to noise ratio

SPSS: $\quad$ Statistical Package for Social Sciences

SSRI: $\quad$ Selective serotonin reuptake inhibitor

SUD: $\quad$ Substance use disorder

SUS: $\quad$ Shared and unique structures

TAAR1: $\quad$ Trace amine-associated receptor 1

TIC: $\quad$ Total ion chromatogram

VMAT: Vesicular monoamine transporter

VTA: Ventral tegmental area

RT: $\quad$ Retention time

VIP: $\quad$ Variable importance in projection

VMA: $\quad$ Vanillylmandelic acid 


\section{CHAPTER 1: GENERAL INTRODUCTION}

Parts of this chapter have been adapted with licensed permission from work published in Addiction Biology (van de Wetering \& Schenk, 2019).

\section{Introduction}

Harmful use of tobacco, alcohol, and illicit drugs are some of the leading causes of preventable health loss in New Zealand, collectively accounting for over 130000 years of healthy life lost annually due to premature death, illness, or disability (Ministry of Health, 2013 , 2016). For comparison, that is over four times greater than that caused by breast and prostate cancer combined (Ministry of Health, 2013, 2016). The health issues associated with harmful substance use include overdose, increased risk of physical injury or suicide, various cancers, chronic diseases, and several neuropsychiatric disorders (Ministry of Health, 2013, 2016). The impact of harmful substance use also extends beyond the health of the individual. Relationship and family issues, domestic violence, child neglect, fetal drug exposure, crime, second-hand smoke, difficulty obtaining and maintaining employment, and traffic accidents are all examples of substance-related harm that affects others (Inter-Agency Committee on Drugs, 2015).

Harmful substance use also carries a significant economic burden. A 2016 study estimated that the annual cost of illicit drug use in New Zealand was \$1.8 billion (McFadden Consultancy, 2016). Older estimates on the annual cost of illicit drug use and alcohol use combined were upwards of $\$ 6$ billion (Slack, Nana, Webster, Stokes, \& Wu, 2009), while the annual cost associated with tobacco use was estimated to be as high a $\$ 1.6$ billion (O’Dea \& Thompson, 2007). These estimates include the cost of healthcare-associated with substance use related accidents, injuries, and illnesses, the cost of intervention and rehabilitation programs, the costs to the criminal justice system, and the cost of welfare payments for people who have become incapacitated through substance use (Inter-Agency Committee on Drugs, 2015; McFadden Consultancy, 2016; O’Dea \& Thompson, 2007; Slack et al., 2009).

Although not every instance of drug use is harmful, the health, financial, and societal consequences caused by individuals who develop a harmful pattern of substance use characteristic of addiction are severe. Drug addiction is a chronic, relapsing disorder characterised by compulsive drug-seeking and drug-taking behaviour despite adverse consequences. In the fifth edition of the Diagnostic and Statistical Manual of Mental Disorders 
(DSM-V), drug addiction is clinically defined as a Substance Use Disorder (SUD), which is characterised by 11 diagnostic criteria (Table 1.1) and is measured along a continuum from mild ( 2 - 3 criteria) to severe (6+ criteria; American Psychiatric Association [APA], 2013). In order to develop new strategies for the prevention and treatment of SUDs, a better understanding of the various factors underlying harmful drug use and the development of SUDs are required.

Table 1.1.

\section{DSM-V Substance Use Disorder criteria}

1. The substance is often taken in larger amounts or over a longer period than was intended.

2. Inability to cut down or control substance use despite a desire to do so.

3. A great deal of time is spent in activities necessary to obtain, use, or recover from the substance.

4. Craving, or a strong desire or urge to use the substance.

5. Repeated usage results in a failure to fulfil major role obligations at work, school, or home.

6. Continued usage despite having persistent or recurrent social or interpersonal problems caused or exacerbated by the effects of the substance.

7. Important social, occupational, or recreational activities are given up or reduced because of substance use.

8. Recurrent usage in situations in which it is physically hazardous.

9. Continued usage despite knowledge of having a persistent or recurrent physical or psychological problem that is likely to have been caused or exacerbated by the substance.

10. Tolerance, as evidenced by a need for markedly increased amounts of the substance to achieve the desired effect and/or a markedly diminished effect with continued use of the same amount of the substance.

11. Withdrawal, as evidenced by the emergence of the characteristic group of withdrawal symptoms as the amount of substance in the body decreases and/or the substance is taken to relieve or avoid such withdrawal symptoms.

Source: (APA, 2013). 


\section{Behavioural fundamentals of addiction}

Changes in the reinforcement processes (Skinner, 1938) underlying drug-taking behaviour are an important factor driving the progression of a SUD. Most drugs that are misused produce rewarding subjective effects (e.g. euphoria). This can function as a positive reinforcer, increasing the likelihood of subsequent drug-taking behaviour. However, it is well known that with repeated drug exposure, tolerance to the rewarding effects of many drugs of abuse can quickly develop (Fischman \& Schuster, 1981; Fischman, Schuster, Javaid, Hatano, \& Davis, 1985; Foltin \& Fischman, 1991). Thus, while positive reinforcement may underlie initial, recreational drug-taking behaviour, it does not explain continued drug-use when the rewarding effects of the drug have dramatically decreased due to tolerance.

In this situation, continued drug-taking behaviour may be better explained by negative reinforcement. Repeated drug use can lead to the development of withdrawal symptoms; aversive physical, mental, or emotional states that occur following the cessation of drug intake. These withdrawal symptoms can be avoided or alleviated by further drug-use (Ahmed \& Koob, 2005; Koob, 2013). Thus, even when the drug no longer produces any pleasurable rewarding effects due to tolerance, the removal or avoidance of these aversive withdrawal symptoms can act as a negative reinforcer for continued drug-taking behaviour.

The transition from positive reinforcement to negative reinforcement is well described by the opponent-process theory of addiction/motivation (Koob, Caine, Parsons, Markou, \& Weiss, 1997; Koob \& Le Moal, 2008b; Solomon \& Corbit, 1974). According to this theory, drug addiction progresses due to the automatic modulation of drug-produced positive and negative emotional states by the central nervous system. Initially, there are high levels of positive drug effects (e.g. euphoria) and low levels of negative drug effects (e.g. withdrawal symptoms). With repeated drug use, however, the positive effects of the drug decrease and the negative effects of the drug increase, which provides the motivation for continued drug taking via negative reinforcement. Although both positive and negative reinforcement certainly play crucial roles in the development of a SUD, they do not explain all aspects of the disorder (T. E. Robinson \& Berridge, 1993).

It is now well established that drug craving and the risk of relapse can persist even after decades of abstinence, when most withdrawal symptoms have long since subsided (Hyman \& Malenka, 2001; O’Brien, Childress, Ehrman, \& Robbins, 1998; Wise, 2000; Wise \& Bozarth, 1987). Craving and relapse can be precipitated by exposure to various environmental stimuli such as drug paraphernalia as well as the people and places that have been repeatedly associated 
with the drug (Childress, Ehrman, McLellan, \& O'Brien, 1988; Childress et al., 1993, 1999; Ehrman, Robbins, Childress, \& O’Brien, 1992). These stimuli can develop conditioned reinforcing properties, capable of reinforcing behaviour in the absence of the primary drug reinforcer, despite not being intrinsically rewarding (Daniela, Gittings, \& Schenk, 2006; Schenk \& Partridge, 2001; Schuster \& Woods, 1968; S. G. Smith \& Davis, 1973; Stewart, de Wit, \& Eikelboom, 1984). Unlike the primary reinforcing effects of the drug, which undergo tolerance following repeated exposure, conditioned reinforcers can maintain their strength as a reinforcer over extended periods of time, and may even become more effective reinforcers of behaviour as a function of abstinence (Grimm, Hope, Wise, \& Shaham, 2001; P. Li et al., 2015; X. Li, Caprioli, \& Marchant, 2015).

Conditioned reinforcers can also acquire incentive motivational properties, becoming highly salient and capable of motivating approach or consummatory behaviour (Bindra, 1974, 1978; Bolles, 1972). Thus, exposure to drug-associated conditioned reinforcers can produce intense feelings of desire and craving for the drug, which can then motivate behaviour towards the acquisition and consumption of the drug they were associated with (Childress et al., 1988, 1993, 1999; Ehrman et al., 1992; Franken, Hendriks, Stam, \& Van den Brink, 2004; Garavan et al., 2000). As will be discussed in greater detail below, it has been suggested that hypersensitivity to the incentive motivational properties of drugs and drug-associated stimuli plays a major role in the development of persistent drug-craving and drug-seeking behaviour that is characteristic of SUDs (Berridge \& Robinson, 2016; T. E. Robinson \& Berridge, 1993, 2008).

\section{Studying addiction}

In order to provide more effective behavioural and pharmaceutical treatments for SUDs, it is vital to further our understanding of the mechanisms that mediate the shift from positive to negative reinforcement, the sensitisation of incentive salience attributed to drugs and drugassociated stimuli, and the inability to exert self-control over drug-taking and drug-seeking behaviour. In recent decades, much progress has been made in this regard, with substantial evidence supporting the idea that a SUD is a disorder of the brain brought on by persistent drug-induced changes in various brain mechanisms that are involved in these processes (Berridge \& Robinson, 2016; Everitt \& Robbins, 2005; Gardner, 2011; Kalivas \& Volkow, 2005; Volkow, Koob, \& McLellan, 2016; Weiss, 2005).

Neuroimaging studies in individuals that suffer from a SUD have provided valuable insight into some of the brain mechanisms underlying a SUD (Goldstein \& Volkow, 2011; 
Koob \& Volkow, 2010). Studies using human participants have several limitations, however. Firstly, researchers will often have incomplete or inaccurate information regarding drug-use history; consumption patterns, drug doses, overall intake, polydrug use, and drug purity can all vary considerably and are unlikely to be recalled accurately in the self-report questionnaires that are typically used. Secondly, varied personal histories and traits can limit the conclusions that can be drawn. For example, anxiety, depression, and high impulsivity are not only predisposing factors for drug abuse but also possible consequences of drug abuse (Parrott, 2006, 2013; Rogers et al., 2009). Finally, in an experimental setting, the administration of addictive and potentially harmful drugs to humans has obvious ethical limitations, with only low doses of some drugs being approved due to safety reasons, which limits the experimental investigation of causal relationships.

It is for these reasons that much of our current understanding of the brain mechanisms underlying compulsive drug-taking and drug-seeking behaviour has come through the use of various animal models such as self-administration, conditioned place preference (CPP), behavioural sensitisation, or drug discrimination (Carter \& Griffiths, 2009; Gardner, 2000; Koob \& Volkow, 2010; O’Brien \& Gardner, 2005; Overton, 1987; Panlilio \& Goldberg, 2007; Shaham, Shalev, Lu, De Wit, \& Stewart, 2003; Tzschentke, 2007). The use of non-human animal subjects allows for researchers to carefully control important experimental variables such as the age at which the animals are exposed to the drug, the drug dose and drug purity, as well as the duration and extent of drug exposure. Important genetic, environmental, and developmental factors can also be carefully controlled and investigated. Furthermore, a wider range of drug doses and drug types can be administered than what is ethically viable with human participants. Although no animal model can fully emulate a SUD, they do allow for the investigation of specific aspects of the disorder (Koob \& Volkow, 2010). The selfadministration paradigm is one such procedure that has proved to be particularly valuable in unravelling the behavioural and neurobiological mechanisms underlying drug-taking behaviour.

Self-administration. In the standard self-administration paradigm, laboratory animals are trained to perform a simple behavioural response (e.g. lever press, key peck, nose poke) in order to receive a drug reward. The drug is usually administered via an indwelling intravenous (i.v.) catheter (Weeks, 1962), but other methods (e.g. oral, inhalation, transdermal, intracranial) can also be used depending on the species of animal or drug being used (Mattox \& Carroll, 1996; McBride, Murphy, \& Ikemoto, 1999; Samson, Pfeffer, \& Tolliver, 1988). The 
frequency of responding maintained by drug infusions is typically compared to the responses made on a second control manipulandum (e.g. a second lever) that has no programmed consequence or against responding maintained by a vehicle/placebo infusion. A preference in responding on the manipulandum that results in the drug reward suggests that the drug is acting as a reinforcer (e.g. Corrigall \& Coen, 1989; Schenk, Colussi-Mas, Do, \& Bird, 2012; Williamson, 1997). Further evidence that the drug is acting as a reinforcer include the eventual cessation of responding (extinction) when the drug is removed or replaced with vehicle/placebo and the reinstatement of extinguished responding when the drug is subsequently reintroduced (e.g. Gerber \& Stretch, 1975; Grove \& Schuster, 1974; Pickens \& Harris, 1968). Likewise, when the consequences associated with each manipulandum (i.e. drug vs no drug) are reversed, a corresponding reversal in the preference in responding can also be observed (e.g. Goeders \& Smith, 1983; Pickens \& Harris, 1968).

Self-administration of various drugs of abuse has been reliably demonstrated in nonhuman primates (Deneau, Yanagita, \& Seevers, 1969; Gerber \& Stretch, 1975; Goldberg, Woods, \& Schuster, 1971; Lamb \& Griffiths, 1990; Thompson \& Schuster, 1964), dogs (Risner \& Jones, 1975), cats (Balster, Kilbey, \& Ellinwood, 1976), rats (Collins, Weeks, Cooper, Good, \& Russell, 1983; Weeks \& Collins, 1964), mice (Criswell, 1983), fish (Bossé \& Peterson, 2017), and even invertebrates such as crayfish (Datta, van Staaden, \& Huber, 2018) and ants (Entler, Cannon, \& Seid, 2016). Rodents (rats and mice) are the most commonly used subjects, however. With few exceptions (i.e. hallucinogens), drugs that are abused by humans are readily self-administered by laboratory animals, whereas drugs that are not abused by humans do not maintain self-administration in laboratory animals (Deneau et al., 1969; O'Connor, Chapman, Butler, \& Mead, 2011; Schuster \& Thompson, 1969). Accordingly, the self-administration procedure has proved to be an excellent method for evaluating the abuse liability of drugs and is used as a pre-clinical screening method to assess the abuse liability of novel pharmaceutical compounds (Ator \& Griffiths, 2003; Food and Drug Administration [FDA], 2010).

The pattern of drug self-administration maintained by laboratory animals is similar to that exhibited by humans who misuse drugs. For example, opiate self-administration is more constant and moderate, with little voluntary abstinence periods, while psychostimulant and alcohol self-administration is more binge-like, alternating between bursts of responding and abstinence (Deneau et al., 1969; Gardner, 2000; Harrigan \& Downs, 1978; Kramer, Fischman, \& Littlefield, 1967; Pickens \& Harris, 1968; Schenk, Gittings, Johnstone, \& Daniela, 2003; Winger \& Woods, 1973; Yokel \& Pickens, 1973). Like humans, laboratory animals are also 
capable of regulating their drug-intake. When a range of drug doses are available for selfadministration, responding changes in a compensatory manner as a function of drug dose so that drug-intake remains relatively constant (Goldberg, Hoffmeister, Schlichting, \& Wuttke, 1971; Pickens \& Harris, 1968; Weeks \& Collins, 1964). Likewise, when the work required to receive a drug injection is increased (i.e. an increase in the fixed ratio [FR] schedule), there is a proportional increase in the number of responses, within limits (Goldberg, Hoffmeister, et al., 1971; Pickens \& Harris, 1968; Schenk et al., 2012). This suggests that laboratory animals titrate their drug-intake to maintain blood/brain levels that are reinforcing, as do humans (Gardner, 2000). In order for self-administration to be more than just a model of drug-taking, however, other features of a SUD must also be evident and able to be investigated.

In most self-administration procedures, the delivery of the drug reinforcer occurs concurrently with the delivery of a discrete, neutral stimulus such as a light or tone. These stimuli are presumed to be analogous of the various environmental stimuli that become associated with drug-taking in humans (e.g. the people, places, and drug paraphernalia associated with the drug). Given repeated pairings of these stimuli with the drug over several self-administration sessions, these stimuli can become conditioned reinforcers, able to maintain responding in the absence of the primary drug reinforcer (Daniela et al., 2006; Schenk \& Partridge, 2001; Schuster \& Woods, 1968; S. G. Smith \& Davis, 1973; Stewart et al., 1984), and acquire incentive motivational properties (T. E. Robinson \& Berridge, 1993). Failure to present such stimuli can delay or even prevent the acquisition of self-administration of some drugs (Caggiula et al., 2002; Schenk \& Partridge, 2001; Schindler, Cogan, Thorndike, \& Panlilio, 2011).

To study the important role of these conditioned stimuli in maintaining drug-seeking and drug-taking behaviour, researchers have employed more complex schedules of reinforcement that are designed to model the sequence of stimuli-exposure, drug-seeking, and drug-taking, that is typical in humans (Panlilio \& Goldberg, 2007). For instance, under secondorder or chained schedules of reinforcement, responding is primarily reinforced by the presentation of these conditioned-stimuli, and only after a certain number of conditioned reinforcers have been earned, does the drug reinforcer become available (Goldberg, Kelleher, \& Morse, 1975; Schindler, Panlilio, \& Goldberg, 2002). Removal of these stimuli dramatically decreases responding, suggesting that they are acting as conditioned reinforcers and are maintaining drug-seeking behaviour until the drug itself becomes available. 
Another method developed to investigate the role of these stimuli in drug-seeking is the reinstatement paradigm (de Wit \& Stewart, 1981, 1983; Shaham et al., 2003; Stewart \& de Wit, 1987). In this procedure, following an extensive history of drug self-administration, drug reinforcement is discontinued until responding has extinguished. External stimuli are then used to provoke relapse of drug-seeking behaviour, as measured by the reinstatement of responding. Three types of stimuli have been found to reinstate responding; the presentation of conditioned stimuli/cues (Katner, Magalong, \& Weiss, 1999; McFarland \& Ettenberg, 1997; Meil \& See, 1996), re-exposure to a single dose of drug (de Wit \& Stewart, 1981, 1983; Stewart \& de Wit, 1987), and stress (Erb, Shaham, \& Stewart, 1996; Shaham, Rajabi, \& Stewart, 1996; Shaham $\&$ Stewart, 1995). Notably, these are all stimuli that are known to be prominent triggers of craving and relapse in humans, which is one of the defining characteristics of a SUD (criteria 4 of SUD).

An uncontrolled increase in drug intake is another defining characteristic of a SUD (criteria 1 of SUD), yet this is not typically observed under the limited-access (e.g. 1 hour per day) self-administration conditions most commonly used with laboratory animals. As previously mentioned, laboratory animals will maintain a relatively constant drug-intake across sessions, even when different drug-doses or reinforcement schedules are used. When daily session lengths are increased (e.g. 6 hours per day), however, a significant escalation in drug intake has been observed over successive test sessions (Ahmed \& Koob, 1998; Ahmed, Walker, \& Koob, 2000; Highgate \& Schenk, 2018; Kitamura, Wee, Specio, Koob, \& Pulvirenti, 2006; O’Dell et al., 2007; A. J. Roberts, Heyser, Cole, Griffin, \& Koob, 2000). Importantly, these extended-access self-administration conditions can also exacerbate, or are often necessary for, the development of several other features that are characteristic of SUDs (Edwards \& Koob, 2013).

For example, following unlimited access to some drugs, preferential attention was directed towards the acquisition of drugs as opposed to other naturally reinforced behaviours (criteria 7 of SUD; Bozarth \& Wise, 1985; Deneau et al., 1969). Extended self-administration access also resulted in drug-taking that was resistant to effects of punishment (e.g. foot shock; criteria 8 of SUD; Deroche-Gamonet, Belin, \& Piazza, 2004; Pelloux, Everitt, \& Dickinson, 2007; Vanderschuren \& Everitt, 2004). Furthermore, there was resistance to extinction of responding when the drug was no longer available following a history of extended access selfadministration (criteria 3 of SUD; Ahmed et al., 2000; Perry, Morgan, Anker, Dess, \& Carroll, 2006). Subjects with a history of extended access self-administration also worked significantly 
harder to obtain the drug under progressive ratio (PR) schedules, making hundreds or even thousands of responses to obtain a single dose of the drug (criteria 3 of SUD; Paterson \& Markou, 2003; Walker \& Koob, 2007; Wee, Mandyam, Lekic, \& Koob, 2008; but see Liu, Roberts \& Morgan, 2005). Moreover, both drug- and cue-induced reinstatement of extinguished drug-seeking was enhanced in animals following an escalation of intake under extended access self-administration conditions (criteria 4 of SUD; Deroche-Gamonet et al., 2004; Deroche-Gamonet, Le Moal, \& Piazza, 1999; Kippin, Fuchs, \& See, 2006; Mantsch, Yuferov, Mathieu-Kia, Ho, \& Kreek, 2004; Vanderschuren \& Everitt, 2004). It is for these reasons that long-access self-administration sessions and an escalation of drug intake have been suggested to be of particular importance for the comprehensive and valid modelling of a SUD (Ahmed, 2011; Edwards \& Koob, 2013).

The self-administration procedure has been invaluable for studying the mechanisms underlying the maintenance and reinstatement of drug-taking/seeking behaviour (Carter \& Griffiths, 2009; Gardner, 2000; Koob \& Volkow, 2010; Panlilio \& Goldberg, 2007; Shaham et al., 2003). Under the right conditions, several features of a SUD can be observed, and various manipulations can be employed to study them. Self-administration has also been useful for examining predisposing factors of drug abuse (e.g. D. Belin, Berson, Balado, Piazza, \& Deroche-Gamonet, 2011; D. Belin, Mar, Dalley, Robbins, \& Everitt, 2008; Bird \& Schenk, 2013; Horger, Shelton, \& Schenk, 1990; Lynch, 2006; Piazza, Deminière, le Moal, \& Simon, 1990; Piazza, Deminière, Le Moal, \& Simon, 1989; Schenk, Lacelle, Gorman, \& Amit, 1987; Schenk, Robinson, \& Amit, 1988). While several environmental and genetic manipulations have been shown to facilitate the acquisition of self-administration, one of the most robust effects is produced following pre-exposure to drugs under conditions that produce behavioural sensitisation.

Behavioural sensitisation. Acute administration of many drugs can produce dosedependent increases in various behaviours. Following repeated drug exposure, some drugproduced behaviours may decrease (indicating tolerance), whereas other behaviours can increase (indicating sensitisation). The latter phenomenon was first reported in the 1930s and refers to the progressive and persistent increase in drug-produced behaviour that occurs as a function of repeated exposure to some drugs (Downs \& Eddy, 1932; Seevers \& Tatum, 1931). The most commonly measured drug-produced behaviour is locomotor activity, although sensitisation of several other behaviours including sniffing, head movements, and rearing has also been reported (Pierce \& Kalivas, 1997; Post \& Rose, 1976; T. E. Robinson, 1984; Stewart 
\& Badiani, 1993). Behavioural sensitisation has been observed following repeated exposure to several different psychostimulants including cocaine, amphetamine, methylphenidate, and MDMA, as well as other classes of drugs including opioids, nicotine, and ethanol (for reviews see Kalivas \& Stewart, 1991; Pierce \& Kalivas, 1997; T. E. Robinson \& Becker, 1986; Vanderschuren \& Kalivas, 2000).

A behavioural sensitisation procedure is characterised by two phases: the development of sensitisation and the expression of sensitisation. The development of sensitisation (also called the induction or initiation of sensitisation) typically involves a regimen of repeated, intermittent, experimenter-administered drug exposure followed by a withdrawal period of at least one day. The expression of sensitisation refers to the manifestation of the sensitised behavioural response produced by subsequent re-exposure to the drug. A moderate dose of the drug is typically used so as to observe changes in behaviour without ceiling or floor effects, although full dose-effect curves are sometimes produced. A greater behavioural response in drug pretreated animals compared to vehicle/placebo pretreated controls indicates the development of a sensitised behavioural response and a leftward shift in the dose-response curve.

The acquisition of drug self-administration is also dose-dependent; self-administration of higher doses of drug is generally acquired more readily than when lower doses serve as the reinforcer (Carroll \& Lac, 1997; Schenk et al., 1993). Much like increasing the dose, pretreating animals to a regimen of drug exposure that results in behavioural sensitisation also sensitises the acquisition of self-administration (i.e. shifts the acquisition curve for self-administration to the left). For example, low doses of amphetamine and cocaine, that were usually subthreshold as a reinforcer, were reliably self-administered in rats pretreated with amphetamine (Piazza et al., 1990, 1989, 1991; Pierre \& Vezina, 1997; Vezina, Lorrain, Arnold, Austin, \& Suto, 2002) or cocaine, (Horger et al., 1990; Zhao \& Becker, 2010), respectively. For less reinforcing drugs, such as ethanol or MDMA, pretreatment can speed the acquisition of self-administration and/or increase the proportion of animals that meet an acquisition criterion (Camarini \& Hodge, 2004; Rodd-Henricks et al., 2002; van de Wetering \& Schenk, 2017). Animals pre-exposed to drugs under conditions that produce behavioural sensitisation also worked harder in order to obtain amphetamine infusions under a PR schedule (Lorrain, Arnold, \& Vezina, 2000; Mendrek, Blaha, \& Phillips, 1998; Vezina et al., 2002) and showed an enhanced escalation of cocaine intake under extended access self-administration conditions (Ferrario \& Robinson, 2007). 
These findings suggest that pre-exposure to drugs under these conditions also results in the sensitisation of the reinforcing properties of drugs. As will be discussed in the next section, the development of behavioural sensitisation involves neuroadaptations in brain mechanisms that are also involved in reward, reinforcement, and incentive motivation. For these reasons, the behavioural sensitisation procedure has been a useful tool for efficiently examining potential neuroadaptations and manipulations that are relevant to drug-taking and selfadministration, albeit without necessarily measuring drug-taking.

\section{Neurocircuitry of addiction}

In the 1950s, researchers discovered that laboratory animals would avidly perform tasks (e.g. lever pressing) in order to receive mild electrical stimulation of certain sub-cortical brain structures (Olds \& Milner, 1954). These findings were later replicated in humans (Bishop, Elder, \& Heath, 1963) and were the first of many to suggest that the activation of certain brain regions was rewarding and could positively reinforce behaviour. In the years following this discovery, the so-called 'reward circuitry' of the brain was extensively mapped using such procedures. The primary neural substrates driving reward were identified to be the dopamine (DA) neurons of the ventral tegmental area (VTA) and their efferent projections to the nucleus accumbens (NAcc) via the median forebrain bundle (Olds \& Milner, 1954). The next question for some researchers was, could improper functioning in this brain system underlie pathological responses to rewarding stimuli such as drugs?

\section{Ventral tegmental area and nucleus accumbens}

Dopamine and reinforcement. A wealth of evidence indicates that drug-produced DA activity within this VTA $\rightarrow$ NAcc mesoaccumbal system plays an essential role in the acute rewarding/reinforcing effects of drugs. Firstly, nearly all drugs of abuse have the pharmacological capacity to increase DA activity within these brain regions, either by directly facilitating the release of DA in the NAcc (in the case of psychostimulants) or by activating DAergic VTA neurons (in the case of opioids and alcohol). This was clearly demonstrated by Di Chiara and Imperato (1988) who measured extracellular DA concentrations in the NAcc using in vivo microdialysis. Drugs of abuse, such as amphetamine, cocaine, nicotine, and morphine, increased extracellular concentrations of DA by up to $1000 \%, 330 \%, 225 \%$, and $200 \%$, respectively, whereas drugs that are not abused such as imipramine (anti-depressant), atropine (anti-muscarinic drug), and diphenhydramine (antihistamine), did not increase synaptic DA concentrations (Di Chiara \& Imperato, 1988). The increase in synaptic DA produced by drugs of abuse has also been shown to directly relate to the pattern of responding 
during self-administration. Laboratory animals initially responded rapidly, which greatly increased extracellular DA levels, after which sustained responding maintained these elevated DA levels (Hurd, Weiss, Koob, And, \& Ungerstedt, 1989; Pettit \& Justice, 1991; Ranaldi, Pocock, Zereik, \& Wise, 1999; Wise et al., 1995).

Secondly, drugs of abuse are readily self-administered when intracranially administered directly into these brain regions (Bozarth, 1987; McBride et al., 1999; Routtenberg, 1972). Psychostimulants such as amphetamine are self-administered into the NAcc (Hoebel et al., 1983; A. G. Phillips, Mora, \& Rolls, 1981), while opioids such as morphine are more readily self-administered into the VTA (Bozarth \& Wise, 1981, 1982). Drugs that selectively facilitate DA neurotransmission in these brain regions are also self-administered suggesting that the DAergic action of drugs alone is reinforcing (Howell \& Byrd, 1991; Nader \& Mach, 1996; Ranaldi, Wang, \& Woolverton, 2001; Self, Belluzzi, Kossuth, \& Stein, 1996; Self \& Stein, 1992; Weed \& Woolverton, 1995; Woolverton, Goldberg, \& Ginos, 1984; Yokel \& Wise, 1978).

Thirdly, pharmacological manipulation of the DAergic system influences drug selfadministration. Administration of DA agonists decreased self-administration of various psychostimulants, including cocaine, amphetamine, and methamphetamine, producing a leftward shift in the dose-effect curve for self-administration (Barrett, Miller, Dohrmann, \& Caine, 2004; Caine \& Koob, 1994a; Munzar, Baumann, Shoaib, \& Goldberg, 1999; Yokel \& Wise, 1978). Conversely, administration of DA antagonists, either systemically or intra-NAcc, produced an increase in responding and a rightward shift in the dose-effect curve for selfadministration (Brennan, Carati, Lea, Fitzmaurice, \& Schenk, 2009; Britton et al., 1991; Caine \& Koob, 1994a; Corrigall \& Coen, 1991a; Hubner \& Edward Moreton, 1991; Koob, Le, \& Creese, 1987; G. D. Phillips, Howes, Whitelaw, Robbins, \& Everitt, 1994; G. D. Phillips, Robbins, \& Everitt, 1994; Risner \& Jones, 1976; Schenk, 2002; Yokel \& Wise, 1976). This compensatory responding produced by DA agonists and antagonists is similar to what is seen when the self-administration infusion dose is increased or decreased, respectively (Goldberg, Hoffmeister, et al., 1971; Pickens \& Harris, 1968; Weeks \& Collins, 1964).

Finally, the destruction of mesoaccumbal DA neurons decreases both the acquisition and maintenance of self-administration. Lesions of the NAcc and VTA with 6hydroxydopamine (6-OHDA) prevented the acquisition of amphetamine (Lyness, Friedle, \& Moore, 1979) and heroin (Bozarth \& Wise, 1986) self-administration, respectively. NAcc 6OHDA lesions also greatly decreased responding maintained by self-administered nicotine and 
amphetamine (Corrigall \& Coen, 1991b; Lyness et al., 1979; Singer, Wallace, \& Hall, 1982). Lesions of the both NAcc or the VTA with 6-OHDA similarly decreased the self-administration of cocaine (Caine \& Koob, 1994b; D. C. S. Roberts, Corcoran, \& Fibiger, 1977; D. C. S. Roberts, Koob, Klonoff, \& Fibiger, 1980; D. C. S. Roberts \& Koob, 1982). In these studies, the recovery of cocaine self-administration was also correlated with the level of DA depletion produced by the lesion, with rats with the greatest DA depletion failing to recover cocaine selfadministration at all (D. C. S. Roberts \& Koob, 1982; D. C. S. Roberts et al., 1980).

It is clear that the acute reinforcing effects of drugs are due to their effect on DA within the brain's 'reward' circuitry. Indeed, with few exceptions, this is the only pharmacological effect common to all drugs of abuse. Of course, the brain's 'reward' circuitry did not evolve to engender drug-taking behaviour, but rather to reinforce biologically essential behaviours such as feeding, drinking, sexual behaviour, maternal/paternal behaviour, and social interaction, all of which induce DAergic activity in these brain regions (Bassareo \& Di Chiara, 1999a; Hansen, Bergvall, \& Nyiredi, 1993; Manuel Mas, Fumero, \& González-Mora, 1995; Pfaus et al., 1990; White \& Milner, 1992). Drugs of abuse are capable of activating these brain regions to a far greater extent than natural rewards, however, and therefore can serve as incredibly powerful reinforcers that can direct behaviour towards the acquisition and consumption of drugs at the expense of other naturally reinforced behaviours.

Dopamine and incentive motivation. Given the vital role of mesoaccumbal DAergic mechanisms in the reinforcing effects of drugs and other rewards, it was initially presumed that DA mediated the subjective experience of pleasure (Olds, 1956; Wise, 1980, 1982). Early studies conducted by Robinson and Berridge suggested otherwise, however. DAergic manipulations had no effect on specific measures of pleasure but did have a significant impact on the motivation to obtain and consume rewards (Berridge \& Valenstein, 1991; Berridge, Venier, \& Robinson, 1989). This led to their suggestion that positive reinforcers/rewards are both 'liked' (referring to the subjective pleasurable effects of the drug) as well as 'wanted' (referring to the incentive motivational properties of the drug/drug-associated stimuli). Although both drug 'liking' and 'wanting' can influence how reinforcing a drug is, they argued that mesolimbic DA activity primarily serves to promote the latter (T. E. Robinson \& Berridge, 1993).

Additional support for this idea has grown over the years (for recent reviews see Berridge \& Kringelbach, 2015; Berridge \& Robinson, 2016). For example, NAcc DA release was increased by the consummation of a food-reward but also by the anticipation of food 
reward, which was potentiated by food-deprivation (Bassareo \& Di Chiara, 1999b). NAcc DA release or DA neuron firing was also greatest when rewards were novel and when they were presented unexpectedly (Baldo \& Kelley, 2007; Bassareo, De Luca, \& Di Chiara, 2002; Bassareo \& Di Chiara, 1997, 1999a; Schultz, Dayan, \& Montague, 1997). These findings suggested a role of DA in incentive and learning, not just pleasure. Studies in human drug users have shown similar findings. Suppression of DA neurotransmission had no effect of subjective ratings of drug-produced euphoria but did significantly decrease the desire to consume drugs (Brauer \& de Wit, 1997; Leyton, Casey, Delaney, Kolivakis, \& Benkelfat, 2005). Further, increases in brain DA activity, as measured by neuroimaging, were more correlated with subjective ratings of drug or food 'wanting' than with pleasure ratings (C. T. Smith, Dang, Cowan, Kessler, \& Zald, 2016; Volkow et al., 2002).

An important role of DAergic mechanisms in conditioned reinforcement has also been demonstrated, further evidencing the role of DA in 'wanting'. For example, presentation of food or drug-associated stimuli increased the firing rate of VTA neurons and enhanced DA release in the NAcc core (Ito, Dalley, Howes, Robbins, \& Everitt, 2000; P. E. M. Phillips, Stuber, Heien, Wightman, \& Carelli, 2003; Schultz, 1998). Acquisition of conditioned approach behaviour towards reward-associated stimuli was also prevented by lesions of the NAcc core or the administration of DA antagonists (Di Ciano, Cardinal, Cowell, Little, \& Everitt, 2001; Parkinson, Olmstead, Burns, Robbins, \& Everitt, 1999). Conditioned reinforcement was similarly decreased by these DAergic manipulations (Hall, Parkinson, Connor, Dickinson, \& Everitt, 2001; Parkinson et al., 1999). Further, drug-seeking induced by exposure to conditioned stimuli previously associated with drug-taking was either attenuated or potentiated by systemic or intra-accumbal administration of DA antagonists or agonists, respectively (Cervo, Carnovali, Stark, \& Mennini, 2003; Crombag, Grimm, \& Shaham, 2002; Di Ciano, Underwood, Hagan, \& Everitt, 2003; Gál \& Gyertyán, 2006; Gilbert et al., 2005; Pilla et al., 1999; Schenk, Gittings, \& Colussi-Mas, 2011)

It is now, therefore, widely accepted that the primary role of mesoaccumbal DA is not to mediate reward pleasure or 'liking', but to imbue rewards and associated stimuli with incentive salience, making them 'wanted', and motivating-goal directed behaviour towards their acquisition (Berridge \& Robinson, 2016; Everitt \& Robbins, 2005; Kalivas \& Volkow, 2005; Koob \& Volkow, 2010; Wise, 2008). According to Robinson and Berridge's popular incentive sensitisation theory of addiction, repeated drug exposure results in the sensitisation of this neural system and, therefore, the sensitisation of the incentive motivational properties 
of drugs and drug-associated stimuli (Berridge \& Robinson, 2016; T. E. Robinson \& Berridge, 1993, 2000, 2001, 2008).

A large source of evidence in support of this theory comes from studies that have used the behavioural sensitisation procedure. The locomotor activating effects of drugs have long been known to also be mediated by central DAergic mechanisms (Carlsson, Lindqvist, Magnusson, \& Waldeck, 1958; Costall \& Naylor, 1979; Hornykiewicz, 1966; Ungerstedt, 1979). Extracellular concentrations of DA are positively correlated with drug-produced locomotor activity (Hurd et al., 1989; Kalivas \& Duffy, 1990; Sharp, Zetterström, Ljungberg, \& Ungerstedt, 1987; Steinpreis \& Salamone, 1993) and manipulations that attenuate DAergic activity decrease locomotor activity (Garrett \& Holtzman, 1994; Pijnenburg, Honig, \& Van Rossum, 1975; Schindler \& Carmona, 2002). It is not surprising, then, that behavioural sensitisation is accompanied by sensitised DAergic mechanisms (Kalivas \& Stewart, 1991; Pierce \& Kalivas, 1997; Vanderschuren \& Kalivas, 2000). For example, repeated drug exposure under conditions that produced behavioural sensitisation also enhanced drugproduced NAcc DA release (Kalivas \& Duffy, 1990; Kalivas, Duffy, \& White, 1998; Parsons \& Justice, 1993; Pettit, Pan, Parsons, \& Justice, 1990; T. E. Robinson, Jurson, Bennett, \& Bentgen, 1988), increased dendritic spine density in NAcc medium spiny neurons (MSN; Ball, Wellman, Fortenberry, \& Rebec, 2009; Brown \& Kolb, 2001; Yilin Li, Acerbo, \& Robinson, 2004; Yilin Li, Kolb, \& Robinson, 2003), and resulted in an upregulation of DA D 1 receptors (Henry \& White, 1991; Yong Li et al., 1999; Wolf, White, \& Hu, 1994).

The VTA has been demonstrated to be a crucial substrate involved in the development of behavioural sensitisation (Vanderschuren \& Kalivas, 2000). Repeated intra-VTA, but not intra-NAcc, administration of various drugs including amphetamine, cocaine, or morphine, enhanced the locomotor activating effect produced by subsequent systemic or intra-NAcc drug exposure (Cador, Bjijou, \& Stinus, 1995; Cornish \& Kalivas, 2001; Hooks, Jones, Liem, \& Justice, 1992; Kalivas \& Duffy, 1987; Kalivas \& Weber, 1988; Vezina, Kalivas, \& Stewart, 1987). In contrast, NAcc activation appears to be crucial for the expression of behavioural sensitisation (Vanderschuren \& Kalivas, 2000). Following repeated systemic or intra-VTA exposure of various drugs, intra-NAcc, but not intra-VTA, administration of psychostimulants or selective dopamine agonists resulted in the expression of sensitised locomotor activity (Abrahao, Quadros, \& Souza-Formigoni, 2011; Cador et al., 1995; Cunningham, Finn, \& Kelley, 1997; Paulson \& Robinson, 1991; Perugini \& Vezina, 1994; Pierce \& Kalivas, 1995; Vanderschuren, Schoffelmeer, Mulder, \& De Vries, 1999; Vezina, 1996). 
As previously mentioned, repeated exposure to drugs under conditions that produce behavioural sensitisation has been shown to also sensitise the reinforcing/incentive properties of drugs, as indicated by the facilitated acquisition of drug self-administration, higher breakpoints under PR schedules, and the more rapid escalation of drug-intake under extended-access conditions (Camarini \& Hodge, 2004; Ferrario \& Robinson, 2007; Horger et al., 1990; Lorrain et al., 2000; Mendrek et al., 1998; Piazza et al., 1990, 1989, 1991; Pierre \& Vezina, 1997; Rodd-Henricks et al., 2002; van de Wetering \& Schenk, 2017; Vezina et al., 2002; Zhao \& Becker, 2010; but see Horger, Giles, \& Schenk, 1992; Schenk, Snow, \& Horger, 1991). Behavioural sensitisation has also been shown to enhance the reinforcing effects of drugs as determined by the CPP paradigm (Gaiardi et al., 1991; Lett, 1989; Shippenberg \& Heidbreder, 1995). Evidence suggests that the development of sensitisation to both the locomotor-activating and reinforcing effects of drugs involves overlapping DAergic mechanisms (for reviews see Berridge \& Robinson, 2016; Pierce \& Kalivas, 1997; T. E. Robinson \& Berridge, 1993, 2008; Vanderschuren \& Pierce, 2010; Vezina, 2004). For example, manipulations that prevent the development of behavioural sensitisation also prevent the subsequent facilitation of drugtaking; treatment with a $\mathrm{DA} \mathrm{D}_{1}$ receptor antagonist blocked both the development of amphetamine locomotor sensitisation (Vezina, 1996; Vezina \& Stewart, 1989) and the sensitised acquisition of amphetamine self-administration (Pierre \& Vezina, 1998). These findings suggest that that the sensitised mesoaccumbal DAergic mechanisms that underlie behavioural sensitisation also underlie the sensitisation of the reinforcing/incentive motivational properties of drugs.

The reinforcing/incentive motivational properties of conditioned stimuli similarly appear to be sensitised following regimens of repeated drug exposure that result in behavioural sensitisation. For example, a sensitising pretreatment regimen of amphetamine or cocaine exposure facilitated the acquisition of conditioned approach behaviour towards stimuli previously associated with reward (Harmer \& Phillips, 1998; Taylor \& Jentsch, 2001) and enhanced responding maintained by conditioned reinforcers (Cunningham \& Kelley, 1992; Di Ciano, 2008; Mead, Crombag, \& Rocha, 2004; Taylor \& Horger, 1999; Wyvell \& Berridge, 2001). Specific firing patterns of DA neurons produced by exposure to drug-conditioned stimuli were also potentiated following a sensitising regimen of amphetamine exposure (Tindell, Berridge, Zhang, Peciña, \& Aldridge, 2005).

Although there is much evidence to suggest that repeated, intermittent exposure to drugs of abuse results in the sensitisation of the mesoaccumbal DA system and the incentive 
motivational properties of drugs/drug-associated stimuli, sensitisation is not always a result of repeated drug exposure. If drug administration occurs relatively constantly, rather than intermittently, tolerance to both the locomotor and DA releasing effects of drugs can develop instead, especially if examined under shorter withdrawal periods (Pierce \& Kalivas, 1997; Vanderschuren \& Kalivas, 2000). The expression of sensitisation is also heavily contextdependent, often requiring the same environmental and contextual stimuli where the drugs were originally administered (e.g. Ball, Budreau, \& Rebec, 2006; Stewart \& Vezina, 1987; Vezina \& Stewart, 1984). This has been suggested to explain the mixed findings from studies examining sensitisation in humans (T. E. Robinson \& Berridge, 2008; Vezina \& Leyton, 2009). While an extensive review of the incentive sensitisation theory of addiction is outside the scope of the present thesis, the important role of central DAergic mechanisms and incentive motivation in addiction is undisputed. It is also clear, however, that several other brain regions, circuits, neurotransmitters systems, and mechanisms also play crucial roles.

\section{Amygdala}

Like the NAcc, the basolateral amygdala (BLA) receives DAergic input from the VTA, while glutamatergic output neurons of the BLA innervate the NAcc as well as the prefrontal cortex (PFC). Acute drug administration has been shown to significantly increase extracellular DA in the BLA (Hurd, McGrego, \& Pontén, 1997; Young \& Rees, 1998), as has presentations of conditioned stimuli previously associated with reward (Harmer \& Phillips, 1999; Hori, Tanaka, \& Nomura, 1993; Nomura, Izaki, Takita, Tanaka, \& Hori, 2004; Weiss et al., 2000). The BLA has also been shown to modulate the increases in NAcc DA produced by drug exposure or conditioned stimuli (Hurd et al., 1997; Jones et al., 2010; Louilot, Simon, Taghzouti, \& Le Moal, 1985).

An important role of the BLA in mediating the effects of stimuli previously associated with drug reward has been demonstrated. Excitotoxic lesions, or inactivation of the BLA with lidocaine, severely impaired the acquisition and maintenance of drug-seeking under secondorder schedules of reinforcement (Alderson, Robbins, \& Everitt, 2000; Kantak, Black, Valencia, Green-Jordan, \& Eichenbaum, 2002; Whitelaw, Markou, Robbins, \& Everitt, 1996). Bi-lateral infusion of a DA antagonist into the BLA similarly attenuated drug-seeking under a second-order schedule, while pharmacological disconnection of the glutamatergic connections from the BLA to the NAcc had the same effect (Di Ciano \& Everitt, 2004). Lesions of the BLA, local inactivation with tetrodotoxin/lidocaine, or bi-lateral infusions of DA antagonists, also attenuated the reinstatement of drug-seeking behaviour produced by the presentation of 
conditioned cues (Fuchs \& See, 2002; Grimm \& See, 2000; Kantak et al., 2002; McLaughlin \& See, 2003; Meil \& See, 1997; See, Kruzich, \& Grimm, 2001). Interestingly, such manipulations had no effect on the acquisition or maintenance of self-administration or on the reinstatement of drug-seeking produced by a drug prime or stress, suggesting that the BLA may play a selective role in mediating the effects of conditioned-stimuli (Grimm \& See, 2000; McFarland, Davidge, Lapish, \& Kalivas, 2004; McFarland \& Kalivas, 2001; Meil \& See, 1997; See, Fuchs, Ledford, \& Mclaughlin, 2006; Whitelaw et al., 1996).

The central nucleus of the amygdala (CeA), in contrast, appears to play an important role in the acute, primary reinforcing effects of various drugs of abuse since lesions of the $\mathrm{CeA}$, or local administration of various antagonists, decreased self-administration of cocaine, opioids, and alcohol (Caine, Heinrichs, Coffin, \& Koob, 1995; Dyr \& Kostowski, 1995; Heyser, Roberts, Schulteis, \& Koob, 1999; Hyytiä \& Koob, 1995; McGregor \& Roberts, 1993; Möller, Wiklund, Sommer, Thorsell, \& Heilig, 1997). The CeA also plays a major role in stress responses. Stress-induced reinstatement of drug-seeking requires the activation of norepinephrine (NE; noradrenalin), and the stress hormone, corticotrophin-releasing factor (CRF), in the CeA (see Shaham et al., 2003). Acute withdrawal from drugs also potentiates anxiety-like responses to acute stressors, which has similarly been shown to require CRF activity within the CeA (Koob, 2008). Neuroadaptations within the CeA and the CRF system following repeated drug exposure have been implicated in the development of long-term negative emotional states that have been suggested play a major role in negatively reinforcing drug-seeking and drug-taking (Koob et al., 2014; Koob \& Le Moal, 2008a; Piazza \& Le Moal, 1996).

\section{Dorsal striatum}

The dorsal striatum, which contains the caudate nucleus and putamen (C-P), has been identified as a key brain region involved in habitual, stimulus-driven behaviour that has been suggested to contribute to the maintenance and relapse of drug-taking (Everitt et al., 2008; Everitt \& Robbins, 2005, 2016). Reinforcement learning, such as during self-administration, is thought to involve two processes: one associating the behaviour with the consequence (actionoutcome), and one associating the stimuli with the behaviour (stimulus-response). These processes govern goal-directed and stimulus-driven behaviour, respectively (Balleine \& O'Doherty, 2010). Initially, behaviours are goal-directed and performed with conscious regard for the outcome, but with extensive repetition, behaviour can become reflexive and habitual, driven primarily by the presentation of conditioned-stimuli (Adams, 1982; Tricomi, Balleine, 
\& O'Doherty, 2009). Such habitual behaviour is resistant to extinction and can persist even when the outcome/reward has been significantly devalued (Adams, 1982; Tricomi et al., 2009).

Considerable evidence suggests that drug-seeking behaviour can become habitual and stimulus-driven following repeated drug use (Everitt et al., 2008; Everitt \& Robbins, 2005, 2016). For example, cocaine, nicotine, or ethanol-seeking on chained-schedules of reinforcement or under extinction conditions was decreased by reward devaluation when tested following a short history of self-administration, which suggests that drug-seeking under these conditions was goal-directed (Clemens, Castino, Cornish, Goodchild, \& Holmes, 2014; Corbit, Nie, \& Janak, 2012; Olmstead, Lafond, Everitt, \& Dickinson, 2001; Zapata, Minney, \& Shippenberg, 2010). Following a more extensive history of self-administration, however, drugseeking was no longer affected by reward devaluation procedures, suggesting that drug-seeking had become habitual (Clemens et al., 2014; Corbit et al., 2012; Dickinson, Wood, \& Smith, 2002; Miles, Everitt, \& Dickinson, 2003; Olmstead et al., 2001; Zapata et al., 2010).

The dorsolateral striatum (DLS) in particular has been heavily implicated in stimulusdriven behaviour, whereas the dorsomedial striatum (DMS), like the ventral striatum (i.e. NAcc), appears to be more involved in goal-directed behaviour (Balleine \& O'Doherty, 2010; Shiflett, Brown, \& Balleine, 2010; Yin, Knowlton, \& Balleine, 2004; Yin, Ostlund, Knowlton, $\&$ Balleine, 2005). This is consistent with differences in the anatomical connectivity of these regions; the DMS receives excitatory input primarily from the medial PFC, while the DLS receives more excitatory input from sensorimotor cortices (Crittenden \& Graybiel, 2011; Voorn, Vanderschuren, Groenewegen, Robbins, \& Pennartz, 2004). It has, therefore, been suggested that the developmental of habitual drug-seeking behaviour reflects the increasing involvement of the dorsal striatum, and in particular, the DLS (Everitt et al., 2008; Everitt \& Robbins, 2005, 2016).

Accumulating evidence supports this idea. Well-established drug-seeking behaviour maintained by conditioned stimuli on a second-order schedule of reinforcement was accompanied by enhanced DA release in the dorsal, but not the ventral striatum (Ito, Dalley, Robbins, \& Everitt, 2002), and was decreased by intra-dorsal striatal administration of DA or glutamate antagonists (Vanderschuren, Di Ciano, \& Everitt, 2005). Inactivation of the DLS in particular, but not the DMS, decreased well-established drug-seeking maintained by conditioned stimuli whereas the reverse was found when drug-seeking was newly acquired (Murray, Belin, \& Everitt, 2012). Similarly, well-established drug-seeking behaviour produced by conditioned stimuli that was shown to be insensitive to reward devaluation (i.e. habitual) 
was decreased by intra-DLS administration, but not by intra-DMS administration, of DA antagonists (Corbit et al., 2012; Zapata et al., 2010). In contrast, newly-acquired drug-seeking behaviour that was sensitive to reward devaluation (i.e. goal-directed) was not decreased by intra-DLS administration but was decreased by intra-DMS administration of DA antagonists (Corbit et al., 2012; Zapata et al., 2010).

Habitual behaviour provides an efficient means of performing repetitive behaviour with little top-down, cognitive processing (Canales, 2005; Jog, Kubota, Connolly, Hillegaart, \& Graybiel, 1999). However, habitual behaviour needs to be amenable by top-down cognitive process in case the habitual behaviour becomes maladaptive. Evidence suggests that habitual drug-taking/seeking behaviour is often associated with a deficit in prefrontal cortical top-down cognitive processes, which prevents adequate control over the maladaptive and habitual drugseeking behaviour (Dalley, Everitt, \& Robbins, 2011; Jentsch \& Taylor, 1999; Robbins \& Everitt, 1999).

\section{Prefrontal cortex}

The PFC receives DAergic afferents from the VTA, while excitatory glutamatergic efferents of the PFC innervate the NAcc, VTA, and the dorsal striatum. Thus, the positioning of the PFC allows for the direct regulation of important DAergic regions involved in incentive salience as well as both goal-directed and stimulus-driven behaviour. Neuroimaging studies in humans with a SUD have shown that drug administration or the presentation of drug-associated stimuli increases PFC activity, and this is correlated with subjective measures of drug-craving (for reviews see Goldstein \& Volkow, 2011; Koob \& Volkow, 2016). In rodents, anatomical and functional analogues of important human PFC brain regions include the orbitofrontal cortex (OFC) and the medial PFC, which contains the anterior cingulate, prelimbic, and infralimbic cortices (Dalley, Cardinal, \& Robbins, 2004; Koob \& Volkow, 2016; Uylings, Groenewegen, \& Kolb, 2003). These brain regions are similarly activated by drug administration, or the presentation of drug-associated stimuli and evidence suggests that these brain regions play an important role in mediating drug-seeking behaviour (Koob \& Volkow, 2010, 2016; Shaham et al., 2003; Weiss, 2005).

Inactivation of the medial PFC (anterior cingulate or prelimbic cortex) or the OFC attenuated the reinstatement of previously extinguished drug-seeking behaviour produced by acute drug exposure, conditioned-stimuli, or stress (Capriles, Rodaros, Sorge, \& Stewart, 2003; Fuchs, Evans, Parker, \& See, 2004; McLaughlin \& See, 2003; Park et al., 2002). DAergic activity in the PFC appears to be particularly important since local infusions of various DA 
antagonists into these brain regions similarly attenuated drug-, cue-, or stress-induced reinstatement (Capriles et al., 2003; McFarland \& Kalivas, 2001; Park et al., 2002). Moreover, local infusions of DA or cocaine into the medial PFC was sufficient to reinstate drug-seeking (Park et al., 2002). An important role of glutamatergic outputs from the PFC to the NAcc in mediating drug-seeking behaviour has also been demonstrated since drug-seeking reinstated by intra-medial PFC cocaine was blocked by local administration of glutamate antagonists into the NAcc (Park et al., 2002). Pharmacological disconnection of glutamate efferents from the PFC to the NAcc also blocked cocaine-induced reinstatement (McFarland \& Kalivas, 2001). Further, cocaine-induced reinstatement was associated with PFC-mediated increases in NAcc glutamate (McFarland, Lapish, \& Kalivas, 2003).

Although widespread PFC activity is associated with craving and drug-seeking produced by drugs or drug-associated stimuli, widespread hypoactivity of the PFC is apparent during times of withdrawal or during tasks that involve higher-order cognitive and emotional control (Goldstein \& Volkow, 2011; Koob \& Volkow, 2010, 2016). Humans with a SUD show impaired performance on PFC-dependant tasks involving attention, cognitive flexibility, reward valuation, inhibitory control, and decision making (Goldstein \& Volkow, 2011; Koob $\&$ Volkow, 2010, 2016). These deficits have been associated with increased drug use, greater likelihood of relapse, and overall worse treatment outcomes (Aharonovich et al., 2006). Parallel studies in laboratory animals have shown similar results. Impairments in reversal learning (an OFC-dependant task) were observed following repeated cocaine exposure in both rats and monkeys (Calu et al., 2007; Jentsch, Olausson, de la Garza, \& Taylor, 2002; Schoenbaum, Saddoris, Ramus, Shaham, \& Setlow, 2004). Impairments have also been observed in working memory and sustained attention tasks (PFC-dependant tasks) following self-administration of cocaine under-extended access, but not limited-access conditions (Briand, Flagel, et al., 2008; Briand, Gross, \& Robinson, 2008; George, Mandyam, Wee, \& Koob, 2008). In one of these studies, these deficits were also associated with decreased $\mathrm{DA}_{2}$ receptor messenger RNA (mRNA) in the medial PFC and OFC (Briand, Flagel, et al., 2008), a finding that is consistent with those from human neuroimaging studies (see Goldstein \& Volkow, 2011; Koob \& Volkow, 2010, 2016).

It should be stated that the review on the neurocircuitry/neurobiology of addiction given here is by no means exhaustive, which would be outside of the scope of the present thesis. The brain regions and mechanisms covered here are arguably some of those that are most relevant to addiction, but also to the current thesis. For more in-depth reviews that include other relevant 
brain regions and mechanisms see (Berridge \& Robinson, 2016; Gardner, 2011; Goldstein \& Volkow, 2011; Kalivas \& Volkow, 2005; Koob \& Volkow, 2010, 2016; Shaham et al., 2003; Vanderschuren \& Pierce, 2010; Weiss, 2005).

\section{MDMA}

The behavioural and neurobiological effects of repeated exposure to most drugs of abuse such as cocaine, amphetamine, opioids, and alcohol, have been relatively well studied. The effects of repeated exposure to \pm 3 ,4-methylenedioxymethamphetamine (MDMA), is less understood, however.

\section{MDMA/ecstasy use disorders}

MDMA (commonly referred to as 'ecstasy' or 'molly') is a popular recreational drug of abuse, particularly in New Zealand and Australia. Because MDMA is often adulterated with a variety of other compounds, the term ecstasy will be used throughout this thesis when outside of controlled clinical or pre-clinical settings. It is estimated that there are approximately 21 million ecstasy users worldwide (United Nations Office on Drugs and Crime [UNODC], 2019). For comparison, there are approximately 18 million cocaine users and 188 million cannabis users (UNODC, 2019). As is the case with other drugs of abuse, a proportion of ecstasy users develop symptoms consistent with a SUD. For example, some users reported: taking ecstasy in larger amounts or for longer periods than intended (Cottler, Womack, Compton, \& BenAbdallah, 2001); wanting to cut down or stop using ecstasy but not managing to (Topp, Hall, \& Hando, 1997); cravings and urges to use ecstasy (Hopper et al., 2006); continued usage, despite knowing they had a physical or psychological problem that could have been caused or exacerbated by ecstasy (Cottler, Leung, \& Abdallah, 2009; Yen \& Hsu, 2007); tolerance to the positive effects of ecstasy (Parrott, 2005; Peroutka, Newman, \& Harris, 1988); and the development of withdrawal symptoms (Cottler et al., 2001; McKetin et al., 2014).

Ecstasy abuse has contributed to a number of deaths due to MDMA-induced hyperthermia, renal failure, hepatic toxicity, cardiac arrhythmia, rhabdomyolysis, and disseminated intravascular coagulation (Chadwick, Curry, Linsley, Freemont, \& Doran, 1991; Dykhuizen, Brunt, Atkinson, Simpson, \& Smith, 1995; Fineschi \& Masti, 1996; Freedman, Johanson, \& Tancer, 2005; García-Repetto et al., 2003; Khakoo, Coles, Armstrong, \& Barry, 1995; Schifano et al., 2003; Screaton et al., 1992). The long-term consequences associated with the repeated use of MDMA may be greater than the risk of death from acute toxicity, however. Ecstasy abuse has been attributed to the onset of several severe psychopathologies including depression, anxiety, panic attacks, paranoid psychosis, mixed affective psychosis, and 
hallucinations (Cassidy \& Ballard, 1994; McGuire, Cope, \& Fahy, 1994; Parrott, Sisk, \& Turner, 2000; Series, Boeles, Dorkins, \& Peveler, 1994; Topp, Hando, Dillon, Roche, \& Solowij, 1999; Williamson, 1997; Yen \& Hsu, 2007). Ecstasy abuse has also been associated with cognitive deficits in memory and decision-making (Halpern et al., 2004; Parrott, Lees, Garnham, Jones, \& Wesnes, 1998; Roiser, Rogers, Cook, \& Sahakian, 2006). As will be described further below, repeated ecstasy/MDMA use has also been associated with severe neurodegeneration in humans as well as laboratory animals, which might underlie some of the aforementioned cognitive and psychological consequences of ecstasy abuse. Given the popularity of MDMA/ecstasy as a drug of abuse, and the recent developments for the use of MDMA as an adjunct for the psychotherapy of post-traumatic stress disorder, there is a clear need for a better scientific understanding of the drug and the effect of repeated exposure on brain and behaviour.

\section{MDMA self-administration}

The reinforcing effects of MDMA have been less extensively studied than the reinforcing effects of other drugs of abuse. Nonetheless, like other drugs of abuse, MDMA is reliably self-administered by various laboratory animals (Aarde, Miller, Creehan, Vandewater, \& Taffe, 2015; Ball et al., 2015; Ball, Walsh, \& Rebec, 2007; Ball \& Slane, 2012, 2014; Beardsley, Balster, \& Harris, 1986; Bird \& Schenk, 2013; Bradbury et al., 2013; Colussi-Mas, Wise, Howard, \& Schenk, 2010; Creehan, Vandewater, \& Taffe, 2015; Do \& Schenk, 2013; Feduccia, Kongovi, \& Duvauchelle, 2010; Gould et al., 2011; Oakly, Brox, Schenk, \& Ellenbroek, 2013; Reveron, Maier, \& Duvauchelle, 2010; Schenk \& Bradbury, 2015; Schenk et al., 2007; Z. Wang \& Woolverton, 2007). Once acquired, MDMA self-administration is comparable to the self-administration of other psychostimulant drugs of abuse such as cocaine or amphetamine. For example, the dose-effect curve for MDMA self-administration was in the shape of an inverted U (Daniela, Brennan, Gittings, Hely, \& Schenk, 2004; Ratzenboeck, Saria, Kriechbaum, \& Zernig, 2001; Schenk et al., 2003). Halving the MDMA infusion dose resulted in the doubling of the number of responses so that drug intake $(\mathrm{mg} / \mathrm{kg})$ remained relatively constant (Do \& Schenk, 2013; Reveron et al., 2010; Schenk et al., 2012). Likewise, increasing in the FR response requirement resulted in the proportional increase in lever pressing (Daniela et al., 2006; Schenk et al., 2011). Replacing MDMA with its vehicle solution resulted in the extinction of the operant behaviour, which was subsequently reinstated by the reintroduction of MDMA (Daniela et al., 2006; Schenk et al., 2011). Further, extinguished drug-seeking behaviour following MDMA self-administration was reinstated by priming injections of 
MDMA, cocaine, or yohimbine (stress inducer), or by the presentation of a light stimulus that was previously paired with MDMA infusions (Ball et al., 2015, 2007; Schenk et al., 2011; Schenk, Hely, Gittings, Lake, \& Daniela, 2008). Like other drugs of abuse, stimuli that are paired with MDMA infusions can also develop conditioned reinforcing properties and play an important role in the maintenance of MDMA self-administration (Daniela et al., 2006).

The acquisition of MDMA self-administration, however, exhibits a profile that differs from that of other psychostimulants. Firstly, the acquisition of MDMA self-administration proceeds with a protracted-time course. Response rates for MDMA are initially low, and acquisition of reliable MDMA self-administration ( $1 \mathrm{mg} / \mathrm{kg} /$ infusion) typically requires around 15, 2-hour daily sessions (Schenk et al., 2012). In contrast, the self-administration of cocaine, amphetamine, or methamphetamine is usually reliably acquired within a few days (Carroll \& Lac, 1997; Highgate \& Schenk, 2018; Maan, Highgate, \& Schenk, 2020; Schenk et al., 2007). Secondly, only about $50 \%$ of rats meet acquisition criteria for reliable MDMA selfadministration under these conditions (Schenk et al., 2012) whereas virtually all animals acquire cocaine, amphetamine, or methamphetamine self-administration (Carroll \& Lac, 1997; Do \& Schenk, 2013; Highgate \& Schenk, 2018; Maan et al., 2020). These differences might reflect the unique pharmacology of MDMA compared to other psychostimulants.

\section{Pharmacology of MDMA}

MDMA is a ring-substituted phenethylamine and is structurally similar to methamphetamine (psychostimulant) and mescaline (hallucinogen). Unless otherwise stated, all mention of MDMA in this thesis will refer to the racemic mixture (i.e. \pm MDMA), which contains equal amounts of both S-(+)-MDMA and R-(-)-MDMA.

Pharmacokinetics. MDMA is primarily metabolised in the liver by various cytochrome P450 enzymes and catechol- $O$-methyltransferase (COMT) via two main pathways (Figure 1.1; de la Torre \& Farré, 2004; de la Torre et al., 2004; Maurer, Bickeboeller-Friedrich, Kraemer, $\&$ Peters, 2000). In the first pathway, MDMA is $O$-demethylenated by P450 enzymes into 3,4dihydroxymethamphetamine (HHMA), which is then $O$-methylated by COMT into 4-hydroxy3-methoxymethamphetamine (HMMA). In the second pathway, MDMA is first $\mathrm{N}$ demethylated by P450 enzymes into 3,4-methylenedioxyamphetamine (MDA), which is then $O$-demethylenated by $\mathrm{P} 450$ enzymes and subsequently $O$-methylated by COMT to form to 3,4dihydroxyamphetamine (HHA) and 4-hydroxy-3-methoxyamphetamine (HMA), respectively. The half-life of $\sim 1-2 \mathrm{mg} / \mathrm{kg}$ of orally administered MDMA is approximately 7 hours in humans (de la Torre et al., 2000; Kolbrich et al., 2008; M. Mas et al., 1999) and 1 hour in rats (Baumann 
et al., 2009). Higher doses have been shown to significantly increase the time for MDMA to be eliminated, however, indicating that the metabolising enzymes are saturable (Baumann et al., 2009; de la Torre et al., 2000). The metabolites of MDMA have much longer half-lives, and some of which are also bio-active (Baumann et al., 2009; de la Torre \& Farré, 2004; de la Torre et al., 2004). MDA, in particular, has potent effects on the monoamine system, much like MDMA (Stone, Stahl, Hanson, \& Gibb, 1986).

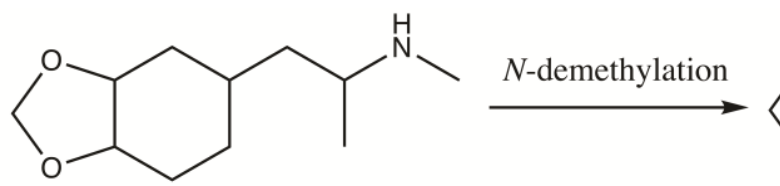

MDMA<smiles>CNC(C)CC1CCC(O)C(O)C1</smiles>

HHMA<smiles>CNC(C)CC1CCC(O)C(OC)C1</smiles>

HMMA<smiles>CC(N)CC1CCC2OCOC2C1</smiles>

MDA<smiles>CC(C)OC1CCC(O)C(O)C1</smiles>

HHA $O$-methylation<smiles>COC1CC(CC(C)N)CCC1O</smiles>

HMA

Figure 1.1. Primary metabolic pathways of MDMA. MDMA: 3,4methylenedioxymethamphetamine; HHMA: 3,4-dihydroxymethamphetamine; HMMA: 4hydroxy-3-methoxymethamphetamine; MDA: 3,4-methylenedioxyamphetamine; HHA: 3,4dihydroxyamphetamine; HMA: 4-hydroxy-3-methoxyamphetamine. 
Pharmacodynamics. MDMA is a potent releasing agent/reuptake inhibitor of the monoamine neurotransmitters: serotonin (5-HT), DA, and NE. This is accomplished via multiple, synergistic mechanisms. Acting as a substrate for the monoamine transporters, MDMA is able to enter the neuron where it inhibits/reverses vesicular monoamine transporter 2 (VMAT), which results in substantially increased concentrations of monoamines in the cytosol (Bogen, Haug, Myhre, \& Fonnum, 2003; Eiden \& Weihe, 2011; Fitzgerald \& Reid, 1990; Fleckenstein et al., 2002; Gu \& Azmitia, 1993; Rudnick \& Wall, 1992; Sabol \& Seiden, 1998; Schuldiner, Steiner-Mordoch, Yelin, Wall, \& Rudnick, 1993; Sulzer \& Rayport, 1990). MDMA also inhibits/reverses the monoamine transporters, possibly via agonistic activity at trace amine-associated receptor 1 (TAAR1; Berry, Gainetdinov, Hoener, \& Shahid, 2017; Bunzow et al., 2001; Miller, 2011), which results in the substantial efflux of the already increased cytosolic concentrations of monoamines into the synapse while also preventing their reuptake (Battaglia, Brooks, Kulsakdinun, \& De Souza, 1988; Berger, Gu, \& Azmitia, 1992; Cleary \& Docherty, 2003; Cole \& Sumnall, 2003; Crespi, Mennini, \& Gobbi, 1997; Fitzgerald \& Reid, 1990; Gudelsky \& Nash, 2002; Hekmatpanah \& Peroutka, 1990; Hysek et al., 2011; Iravani, Asari, Patel, Wieczorek, \& Kruk, 2000; Nash \& Brodkin, 1991; Rudnick \& Wall, 1992). MDMA also inhibits monoamine oxidase, enzymes that are involved in the catabolism on DA, 5-HT, and NE (Leonardi \& Azmitia, 1994; Scorza et al., 1997).

The end result is substantially increased synaptic concentrations of monoamines. Measurement of extracellular concentrations of neurotransmitters via in vivo microdialysis has shown that acute MDMA administration results in dose-dependent increases in extracellular concentrations of 5-HT, DA, and NE (Baumann, Clark, Franken, Rutter, \& Rothman, 2008; Baumann, Clark, \& Rothman, 2008; Kankaanpää, Meririnne, Lillsunde, \& Seppälä, 1998; Kurling, Kankaanpää, \& Seppälä, 2008; Nair \& Gudelsky, 2004; O’Shea et al., 2005; Shankaran \& Gudelsky, 1999). Because MDMA has the greatest affinity for the 5-HT transporter, these effects are primarily exerted on the 5-HT system (Battaglia, Brooks, et al., 1988). Depending on the dose and the brain region assessed, the effect of acute MDMA administration on extracellular concentrations of 5-HT is typically much greater than the effect on DA (see Schenk, 2011). As will be discussed below, this is not a pharmacological profile that is typical of a drug of abuse.

\section{Role of serotonin in self-administration}

As previously mentioned, considerable evidence indicates that DAergic mechanisms positively mediate the reinforcing effects of drugs and their self-administration by laboratory 
animals. In contrast, several lines of evidence suggest that drug-produced increases in 5HTergic neurotransmission is not reinforcing and is indeed inhibitory to self-administration. Firstly, humans do not abuse drugs that selectively increase synaptic concentrations of 5-HT, such as selective serotonin reuptake inhibitors (SSRI; a class of anti-depressant), nor are such drugs self-administered by laboratory animals (Götestam \& Andersson, 1975; Howell \& Byrd, 1995; D. C. S. Roberts et al., 1999; Tessel \& Woods, 1975; Vanover, Nader, \& Woolverton, 1992).

Secondly, for drugs that have both DAergic and 5-HTergic effects (i.e. most psychostimulants), those with both greater DAergic effects and smaller 5-HTergic effects are more efficacious reinforcers. For example, amphetamine or cocaine analogues that had greater binding affinities for the DA transporter relative to the 5-HT transporter were more effective at maintaining self-administration (Ritz \& Kuhar, 1989; D. C. S. Roberts et al., 1999). Conversely, drugs that were more potent in vivo releasers of 5-HT, but had equal potencies as DA releasers, were less effective at maintaining self-administration (Wee et al., 2005).

Thirdly, manipulations that increase or decrease 5-HTergic activity have the opposite effect on self-administration. For example, increasing 5-HT levels by the administration of the synthetic precursor of 5-HT, L-tryptophan, decreased the self-administration of cocaine (Carroll, Lac, Asencio, \& Kragh, 1990b; McGregor, Lacosta, \& Roberts, 1993) and amphetamine (Lyness, 1983; J. E. Smith, Dianna, Smith, Leccese, \& Lyness, 1986). Conversely, inhibition of 5-HT synthesis with p-chlorophenylalanine (pCPA), or 5-HT depletion achieved by 5,7-dihydroxytryptamine (5,7-DHT) lesions, facilitated the selfadministration of amphetamine (Fletcher, Korth, \& Chambers, 1999; Leccese \& Lyness, 1984; Lyness, Friedle, \& Moore, 1980), MDMA (Bradbury et al., 2013), ethanol (Lyness \& Smith, 1992), and morphine (Dworkin, Guerin, Co, Smith, \& Goeders, 1988; J. E. Smith, Shultz, Co, Goeders, \& Dworkin, 1987). Administration of various 5-HT agonists also decreased the selfadministration of cocaine (Carroll, Lac, Asencio, \& Kragh, 1990a; Carroll et al., 1990b; Czoty, Ginsburg, \& Howell, 2002; Peltier \& Schenk, 1993; Richardson \& Roberts, 1991), amphetamine (Dianna, Smith, Smith, \& Lyness, 1986; Porrino et al., 1989; Wee \& Woolverton, 2006), methamphetamine (Munzar et al., 1999), ethanol (Lyness \& Smith, 1992), and heroin (Higgins, Wang, Corrigall, \& Sellers, 1994; Y. Wang, Joharchi, Fletcher, Sellers, \& Higgins, 1995). In one study, amphetamine was mixed with various doses of the 5-HT releasing agent, fenfluramine, and the rate of self-administration of the mixture was inversely correlated with the dose of fenfluramine (Wee \& Woolverton, 2006). 
5-HT projections originating from the dorsal and median raphe innervate several brain regions that have been implicated in addiction, including the NAcc, VTA, and PFC (Di Matteo, Di Giovanni, Pierucci, \& Esposito, 2008). 5-HTergic mechanisms can modulate DA neurotransmission by direct activation of 5-HT receptors located on DA neurons or via inhibitory gamma-aminobutyric acid (GABA) and excitatory glutamate intermediary connections (Bankson \& Cunningham, 2001; Di Matteo et al., 2008; Gudelsky \& Yamamoto, 2008). 5-HT-mediated inhibition of DA release has been suggested to be one mechanism by which increased 5-HT attenuates the reinforcing effects of drugs since increasing synaptic 5HT concentrations with an SSRI, administered at doses that decreased cocaine selfadministration, also decreased cocaine-produced increases in extracellular DA in the caudate nucleus (Czoty et al., 2002). It should be noted that the relationship between 5-HT and DA is complicated, however. There are at least 14 distinct 5-HT receptor subtypes (Barnes \& Sharp, 1999) and activation of some of these receptors has been shown to facilitate DA release (5$\left.\mathrm{HT}_{1 \mathrm{a}}, 5-\mathrm{HT}_{1 \mathrm{~b}}, 5-\mathrm{HT}_{2 \mathrm{a}}, 5-\mathrm{HT}_{2 \mathrm{~b}}\right)$, whereas activation of others $\left(5-\mathrm{HT}_{2 \mathrm{c}}\right)$ has been shown to inhibit DA release (reviewed by Di Matteo et al., 2008).

Given the pharmacology of MDMA, and the apparent inhibitory role of 5-HT in the reinforcing effects of drugs of abuse, why then is MDMA self-administered by laboratory animals, and why is it abused by humans?

\section{Effect of repeated MDMA exposure on serotonin and dopamine}

The answer to this question lies in an understanding of the changes in both 5-HTergic and DAergic mechanisms that occur with repeated MDMA exposure. Repeated exposure to MDMA has long been known to produce substantial deficits in 5-HT neurotransmission. In rats, repeated experimenter-administered injections of MDMA $(10-40 \mathrm{mg} / \mathrm{kg}$, twice daily, for 4 days) produced long-lasting and dose-dependent reductions in 5-HT reuptake sites as well brain tissue levels of 5-HT and 5-hydroxyindoleacetic acid (5-HIAA), a metabolite of 5-HT (Battaglia, Yeh, \& De Souza, 1988; Battaglia et al., 1987; Commins et al., 1987; O’Hearn, Battaglia, De Souza, Kuhar, \& Molliver, 1988). Both baseline and MDMA-produced increases in extracellular striatal 5-HT were also decreased following repeated MDMA exposure (10 $\mathrm{mg} / \mathrm{kg}$, every 2 hours, for a total of 4 injections; Shankaran \& Gudelsky, 1999). These effects were prevented by the co-administration of the SSRIs, citalopram (Battaglia, Yeh, et al., 1988), or fluoxetine (Schmidt, 1987), suggesting that MDMA-produced deficits are due to the potent effect of MDMA on the 5-HT system. Similar deficits have also been observed in non-human primates following repeated MDMA/ecstasy exposure (Insel, Battaglia, Johannessen, Marra, 
\& De Souza, 1989; Kleven, Woolverton, \& Seiden, 1989; Ricaurte, DeLanney, Irwin, \& Langston, 1988; Wilson, Ricaurte, \& Molliver, 1989), and can take several years to fully recover (Ricaurte, Martello, Katz, \& Martello, 1992).

The relevance of some of these findings to human MDMA/ecstasy use has been questioned, however, due to the use of relatively high doses of non-contingent MDMA (Baumann, Wang, \& Rothman, 2007; de la Torre \& Farré, 2004; Ricaurte, McCann, Szabo, \& Scheffel, 2000). More recent studies investigating the effect of self-administered MDMA offer greater external validity and have shown similar results. In rats, lower densities of 5-HT reuptake sites (Schenk et al., 2007) and persistent decreases in 5-HT tissue levels (Do \& Schenk, 2013) were found following MDMA self-administration $(365-440 \mathrm{mg} / \mathrm{kg}$ over 17 days). MDMA-produced increases in extracellular NAcc 5-HT were also decreased following 20 days of MDMA self-administration ( 100 mg/kg over 20 days; Reveron et al., 2010). 5HTergic deficits appear to be dependent on exposure levels, however, since self-administration of smaller amounts of MDMA failed to significantly decrease densities of 5-HT reuptake sites or 5-HT tissue levels in rats (Do \& Schenk, 2013) or rhesus monkeys (Banks et al., 2008; Fantegrossi et al., 2004). Some 5-HT deficits have also been found in human ecstasy users (McCann, Ridenour, Shaham, \& Ricaurte, 1994; McCann, Szabo, Scheffel, Dannals, \& Ricaurte, 1998; Semple, Ebmeier, Glabus, O’Carroll, \& Johnstone, 1999; Thomasius et al., 2003; Verkes et al., 2001), and these appear to persist for several years (Thomasius et al., 2006).

While it is clear that repeated MDMA exposure can result in various 5-HTergic deficits, some studies have found that repeated MDMA exposure can sensitise DAergic mechanisms. In rats, tissue levels of DA in the NAcc were increased four weeks after repeated MDMA exposure (20 mg/kg/day for 10 days; Mayerhofer, Kovar, \& Schmidt, 2001). MDMA- and cocaine-produced increases in extracellular NAcc DA were also enhanced following repeated MDMA exposure (20 mg/kg/day for 4 days; Kalivas et al., 1998; Morgan, Horan, Dewey, \& Ashby, 1997). Intermittent dosing appears critical to the development of these sensitised DAergic responses, however, as when similar amounts of MDMA was administered over a single day, there was no effect (Shankaran \& Gudelsky, 1999). Rats that acquired MDMA selfadministration (165 mg/kg over an average of 21 days) also showed enhanced MDMAproduced $(10 \mathrm{mg} / \mathrm{kg})$ increases extracellular striatal DA (Colussi-Mas et al., 2010). Selfadministration of lesser amounts of MDMA ( 100 mg/kg over 20 days) failed to impact MDMA-produced (3 mg/kg) increases in extracellular NAcc DA, however (Reveron et al., 2010). These results suggest that both the total amount of exposure and the pattern of exposure 
mediate the development of sensitised DAergic mechanisms following repeated MDMA exposure.

Repeated intermittent MDMA exposure also has been shown to result in behavioural sensitisation (Ball et al., 2006; Bradbury, Gittings, \& Schenk, 2012; Kalivas et al., 1998; Spanos \& Yamamoto, 1989). Consistent with other psychostimulant drugs, MDMA-induced behavioural sensitisation appears to involve sensitised DAergic mechanisms. MDMA-induced behavioural sensitisation was accompanied by enhanced MDMA-produced increases in NAcc DA (Kalivas et al., 1998), increased dendritic spine density in DA terminal regions (Ball et al., 2009), and was prevented by co-treatment with the $\mathrm{DA}_{2}$ receptor antagonist, eticlopride (van de Wetering \& Schenk, 2017). Cross-sensitisation to the behavioural effects of other DAergic drugs including cocaine, amphetamine, and the $\mathrm{DA} \mathrm{D}_{2}$ agonist, quinpirole, has also been demonstrated (Kalivas et al., 1998; Schenk \& Bradbury, 2015).

\section{Serotonin, dopamine, and MDMA self-administration}

Like other drugs of abuse, increased 5-HT neurotransmission appears to be inhibitory to the reinforcing effects of MDMA and has been shown to limit the acquisition of MDMA self-administration. Rats with greater MDMA-produced increases in extracellular NAcc 5-HT were less likely to subsequently acquire MDMA self-administration (Bradbury et al., 2013). In contrast, manipulations that impaired 5-HT neurotransmission facilitated the acquisition of MDMA self-administration and rendered the acquisition profile more comparable to that of other psychostimulant drugs of abuse. For example, neurotoxic 5,7-DHT lesions (Bradbury et al., 2013) or a genetic mutation of the 5-HT transporter (Oakly et al., 2013) substantially facilitated the acquisition of self-administration, decreasing the latency to acquire and increasing the percentage of rats that met acquisition criteria. Once MDMA self-administration has been acquired, however, it appears 5-HT plays a limited role, since the administration of various 5-HT antagonists did not affect the maintenance of MDMA self-administration (Schenk et al., 2016).

Like other drugs of abuse, evidence suggests that the reinforcing effects of MDMA are mediated by DAergic mechanisms. In rhesus monkeys, the potency of the two isomers of MDMA, S(+)-MDMA and R(-)-MDMA, as well as the racemic mixture, $( \pm)$-MDMA, to function as reinforcers and maintain self-administration was positively correlated with the varying potency of each drug to stimulate DA release (Johnson, Hoffman, \& Nichols, 1986; Z. Wang \& Woolverton, 2007). In rats, treatment with various DA receptor antagonists produced a rightward shift in the dose-response curve for MDMA self-administration (Brennan et al., 
2009; Daniela et al., 2004). Moreover, rats that met acquisition criteria for MDMA selfadministration displayed greater MDMA-produced increases in extracellular striatal DA compared to those that failed to acquire self-administration (Colussi-Mas et al., 2010). These results suggest that the development of a sensitised DAergic response might be crucial for the acquisition of MDMA self-administration. In support of this idea, pretreatment with MDMA under conditions that produced sensitisation to the locomotor activating effects of MDMA also facilitated the acquisition of MDMA self-administration, decreasing the latency to acquire and increasing the proportion of rats that met acquisition criteria (van de Wetering \& Schenk, 2017).

Collectively, these findings suggest that repeated MDMA exposure can result in both 5-HTergic deficits and DAergic sensitisation. These neuroadaptations, for the reasons discussed above, would be expected to enhance the reinforcing efficacy of MDMA. Thus, it has been hypothesised that the acquisition of MDMA self-administration and the subsequent escalation of MDMA-intake progress as a function of these neuroadaptations, and with repeated exposure, the pharmacological and behavioural profile of MDMA becomes increasingly similar to other psychostimulant drugs of abuse such as amphetamine or cocaine (Schenk, 2011).

\section{The current thesis}

The purpose of the current research was to further investigate the neuroadaptations that develop with repeated MDMA exposure in rats. Initial experiments aimed to identify the specific brain regions that undergo behaviourally relevant neuroadaptive change, as measured by $\Delta$ FosB immunohistochemistry (IHC). Subsequent experiments aimed to investigate the more specific neurochemical changes within these brain regions, as measured by in vivo microdialysis and liquid chromatography-mass spectrometry (LC-MS).

\section{$\Delta$ FosB and immunohistochemistry}

Drug-craving and vulnerability to relapse can persist even after decades of abstinence. This suggests that the various drug-induced neuroadaptations that underlie drug-craving and relapse are equally persistent. Thus, an important focus of addiction research recently has been to investigate the mechanisms by which these maladaptive neuroadaptations are maintained over such long periods of time, even after drug exposure has ceased.

Recent evidence has highlighted the important role of changes in gene expression in mediating the brain changes induced by repeated drug exposure (Madsen, Brown, \& Lawrence, 2012; Mews, Walker, \& Nestler, 2019; Robison \& Nestler, 2011). Gene expression is the 
process by which the genetic information encoded on a sequence of DNA is synthesised into a functional cellular product, such as a protein. The first step of this process, termed transcription, is where a particular segment of DNA is copied into mRNA by the enzyme, RNA polymerase. In the next phase, termed translation, the messenger RNA is used as the blueprint to form an amino-acid chain that can be later folded into a functional protein.

One mechanism by which repeated drug exposure can influence gene expression is by increasing or decreasing the activity or number of various transcription factors (Madsen et al., 2012; Mews et al., 2019; Robison \& Nestler, 2011). Transcription factors are specialised proteins or complexes of multiple proteins, that function to regulate the transcription process. They bind to target sequences of DNA and increase or repress the transcription of the encoded gene by respectively promoting or blocking RNA polymerase activity. Transcription factors are one of the mechanisms that ensure that genes are expressed at the right time, in the right amount, and in the right cell.

While several transcriptions factors have been implicated in drug addiction (see Mews et al., 2019; Nestler, 2012; Robison \& Nestler, 2011), $\Delta$ FosB is one such transcription factor that, for the reasons discussed below, may play a particularly important role in the development and maintenance of drug addiction. Like other Fos family proteins, $\Delta$ FosB dimerises with Jun family proteins to form the active transcription factor complex, activator protein-1 (AP-1), which binds to AP-1 sites on the promoter regions of specific genes to regulate their transcription. All Fos family proteins are induced very rapidly in the brain in response to a wide variety of environmental stimuli such as drug exposure or stress (Mews et al., 2019; Nestler, 2008; Robison \& Nestler, 2011). The induction of these proteins is typically transient, however, with protein levels returning to baseline within a few hours. $\Delta$ FosB is unique compared to other Fos family proteins, and indeed most other transcription factors, in that certain isoforms of the protein can persist in the brain for several weeks or even months due to their particularly high stability (Alibhai, Green, Potashkin, \& Nestler, 2007; Carle et al., 2007; Chen, Kelz, Hope, Nakabeppu, \& Nestler, 1997; Ulery, Rudenko, \& Nestler, 2006). This allows $\Delta$ FosB (the stable isoforms of) to accumulate in very high levels following repeated drug exposure and to remain at these elevated levels for long periods of time after drug exposure has ceased. It has therefore been suggested that $\Delta$ FosB-mediated transcription is an important mechanism by which repeated drug exposure can produce persistent brain changes that underlie certain aspects of addiction (McClung et al., 2004; Nestler, 2008; Nestler, Kelz, \& Chen, 1999). 
Chronic exposure to various experimenter- (Brenhouse \& Stellar, 2006; Conversi, Bonito-Oliva, Orsini, Colelli, \& Cabib, 2007; De Pauli et al., 2014; Hope et al., 1994; Kaplan, Leite-Morris, Fan, Young, \& Guy, 2011; Kaste, Kivinummi, Piepponen, Kiianmaa, \& Ahtee, 2009; Y. Kim et al., 2009; Marttila, Raattamaa, \& Ahtee, 2006; McDaid, Graham, \& Napier, 2006; Moratalla, Elibol, Vallejo, \& Graybiel, 1996; Muller, Unterwald, \& Fujimoto, 2005; Nye, Hope, Kelz, Iadarola, \& Nestler, 1995; Perrotti et al., 2005, 2008) or self-administered (Cornish, Hunt, Robins, \& McGregor, 2012; Krasnova et al., 2013; Larson et al., 2010; Perrotti et al., 2005, 2008; Pich et al., 1997; Winstanley et al., 2007) drugs of abuse have been shown to produce pronounced increases in $\triangle \mathrm{FosB}$ in various brain regions, as measured by Western blot or IHC. The most pronounced increases induced by repeated drug exposure are typically observed within $\mathrm{D}_{1}$-type MSN of the NAcc and other parts of the striatum, brain regions that play crucial roles in mediating reinforcement. Several studies have also found increased $\Delta$ FosB following repeated drug exposure in numerous other brain regions implicated in addiction, including the PFC and amygdala.

The behavioural effects of increased $\Delta$ FosB within some of these brain regions has been demonstrated by studies that have manipulated $\Delta$ FosB expression using viral-mediated gene transfer or bitrangenic animals. Overexpression of $\Delta \mathrm{FosB}$ in the NAcc and surrounding striatal areas increased both cocaine- (Kelz et al., 1999) and morphine- (Zachariou et al., 2006) produced CPP as well as cocaine-produced locomotor activity (Grueter, Robison, Neve, Nestler, \& Malenka, 2013; Kelz et al., 1999). In contrast, local overexpression of $\Delta$ c-Jun, which antagonises $\triangle$ FosB-mediated transcription, reduced cocaine CPP (Peakman et al., 2003). Following repeated morphine exposure, overexpression of $\Delta \mathrm{FosB}$ in the striatum also enhanced the development of physical dependence and withdrawal symptoms (Zachariou et al., 2006). Importantly, striatal overexpression of $\Delta \mathrm{FosB}$ also facilitated the acquisition of cocaine selfadministration and increased responding maintained by cocaine under PR schedules (Colby, Whisler, Steffen, Nestler, \& Self, 2003). In the OFC, overexpression of $\Delta$ FosB mimicked the effect of repeated cocaine exposure on rodent tests of attention and decision making while overexpression of $\Delta \mathrm{JunD}$, which also antagonises $\Delta \mathrm{FosB}$-mediated transcription, prevented the effect of repeated cocaine exposure on these tests (Winstanley et al., 2007). Overexpression of $\triangle \mathrm{FosB}$ in the OFC also sensitised the locomotor activating effects of cocaine (Winstanley et al., 2009). These findings indicate that $\Delta$ FosB expression alone can result in the development of several relevant behavioural phenotypes that are also consequences of repeated drug exposure (Nestler, 2008; Robison \& Nestler, 2011). 
Progress has been made in identifying the specific transcriptional mechanisms by which $\Delta$ FosB can induce long-term brain changes that lead to the development of these phenotypes. $\Delta$ FosB has been shown to mediate the transcription of several genes/proteins that regulate structural plasticity and dendritic spine formation of striatal MSN. These include activityregulated cytoskeleton-associated protein (ARC), cyclin-dependent kinase 5 (CDK5), p35, nuclear factor-kappaB (NF-kB), synaptotagmin, microtubule-associated proteins, actin-related proteins, and kinesin (Bibb et al., 2001; Maze et al., 2010; Nestler, 2008, 2012; Robison \& Nestler, 2011; Winstanley et al., 2009). Indeed, overexpression of $\Delta$ FosB in the striatum has been shown to increase the number and density of dendritic spines in $\mathrm{D}_{1}$-type MSN, and this was associated with sensitised behavioural responses to cocaine (Grueter et al., 2013). $\Delta$ FosB also regulates the transcription of several genes/proteins involved in glutamatergic synaptic function and plasticity including the $\alpha$-amino-3-hydroxy-5-methyl-4-isoxazolepropionic acid (AMPA) receptor subunit, GluR2, as well as CDK5, p35, and $\mathrm{Ca}^{2+} /$ calmodulin-dependent protein kinase II (CaMKII; Bibb et al., 2001; Kelz et al., 1999; Nestler, 2012). These genes have been implicated in the rewarding effects of psychostimulants (Kauer \& Malenka, 2007; Kelz et al., 1999; T. E. Robinson \& Kolb, 2004) and were upregulated by $\Delta$ FosB overexpression or repeated cocaine exposure, an effect that was blocked by $\Delta c$-Jun overexpression (Bibb et al., 2001; Kelz et al., 1999; McClung \& Nestler, 2003; Peakman et al., 2003). $\Delta$ FosB also mediates the expression of dynorphin, an opioid peptide, which regulates DAergic output from the VTA (Bruchas, Land, \& Chavkin, 2010; Zachariou et al., 2006). Enhanced morphine dependence and withdrawal symptoms induced by $\Delta \mathrm{FosB}$ overexpression were shown to be mediated by dynorphin (Zachariou et al., 2006). Finally, $\Delta$ FosB also regulates the expression of several other transcription factors, including NF-kB, which has been implicated in dendrite formation as well as the neurotoxic effects of methamphetamine (Ang et al., 2008; Shah, Silverstein, Singh, \& Kumar, 2012), and c-Fos, another Fos family protein (Renthal et al., 2008; Robison \& Nestler, 2011).

It is clear that $\Delta$ FosB mediates an array of neural mechanisms that are known to be to be relevant to drug-taking and drug-seeking behaviour. The accumulation of $\Delta \mathrm{FosB}$ in the brain has, therefore, provided a useful marker to identify brain regions that have undergone relevant neuroadaptive change following repeated drug exposure (Nestler, 2008; Robison \& Nestler, 2011). The effect of repeated MDMA exposure on $\triangle F$ FosB expression has not yet been determined, however. The first aim of the current research was, therefore, to map the accumulation of $\triangle F$ FosB across the brain following chronic MDMA self-administration in rats. 
Two procedures are typically used to measure $\Delta$ FosB in the brain; Western blot (immunoblotting) and IHC. For the purpose of mapping the accumulation of $\Delta$ FosB across the brain, IHC offers much greater spatial resolution, as $\Delta \mathrm{FosB}$ can be measured in as many discrete brain regions on a tissue slice as needed. IHC involves the detection of target antigens (proteins) by labelling them with specific antibodies (Ramos-Vara, 2005). Visualisation of the antibody-antigen interaction can be achieved by labelling the antibody with an enzyme that can catalyse a colour producing reaction, or with a fluorescent fluorophore. Each coloured or fluorescent cell can then be counted under a microscope. Often, an un-labelled primary antibody is used to which to one or more secondary antibodies are subsequently bound that can host larger labelling complexes in order to increase the intensity of the staining/fluorescence (Figure 1.2).

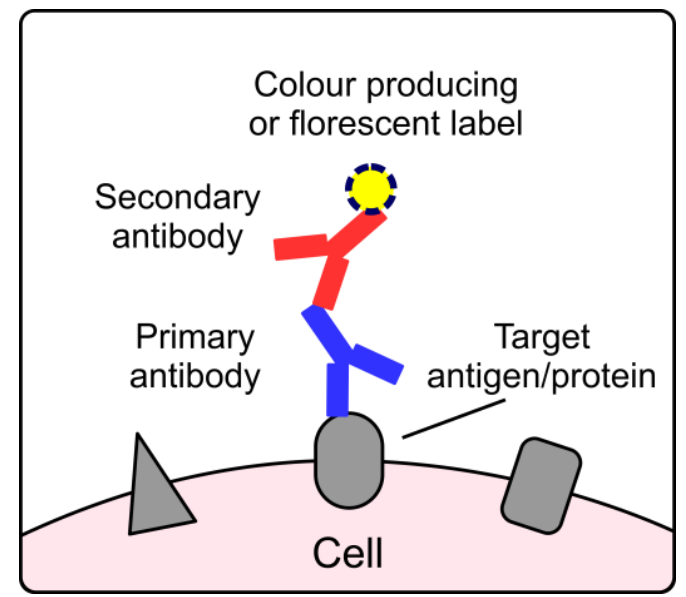

Figure 1.2. Basic principle of immunohistochemistry. The target antigen/protein is labelled with a specific primary antibody, to which a secondary antibody can be also be bound to improve detection. The secondary antibody is labelled with an enzyme that can catalyse a colour producing reaction or with a fluorescent fluorophore, which can then be viewed under a microscope. 


\section{Microdialysis and LC-MS}

Mapping the expression of $\triangle \mathrm{FosB}$ throughout the brain following repeated drug exposure can identify brain regions that have undergone neuroadaptive change. However, this procedure cannot provide any information on the specific drug-produced neurochemical adaptations that may take place within these brain regions. A number of pre-clinical procedures have been developed to quantify these neurochemical changes. Microdialysis is one such technique that allows for the collection of samples from extracellular fluid in vivo (Chefer, Thompson, Zapata, \& Shippenberg, 2009; Ungerstedt \& Pycock, 1974; Westerink \& Cremers, 2007). A probe with a semipermeable membrane is inserted into the brain area of interest and perfused with artificial cerebrospinal fluid (aCSF; Figure 1.3A). Neurotransmitters, metabolites, and other small molecules diffuse across the membrane down their concentration gradient and into the perfusate (Figure 1.3B). The dialysate is then collected and can be subsequently analysed by various means (Figure 1.3C).

Microdialysis has the advantage of providing some temporal information since samples can be collected every 5-30 min. Microdialysis is also advantageous compared to some other neurochemical assays in that only the extracellular fluid is sampled, and it is the extracellular concentration of neurotransmitters or metabolites that are of interest for neurotransmission. Importantly, microdialysis also permits neurochemical samples to be collected from awake, freely moving subjects, allowing for the concurrent collection of behavioural data. When coupled with appropriate behavioural and analytic procedures, microdialysis can provide an effective means of measuring the neurochemical correlates of drug-produced behaviour (Chefer et al., 2009; Davies, 1999; Westerink, 1995; Westerink \& Cremers, 2007). 


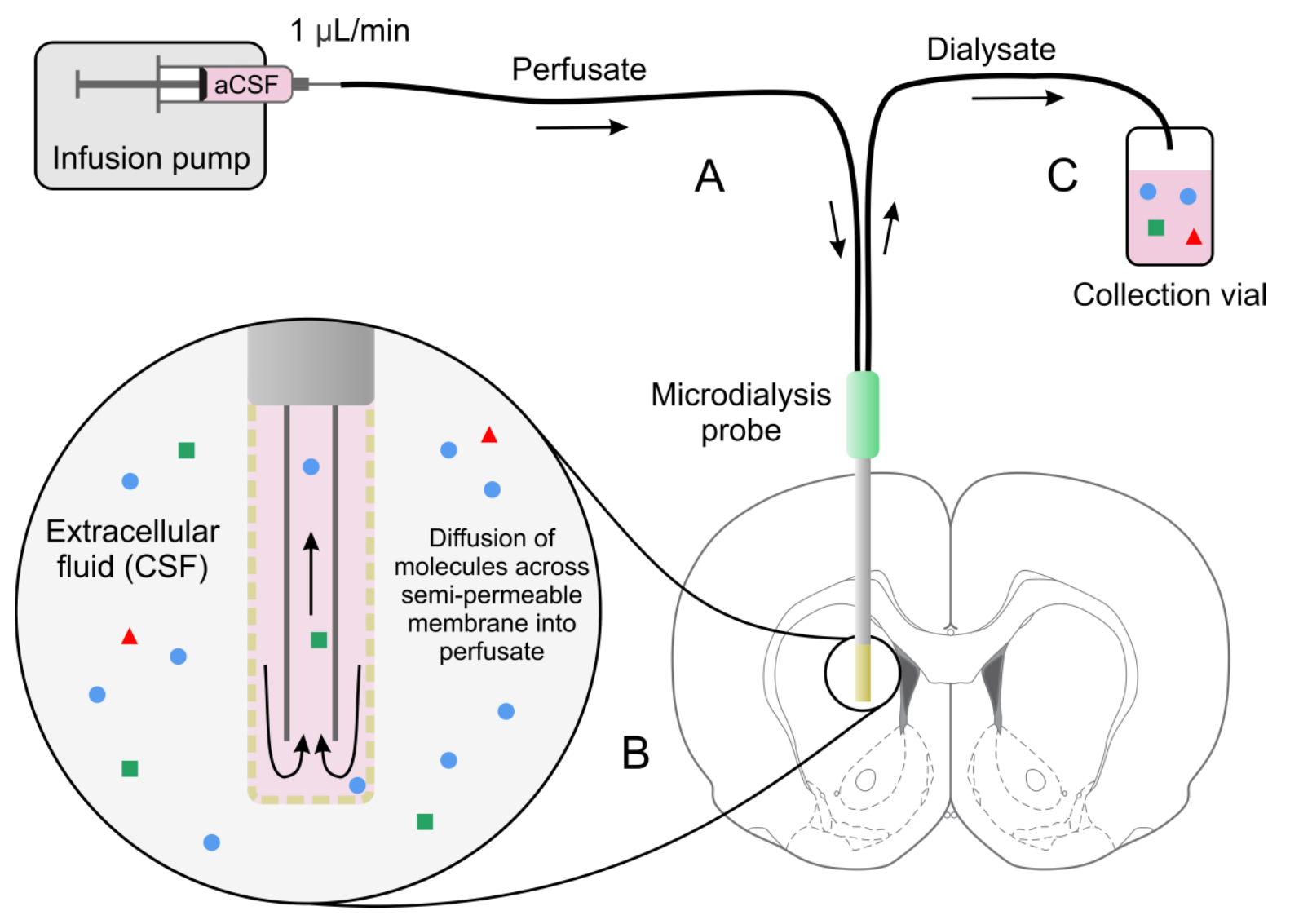

Figure 1.3. Basic principle of microdialysis. A) Perfusate consisting of artificial cerebrospinal fluid $(\mathrm{aCSF})$ is pumped at a constant flow rate through a microdialysis probe implanted in the brain region of interest. B) At the tip of the probe, a semi-permeable membrane allows for the diffusion of molecules present in extracellular fluid into the perfusate. C) The perfusate, now dialysate, continues to flow into a collection vial where the contents can be subsequently analysed. 
High-performance liquid chromatography (HPLC) methods are the most common procedures used to analyse dialysate samples collected via in vivo microdialysis (Davies, Cooper, Desmond, Lunte, \& Lunte, 2000). HPLC involves passing solvents containing the dialysate sample through a separation column under high pressure (Figure 1.4A). Each compound/component of the sample interacts differently with the adsorbent material within the column, which leads to the physical separation of the sample components as they flow through the column over time (Figure 1.4B). Using various chromatographic detection methods, the relative amount of each component of the sample mixture can be determined according to the time at which it emerged from the column (retention time; Figure 1.4C). This produces a chromatogram with peaks representing the relative amounts of the various sample components (Figure 1.4D). To quantify a particular compound/sample component, the height or area of the chromatographic peaks and their retention times are compared to those produced by samples containing known amounts of the compound of interest (i.e. calibration standards).

The most common chromatographic detection methods involve electrochemical, ultraviolet, or fluorescence detection (Swartz, 2010). These detection methods can be limited by the fact that they often need to be specifically tailored for the analysis of a particular compound or class of compound. The right combination of solvents, column specifications, and other parameters must be chosen to ensure that the target compound(s) are adequately separated and detected. Thus, multiple samples may need to be collected, and the analysis may need to be re-run for each compound of interest. The duration of the analysis may also need to be very long in order to ensure that all detectable sample components are adequately separated and do not co-elute, which would prevent quantification 


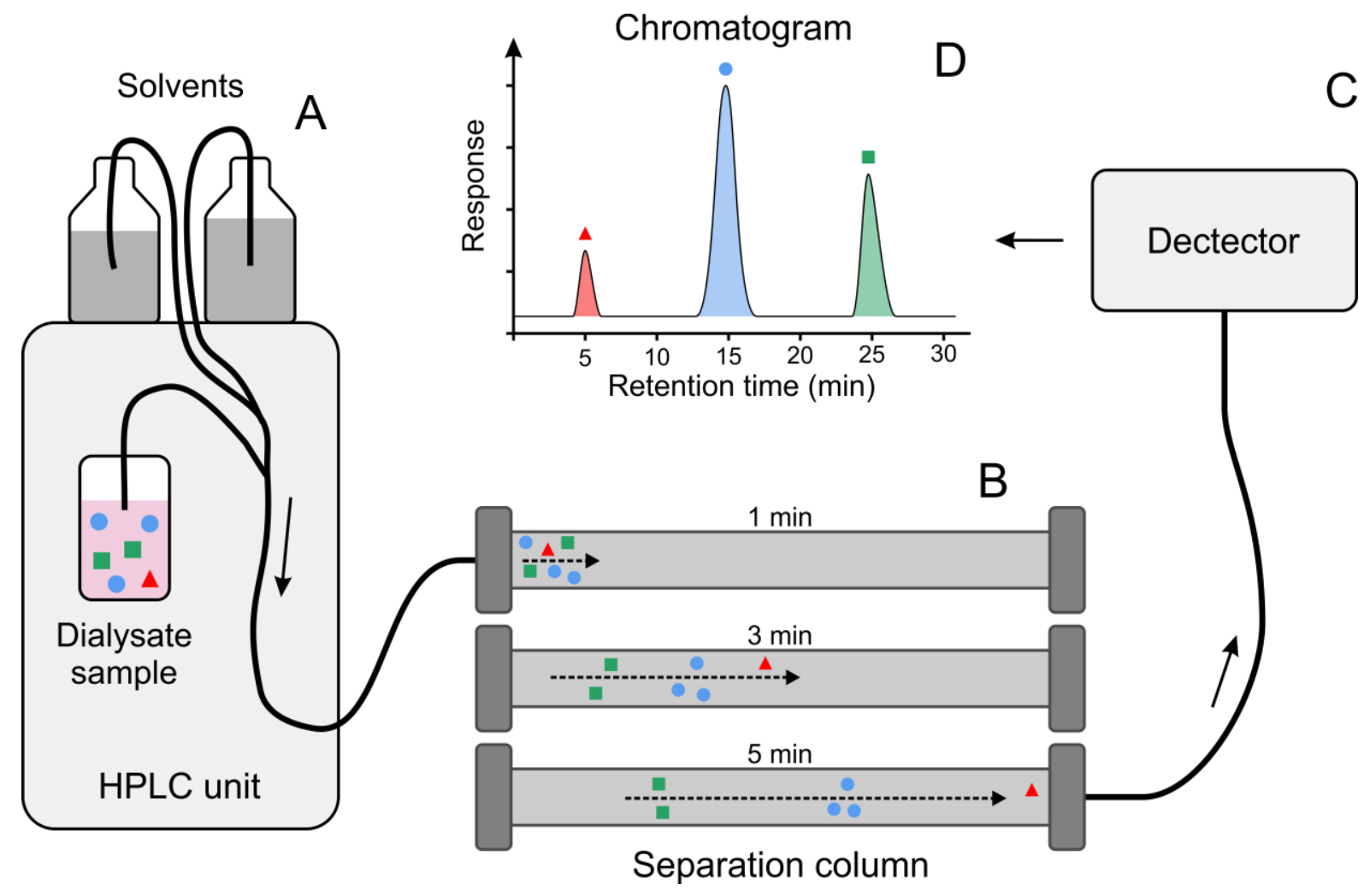

Figure 1.4. Basic principle of high-performance liquid chromatography (HPLC). A) The sample is mixed with various solvents under high pressure into a separation column. B) Each component of the sample mixture interacts differently with the adsorbent material within the column, which leads to the physical separation of the sample components as they flow through the column over time. C) Using various chromatographic detection methods, the relative amount of each component of the sample mixture is measured. D) This produces a chromatogram with peaks representing the relative amount of each sample component according to time at which it emerged from the column (retention time). 
Another detection method for HPLC is mass spectrometry (MS; Niessen, 2006). The first step in MS detection involves the ionisation of the sample compounds as they elute from the column. The compounds, once ionised, are then further separated according to their massto-charge ratio $(\mathrm{m} / \mathrm{z})$. Lastly, the relative abundance of each ion is measured and recorded as a mass spectrum; a two-dimensional visualisation of the relative abundance of each ion according to their $\mathrm{m} / \mathrm{z}$ (Figure $1.5 \mathrm{~A}$ ). Mass spectrum are typically recorded several times per second, and thus the total data set may include hundreds to thousands of sequential mass spectrum as a function of retention/acquisition time. The total ion chromatogram (TIC) represents the total abundance of all measured ions as a function of retention time (Figure 1.5B), while an extracted ion chromatogram (EIC) represents the abundance of ions of a particular $\mathrm{m} / \mathrm{z}$ as a function of retention time (Figure 1.5C). To quantify a particular compound, an EIC is generated for the target ion and the peak height or area is compared to calibration standards. A three-dimensional representation of LC-MS data is shown in Figure 1.5D.

HPLC coupled with MS detection (LC-MS) has several advantages over other detection methods. Firstly, imperfections in chromatographic separation, such as multiple compounds having the same retention time, are less of an issue due to the further separation and measurement of compounds based on their $\mathrm{m} / \mathrm{z}$ (see Figure 1.5D). This improves analytical selectivity, allowing for the quantification of several sample components in a single analysis, and leads to shorter analytic timeframes since adequate chromatographic separation can be achieved in shorter time periods. Secondly, MS detectors generally have much better sensitivity, providing lower detection limits, which allows for the use of smaller samples volumes and/or the analysis of compounds present in lower concentrations. Thirdly, analytical specificity can be increased by using tandem mass spectrometry (MS/MS), which predictably fragments ions into smaller structures in order to provide information that can be used to help determine the identity of a compound. Finally, due to the increased selectivity of LC-MS, the sample preparation and chromatography procedures can be much more inclusive, which allows for metabolomics analysis; an unbiased, global analysis of all small molecules/metabolites within a biological system/sample. This type of analysis is typically not hypothesis-driven, but rather hypothesis-generating, being able to potentially identify unexpected or novel compounds of interest. These advantages of LC-MS have yet to be fully exploited for the study of the neurobiology of drug addiction. The current research, therefore, aimed to develop and employ a novel procedure that exploits the combined advantages of microdialysis and LC-MS in order to study the effects of repeated MDMA exposure. 

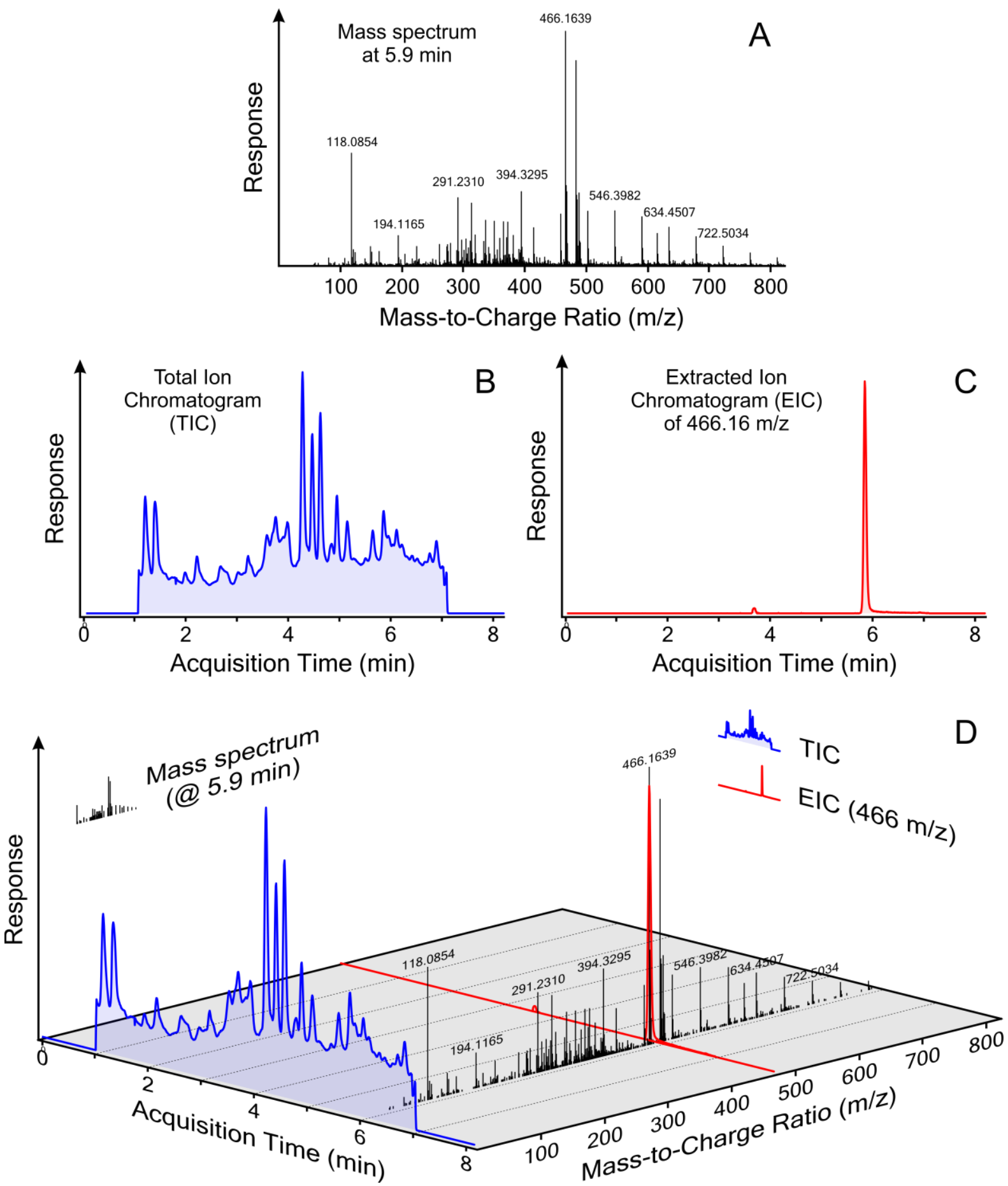

Figure 1.5. Data obtained by liquid chromatography-mass spectrometry (LC-MS). A) Example mass spectrum at 5.9 min acquisition time showing the relative response/abundance of ions as a function of their mass to charge ratio $(\mathrm{m} / \mathrm{z})$. B) Total ion chromatogram (TIC) shows the total response/abundance of all ions as a function of retention/acquisition time. C) Example extracted ion chromatogram (EIC) showing the response/abundance of $466.16 \mathrm{~m} / \mathrm{z}$ as a function of retention/acquisition time. D) Three-dimensional representation of LC-MS data showing the TIC as well as an example mass spectrum and EIC. 


\section{Aims and objectives}

MDMA/ecstasy is a popular recreational drug, and some users exhibit symptoms characteristic of a SUD. Despite this, the behavioural and neurobiological effects of repeated MDMA exposure are less well understood than other drugs of abuse. The purpose of the current research was to investigate the neuroadaptations that develop with repeated MDMA exposure in laboratory rats.

First, the brain regions that undergo significant neuroadaptive change following chronic MDMA self-administration were identified by measuring the accumulation of $\Delta$ FosB across multiple brain regions relevant to addiction using IHC. It was expected that MDMA selfadministration would result in a significant accumulation of $\Delta \mathrm{FosB}$ in the NAcc, dorsal striatum, amygdala, and PFC, as has been shown with other drugs of abuse.

Second, the behavioural relevance of the region-specific differences in $\Delta$ FosB accumulation was determined by examining the effect of locally infused MDMA into discrete brain regions on locomotor activity following a sensitising regimen of repeated MDMA exposure. It was predicted that behavioural sensitisation would be expressed when MDMA was infused into the same brain regions that also showed enhanced $\Delta \mathrm{FosB}$ accumulation following repeated MDMA exposure.

Third, a targeted investigation of the more specific neurochemical changes within the identified brain regions of interest was carried out. A microdialysis and LCMS based procedure for the collection and quantification of dialysate samples was developed and validated. This procedure was then employed to determine the effect of a sensitising regimen of repeated MDMA exposure on MDMA-produced changes in dialysate concentrations of targeted neurochemicals, notably DA and 5-HT. It was predicted that MDMA-produced increases in dialysate concentrations of DA would be enhanced following a sensitising regimen of repeated exposure, while dialysate concentrations of 5-HT would be decreased.

Finally, a novel, untargeted metabolomics procedure that incorporated time-series metabolomics data as well as behavioural data was developed and validated. This procedure was then used to analyse microdialysis samples and examine the effect of a sensitising regimen of MDMA exposure on the wider metabolome in attempt to identify other neurochemicals of interest that are relevant to MDMA-produced behavioural sensitisation. 


\section{CHAPTER 2: $\triangle$ FOSB AND IMMUNOHISTOCHEMISTRY}

Parts of this chapter have been adapted with licensed permission from work published in Addiction Biology (van de Wetering \& Schenk, 2019).

\section{Introduction}

There is evidence to suggest that certain transcription factors play an important role in maintaining drug-induced neuroadaptations over long periods of time (Mews et al., 2019; Nestler, 2012; Robison \& Nestler, 2011). $\Delta$ FosB is one such transcription factor that has been shown to accumulate in very high-levels within various brain regions relevant to addiction following repeated exposure to drugs of abuse (Nestler, 2008; Perrotti et al., 2008). The effect of repeated MDMA exposure on $\triangle \mathrm{FosB}$ has not yet been investigated, however, and was, therefore, the focus of the current research.

The effect of repeated experimenter-administered MDMA on c-Fos, another transcription factor, has been previously mapped across a wide range of brain areas using IHC (Colussi-Mas \& Schenk, 2008). Induction of c-Fos in only a small sample of the brain regions was correlated with sensitised MDMA-produced behaviour, however. Further, the expression of c-Fos, like most transcription factors, is transient, lasting only a few hours. In contrast, $\Delta$ FosB (certain isoforms) has been demonstrated to be particularly stable, remaining at elevated levels for up to several weeks or months following repeated drug exposure (Alibhai et al., 2007; Carle et al., 2007; Chen et al., 1997; Ulery et al., 2006). It is for this reason that $\Delta$ FosBmediated transcription has been suggested to be a particularly important mechanism by which repeated drug exposure might produce persistent brain changes that underlie certain aspects of addiction (McClung et al., 2004; Nestler, 2008; Nestler et al., 1999).

The effect of repeated drug exposure on brain $\Delta \mathrm{FosB}$ is typically examined following repeated experimenter-administered drug. While some studies have measured brain $\Delta$ FosB following self-administered drugs, few have done so under the long-term and long-access conditions that have been shown to result in an escalation of drug intake and extensive drugseeking behaviour, two measures that have been suggested to be particularly relevant to the study of SUDs (Ahmed, 2011; Edwards \& Koob, 2013). Even fewer studies have mapped the accumulation of $\Delta \mathrm{FosB}$ across numerous brain regions following repeated drug exposure under these conditions. Given these gaps in the literature, the current research aimed to map the accumulation of $\triangle \mathrm{FosB}$ across several brain regions including the $\mathrm{PFC}$, dorsal striatum, ventral 
striatum, amygdala, VTA, and raphe, following an extensive history of long-access MDMA self-administration.

\section{Methods}

\section{Subjects}

Male Sprague-Dawley rats weighing $300-400 \mathrm{~g}$ were used. Rats were bred in the vivarium at Victoria University of Wellington and housed 2-3 per cage until they weighed 250 $-300 \mathrm{~g}$. Thereafter, rats were housed individually. The housing room was kept at a constant temperature $\left(21^{\circ} \mathrm{C}\right)$ and humidity (55\%). A 12-hour light/dark cycle (lights on at 0700 hours) was in effect. Food and water were available ad libitum except during testing. All experimental protocols were approved by the Animal Ethics Committee of Victoria University of Wellington.

\section{Surgical procedures}

Deep anaesthesia was produced by an injection of a solution combining ketamine (90.0 $\mathrm{mg} / \mathrm{kg}$, intraperitoneal [i.p.], Phoenix Pharm, Auckland, New Zealand) and xylazine (9.0 mg/kg, i.p., Phoenix Pharm, Auckland, New Zealand). The anti-inflammatory analgesic, carprofen (5 mg/kg, subcutaneous [s.c.], Zoetis, Auckland, New Zealand) was then administered, and Lacrilube ${ }^{\circledR}$ was applied to both eyes to prevent corneal desiccation. The external jugular vein was isolated and tied off at the anterior end using sterile thread. A Silastic ${ }^{\circledR}$ catheter was then inserted, advanced towards the atrium, and secured in place with sterile thread. The distal end of the catheter, which was fitted with a $2 \mathrm{~cm}$, sealable piece of $22-$ gauge stainless steel tubing, was routed subcutaneously to an exposed part of the skull and fixed in place using four small screws embedded in dental acrylic. Following surgery, Hartmann's solution $(2 \times 5 \mathrm{~mL}$, s.c. $)$ was administered to restore electrolyte balance, and carprofen $(5.0 \mathrm{mg} / \mathrm{kg}$, s.c.) was administered once daily for two days.

\section{Apparatus}

Self-administration testing was conducted in operant chambers equipped with two levers (Med Associates Inc., USA; model ENV-001) set within sound-attenuating boxes. A 20 mL syringe housed in a mechanical pump (Med Associates Inc., USA; model - PHM-100A) was connected through a swivel and tether apparatus to the i.v. catheter. Responses on the active lever resulted in a $0.1 \mathrm{~mL}$ infusion of drug delivered over 12 seconds and the concurrent illumination of a light located above the lever. Responses on the inactive lever were recorded but produced no programmed consequence. Experiments were conducted within a temperature- 
controlled $\left(19-21^{\circ} \mathrm{C}\right)$ darkroom. Drug delivery and data acquisition were controlled by the Med PC software (Med Associates Inc.).

\section{Self-administration}

For self-administration, \pm MDMA HCl (BDG synthesis, New Zealand) was dissolved in a sterile $0.9 \% \mathrm{NaCl}$ (saline) solution containing $3 \mathrm{IU} / \mathrm{mL}$ heparin. Daily 6-hour MDMA selfadministration sessions were conducted six days per week. Prior to each self-administration session, catheters were flushed with $0.2 \mathrm{~mL}$ of sterile $0.9 \% \mathrm{NaCl}$ solution containing heparin (30 IU/mL) and penicillin (250 $000 \mathrm{IU} / \mathrm{mL})$ to prevent blood coagulation and infection. Immediately after each self-administration session, catheters were flushed again with the heparin-penicillin solution. Every seventh day, catheter patency was confirmed by the immediate loss of the righting reflex following the administration of sodium pentobarbital (25 $\mathrm{mg} / \mathrm{kg}$, i.v.).

Each self-administration session commenced with an experimenter-administered infusion to clear the catheter of the penicillin-heparin solution. Thereafter, infusions of $1 \mathrm{mg} / \mathrm{kg}$ MDMA were delivered according to an FR-1 schedule of reinforcement until a total intake of $90 \mathrm{mg} / \mathrm{kg}$ had been self-administered (9 - 14 days). The MDMA infusion dose was then decreased to $0.5 \mathrm{mg} / \mathrm{kg}$ until a total MDMA intake of $350 \mathrm{mg} / \mathrm{kg}$ was reached (20 - 26 days). Thereafter, an additional nine daily self-administration sessions were conducted. Total MDMA intake ranged from $515-692 \mathrm{mg} / \mathrm{kg}$ over a 29 - 35-day period $(n=4)$. Control rats $(n=4)$ were handled in the same way and placed into the self-administration chambers for a number of days matched to each self-administration rat.

\section{FosB-like immunohistochemistry}

Twenty-four hours following the final self-administration session, rats were deeply anesthetised with sodium pentobarbital (100 mg/kg, i.p.) and transcardially perfused with 150 $\mathrm{mL}$ of $0.9 \% \mathrm{NaCl}$ solution containing heparin $(5 \mathrm{IU} / \mathrm{mL})$ followed by $350 \mathrm{~mL}$ of $4 \%$ paraformaldehyde (PFA) solution in $0.1 \mathrm{M}$ potassium phosphate buffer ( $\mathrm{PB}$; $\mathrm{pH}$ 7.4). Perfusions were conducted using a perfusion pump (EYLA micro tube pump MP-3, Tokyo Rikakikai Co. Ltd, Tokyo, Japan) at a flow rate of $\sim 14 \mathrm{~mL} / \mathrm{min}$. Brains were then rapidly removed and placed in 4\% PFA fixative overnight before being placed in a cryoprotective PB solution containing $0.9 \% \mathrm{NaCl}$ (phosphate-buffered saline [PBS]) and 30\% saccharose for 2-3 days. Brains were then frozen in isopentane at $-30^{\circ} \mathrm{C}$, which was gradually cooled to $-50^{\circ} \mathrm{C}$ over $4 \mathrm{~min}$, before being stored at $-80^{\circ} \mathrm{C}$ until sectioning. Coronal sections $(30 \mu \mathrm{m})$ were cut 
along the entire brain using a cryostat (CM3050S, Leica Biosystems), which were then immersed in PBS containing $0.1 \%$ sodium azide and stored at $4{ }^{\circ} \mathrm{C}$.

A one in six series of sections was processed to reveal neurons expressing FosB/ $\Delta$ FosB. Free-floating sections were washed three times in PBS (for 5 min each) containing $0.3 \%$ Triton X-100 (PBST) before being incubated for 10 min with $3 \% \mathrm{H}_{2} \mathrm{O}_{2}$ and washed three times in PBST again. Sections were then incubated for $60 \mathrm{~min}$ with PBST containing $1 \%$ bovine serum albumin, washed three times in PBST, and then incubated overnight with the primary rabbit antibody (Fos B 102, sc-48, Santa Cruz) diluted 1/2000 in PBST. This antibody recognises both FosB and all isoforms of $\Delta \mathrm{FosB}$, but not other Fos family proteins (Perrotti et al., 2005, 2004, 2008). After three washes in PBST, sections were incubated for 120 min with the secondary, biotinylated goat anti-rabbit antibody (BA-1000, Vector Laboratories) diluted 1/1000 in PBST. After another three washes in PBST, sections were then incubated for $60 \mathrm{~min}$ with preformed avidin-biotin complex (Vectastain Elite Kit, PK-6200; Vector Laboratories) diluted 1/1000 in PBST and washed three times in PBST again. Visualisation of bound peroxidase was achieved by reaction in a solution of $50 \mathrm{mM}$ Tris- $\mathrm{HCl}$ buffer containing $0.02 \%$ diaminobenzidine, $0.8 \% \mathrm{NiCl}_{2}$, and $0.003 \% \mathrm{H}_{2} \mathrm{O}_{2}$, to yield a blue-black precipitate. The reaction was stopped by two washes in PBST after $\sim 10 \mathrm{~min}$. Sections were then mounted on gelatin-coated slides, stained with $1 \%$ neutral red, and cover-slipped with DPX mounting medium. A control was run without the primary antibody to ensure the absence of nonspecific immunostaining in the tissue.

Sections were analysed using an Olympus BX-51 light microscope, Lumenera Lt665R camera, and MBF Neurolucida ${ }^{\circledR}$ software. Coded slides were used to blind the observer to the group treatment, with the code only broken at the completion of the experiment. For each brain region, bilateral templates (unilateral for the raphe) were made according to the approximate shape and size of the region (Figure 2.1). All cells within the templates showing positive FosBlike staining were manually counted using Neurolucidia from four coronal sections per region (three for the VTA), per rat, each $180 \mu \mathrm{m}$ apart. Data were averaged across hemispheres and sequential coronal sections for each rat. Final data were expressed as the mean number of FosB $/ \Delta$ FosB labelled cells $/ \mathrm{mm}^{2}$ in each brain region for both the control and MDMA selfadministration conditions. 

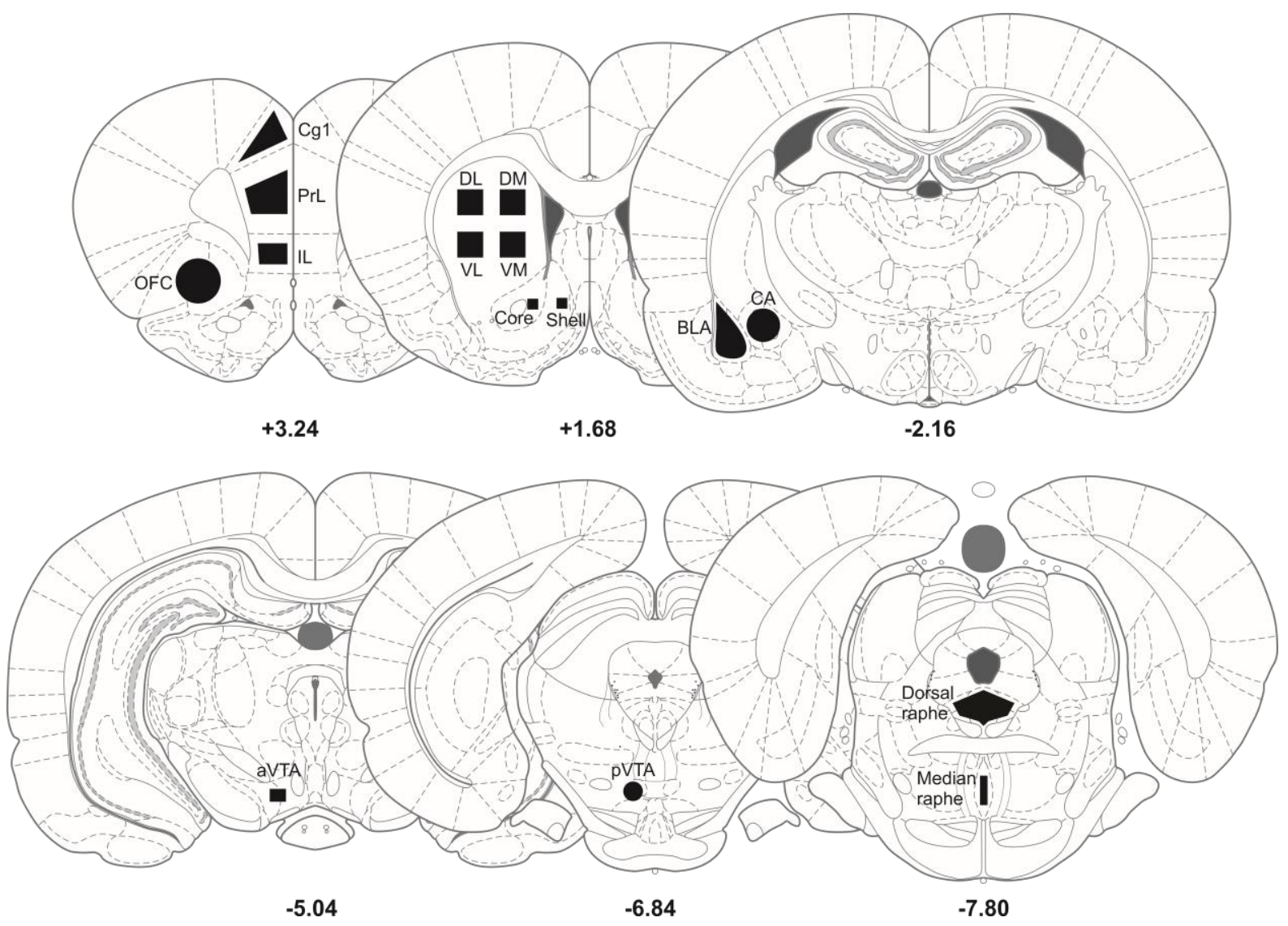

Figure 2.1. Schematic representation (adapted from Paxinos \& Watson, 2005) of the regional templates used for quantification of $\mathrm{FosB} / \Delta \mathrm{FosB}$ immunoreactivity. For clarity reasons, each template is represented only at one bregma level (displayed in mm below each section) on one hemisphere. OFC, orbitofrontal cortex; Cg1, Anterior cingulate; PrL, prelimbic cortex; IL, infralimbic cortex; DL, dorsolateral caudate-putamen; DM, dorsomedial caudate-putamen; VL, ventrolateral caudate-putamen; VM, ventromedial caudate-putamen; core, nucleus accumbens core; shell, nucleus accumbens shell; BLA, basolateral amygdala, CA, central nucleus of the amygdala; aVTA, anterior ventral tegmental area; pVTA, posterior tail of the ventral tegmental area; dorsal raphe; median raphe. 


\section{Statistical analysis}

A one-way repeated-measures analysis of variance (ANOVA) was used to analyse the change in mean MDMA-intake (mg/kg) during the first 29-days of self-administration. Independent samples $t$-tests were used to compare the densities of FosB/ $\Delta$ FosB labelled cells in tissue from each brain region from the control and MDMA self-administration groups. Because there were 14 comparisons made here, the $p$-values from each $t$-test were adjusted using the Hochberg-Bonferroni correction in order to control the false discovery rate (Hochberg, 1988). The Hochberg-Bonferroni method is more powerful than the standard Bonferroni procedure and is, thus, a more appropriate correction to use when a larger number of means are being tested. The level of significance for all tests was $p<.05$. All analyses were conducted using the Statistical Package for Social Sciences (SPSS, v25, IBM).

\section{Results}

Figure 2.2 displays the mean total MDMA intake (mg/kg) over 29 daily sessions of 6hour self-administration. MDMA intake changed significantly as a function of session, $F(28$, $84)=11.14, p<.001, \eta_{\mathrm{p}}^{2}=.788$, and a significant linear contrast indicated an escalation of MDMA intake over the 29-day period, $F(1,3)=76.47, p=.003, \eta_{\mathrm{p}}^{2}=.962$. Inactive lever responding and responding produced by control rats remained low as previously shown (Schenk et al., 2012), with preference for the active lever averaging above $80 \%$.

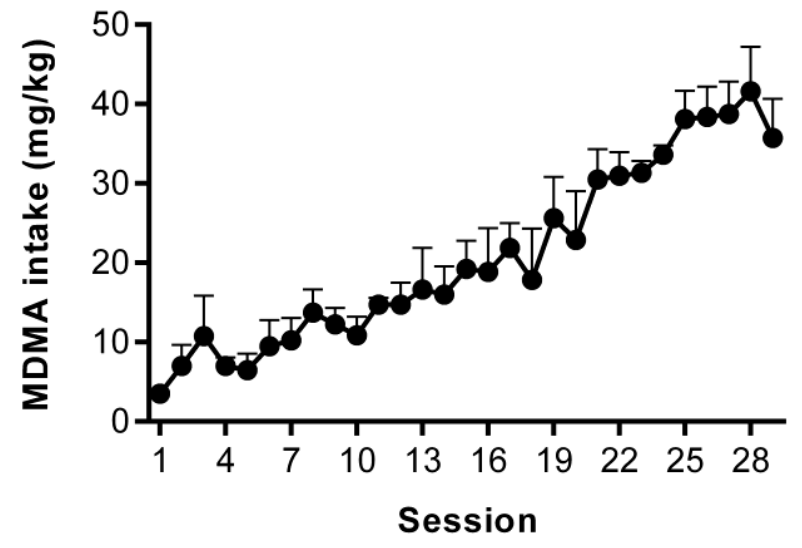

Figure 2.2. Mean MDMA intake (mg/kg) as a function of daily 6-hour self-administration session $(n=4)$. Error bars represent standard error of the mean. 
Figures $2.3-2.8$ shows $\mathrm{Fos} B / \Delta \mathrm{FosB}$ immunoreactivity in various brain regions/subregions as a function of self-administration condition. For each figure, (A) displays conventional light microscopy images of coronal sections of rat brain with the regional templates used for quantification overlaid. (B) Displays higher magnification images of the shaded area presented in A from representative rats in the control and MDMA selfadministration conditions. (C) Displays the mean densities of FosB/ $\Delta$ FosB labelled cells as a function of region and self-administration condition. A summary of the statistical analyses is shown in Table 2.1.

In the ventral striatum/NAcc, MDMA self-administration significantly increased densities of FosB/ $\mathrm{F}$ FosB labelled cells in the core, but differences in the shell were not significant after correction for multiple comparisons (Figure 2.3; Table 2.1). In the dorsal striatum/C-P (Figure 2.4; Table 2.1), MDMA self-administration significantly increased densities of $\operatorname{FosB} / \Delta$ FosB labelled cells in the dorsomedial and ventromedial regions, but not within the dorsolateral or ventrolateral regions. In the PFC (Figure 2.5; Table 2.1) and amygdala (Figure 2.6; Table 2.1), MDMA self-administration significantly increased densities of FosB/ $\Delta$ FosB labelled cells within all subregions. In the raphe, (Figure 2.7; Table 2.1), no FosB $/ \Delta$ FosB labelled cells were detected in the median raphe for either group while significantly greater densities of FosB/ $\Delta$ FosB labelled cells were found in the dorsal raphe of MDMA self-administering rats. In the VTA (Figure 2.8; Table 2.1), no FosB/ $\Delta$ FosB labelled cells were detected in the anterior region for either group while greater densities of FosB/ $\Delta$ FosB labelled cells were observed in the posterior tail of the VTA of MDMA selfadministering rats, but this difference was not significant after correction for multiple comparisons. 

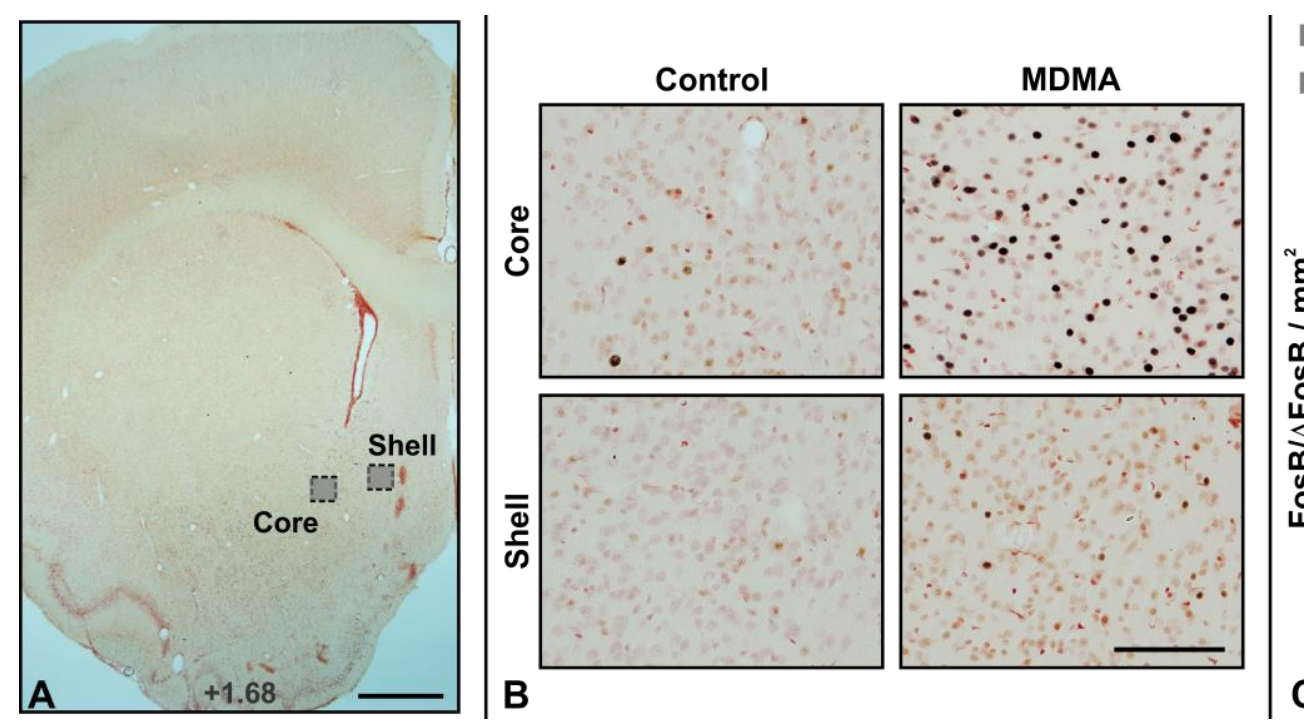

Control $(n=4)$ $\operatorname{MDMA}(n=4)$

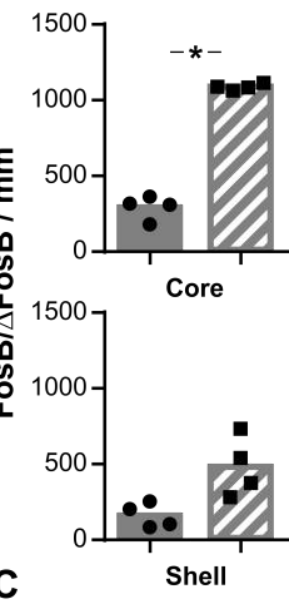

Figure 2.3. Effect of MDMA self-administration on FosB/AFosB immunoreactivity in the ventral striatum/nucleus accumbens (NAcc). (A) Conventional light microscopy image of a coronal section of rat brain with the regional templates used for quantification overlaid. For clarity reasons, each template is represented only at one bregma level $(+1.68 \mathrm{~mm})$, on one hemisphere. Scale bar is $1 \mathrm{~mm}$. (B) Higher magnification images of the shaded areas in A displaying $\mathrm{FosB} / \Delta \mathrm{FosB}$ immunoreactivity in each subregion for representative rats in the control and MDMA self-administration conditions. Scale bar is $100 \mu \mathrm{m}$. (C) Mean FosB/ $\Delta$ FosB positive cells $/ \mathrm{mm}^{2}$ in each subregion for control versus MDMA selfadministering rats. Symbols represent individual subject data. Core, NAcc core; shell, NAcc shell. $* p<.05$ compared to control. 


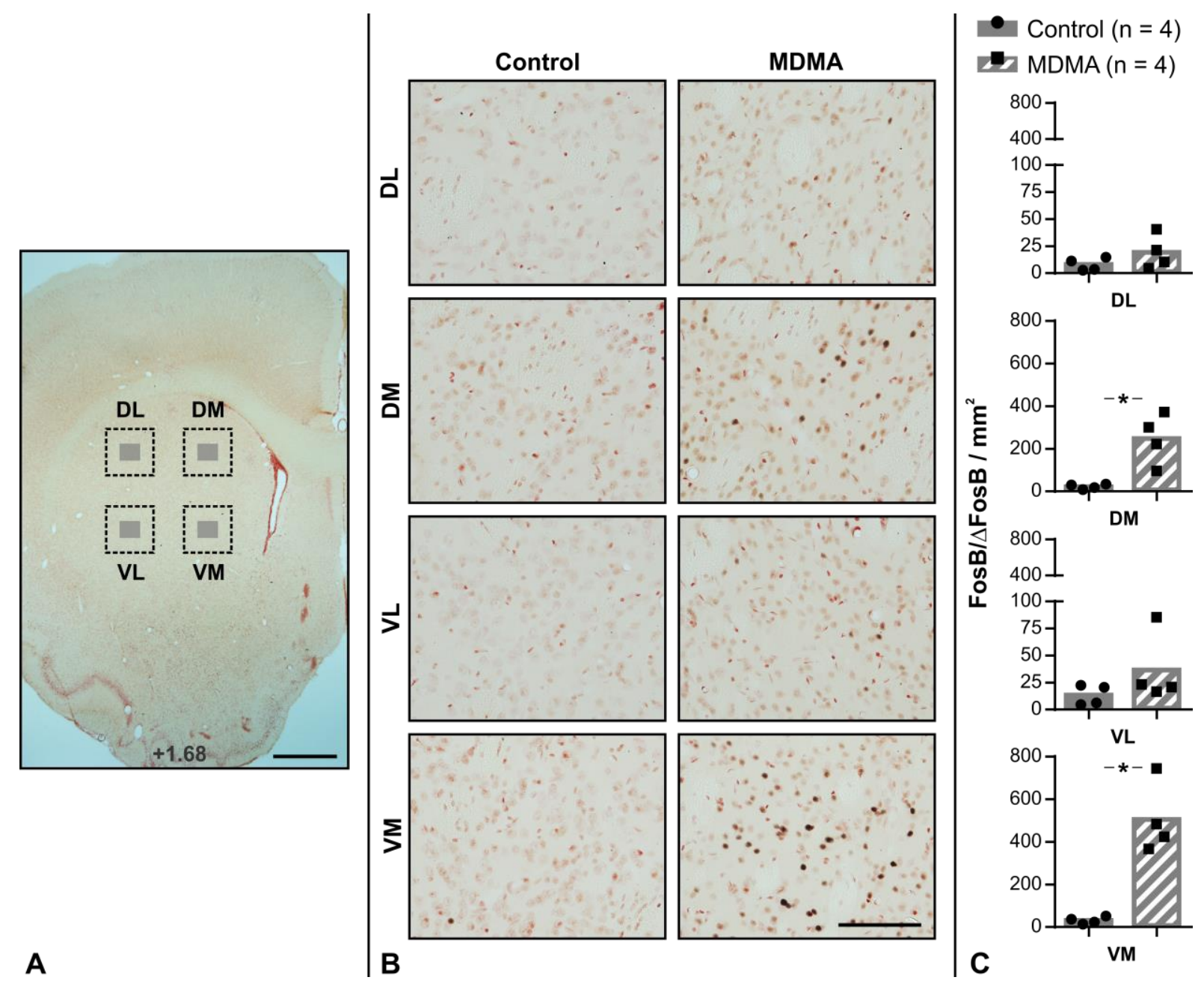

Figure 2.4. Effect of MDMA self-administration on FosB/AFosB immunoreactivity in the dorsal striatum/caudate-putamen (C-P). (A) Conventional light microscopy image of a coronal section of rat brain with the regional templates used for quantification overlaid. For clarity reasons, each template is represented only at one bregma level $(+1.68 \mathrm{~mm})$, on one hemisphere. Scale bar is $1 \mathrm{~mm}$. (B) Higher magnification images of the shaded areas in A displaying FosB/ $\Delta$ FosB immunoreactivity in each subregion for representative rats in the control and MDMA self-administration conditions. Scale bar is $100 \mu \mathrm{m}$. (C) Mean FosB/ $\mathrm{F}$ FosB positive cells $/ \mathrm{mm}^{2}$ in each subregion for control versus MDMA self-administering rats. Symbols represent individual subject data. DL, dorsolateral C-P; DM, dorsomedial C-P; VL, ventrolateral C-P; VM, ventromedial C-P. ${ }^{*} p<.05$ compared to control. 


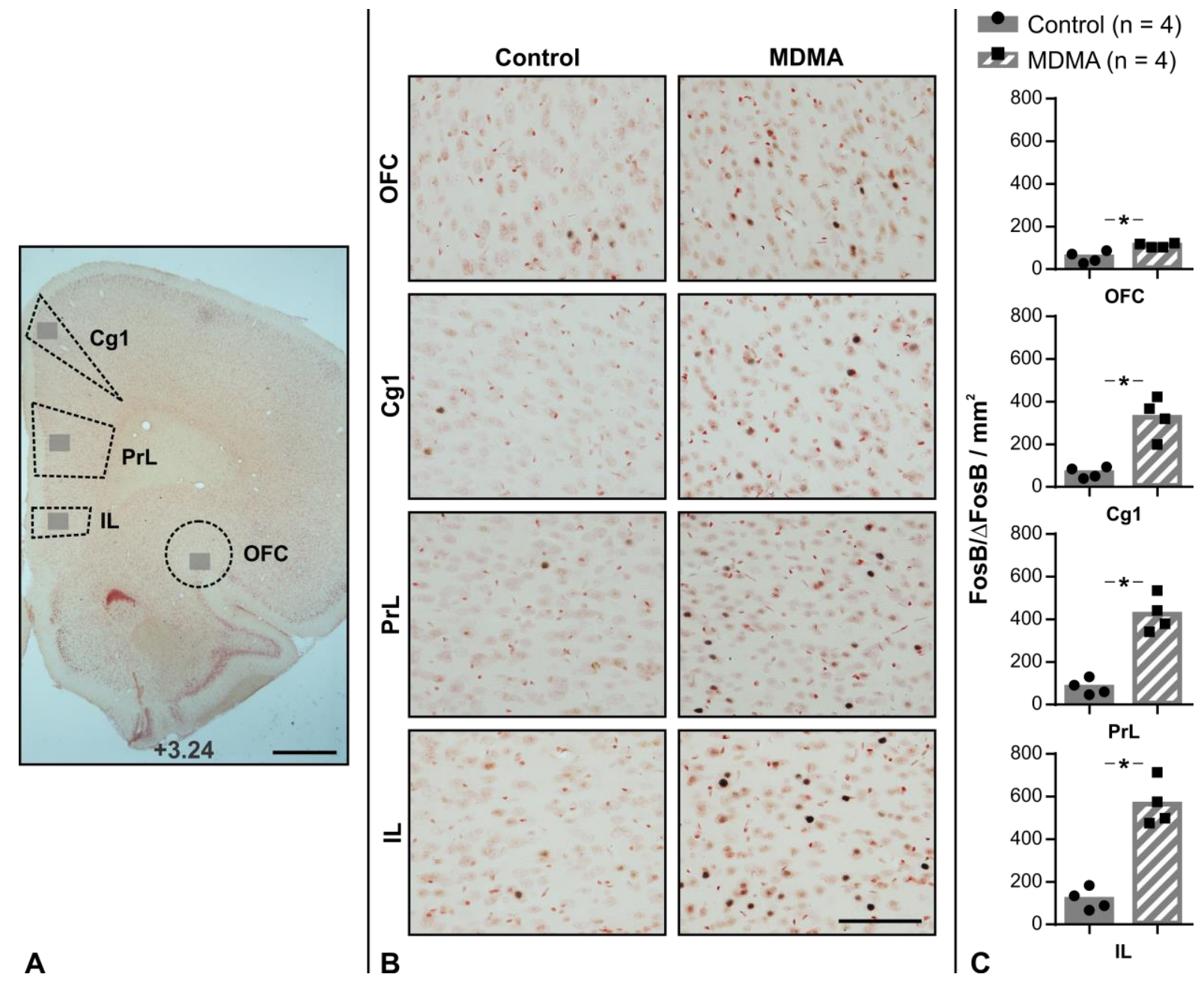

Figure 2.5. Effect of MDMA self-administration on FosB/AFosB immunoreactivity in the prefrontal cortex. (A) Conventional light microscopy image of a coronal section of rat brain with the regional templates used for quantification overlaid. For clarity reasons, each template is represented only at one bregma level $(+3.24 \mathrm{~mm})$, on one hemisphere. Scale bar is $1 \mathrm{~mm}$. (B) Higher magnification images of the shaded areas in $\mathrm{A}$ displaying FosB/ $\Delta$ FosB immunoreactivity in each subregion for representative rats in the control and MDMA selfadministration conditions. Scale bar is $100 \mu \mathrm{m}$. (C) Mean FosB $/ \Delta$ FosB positive cells $/ \mathrm{mm}^{2}$ in each subregion for control versus MDMA self-administering rats. Symbols represent individual subject data. OFC, orbitofrontal cortex; $\mathrm{Cg} 1$, anterior cingulate; PrL, prelimbic cortex; IL, infralimbic cortex. $* p<.05$ compared to control. 

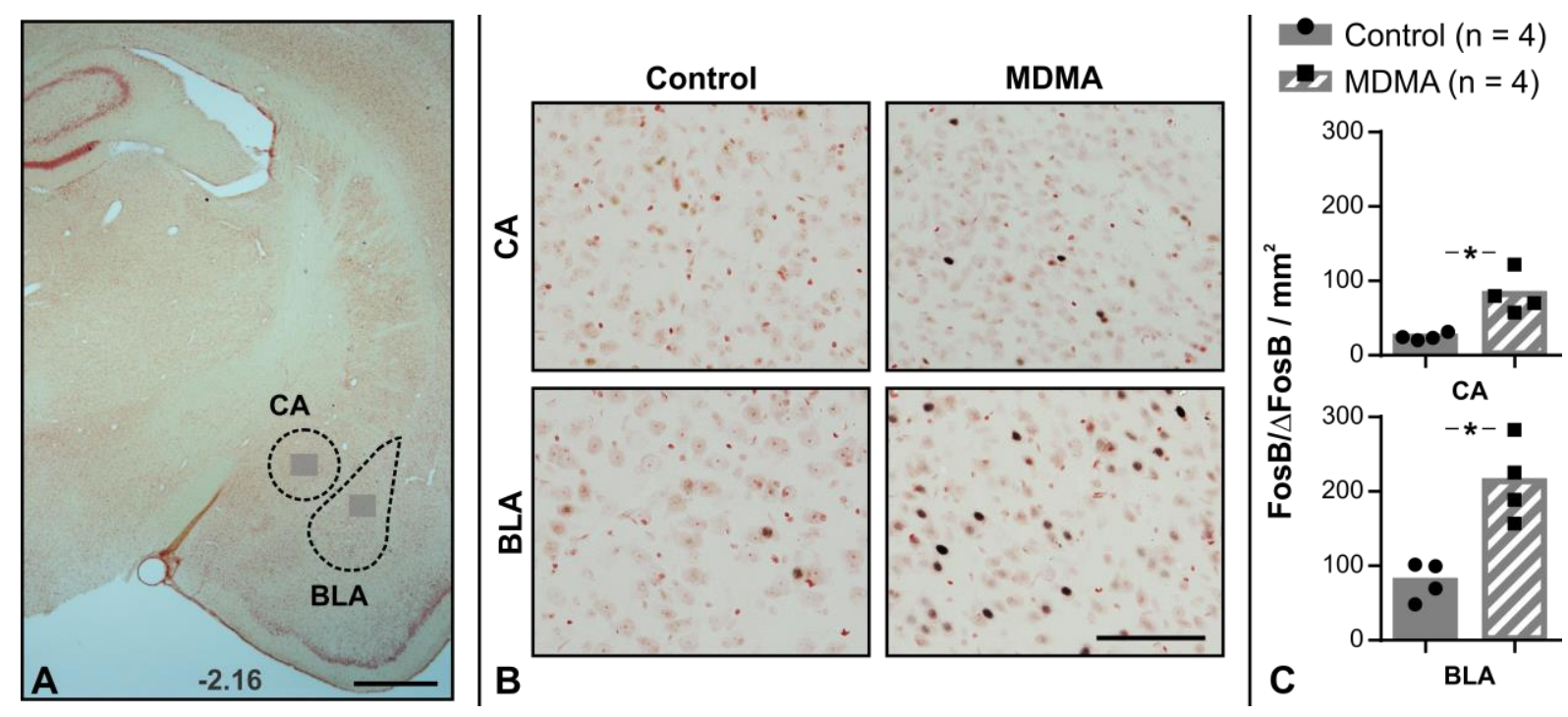

Figure 2.6. Effect of MDMA self-administration on FosB/ $\Delta$ FosB immunoreactivity in the amygdala. (A) Conventional light microscopy image of a coronal section of rat brain with the regional templates used for quantification overlaid. For clarity reasons, each template is represented only at one bregma level $(-2.16 \mathrm{~mm})$, on one hemisphere. Scale bar is $1 \mathrm{~mm}$. (B) Higher magnification images of the shaded areas in $\mathrm{A}$ displaying $\mathrm{FosB} / \Delta \mathrm{FosB}$ immunoreactivity in each subregion for representative rats in the control and MDMA selfadministration conditions. Scale bar is $100 \mu \mathrm{m}$. (C) Mean FosB $/ \Delta$ FosB positive cells $/ \mathrm{mm}^{2}$ in each subregion for control versus MDMA self-administering rats. Symbols represent individual subject data. CA, central nucleus of the amygdala; BLA, basolateral amygdala. * $p$ $<.05$ compared to control. 

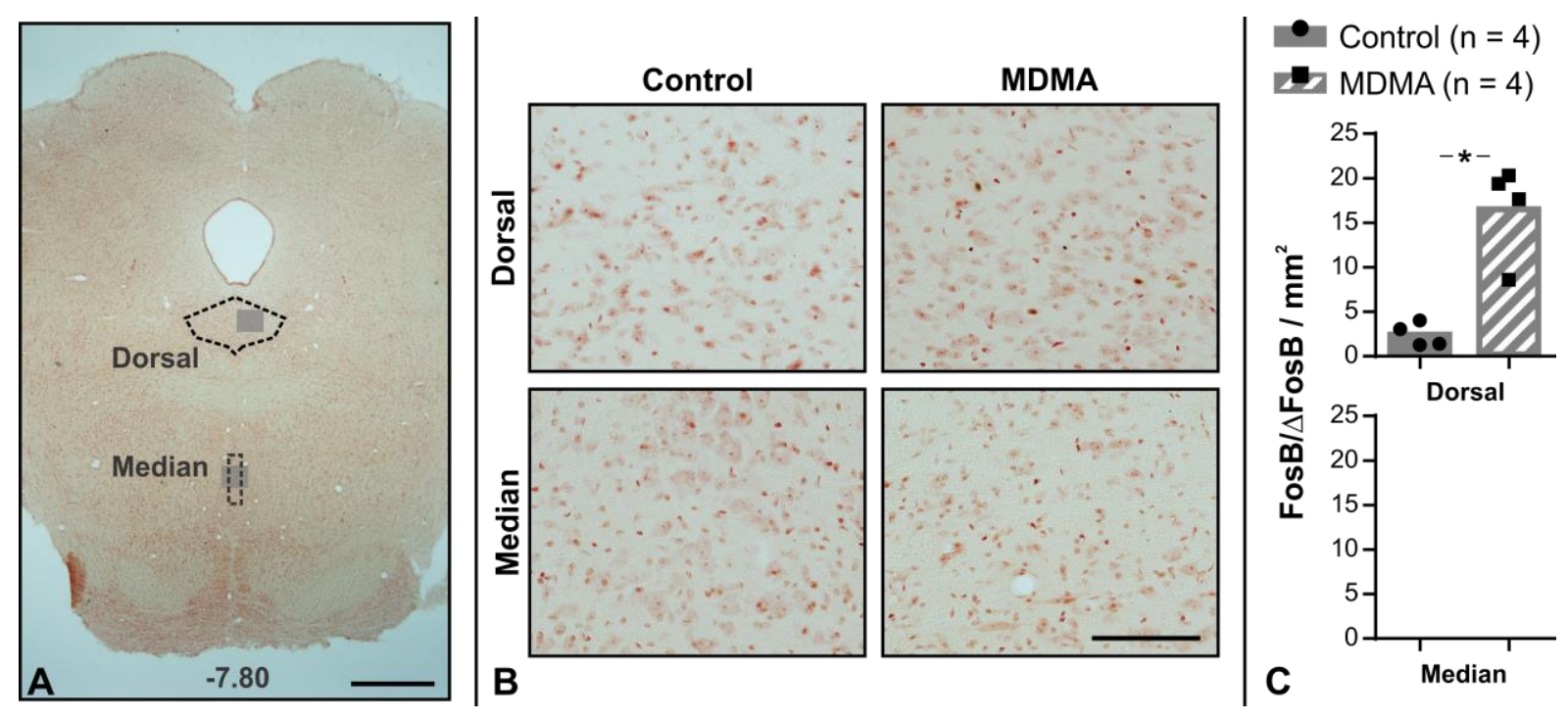

Figure 2.7. Effect of MDMA self-administration on FosB/AFosB immunoreactivity in the dorsal and median raphe. (A) Conventional light microscopy image of a coronal section of rat brain with the regional templates used for quantification overlaid. For clarity reasons, each template is represented only at one bregma level $(-7.80 \mathrm{~mm})$. Scale bar is $1 \mathrm{~mm}$. (B) Higher magnification images of the shaded areas in A displaying FosB/ $\Delta$ FosB immunoreactivity in each subregion for representative rats in the control and MDMA self-administration conditions. Scale bar is $100 \mu \mathrm{m}$. (C) Mean FosB/ $\Delta$ FosB positive cells $/ \mathrm{mm}^{2}$ in each subregion for control versus MDMA self-administering rats. Symbols represent individual subject data. ${ }^{*} p<.05$ compared to control. 

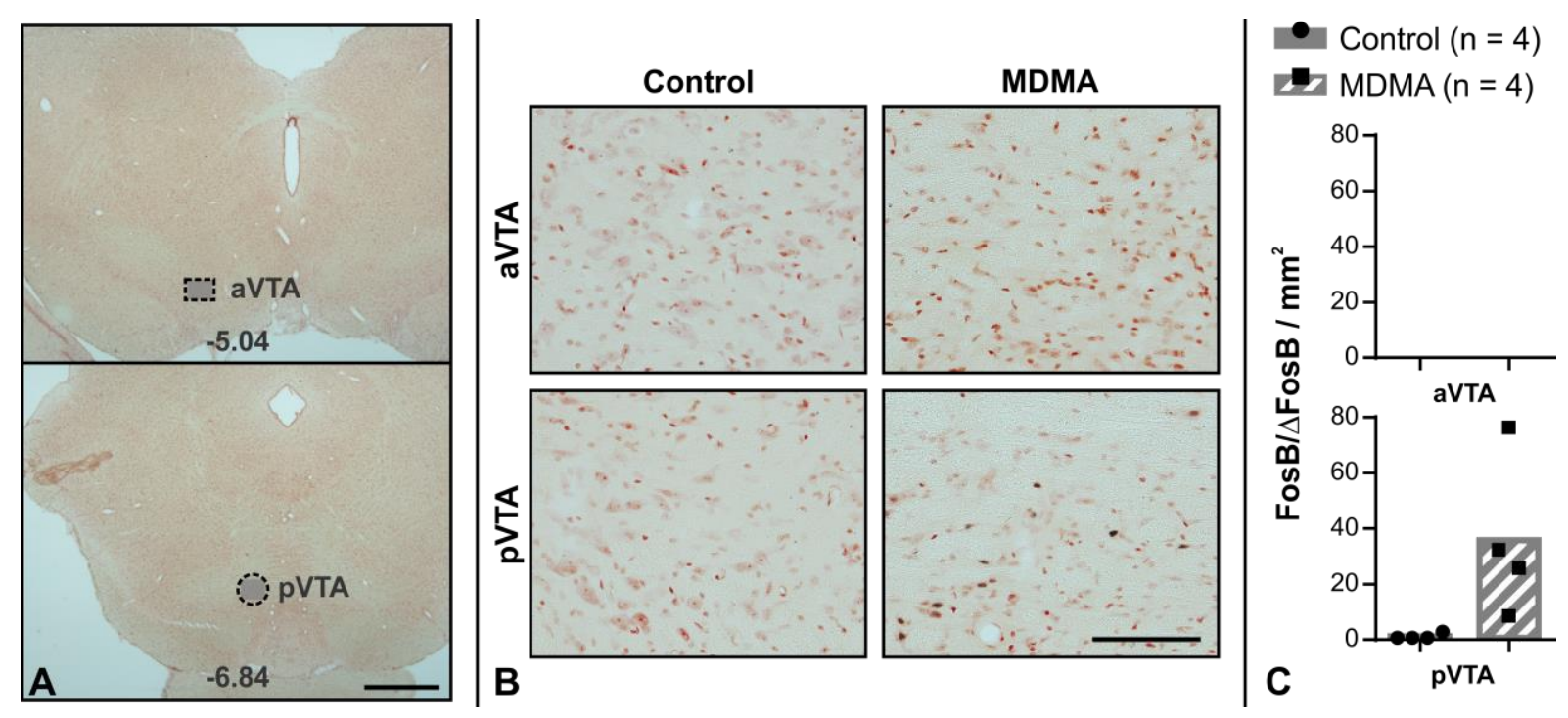

Figure 2.8. Effect of MDMA self-administration on FosB/ $\Delta$ FosB immunoreactivity in the ventral tegmental area (VTA). (A) Conventional light microscopy image of a coronal section of rat brain with the regional templates used for quantification overlaid. For clarity reasons, each template is represented only at one bregma level per region $(-5.04$ or $-6.84 \mathrm{~mm})$, on one hemisphere. Scale bar is $1 \mathrm{~mm}$. (B) Higher magnification images of the shaded areas in A displaying $\mathrm{FosB} / \Delta \mathrm{FosB}$ immunoreactivity in each subregion for representative rats in the control and MDMA self-administration conditions. Scale bar is $100 \mu \mathrm{m}$. (C) Mean FosB/ $\Delta$ FosB positive cells $/ \mathrm{mm}^{2}$ in each subregion for control versus MDMA selfadministering rats. Symbols represent individual subject data. aVTA, anterior VTA; pVTA, posterior tail of the VTA. 
Table 2.1.

Summary of test statistics

\begin{tabular}{lll}
\hline Region / Subregion & $t$-test, Cohen's $d$ & $\begin{array}{c}\text { Corrected } \\
p \text {-value }\end{array}$ \\
\hline Prefrontal cortex & & \\
Orbitofrontal cortex & $t(6)=3.85, p=.008, d=2.72$. & $p=.045^{*}$ \\
Anterior cingulate & $t(6)=5.29, p=.002, d=3.74$. & $p=.019^{*}$ \\
Prelimbic cortex & $t(6)=7.43, p<.001, d=5.25$. & $p=.004^{*}$ \\
Infralimbic cortex & $t(6)=7.51, p<.001, d=5.31$. & $p=.004^{*}$
\end{tabular}

Dorsal Striatum

Dorsolateral caudate-putamen

$t(6)=1.33, p=.231, d=0.94 . \quad p=.231$

Dorsomedial caudate-putamen

$t(6)=3.79, p=.009, d=2.68 . \quad p=.045^{*}$

Ventrolateral caudate-putamen

$t(6)=1.36, p=.224, d=0.96 . \quad p=.231$

Ventromedial caudate-putamen

$t(6)=5.64, p=.001, d=3.99 . \quad p=.015^{*}$

Ventral Striatum

Nucleus accumbens core

$t(6)=19.48, p<.001, d=13.78 . \quad p<.001^{*}$

Nucleus accumbens shell

$t(6)=3.01, p=.024, d=2.13 . \quad p=.094$

Amygdala

Central amygdala

$t(6)=4.07 p=.007, d=2.88 . \quad p=.048^{*}$

Basolateral amygdala

$t(6)=4.47, p=.004, d=3.16 . \quad p=.034^{*}$

Ventral Tegmental Area

Anterior

N/A

Posterior

$t(6)=2.40, p=.038, d=1.87 \quad p=.115$

Raphe

Dorsal

$t(6)=5.08, p=.002, d=0.73$

$p=.020^{*}$

Median

N/A

${ }^{\#}$ Hochberg-Bonferroni correction (Hochberg, 1988), * $p<.05$. 


\section{Discussion}

The transcription factor, $\Delta \mathrm{FosB}$, accumulates within the striatum and some other brain regions following repeated drug exposure (Brenhouse \& Stellar, 2006; Conversi et al., 2007; Cornish et al., 2012; De Pauli et al., 2014; Kaplan et al., 2011; Larson et al., 2010; McDaid et al., 2006; Perrotti et al., 2005, 2008; Pich et al., 1997; Winstanley et al., 2007). $\Delta$ FosB has been identified as an important regulator of long-term neuroplasticity within these brain regions and has, therefore, been suggested to be relevant to addiction (Frankowska, Dudek, \& Siwek, 2016; Mews et al., 2019; Nestler, 2008). In this study, the impact of chronic MDMA exposure on $\Delta$ FosB accumulation in numerous brain areas was demonstrated for the first time.

IHC was used to map the accumulation of $F o s B / \Delta F$ osB in 16 different brain regions that have been implicated in various aspects of addiction. This was carried out following a minimum of 29 long-access ( 6 hour) daily MDMA self-administration sessions that resulted in an escalation of drug intake and that has previously been shown to produce extensive drugseeking behaviour (Schenk et al., 2016, 2011), two measures that have been suggested to be consistent with the development of drug addiction (Ahmed, 2011; Edwards \& Koob, 2013).

Significant region-specific increases in the accumulation of $\mathrm{FosB} / \Delta \mathrm{FosB}$ as a function of MDMA self-administration were observed. The pattern of FosB/ $\Delta$ FosB expression was largely similar to those seen following repeated experimenter- or self-administered exposure to other drugs of abuse. The antibody used in this study labels both FosB and all isoforms of $\Delta \mathrm{FosB}$, although it is likely that these results primarily reflect stable isoforms of $\Delta \mathrm{FosB}$. This is because these results were collected 24 hours following the final self-administration session and during these 24 hours, all non-stable forms of FosB would have degraded, leaving only $\Delta \mathrm{FosB}$, as has been previously demonstrated using double labelling procedures (Perrotti et al., 2005, 2004, 2008).

The most pronounced induction of $\Delta$ FosB across all the regions was observed within the striatal complex, particularly within the ventral striatum/NAcc. This was not surprising as virtually all drugs of abuse tested have been shown to induce $\Delta \mathrm{FosB}$ expression in the NAcc following repeated exposure (Nestler, 2008; Perrotti et al., 2008). In the current study, $\Delta$ FosB expression was higher in both the NAcc core and shell following MDMA self-administration, but the difference in the shell was not significant following statistical correction for multiple comparisons.

An important role of accumbal $\Delta$ FosB in the reinforcing effects of drugs has been demonstrated by studies that have manipulated $\Delta \mathrm{FosB}$ expression. Overexpression of $\Delta \mathrm{FosB}$ 
in the NAcc and surrounding striatal areas increased both cocaine- (Kelz et al., 1999) and morphine- (Zachariou et al., 2006) produced CPP. In contrast, local overexpression of a dominant-negative antagonist of $\Delta$ FosB-mediated transcription, $\Delta \mathrm{c}$-Jun, reduced cocaine CPP (Peakman et al., 2003). Importantly, $\Delta$ FosB overexpression also facilitated cocaine selfadministration (Colby et al., 2003).

Pronounced increases in $\Delta \mathrm{FosB}$ expression were also observed in subdivisions of the dorsal striatum/C-P following MDMA self-administration. There is considerable functional heterogeneity between the lateral and medial C-P. The former is crucial for the development of stimulus-response habits while the latter mediates action-outcome or goal-directed behaviour (Everitt \& Robbins, 2016; H.-J. Kim, Lee, Yun, \& Kim, 2017; Yin \& Knowlton, 2006). This is consistent with differences in the anatomical connectivity of the medial and lateral C-P; the lateral portion receives excitatory input from the sensorimotor cortices whereas the medial portion receives excitatory input more so from the medial PFC (Crittenden \& Graybiel, 2011; Voorn et al., 2004). Therefore, in the current study, the analysis of $\Delta$ FosB accumulation in the C-P was divided into the dorsolateral, dorsomedial, ventrolateral, and ventromedial portions.

Significant increases in $\triangle$ FosB accumulation following MDMA self-administration were only found in the two medial portions of the C-P, with the most pronounced increase in the ventromedial portion. A similar pattern of results has also been observed following repeated amphetamine exposure (Conversi et al., 2007) and following the self-administration of methamphetamine (Cornish et al., 2012), cocaine (Pich et al., 1997), and nicotine (Pich et al., 1997). These results suggest that repeated drug exposure results in the accumulation of $\Delta \mathrm{FosB}$ in regions of the C-P involved in the acquisition and expression of goal-directed actions more so than regions involved in habitual, stimulus-driven instrumental behaviour.

MDMA self-administration produced significant increases in $\triangle F o s B$ expression in several brain regions outside of the striatum, most notably within the medial PFC. Significant increases in $\triangle \mathrm{FosB}$ expression were observed in the anterior cingulate, prelimbic, and infralimbic cortices, with the increase being more pronounced in ventral regions. Similar results have been observed across the medial PFC following repeated exposure to amphetamine (Conversi et al., 2007) and morphine (Kaplan et al., 2011), as well as following the selfadministration of cocaine (Pich et al., 1997; Winstanley et al., 2007) and nicotine (Pich et al., 1997). Smaller, yet significant increases in $\triangle F$ osB expression were also found in the OFC akin 
to those found following self-administration of methamphetamine (Cornish et al., 2012) and cocaine (Winstanley et al., 2007).

The PFC is involved in several cognitive and behavioural processes that become disrupted following repeated drug exposure (Goldstein \& Volkow, 2011). This has been suggested to play a major role in the development and maintenance of drug addiction (Goldstein \& Volkow, 2011). Drug-induced accumulation of $\triangle F$ osB within the PFC might contribute to the dysregulated functioning of the PFC since local overexpression of $\triangle F$ osB in the OFC mimicked the effect of repeated cocaine exposure on rodent tests of attention and decision making (Winstanley et al., 2007). In contrast, overexpression of $\Delta \mathrm{JunD}$, which antagonises $\Delta$ FosB-mediated transcription, prevented the effects of repeated cocaine exposure on these tests (Winstanley et al., 2007).

Significant increases in $\triangle \mathrm{FosB}$ were also observed in both the CeA and BLA following MDMA self-administration. The increase was most pronounced in the BLA. Previous studies have found similar increases in $\Delta \mathrm{FosB}$ expression following methamphetamine selfadministration (Cornish et al., 2012), and following repeated exposure to morphine, cocaine, and nicotine (Kaplan et al., 2011; Perrotti et al., 2008).

The amygdala plays crucial roles in several aspects of addiction; the BLA is particularly critical for conditioned-reinforcement and cue-induced drug-seeking while the CeA is particularly important for stress-induced drug-seeking and emotional regulation (Everitt \& Robbins, 2016; Koob \& Volkow, 2016). Like other drugs of abuse, extinguished MDMAseeking behaviour can be reinstated by a drug prime (Schenk et al., 2011), a stress inducer (Ball et al., 2015), and by the presentation of conditioned cues (Ball et al., 2007; Schenk et al., 2011). Also like other drugs, conditioned cues and conditioned reinforcement play crucial roles in MDMA self-administration (Daniela et al., 2006). $\Delta$ FosB-mediated transcription within the $\mathrm{CeA}$ and BLA might affect these processes.

Unlike other psychostimulant drugs of abuse that primarily impact the DAergic system, MDMA also dramatically increases synaptic 5-HT. DAergic and 5-HTergic cell bodies originate from the VTA and raphe, respectively, and innervate many of the previously discussed brain regions. For this reason, $\triangle F$ FosB accumulation in the anterior/posterior VTA and the dorsal/median raphe was also measured.

No $\Delta$ FosB labelled cells were detected in the median raphe in either self-administration condition. In the dorsal raphe, MDMA self-administration resulted in small, but significant increases in $\Delta \mathrm{FosB}$ expression, similar to those produced by chronic methamphetamine self- 
administration (Cornish et al., 2012). Although the dorsal raphe is densely populated with 5HTergic neuron cell bodies, further work would be needed to determine if these are indeed 5HT neurons expressing $\triangle$ FosB. $\triangle$ FosB is typically only expressed within GABAergic neurons (Nestler, 2008), which are also present in the dorsal raphe (M. F. Belin et al., 1979, 1983; Charara \& Parent, 1998; Nanopoulos, Belin, Maitre, Vincendon, \& Pujol, 1982). Given the low density of dorsal raphe $\Delta$ FosB detected in the current study, these may be GABAergic raphe neurons expressing $\Delta$ FosB.

In the anterior VTA, previous studies have failed to find increases in $\triangle \mathrm{FosB}$ expression following repeated exposure to various drugs of abuse (McDaid et al., 2006; Perrotti et al., $2005,2008)$. It was, therefore, not surprising that the current study also failed to detect $\Delta$ FosB within this brain region following MDMA self-administration. In the posterior tail of the VTA, however, MDMA self-administration resulted in increased levels of $\Delta$ FosB that were approaching significance. It might be surprising that a stronger effect was not observed in this region since increased FosB-like expression in the posterior tail of the VTA has been previously observed when measured 3 hours following acute MDMA exposure (Kaufling et al., 2010). This may be due to the quantification of several other non-stable FosB-like proteins in addition to $\Delta \mathrm{FosB}$, however. In the current study, these non-stable isoforms of FosB would have degraded when the rats were perfused 24 hours following drug exposure, leaving only $\Delta$ FosB (Perrotti et al., 2005, 2004, 2008). Exposure to several other psychostimulant drugs has also been shown to induce $\Delta$ FosB expression in the posterior VTA (Cornish et al., 2012; Kaufling et al., 2010; Perrotti et al., 2005, 2008). In one study, posterior VTA $\Delta$ FosB expression was shown to be selective for GABAergic neurons and due to the DAergic action of the drugs since selective 5-HTergic and NAergic acting agents failed to have an effect (Kaufling et al., 2010). It is likely that the $\Delta$ FosB detected in the current study was similarly expressed within GABAergic posterior VTA neurons and due to the DAergic effects of MDMA.

In summary, MDMA self-administration produced an accumulation of $\Delta \mathrm{FosB}$ in several of the same brain regions as repeated exposure to other drugs of abuse. These brains regions are known to play crucial roles in the development and maintenance of drug addiction. Striatal regions that are involved in goal-directed behaviour (NAcc, DMS) showed a particularly pronounced accumulation of $\Delta$ FosB. Brain regions involved in several other important aspects of addiction, such as executive functioning and conditioning also showed increased $\triangle$ FosB expression. These regions included the CeA, BLA, and most major 
subdivisions of the PFC. It has been suggested that MDMA self-administration progresses as a function of neuroadaptive responses in the same brain regions that mediate the progression of self-administration of other drugs of abuse (Schenk, 2011). The present data are consistent with this idea and also suggest that $\Delta \mathrm{FosB}$ accumulation in several brain regions that are relevant to addiction is a common consequence of repeated exposure to drugs of abuse. 
Parts of this chapter have been adapted with licensed permission from work published in Addiction Biology (van de Wetering \& Schenk, 2019).

\section{Introduction}

Repeated intermittent, drug exposure can result in the sensitisation of various drug produced behaviours such as locomotor activity and stereotypy (Pierce \& Kalivas, 1997; Post \& Rose, 1976; T. E. Robinson, 1984; Stewart \& Badiani, 1993). It has been suggested that the development of sensitisation to these behavioural effects of drugs is relevant to addiction since repeated drug exposure under these same conditions can also sensitise the reinforcing effects of drugs, as evidenced by the facilitated acquisition of self-administration, higher breakpoints on PR schedules, and increased CPP (Berridge \& Robinson, 2016; Pierce \& Kalivas, 1997; T. E. Robinson \& Berridge, 1993, 2008; Vanderschuren \& Pierce, 2010; Vezina, 2004). Further, the mechanisms underlying the development and expression of these sensitised responses appear to be similar and involve various neuroadaptations in brain regions such as the VTA, NAcc, dorsal striatum, and PFC (Berridge \& Robinson, 2016; Pierce \& Kalivas, 1997; T. E. Robinson \& Berridge, 1993, 2008; Vanderschuren \& Pierce, 2010; Vezina, 2004).

Like other psychostimulant drugs, acute administration of MDMA produces dosedependent increases in locomotor hyperactivity (Brennan \& Schenk, 2006; L. H. Gold, Koob, \& Geyer, 1988; Spanos \& Yamamoto, 1989). Also like other psychostimulant drugs, sensitisation of the locomotor activating as well as the reinforcing effects of MDMA was produced following repeated MDMA exposure (van de Wetering \& Schenk, 2017). Sensitisation of the locomotor activating effects of MDMA has also been observed following MDMA self-administration (Schenk \& Bradbury, 2015). Relatively little is known about the brain regions involved in the development or expression of these sensitised responses to MDMA, however.

The brain regions in which local MDMA administration result in the development or expression of MDMA behavioural sensitisation has not been previously determined. Indeed, the acute locomotor activating effect of locally administered MDMA has also not been previously determined. These were assessed in the current study. Rats were pretreated with a sensitising regimen of systemic, repeated MDMA exposure and the locomotor activating effect of MDMA administered directly into the dorsomedial (medial C-P), dorsolateral (lateral C-P), 
and ventral (NAcc) striatum was determined. This study was guided by the results of the previous chapter where it was shown that repeated MDMA exposure increased $\triangle$ FosB accumulation within the ventral and dorsomedial, but not the dorsolateral areas of the striatum. Thus, the current research aimed to determine if these region-specific increases in striatal $\Delta$ FosB were relevant to the expression of behavioural sensitisation caused by repeated MDMA exposure.

Previous research has implicated an important role of $\Delta$ FosB in the development and expression of sensitised behavioural responses to other drugs of abuse. Enhanced $\Delta$ FosB levels in the ventromedial C-P and NAcc was associated with the development of methamphetamine behavioural sensitisation (Conversi et al., 2007; McDaid et al., 2006). Opiate-induced behavioural sensitisation was also accompanied by enhanced $\Delta$ FosB levels in the NAcc and CP (Kaplan et al., 2011) while both cocaine- and nicotine-induced behavioural sensitisation were accompanied by enhanced $\Delta$ FosB levels in the NAcc (Brenhouse \& Stellar, 2006; Marttila et al., 2006). Compellingly, experimenter-induced overexpression of $\Delta \mathrm{FosB}$ in the NAcc using transgenic animals also sensitised cocaine-produced locomotor hyperactivity, facilitated the acquisition of cocaine self-administration, increased the motivation to self-administer cocaine on PR schedules, and increased both cocaine- and morphine-produced CPP (Colby et al., 2003; Grueter et al., 2013; Kelz et al., 1999; Zachariou et al., 2006). These findings suggest a causal relationship between striatal $\Delta$ FosB and the development of these sensitised responses. Given the role of striatal $\Delta \mathrm{FosB}$ in the development and expression of behavioural sensitisation to other drugs of abuse, it was predicted that MDMA behavioural sensitisation would be expressed by local administration of MDMA into the same brain regions that also showed enhanced accumulation of $\triangle \mathrm{FosB}$ following repeated MDMA exposure, as demonstrated in Chapter 2.

\section{Methods}

\section{Subjects}

Male Sprague-Dawley rats were bred and housed as described in Chapter 2. All experimental protocols were approved by the Animal Ethics Committee of Victoria University of Wellington.

\section{Surgical procedures}

Pre- and post-operative surgical procedures were carried out as described in Chapter 2. Rats were stereotaxically implanted with stainless-steel, 22-gauge, bi-lateral guide cannula (Plastics One, Roanoke, Va., USA) aimed $1 \mathrm{~mm}$ above the NAcc, medial C-P, or lateral C-P. 
Targeted stereotaxic coordinates were as follows: NAcc: $+2.2 \mathrm{~mm}$ anteroposterior (AP), \pm 1.5 mm mediolateral (ML), -6.0 mm dorsoventral (DV); medial C-P: $+1.8 \mathrm{~mm} \mathrm{AP,} \pm 2.0 \mathrm{~mm} \mathrm{ML}$, -4.2 mm DV; lateral C-P: 1.5 mm AP, $\pm 3.5 \mathrm{~mm} \mathrm{ML,}-4.2 \mathrm{~mm} \mathrm{DV}$; all coordinates relative to bregma/skull with incisor bar $-3.9 \mathrm{~mm}$ from interaural line (Paxinos \& Watson, 2005). Guide cannulae were lowered into position and secured in place with screws and dental acrylic. Following surgery, 28-gauge dummy cannulae (Plastics One, Roanoke, Va., USA) with a 0.5 $\mathrm{mm}$ projection were inserted into the guide cannula to prevent obstruction.

\section{Apparatus}

Locomotor activity was measured in clear plexiglass chambers $(42 \times 42 \times 30 \mathrm{~cm}$; Med Associates Inc., USA; model ENV-515) set in sound-attenuating boxes. Each chamber contained two sets of 16 infrared sensors that were spaced $2.5 \mathrm{~cm}$ apart, which produced a lattice of beams that was $1.7 \mathrm{~cm}$ above the floor of the chamber. The sequential interruption of three beams (the approximate size of the body of the rat) was recorded as one (horizontal) locomotor activity count. Another series of beams spaced $2.5 \mathrm{~cm}$ apart and located $15 \mathrm{~cm}$ above the floor of the chamber was used to measure vertical locomotor activity (i.e. rearing behaviour). Any interruption of at least one of these beams was recorded as one rearing count. A white noise generator was used to mask noise and the chambers were washed with Virkon ${ }^{\mathrm{TM}}$ $\mathrm{S}$ disinfectant after each session. All locomotor experiments were run in a temperaturecontrolled $\left(21^{\circ} \mathrm{C}\right)$ room illuminated by dim red lighting. Locomotor activity was recorded in 5 min bins. Data acquisition was controlled by Activity Monitor software (Med Associates Inc.).

\section{Drugs}

\pm MDMA $\mathrm{HCl}$ (BDG synthesis, New Zealand) was dissolved in a sterile $0.9 \% \mathrm{NaCl}$ solution for i.p. injections, and in aCSF $(137 \mathrm{mM} \mathrm{NaCl}, 2.7 \mathrm{mM} \mathrm{KCl}, 1.2 \mathrm{mM} \mathrm{CaCl}, 0.5 \mathrm{mM}$ $\mathrm{MgCl}_{2}, 8.1 \mathrm{mM} \mathrm{Na}_{2} \mathrm{HPO}_{4}, 1.5 \mathrm{mM} \mathrm{KH} \mathrm{PO}_{4}$ ) for intra-striatal microinjections.

\section{Pretreatment and intra-striatal microinjections}

During each of 5 daily pretreatment sessions, rats were placed into locomotor activity chambers for $30 \mathrm{~min}$ prior to receiving MDMA (0.0 or $10 \mathrm{mg} / \mathrm{kg}$, i.p.) and remained in the locomotor activity chambers for an additional $60 \mathrm{~min}$ thereafter. This pretreatment regimen was used because it has previously been shown to sensitise the locomotor activating effects of MDMA and facilitated the acquisition of MDMA self-administration (van de Wetering \& Schenk, 2017). 
Following two drug-free days, the locomotor activating effect of MDMA administered locally into the NAcc, medial C-P, or lateral C-P was determined. Rats were briefly removed from the chambers and loosely restrained while the dummy cannula was removed, and a 28gauge stainless-steel bi-lateral infusion cannula (Plastics One, Roanoke, Va., USA) with a 1 $\mathrm{mm}$ projection was inserted. Infusion cannulae were connected to $10 \mu \mathrm{L}$ Hamilton syringes housed in a mechanical infusion pump (PHD 2000 infusion, Harvard Apparatus). MDMA (200 $\mu \mathrm{g} / \mu \mathrm{L} /$ side; total volume $1 \mu \mathrm{L} /$ side) was administered over 2 min with infusion cannulae remaining in place for 1 additional minute. Locomotor activity was recorded in 5 min bins for 30 min prior to and 60 min following the MDMA infusion. This dose was chosen based on the results of pilot studies that showed moderate levels of locomotor activity following this dose of MDMA when injected into the striatum (Figure 3.1).

At the completion of the experiment, animals were euthanised by $\mathrm{CO}_{2}$ asphyxiation. Brains were rapidly removed and placed in 4\% PFA fixative overnight before being frozen at $-80^{\circ} \mathrm{C}$ until sectioning. Coronal sections $(100 \mu \mathrm{m})$ were cut along the striatum using a sliding microtome (HM-450 with KS-34 freezing unit, Thermo Scientific). Sections were then mounted on gelatin-coated slides, stained with $1 \%$ neutral red, and cover-slipped with DPX mounting medium. Light microscopy was used to verify the coordinates of the infusion cannula (Figure 3.2). Data from rats with placements outside of the target area were excluded from any analysis (see Figure 3.3 for final sample sizes).

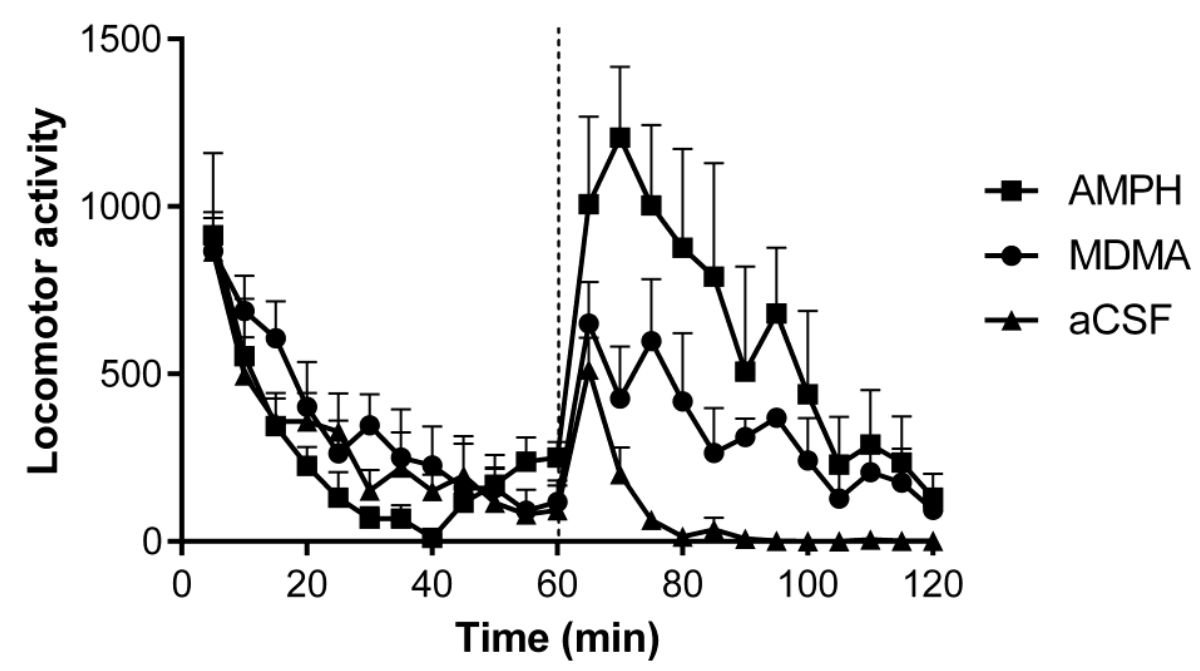

Figure 3.1. Pilot study results showing mean (+ standard error of the mean) locomotor activity as a function of time and bilateral drug administration into the central striatum. Amphetamine (AMPH; $20 \mu \mathrm{g} / 1 \mu \mathrm{L} /$ side), MDMA (200 $\mu \mathrm{g} / 1 \mu \mathrm{L} /$ side), or vehicle (artificial cerebrospinal fluid [aCSF]) was at administered at $60 \min . n=3$ per group. 


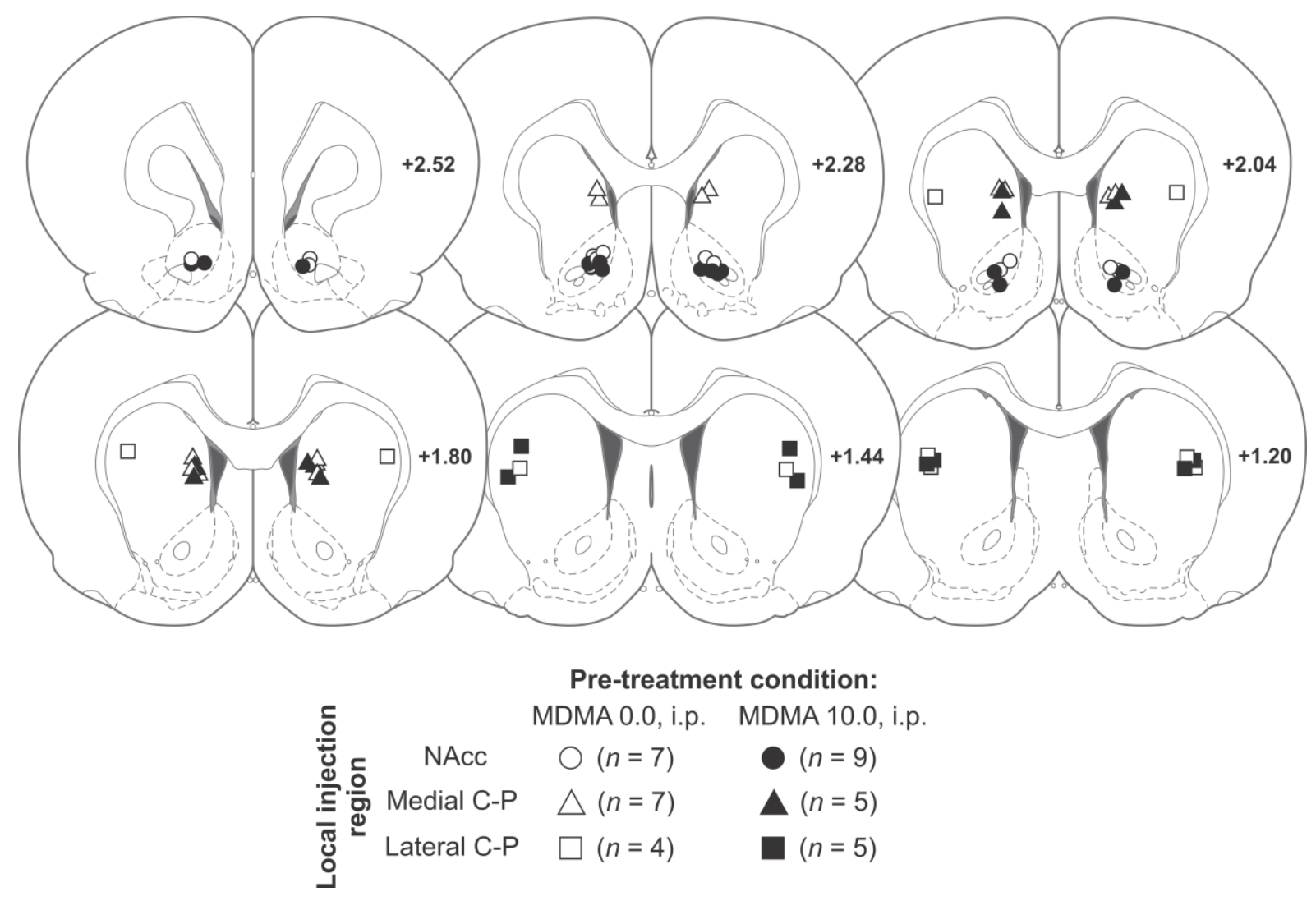

Figure 3.2. Schematic representation (adapted from Paxinos \& Watson, 2005) of the location of the tip of the cannula for local MDMA injections $(200 \mu \mathrm{g} / 1 \mu \mathrm{L} / \mathrm{side})$ into the nucleus accumbens (NAcc; ○, •), medial caudate-putamen (C-P; $\Delta, \boldsymbol{\Delta})$, and lateral C-P $(\square, \mathbf{\square}$, following pretreatment with $0.0(\circ, \Delta, \square)$ or $10.0(\bullet, \boldsymbol{\Delta}, \boldsymbol{\square}) \mathrm{mg} / \mathrm{kg}$ i.p. MDMA. Numbers indicate distance in $\mathrm{mm}$ along the anteroposterior plane from bregma.

\section{Statistical analysis}

Separate 2 (pretreatment: MDMA 0.0 vs 10.0) × 18 (time: -30 - 60 min) mixedmeasures ANOVAs were conducted to analyse locomotor activity and rearing produced by microinjections of MDMA into each brain region. Sphericity could not be assumed for these tests (and follow-up tests), and therefore, Greenhouse-Geisser corrections to degrees of freedom were applied (Greenhouse \& Geisser, 1959). Significant interactions were followed up with simple main effect analyses. A priori pairwise comparisons (simple when following significant simple main effects) were conducted to compare MDMA-produced locomotor activity and rearing at each time point to the final time point of the $30 \mathrm{~min}$ habituation period (i.e. -5 min time-period). Separate independent samples $t$-tests were used to analyse the total locomotor activity and rearing produced during the $60 \mathrm{~min}$ following microinjections of MDMA into each brain region. The level of significance for all tests was $p<.05$. All analyses were conducted using SPSS (v25, IBM). 


\section{Results}

Figure 3.3 and 3.4 displays mean locomotor activity and rearing, respectively, as a function of MDMA pretreatment during the $30 \mathrm{~min}$ prior to and $60 \mathrm{~min}$ following microinjections of MDMA into the NAcc, medial C-P, and lateral C-P. The inserts in each graph display the mean 60 min total of locomotor activity or rearing produced following MDMA microinjections. Both behavioural measures showed similar effects across brain regions and treatment groups. The pre-injection activity profile was mostly comparable for all groups; initial activity counts were high, and activity decreased during over 30 min period. Following MDMA administration, markedly different activity profiles were observed as a function of both pretreatment and brain region.

Microinjections of MDMA into the NAcc produced locomotor hyperactivity for both pretreatment groups, but greater hyperactivity was observed in the MDMA 10.0 group. There was a significant two-way interaction (pretreatment $\times$ time), $F(2.42,33.77)=4.542, p=.013$, $\eta_{\mathrm{p}}{ }^{2}=.245$. Locomotor activity changed significantly as a function of time for both the MDMA $0.0, F(2.43,14.58)=8.68, p=.002, \eta_{\mathrm{p}}{ }^{2}=.591$ and MDMA 10.0, $F(2.12,16.95)=19.83, p<$ $.001, \eta_{\mathrm{p}}^{2}=.713$ pretreatment groups. MDMA-produced locomotor hyperactivity was both of greater magnitude and longer duration for the MDMA 10.0 group (see Figure 3.3, $p<.05$ ). Total MDMA-produced locomotor activity was also significantly greater in the MDMA 10.0 group, $t(14)=3.00, p=.009, d=1.58$.

Microinjections of MDMA into the medial C-P only produced locomotor hyperactivity in rats pretreated with MDMA 10.0. There was a significant two-way interaction (pretreatment $\times$ time $), F(2.26,22.56)=8.96, p=.001, \eta_{\mathrm{p}}{ }^{2}=.472$. Locomotor activity changed significantly as a function of time for the MDMA 10.0 group, $F(1.66,6.67)=10.14, p=.011, \eta_{\mathrm{p}}{ }^{2}=.717$, but not the MDMA 0.0 group $(p>.05)$. MDMA-produced locomotor hyperactivity $15-30$ min following MDMA was significantly greater in the MDMA 10.0 group $(p<.05)$. Total MDMA-produced locomotor activity was also significantly greater in the MDMA 10.0 group, $t(10)=3.01, p=.013, d=1.62$.

Microinjections of MDMA into the lateral C-P produced minimal locomotor hyperactivity regardless of pretreatment condition. The two-way interaction (pretreatment $x$ time) was not significant $(p>.05)$ and there was no main effect of pretreatment $(p>.05)$. There was a significant main effect of time, $F(3.00,20.97)=6.25, p=.003, \eta_{\mathrm{p}}^{2}=.471$, with significant MDMA-produced locomotor hyperactivity produced 5 and 15 min following 
MDMA. There was no significant difference in total MDMA-produced locomotor activity between the MDMA 0.0 and 10.0 groups $(p>.05)$.

Microinjections of MDMA into the NAcc produced rearing behaviour in both groups. There was a significant two-way interaction (pretreatment $\times$ time), $F(4.78,66.87)=6.70, p<$ $.001, \eta_{\mathrm{p}}{ }^{2}=.324$. Rearing changed significantly as a function of time for both the MDMA 0.0 , $F(3.50,20.97)=7.07, p=.001, \eta_{\mathrm{p}}^{2}=.574$ and MDMA 10.0, $F(3.50,27.92)=15.52, p<.001$, $\eta_{\mathrm{p}}{ }^{2}=.660$ pretreatment groups. MDMA-produced rearing was both of greater magnitude and longer duration for the MDMA 10.0 group (see Figure 3.4, $p<.05$ ). Multiple small, but significant differences in baseline rearing activity were also observed (see Figure 3.4, $p<.05$ ). Total MDMA-produced rearing was significantly greater in the MDMA 10.0 group, $t(14)=$ $4.05, p=.001, d=2.16$.

Microinjections of MDMA into the medial C-P only produced rearing in rats pretreated with MDMA 10.0. There was a significant two-way interaction (pretreatment $\times$ time), $F(3.39$, $33.92)=5.61, p=.002, \eta_{\mathrm{p}}^{2}=.360$. Locomotor activity changed significantly as a function of time for the MDMA 10.0 group, $F(1.96,7.83)=6.27, p=.024, \eta_{\mathrm{p}}{ }^{2}=.610$, but not the MDMA 0.0 group $(p>.05)$. MDMA-produced rearing 20 - 30 min following MDMA was significantly greater in the MDMA 10.0 group $(p<.05)$. Differences between the groups in total MDMAproduced rearing behaviour were only approaching significance, however $(p=.060)$.

Microinjections of MDMA into the lateral C-P produced minimal rearing regardless of pretreatment condition. The two-way interaction (pretreatment $\times$ time) was not significant $(p$ $>.05)$ and there was no main effect of pretreatment $(p>.05)$ or time $(p>.05)$. There was no significant difference in total MDMA-produced rearing between the MDMA 0.0 and 10.0 groups $(p>.05)$. 

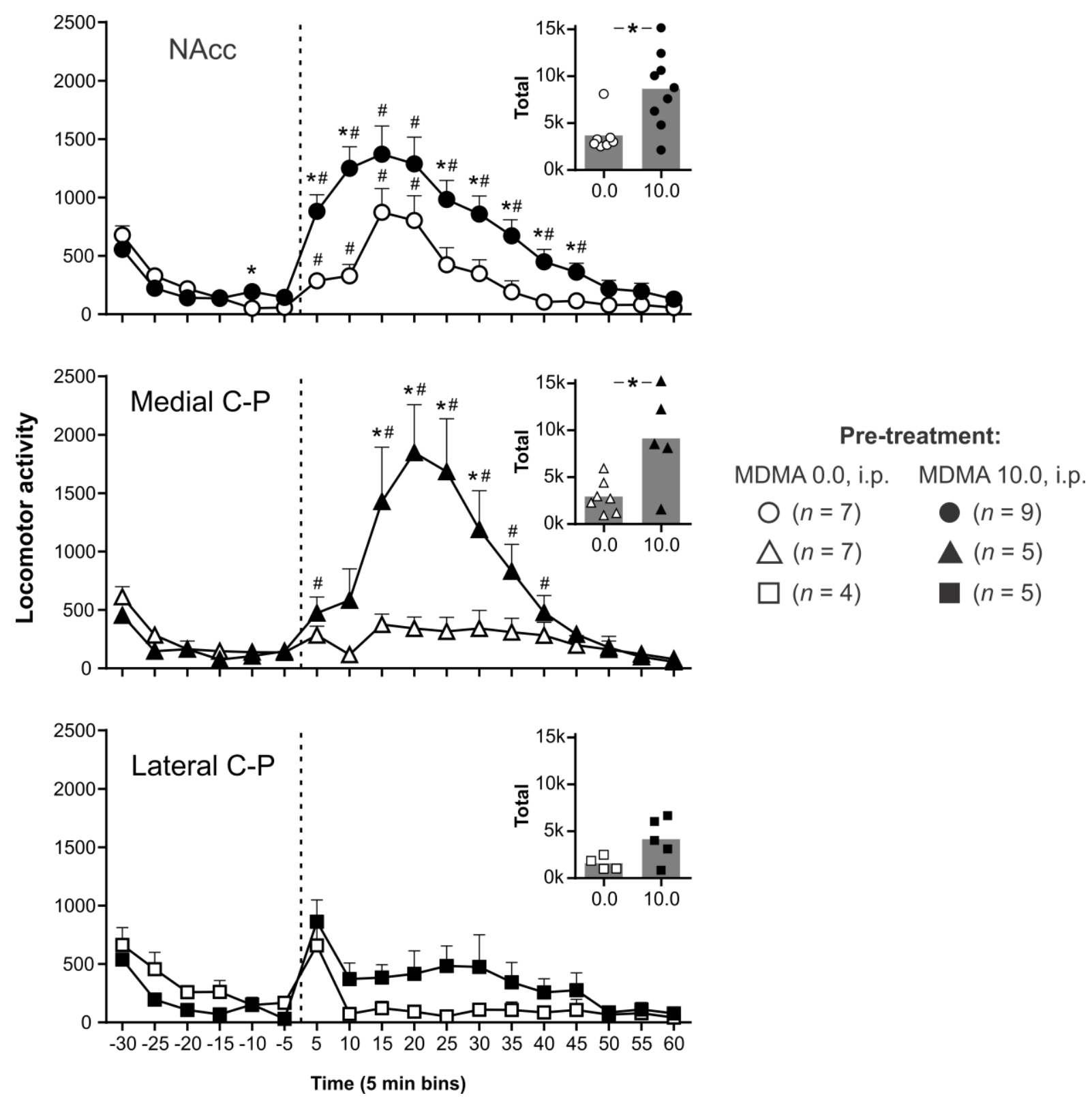

Figure 3.3. Mean (+ standard error of the mean) locomotor activity produced by local MDMA infusions $(200 \mu \mathrm{g} / 1 \mu \mathrm{L} /$ side) into the nucleus accumbens (NAcc; $\odot$, •), medial caudateputamen (C-P; $\Delta, \boldsymbol{\Delta})$, and lateral C-P ( $\square, \boldsymbol{\square}$, ) as a function of session time ( 5 min bins), following pretreatment with $0.0(\circ, \Delta, \square)$ or $10.0(\bullet, \boldsymbol{\Lambda}, \boldsymbol{\square}) \mathrm{mg} / \mathrm{kg}$ i.p. MDMA. MDMA was administered at time $=0 \mathrm{~min}$. Inserts display the mean total MDMA-produced locomotor activity from $0-60 \mathrm{~min}$, with symbols representing individual subject data. $* p<.05$ compared to MDMA 0.0. ${ }^{\#} p<.05$ compared to -5 min time point for the same group. 

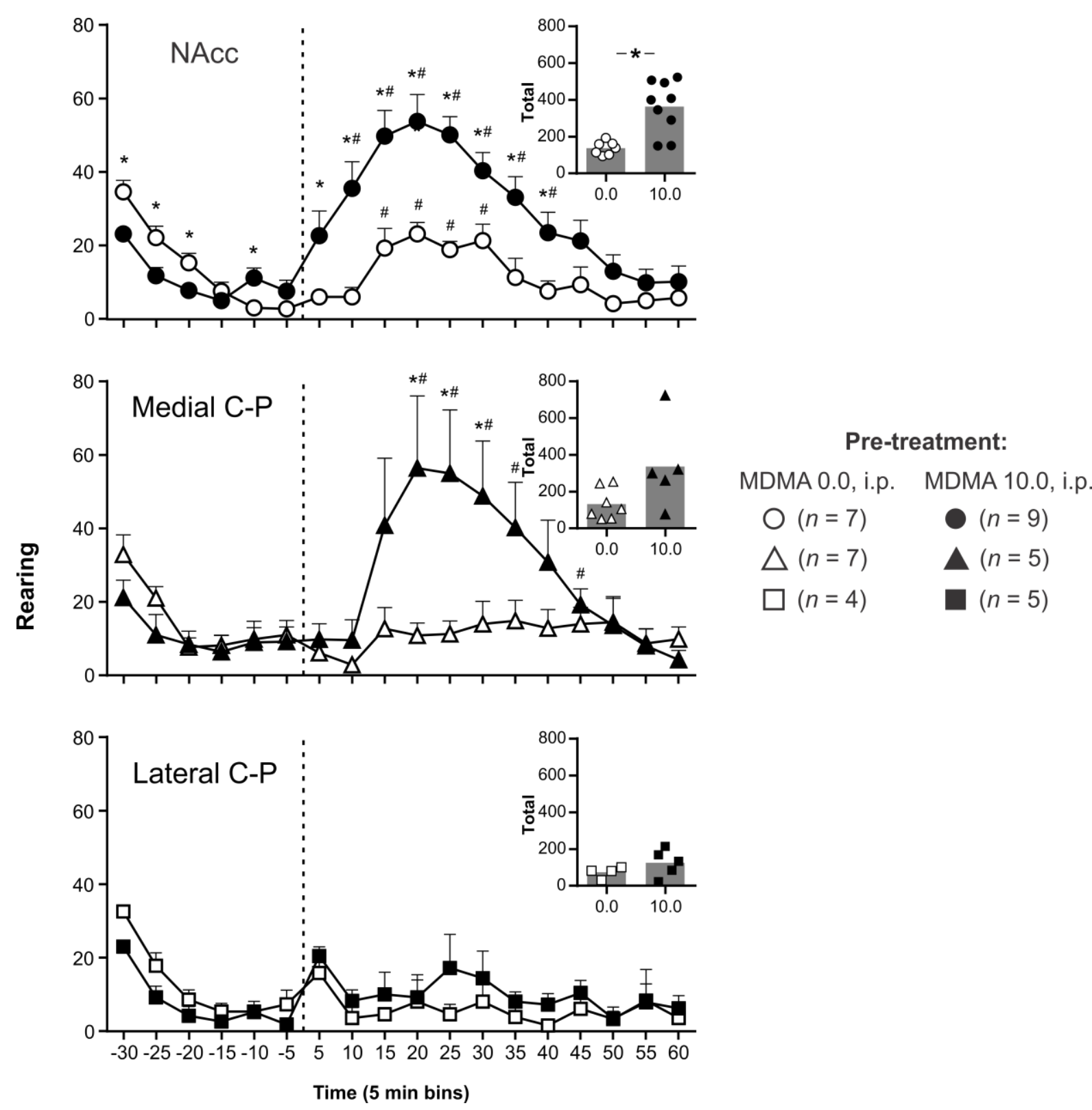

Figure 3.4. Mean (+ standard error of the mean) rearing behaviour produced by local MDMA infusions $(200 \mu \mathrm{g} / 1 \mu \mathrm{L} /$ side) into the nucleus accumbens (NAcc; $\odot$, •), medial caudateputamen (C-P; $\Delta, \boldsymbol{\Delta})$, and lateral C-P ( $\square, \boldsymbol{\square}$, ) as a function of session time ( 5 min bins), following pretreatment with $0.0(\circ, \Delta, \square)$ or $10.0(\bullet, \boldsymbol{\Lambda}, \boldsymbol{\square}) \mathrm{mg} / \mathrm{kg}$ i.p. MDMA. MDMA was administered at time $=0 \mathrm{~min}$. Inserts display the mean total MDMA-produced locomotor activity from $0-60 \mathrm{~min}$, with symbols representing individual subject data. $* p<.05$ compared to MDMA 0.0. ${ }^{\#} p<.05$ compared to -5 min time point for the same group. 


\section{Discussion}

In this study, the effect of a sensitising pretreatment regimen of repeated systemic MDMA exposure on the subsequent locomotor activating effect of MDMA administered directly into the ventral, dorsomedial, and dorsolateral striatum was determined. Both horizontal and vertical (rearing) locomotor activity measures showed similar results; therefore, these findings will be discussed together. Local administration of MDMA into the ventral (NAcc), but not the dorsal striatum (medial C-P or lateral C-P) produced significant locomotor hyperactivity in drug naïve subjects, which is consistent with what has been found with other psychostimulant drugs (Carr \& White, 1987; Delfs, Schreiber, \& Kelley, 1990; Kelley, Gauthier, \& Lang, 1989). In MDMA pretreated subjects, locomotor hyperactivity produced by local administration of MDMA into the NAcc was enhanced, and significant hyperactivity was produced by microinjections of MDMA into the medial, but not lateral, C-P. These results are consistent with those from previous studies that have demonstrated the expression of behavioural sensitisation following the local administration of amphetamine into the NAcc or dorsal striatum (Kolta, Shreve, \& Uretsky, 1989; Paulson \& Robinson, 1991). These findings suggest that similar brain regions are involved in the acute and sensitised locomotor activating effect of MDMA as other psychostimulant drugs.

The current study did not intend to extensively characterise the various brain regions involved in the expression of MDMA behavioural sensitisation. Rather, a few key brain regions were selected based on the results from Chapter 2 where it was demonstrated that the medial and ventral areas, but not the lateral areas of the striatum showed significant increases in $\Delta F$ osB levels following MDMA self-administration. As was predicted, local infusion of MDMA into these same brain regions also resulted in the expression of behavioural sensitisation. These results highlight the behavioural relevance of the region-specific increases in striatal $\Delta$ FosB observed following repeated MDMA exposure. Together, these findings suggest that MDMAproduced increases in $\triangle \mathrm{Fos} B$ within these brain regions might be involved in the development and/or expression of sensitised responses to MDMA following repeated exposure, as has been shown with other drugs of abuse (Brenhouse \& Stellar, 2006; Colby et al., 2003; Conversi et al., 2007; Grueter et al., 2013; Kaplan et al., 2011; Kelz et al., 1999; Marttila et al., 2006; McDaid et al., 2006; Zachariou et al., 2006).

A handful of previous studies have similarly demonstrated an important role of striatal mechanisms in the development and expression of MDMA behavioural sensitisation. Repeated exposure to MDMA under conditions that produced behavioural sensitisation also increased 
the firing rate of dorsal striatal neurons (Ball et al., 2006) and increased dendritic spine density in accumbal MSN (Ball et al., 2009). Both 5-HTergic and DAergic mechanisms within the striatum have also been implicated. Tissue levels of 5-HT in the striatum were decreased, while NAcc DA levels were increased following a sensitising regimen of repeated MDMA exposure (Mayerhofer et al., 2001). MDMA-produced increases in extracellular concentrations of DA in the NAcc were also increased following a sensitising regimen of repeated MDMA exposure (Kalivas et al., 1998). These findings support the results of the current study implicating an important role of these brain regions in the expression of MDMA behavioural sensitisation.

In summary, the current findings extend those of the previous chapter, highlighting the medial striatum (NAcc and DMS) as an important brain region impacted by repeated MDMA exposure and the relevance of this brain region to the expression of sensitised behavioural responses to MDMA. 


\section{CHAPTER 4: MICRODIALYSIS AND LC-MS}

\section{Introduction}

In Chapter 2, the impact of repeated MDMA exposure on the accumulation of the transcription factor, $\Delta \mathrm{FosB}$, in numerous brain regions was determined. A key finding was the region-specific differences within the striatum; repeated MDMA exposure produced significant increases in $\triangle \mathrm{FosB}$ in the medial, but not the lateral regions of the striatum. The behavioural relevance of these findings was examined in Chapter 3; behavioural sensitisation following repeated systemic MDMA exposure was expressed following local infusions of MDMA into the medial, but not lateral striatum. In the current chapter, an investigation of the more specific neurochemical changes that occur within the medial striatum following a sensitising regimen of repeated MDMA exposure was carried out.

MDMA is a potent releaser and reuptake inhibitor of both 5-HT and DA (Battaglia, Brooks, et al., 1988; Baumann, Clark, Franken, et al., 2008; Baumann, Clark, \& Rothman, 2008; Berger et al., 1992; Cole \& Sumnall, 2003; Fitzgerald \& Reid, 1990; Hekmatpanah \& Peroutka, 1990; Iravani et al., 2000; Kankaanpää et al., 1998; Kurling et al., 2008; Nair \& Gudelsky, 2004; Nash \& Brodkin, 1991; O’Shea et al., 2005; Rudnick \& Wall, 1992; Shankaran \& Gudelsky, 1999). Considerable evidence suggests that 5-HTergic neurotransmission becomes compromised following substantial exposure to MDMA. For example, decreased 5-HT tissue levels, decreased 5-HT re-uptake sites, and decreased synaptic overflow of 5-HT have all been observed following repeated MDMA exposure (Battaglia, Yeh, et al., 1988; Battaglia et al., 1987; Commins et al., 1987; Do \& Schenk, 2013; Mayerhofer et al., 2001; O’Hearn et al., 1988; Reveron et al., 2010; Schenk et al., 2007; Shankaran \& Gudelsky, 1999). These 5-HT deficits appear to be dependent on exposure levels, however, as repeated exposure to lesser amounts of MDMA failed to produce these deficits (Banks et al., 2008; Do \& Schenk, 2013; Fantegrossi et al., 2004). It is not currently known whether such 5HTergic deficits develop as a function of repeated exposure to MDMA under conditions that produce behavioural sensitisation.

In contrast, there is some evidence to suggest that repeated, intermittent MDMA exposure can sensitise DAergic neurotransmission, and that this might contribute to the development and expression of MDMA sensitisation. For example, tissue levels of DA were increased (Mayerhofer et al., 2001) and the capacity for MDMA to produce increases in NAcc DA was enhanced following a sensitising regimen of repeated MDMA exposure (Kalivas et 
al., 1998). An extensive characterisation of both the DAergic and 5-HTergic correlates of sensitised MDMA-produced behaviour has not been previously carried out, however, and was, therefore, the primary aim of the current study.

This can be achieved using microdialysis and LC-MS. Microdialysis can be used to sample the constituents of extracellular fluid in vivo. Samples can be collected relatively frequently and importantly, can also be collected from awake, freely moving animals, allowing for the concurrent collection of behavioural data. With the appropriate sample preparation procedures, LC-MS can be used to detect and quantify numerous compounds of interest contained within microdialysate samples with good sensitivity, good selectivity, and all within in a single analysis, as has been previously demonstrated (Song, Mabrouk, Hershey, \& Kennedy, 2012; Wong et al., 2016).

Initial attempts to develop in-house microdialysis and LC-MS procedures for the analysis of DA and 5-HT were previously performed by another lab member that had adapted a sample preparation method using benzoyl chloride (BzCl) derivatisation (Song et al., 2012; Wong et al., 2016). BzCl derivatises most small molecules present in microdialysis samples, reacting with both primary and secondary amines, phenols, and ribose-hydroxyl groups (Figure 4.1; Song et al., 2012; Wong et al., 2016). Derivatisation of dialysates with $\mathrm{BzCl}$ has been shown to increase detection sensitivity, decrease degradation, and render compounds more hydrophobic, which can improve reversed-phase chromatography (Song et al., 2012; Wong et al., 2016). This allows for the quantification of many small neurotransmitters, neuromodulators, and metabolites in a single LC-MS analysis, which is ideal for the current purposes (Song et al., 2012; Wong et al., 2016). Although initial testing using this procedure showed some promise, it was clear that many issues with the procedure needed to be addressed and optimised. The process of further developing and refining this procedure as well as the application of the procedure for the investigation of neurochemical changes following a sensitising regimen of repeated MDMA exposure is described in this chapter. 


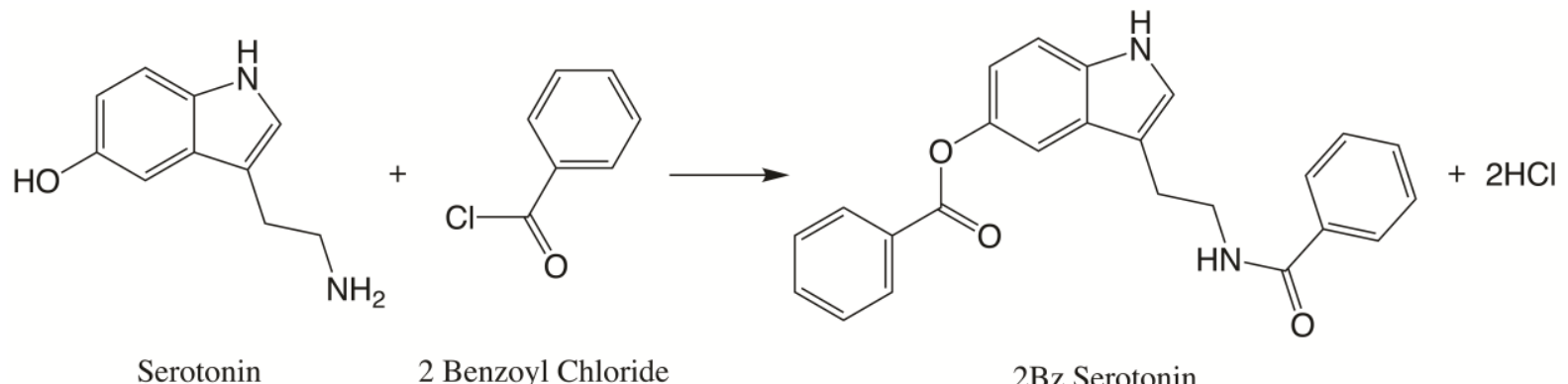

2Bz Serotonin

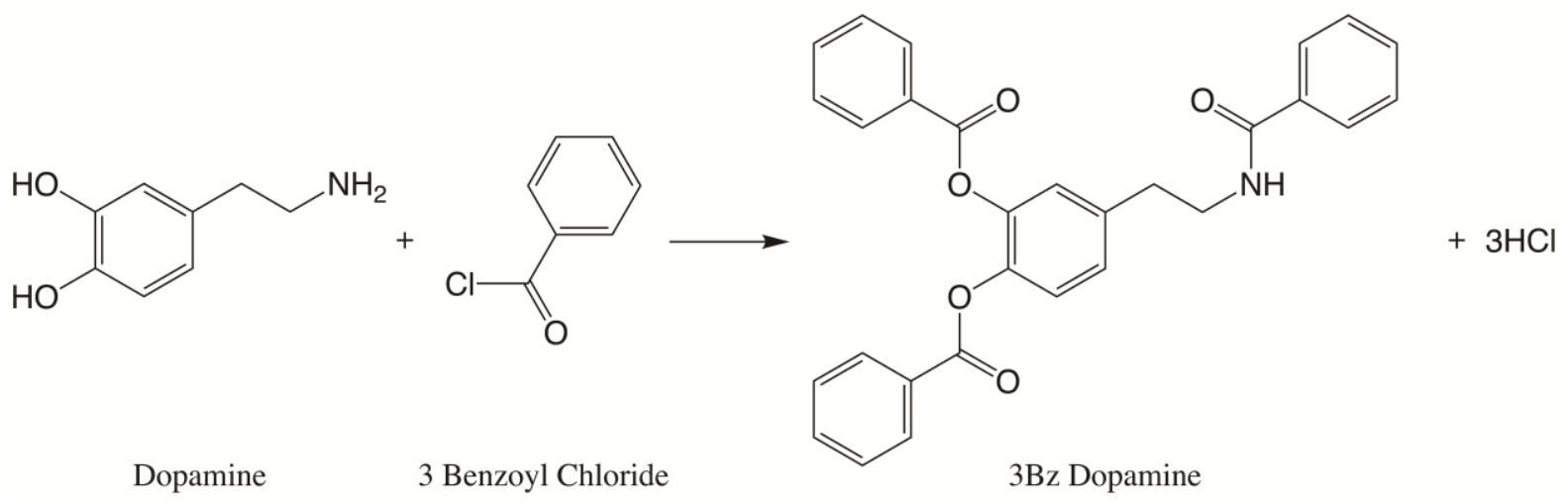

Figure 4.1. Benzoyl chloride derivatisation of serotonin and dopamine.

\section{Methods 1}

\section{Subjects}

Male Sprague-Dawley rats $(n=25)$ were bred and housed as described in Chapter 2. All experimental protocols were approved by the Animal Ethics Committee of Victoria University of Wellington.

\section{Drugs and reagents}

\pm MDMA $\mathrm{HCl}$ (BDG synthesis, New Zealand) was dissolved in a sterile $0.9 \% \mathrm{NaCl}$ solution for i.p. injections. aCSF $\left(137 \mathrm{mM} \mathrm{NaCl}, 2.7 \mathrm{mM} \mathrm{KCl}, 1.2 \mathrm{mM} \mathrm{CaCl}_{2}, 0.5 \mathrm{mM} \mathrm{MgCl}\right.$, $8.1 \mathrm{mM} \mathrm{Na}_{2} \mathrm{HPO}_{4}, 1.5 \mathrm{mM} \mathrm{KH} \mathrm{PO}_{4}, \mathrm{pH}$ 7.4) was used as dialysis perfusate and for standard dilutions. Standard stock solutions were made from DA $\mathrm{HCl}, 5-\mathrm{HT} \mathrm{HCl}$, 3,4dihydroxyphenylacetic acid (DOPAC), 5-HIAA, D2 DA HCl, and D2 5-HT HCl dissolved in $\mathrm{H}_{2} \mathrm{O}$ at $1 \mathrm{mM}$ and kept at $-80{ }^{\circ} \mathrm{C}$ in $1 \mathrm{~mL}$ aliquots. All chemicals used for the preparation of standards were purchased from Sigma Aldrich except for D2 5-HT, which was synthesised inhouse. All water used for dialysis and LC-MS was distilled and deionized using a MilliQ system. Acetonitrile was LC-MS grade and purchased from Fisher Scientific. Ammonium formate, formic acid, sodium tetraborate, and $\mathrm{BzCl}$ were purchased from Sigma Aldrich. 


\section{Surgical procedures}

Pre- and post-operative surgical procedures were carried out as described in Chapter 2. Intracerebral guide cannula containing dummy cannula (MAB 4.15.IC, Microbiotech, Sweden) were stereotaxically implanted in the medial striatum at the following coordinates; $+1.4 \mathrm{~mm}$ AP, -2.0 mm ML, -3.6 mm DV. All coordinates were relative to bregma/skull with incisor bar $-3.9 \mathrm{~mm}$ from the interaural line (Paxinos \& Watson, 2005). Guide cannulae were secured in place with screws and dental acrylic.

\section{Microdialysis}

Testing was conducted within the home cage or the same locomotor activity chambers described in Chapter 3. Microdialysis probes (MAB 4.15.2.Cu, Microbiotech, Sweden) with a membrane surface area of $0.24 \mathrm{~mm} \times 2 \mathrm{~mm}$ (diameter $\times$ length) were inserted into the guide cannula, and aCSF was perfused overnight at a flow rate of $1 \mu \mathrm{L} / \mathrm{min}$ using a microinfusion pump (PHD 2000 infusion, Harvard Apparatus). These probes had a relative recovery of approximately $15 \%$, as determined in vitro at $1 \mu \mathrm{L} / \mathrm{min}$. The following morning (approximately 12 hours later), baseline dialysis samples were collected at 20 min intervals for 1 hour within the home cage, 1 hour in the locomotor activity chambers, then for 2 hours following cumulative doses of MDMA $(5.0,10.0 \mathrm{mg} / \mathrm{kg}$, i.p). A derivatisation procedure adapted from (Song et al., 2012; Wong et al., 2016) was used. To each $20 \mu \mathrm{L}$ dialysate sample, $1.25 \mu \mathrm{L}$ of internal standard (50 nM D2 DA/D2 5-HT), $10 \mu \mathrm{L}$ of borate buffer (sodium tetraborate, 100 $\mathrm{nM}$ ) and $10 \mu \mathrm{L}$ of $\mathrm{BzCl}$ (diluted to $5 \%$ [v/v] in acetonitrile) was added, with mixing between each step. The sample mixture was then vortexed for $2 \mathrm{~min}$ and stored at $-80{ }^{\circ} \mathrm{C}$ until analysed.

At the end of testing, animals were euthanised by $\mathrm{CO}_{2}$ asphyxiation. Brains were rapidly removed and placed in $4 \%$ PFA fixative overnight before being frozen at $-80^{\circ} \mathrm{C}$ until sectioning. Coronal sections $(100 \mu \mathrm{m})$ were cut along the striatum using a sliding microtome (HM 450 with KS34 freezing unit, Thermo Scientific). Sections were then mounted on gelatincoated slides, stained with $1 \%$ neutral red, and cover-slipped with DPX mounting medium. Light microscopy was used to verify the location of the probe membranes. Data from rats with placements outside of the target area were excluded from any analysis.

\section{LC-MS}

Analysis of microdialysate samples was carried out using an Agilent Technologies (Palo Alto) 6530 quadrupole time-of-flight (Q-TOF) LC-MS equipped with a Jet-Stream electrospray ionisation (ESI) source. Derivatised sample $(40 \mu \mathrm{L})$ was injected by an autosampler kept at $4{ }^{\circ} \mathrm{C}$ onto a Poroshell $120 \mathrm{SB}-\mathrm{Aq} 2.7 \mu \mathrm{m}$ column $(2.1 \times 100 \mathrm{~mm}$; Agilent 
Technologies) held at $36{ }^{\circ} \mathrm{C}$. Eluent A was $99.9 \%$ water/0.1\% ammonium formate. Eluent B was $99.9 \%$ acetonitrile $/ 0.1 \%$ formic acid. Liquid chromatography was performed with the following gradient: $15 \%$ eluent B for $1 \mathrm{~min}, 40 \%$ at $3 \mathrm{~min}, 50 \%$ at $6 \mathrm{~min}, 97.5 \%$ at $7 \mathrm{~min}$, held at $97.5 \%$ until $10 \mathrm{~min}$, and reduced to $15 \%$ at $11 \mathrm{~min}$. The flow rate was $0.45 \mathrm{~mL} / \mathrm{min}$ until 7 min, increased to $0.5 \mathrm{~mL} / \mathrm{min}$ from $8-10 \mathrm{~min}$, and reduced back to $0.45 \mathrm{~mL} / \mathrm{min}$ at $11 \mathrm{~min}$. A post-run time was set to $3.5 \mathrm{~min}$. Q-TOF ESI-MS parameters were as follows: positive ion mode; gas temperature, $275^{\circ} \mathrm{C}$; gas flow, $8 \mathrm{~L} / \mathrm{m}$; nebulizer, 30 psi; capillary voltage, $2750 \mathrm{~V}$; nozzle voltage, $0 \mathrm{~V}$; fragmentor voltage, $130 \mathrm{~V}$; acquisition rate, 2 spectra/s; mass range, $\mathrm{m} / \mathrm{z}$ $50-1100$.

\section{Data analysis}

An external standard mixture was serially diluted (v/v) from the frozen stock solutions in aCSF at $0,0.5,1,5$, and $10 \mathrm{nM}$ for DA/5-HT and 0, 5, 10, 50, and $100 \mathrm{nM}$ for DOPAC/5HIAA. External standards were prepared daily alongside the collection of each batch of dialysate samples and acted as pseudo quality controls. D2 DA and D2 5-HT were used as internal standards, and a mixture was diluted daily to $50 \mathrm{nM}$ in aCSF and spiked in each standard/dialysate sample to a final concentration of $2.94 \mathrm{nM}$. Automated peak integration of the targeted analytes was performed by the MassHunter Workstation Quantitative software with visual inspection to ensure proper integration. Analyte concentration was determined by calibration curves made from external standards analysed in triplicate with the peak area response ratios (external standard / internal standard) plotted against the internal standard concentration ratios (expected external standard concentration / expected internal standard concentration) using a weighted $\left(1 / \mathrm{SD}^{2}\right)$ linear model (Almeida, Castel-Branco, \& Falcão, 2002; Moosavi \& Ghassabian, 2018).

\section{Results 1}

Unfortunately, complete data were only obtained from a small proportion of the subjects that were tested. Several issues with the microdialysis equipment prevented the collection of dialysate samples from 9 subjects. These issues included probe/tubing disconnections, blockages, and tangles, which were remedied partway through the study with the development of new sampling equipment and procedures. Unexpected technical difficulties with the LC-MS and other analysis issues also prevented that analysis of samples from another 6 subjects. Thus, complete data were only obtained for 11 of the 25 subjects. Calculated concentrations of DA, 5-HT, DOPAC, and 5-HIAA for a representative subject are shown in Figure 4.2. DA and 5-HT increased dose-dependently following MDMA administration as 
expected. In contrast, DOPAC decreased dose-dependently, while concentrations of 5-HIAA did not change, as previously demonstrated (Gough, Imam, Blough, Slikker, \& Ali, 2006; Nash, 1990).
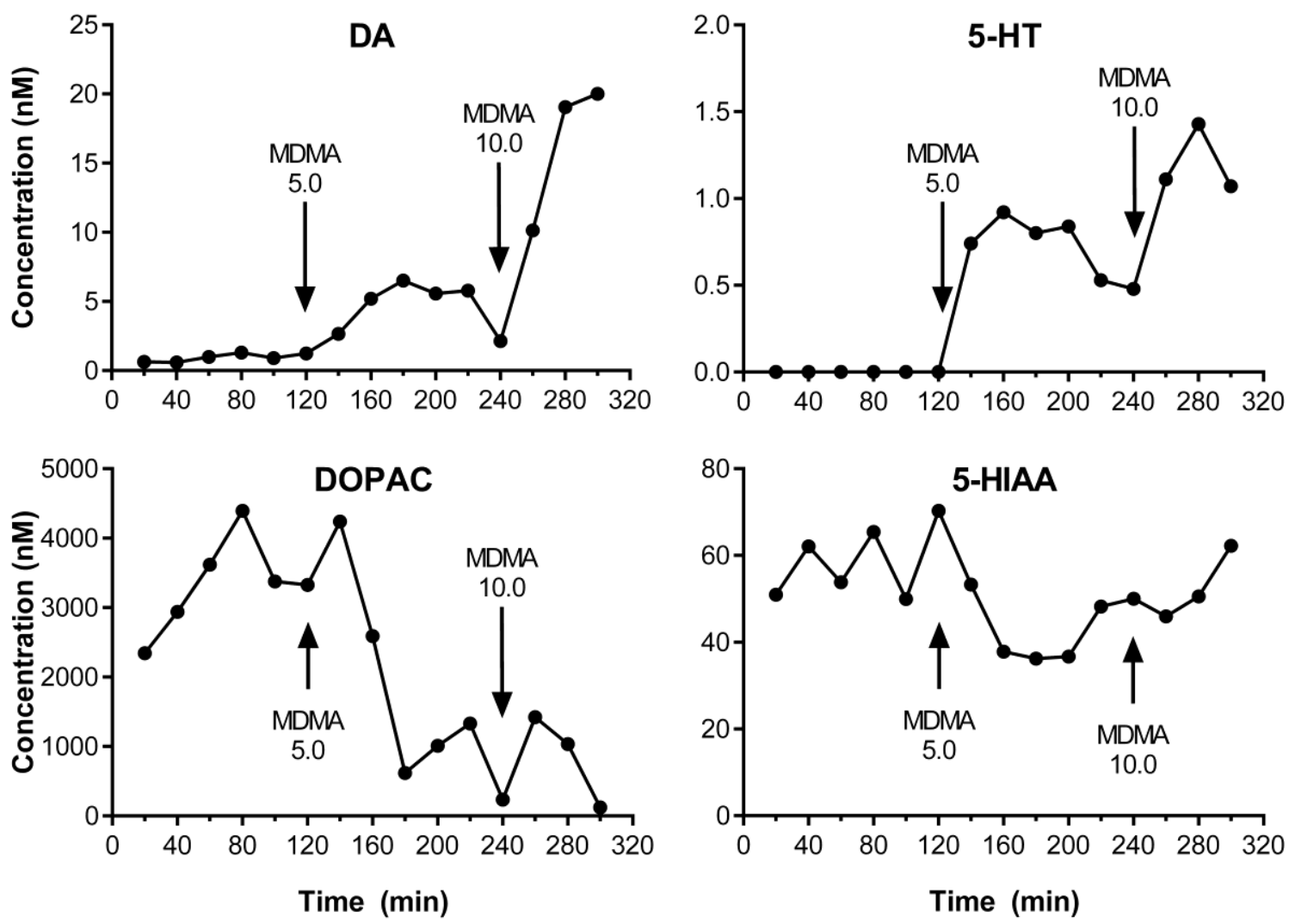

Figure 4.2. Calculated concentrations of DA, 5-HT, DOPAC, and 5-HIAA as a function of time/MDMA administration for a representative subject. Microdialysis samples were collected at $20 \mathrm{~min}$ intervals. MDMA 5.0 and 10.0 (mg/kg, i.p.) was administered at $120 \mathrm{~min}$ and 240 min, respectively. 
Of the data that were collected and analysed correctly, it was clear that several other issues also needed to be addressed. Calibration curves for the standards were not acceptable, with low $\mathrm{R}^{2}$ and poor repeatability. Dialysate concentrations of 5-HT and DA during baseline conditions were also incredibly low, and the peaks were well below the limits of detection (Figure 4.3). As can also be seen in Figure 4.3, a co-eluting peak of similar m/z to DA also prevented the accurate integration of DA.

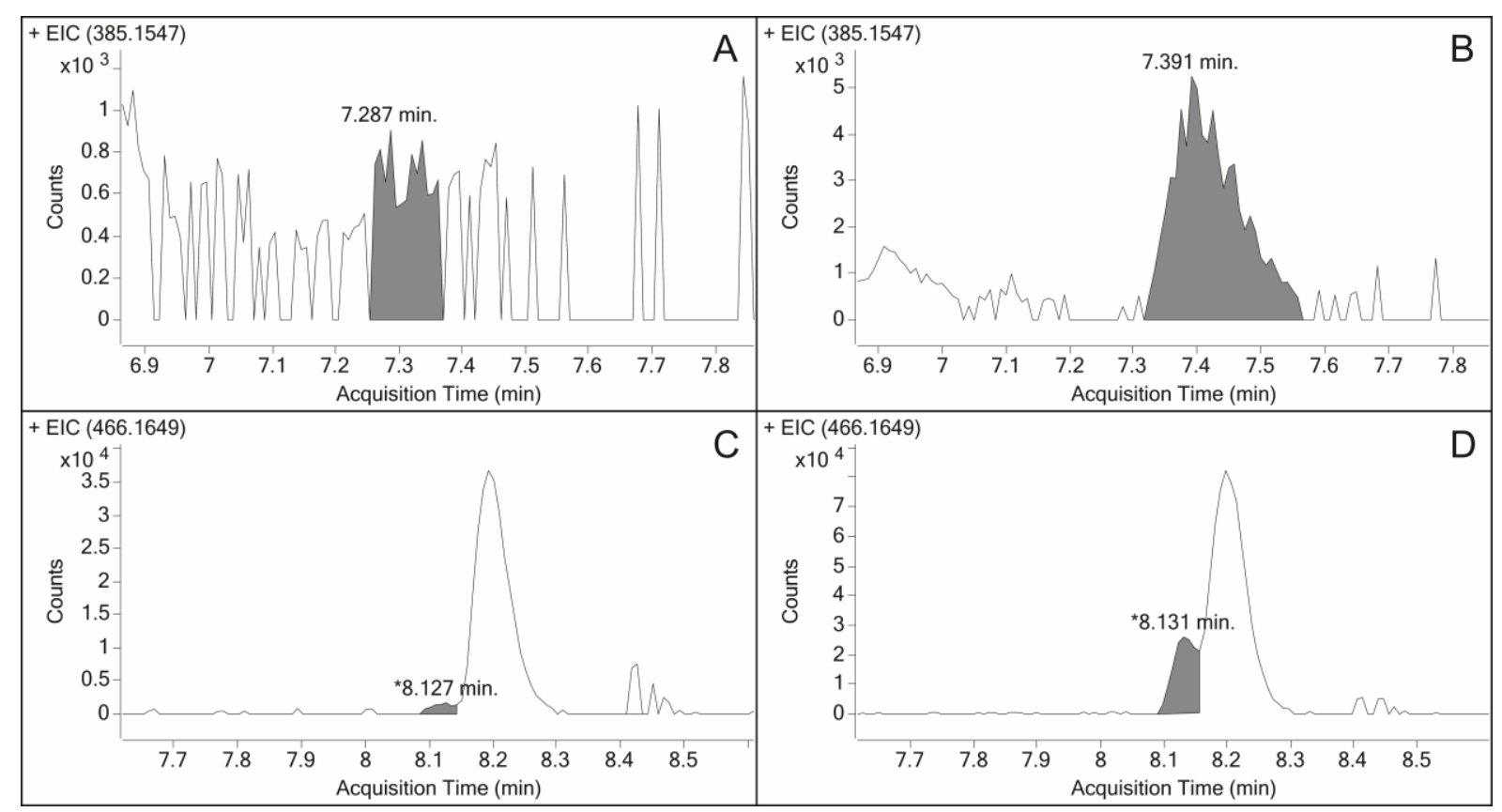

Figure 4.3. Extracted ion chromatograms of derivatised serotonin (panels A and B) and dopamine (panels $\mathrm{C}$ and $\mathrm{D}$ ) in rat brain microdialysate samples during baseline conditions (panels A and C) and following $10 \mathrm{mg} / \mathrm{kg}$ i.p. MDMA (panels B and D). 


\section{Method development 1}

\section{Chromatography gradient}

The first issue that needed to be addressed was the co-eluting peak of similar $\mathrm{m} / \mathrm{z}$ to DA. This peak was not present in standards or aCSF and was subsequently identified to be a possible contaminant from either the tubing, probes, or filters used during the collection and preparation of dialysate samples. Rather than trying to identify the exact source and eliminate the contaminant, the chromatography gradient was changed to alter the retention time of DA and the containment, while maintaining the adequate retention of the other targets. The new gradient that achieved this was as follows: $30 \%$ eluent B for $0.5 \mathrm{~min}, 97.5 \%$ at $9 \mathrm{~min}$, held at $97.5 \%$ until $10 \mathrm{~min}, 50 \%$ at $10.5 \mathrm{~min}, 97.5 \%$ at $10.7 \mathrm{~min}$, held at $97.5 \mathrm{until} 11.5 \mathrm{~min}, 30 \%$ at $11.7 \mathrm{~min}$. The flow rate was $0.45 \mathrm{~mL} / \mathrm{min}$ until $10.7 \mathrm{~min}$, increased to $0.5 \mathrm{~mL} / \mathrm{min}$ from 10.7 $11.7 \mathrm{~min}$, and reduced back to $0.45 \mathrm{~mL} / \mathrm{min}$ at $11.7 \mathrm{~min}$.

\section{$\mathrm{BzCl}$ concentration}

The second issue that needed to be addressed was the low analytical sensitivity for DA and 5-HT. It was discovered that a lower concentration of $\mathrm{BzCl}$ used for sample derivatisation ( $2 \%$ instead of $5 \%$ ) greatly improved the sensitivity for all target analytes, as indicated by a significant increase in the slope of the calibration curve (Figure 4.4). A validation procedure was subsequently carried out to quantify the detection limits and measurement repeatability/precision of the targeted compounds using the current procedures. Calibration standards of each target analyte were prepared and analysed in triplicate once daily over three subsequent days. 8-point calibration curves were made for each target analyte on each day, and the slope, $\mathrm{R}^{2}$, limit of detection (LOD), intra-assay relative standard deviation (RSD), and intraday RSD were calculated. 

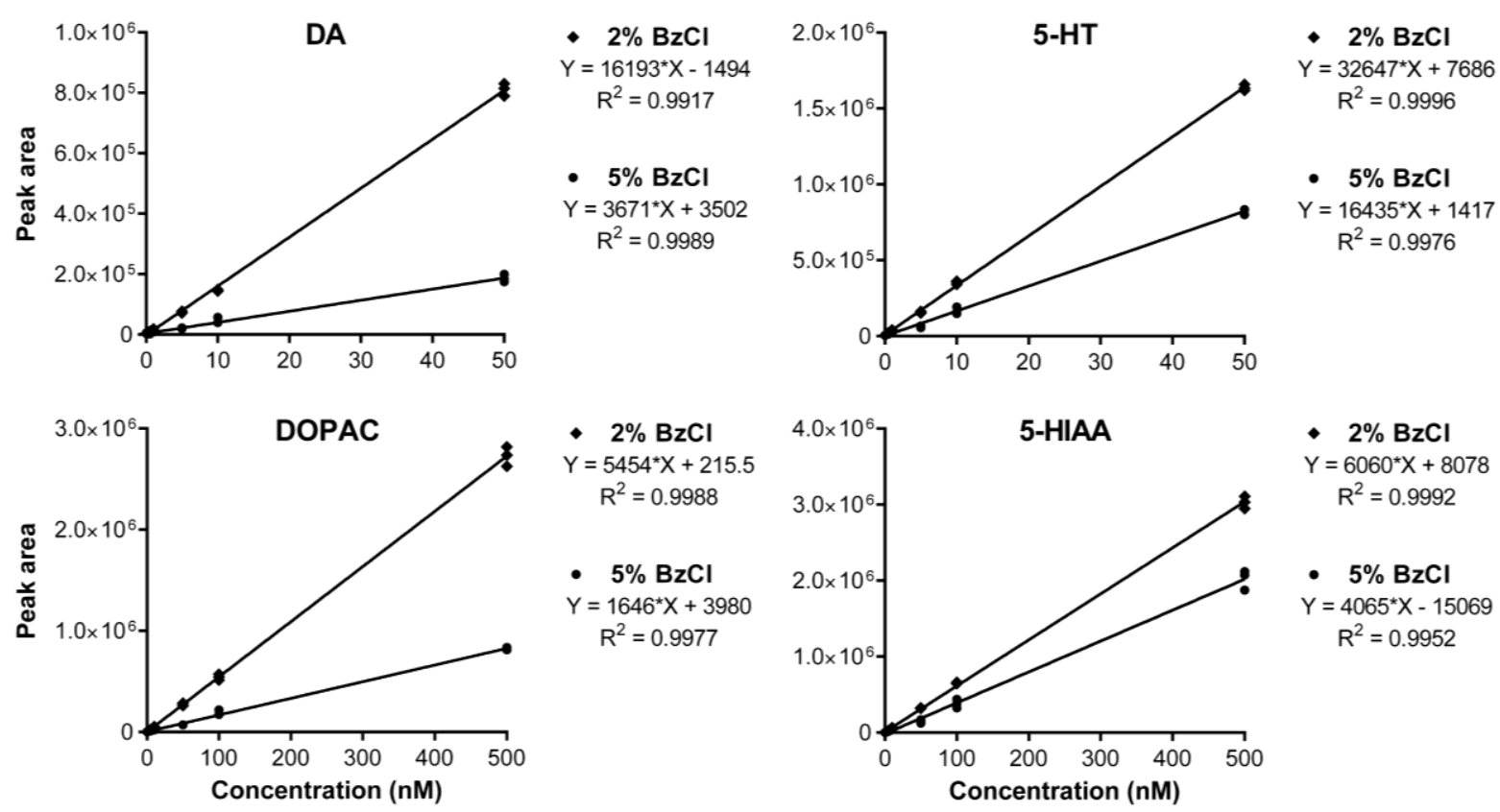

Figure 4.4. 7-point calibration curves for 5-HT, DA, 5-HIAA, and DOPAC made from external standards analysed in triplicate at 0, 0.1, 0.5, 1, 5, 10, and $50 \mathrm{nM}$ for 5-HT/DA and 0, 1, 5, 10, 50, 100, and $500 \mathrm{nM}$ for 5-HIAA/DOPAC. Standards were derivatised with either $2 \%(\diamond)$ or $5 \% \mathrm{BzCl}(\bullet)$. The slope, intercept, and $\mathrm{R}^{2}$ of each linear calibration curve are displayed in the legends.

During this process, it was realised that the internal standards were causing the calibration curves to be non-linear (Moosavi \& Ghassabian, 2018; Tan \& Awaiye, 2013). Internal standards are compounds that are added to all samples/standards. The data are then calculated as a ratio of the internal standard in order to compensate for analyte losses during any stage of sample preparation, storage, or analysis. A good internal standard will, therefore, need to have similar physicochemical properties to the target analyte and show similar behaviour throughout the entire procedure. Isotopically labelled compounds, such as those made with ${ }^{13} \mathrm{C}$ or deuterium, are ideal for this purpose. In the current study, D2 DA and D2 5HT were used, but these are only two mass units greater than the target compounds. Thus, the +2 isotope of the DA and 5-HT targets was making a sizable contribution to the peak area of the internal standards, especially when the targets were at a high concentration (Figure 4.5). This caused the calibration curves to be non-linear and the calculation of dialysate concentrations to be inaccurate (Figure 4.6A). 


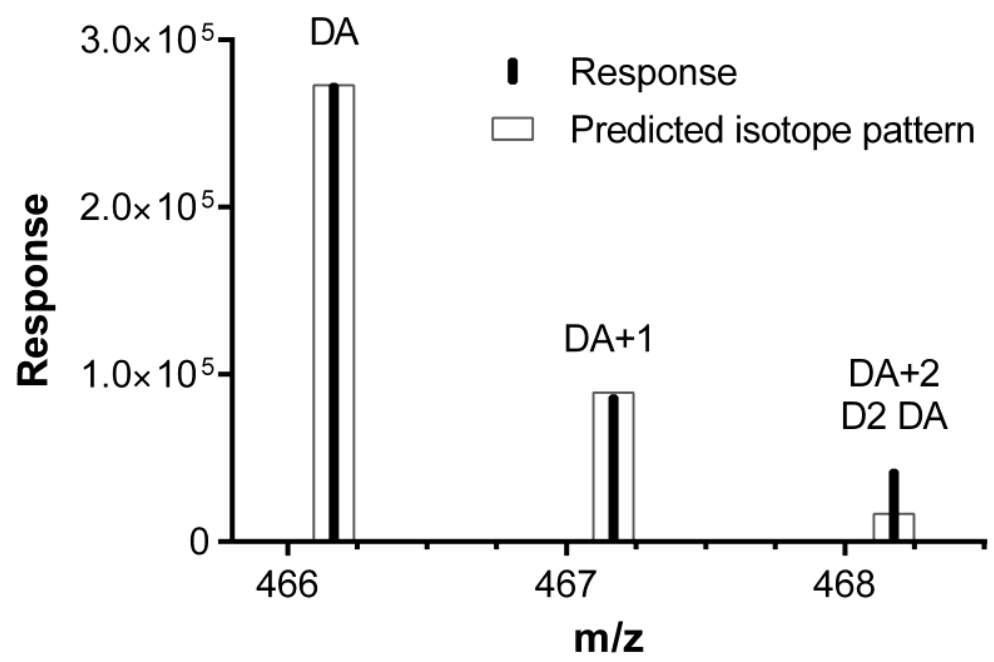

Figure 4.5. Mass spectrum showing the actual (black lines) and predicted (grey outline) isotope pattern of derivatised dopamine (DA; $466.16 \mathrm{~m} / \mathrm{z} ; 100 \mathrm{nM})$. Actual $468.17 \mathrm{~m} / \mathrm{z}$ response is considerably higher than predicted due to overlap between the DA+2 isotope and the and D2 DA (5.56 nM) internal standard.
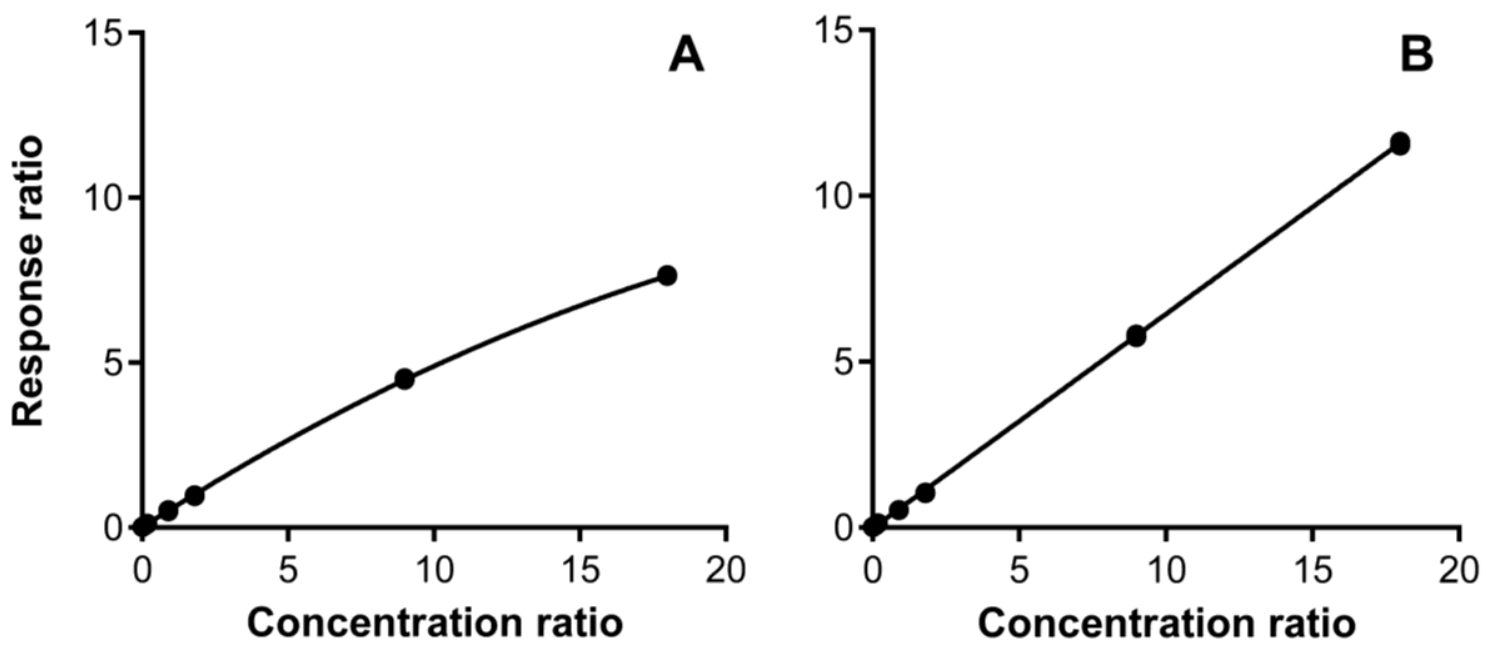

Figure 4.6. 8-point calibration curves for 5-HT made from external standards analysed in triplicate at $0,0.1,0.5,1,5,10,50$, and $100 \mathrm{nM}$ and D2 5-HT internal standard at $5.56 \mathrm{nM}$. A) Uncorrected data. B) Corrected data using $\mathrm{R}_{\mathrm{x}} / \mathrm{R}_{\mathrm{y}}$ isotopic dilution. 
To correct for this, the isotopic dilution feature in the Masshunter software was used, which requires the calculation of $R_{x}$ and $R_{y}$ where $R_{x}=$ the ratio of target to isotope in pure analyte sample and $\mathrm{R}_{\mathrm{y}}=$ the ratio of target to isotope in pure internal standard sample. The theoretical peak area contribution of the +2 isotopes of DA and 5-HT was calculated to be $6.06 \%$ and 5.24\%, respectively (Loos, Gerber, Corona, Hollender, \& Singer, 2015). Thus, for DA, $R_{x}$ was $100 / 6.06=16.5016$ and $R_{y}$ was $0 / 1=0$, while for $5-H T R_{x}$ was $100 / 5.24=24.0963$ and $\mathrm{R}_{\mathrm{y}}$ was $0 / 1=0$. This procedure largely solved the issue and resulted in linear calibration curves (Figure 4.6B). Higher concentrations of internal standards $(5.56 \mathrm{nM})$ were also used to decrease the relative contribution of the DA and 5-HT +2 isotopes (Moosavi \& Ghassabian, 2018; Tan \& Awaiye, 2013). Unfortunately, the software did not allow for this isotopic dilution procedure when an internal standard was being used for another compound (i.e. DOPAC and 5-HIAA). Thus, the DOPAC and 5-HIAA data were calculated without internal standards.

The corrected data from the aforementioned validation procedure is shown in Table 4.1. The $\mathrm{R}^{2}$ and intra-assay/intra-day RSD for DA and 5-HT was sufficient and well within accepted limits (Araujo, 2009; Moosavi \& Ghassabian, 2018). The results for 5-HIAA and DOPAC were more variable, likely due to the lack of appropriate internal standards. LODs were calculated from the peak area of 9 replicates of $0 \mathrm{nM}$ blank standards and $1 \mathrm{nM}$ (5-HT/DA) or $10 \mathrm{nM}$ (5HIAA/DOPAC) low concentration standards. The following equation was used: $\mathrm{LoD}=\mathrm{LoB}+$ 1.645 $\left(\mathrm{SD}_{\text {low concentration sample }}\right)$, where $\mathrm{LoB}=\mathrm{Mean}_{\text {blank sample }}+1.645\left(\mathrm{SD}_{\text {blank sample }}\right)$ (Armbruster \& Pry, 2008). The calculated LOD for benzoylated 5-HT and DA were equivalent to those calculated in (Wong et al., 2016) who used the same LOD calculation method. The LOD for 5-HIAA and DOPAC were considerably higher than in (Wong et al., 2016), but well below the detected levels in dialysate samples.

Table 4.1.

Validation results

\begin{tabular}{lccrrrrrr}
\hline \multirow{2}{*}{ Standard } & \multirow{2}{*}{$\mathrm{R}^{2}$} & \multicolumn{2}{c}{ LOD } & \multicolumn{2}{c}{ Intra-assay RSD $(n=3)$} & \multicolumn{3}{c}{ Inter-day RSD $(n=3)$} \\
& & $(\mathrm{nM})$ & $1 \mathrm{nM}$ & $10 \mathrm{nM}$ & $100 \mathrm{nM}$ & $1 \mathrm{nM}$ & $10 \mathrm{nM}$ & $100 \mathrm{nM}$ \\
\hline DA & 0.9980 & 0.49 & $2.3 \%$ & $2.1 \%$ & $1.1 \%$ & $5.6 \%$ & $3.9 \%$ & $1.3 \%$ \\
5-HT & 0.9976 & 0.36 & $3.6 \%$ & $1.5 \%$ & $1.2 \%$ & $4.7 \%$ & $3.4 \%$ & $2.2 \%$ \\
DOPAC*\# & 0.9911 & 24.4 & $6.5 \%$ & $5.7 \%$ & $3.7 \%$ & $15.5 \%$ & $8.9 \%$ & $16.2 \%$ \\
5-HIAA*\# & 0.9950 & 25.7 & $2.4 \%$ & $2.7 \%$ & $2.5 \%$ & $12.4 \%$ & $12.9 \%$ & $3.8 \%$ \\
\hline
\end{tabular}

*No internal standard used, ${ }^{\#}$ Concentration for RSD $\times 10$. 


\section{Probe implantation}

A third issue that needed to be addressed was the high rate of subject/data attrition. A large proportion of the data loss from the initial experiment was due to disconnections, tangles, and blockages in the microdialysis sampling equipment. Although this issue was mostly solved by a redesign of the sampling equipment and procedure, the risk could be further reduced by minimising the time from probe implantation to testing. Currently, probes were implanted 12 hours prior to testing, as is commonly done (Baumann, Clark, \& Rothman, 2008; Kalivas et al., 1998; Morgan et al., 1997). However, many studies have also used much shorter times such as 3 hours (Panos \& Baker, 2010), 2 hours (Shankaran \& Gudelsky, 1999), or even 1 hour (Benamar, Geller, \& Adler, 2008). Probe implantation causes localised tissue damage, and thus, some time between the implantation of the probe is required for extracellular concentrations of neurochemicals to stabilise. If the probe is left implanted for too long, however (2+ days), gliosis or probe blockages can occur, preventing adequate sampling.

To assess this under the current conditions, rats $(n=2)$ were implanted with microdialysis probes, and dialysate samples were immediately collected every $40 \mathrm{~min}$ for 5 hours. MDMA (10 mg/kg i.p.) was administered after 3 hours to examine dose-dependent changes in dialysate concentrations. Figure 4.7 shows that dialysate concentrations were very high during the first hour but quickly stabilised within 2 hours. In accordance with this data and with previous studies, the time between probe implantation and testing was reduced to 3 hours.
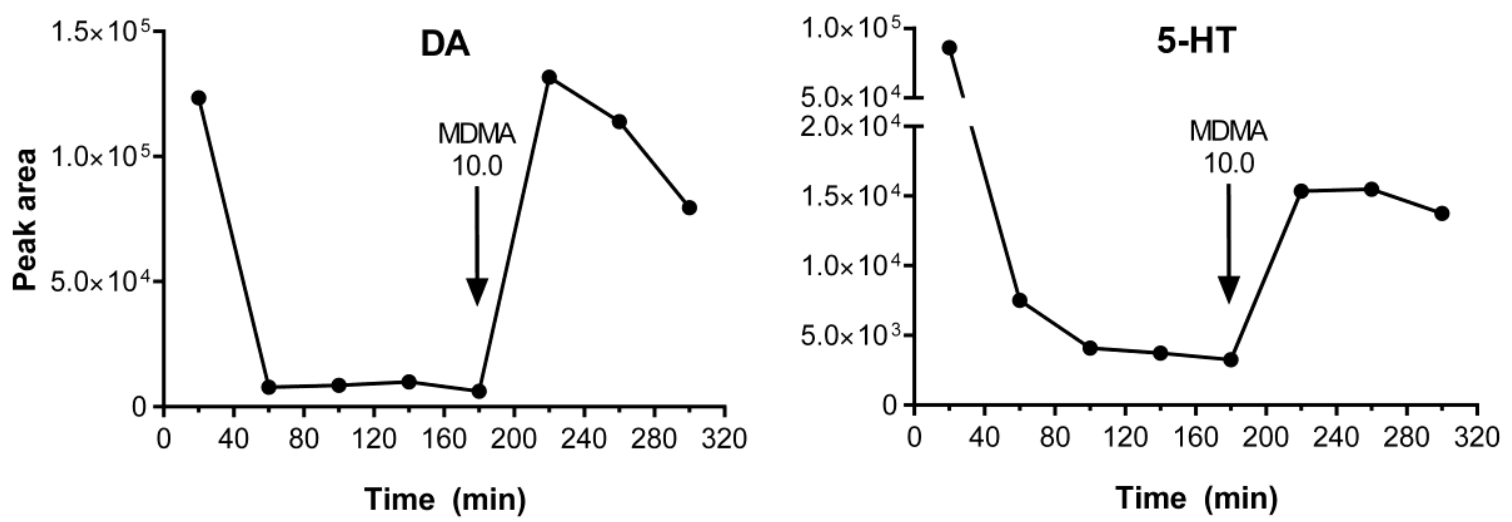

Figure 4.7. Peak area of dopamine (DA; left) and serotonin (5-HT; right) as a function of time/MDMA administration. Microdialysis samples were collected every $40 \mathrm{~min}$ following the implantation of the probe at $0 \mathrm{~min}$ and the administration of MDMA $(10.0 \mathrm{mg} / \mathrm{kg}$, i.p. $)$ at 180 $\min$. 


\section{Method 2}

Another large sample of rats $(n=17)$ was tested. The methods that were changed from Method 1 were as follows. Microdialysis probes were implanted 3 hours before testing. 2\% $\mathrm{BzCl}$ was used for derivatisation. A new chromatography gradient was used. D2 DA/D2 5-HT $(2.5 \mu \mathrm{L})$ was spiked into each sample/standard to reach a final concentration of $5.56 \mathrm{nM}$. Dialysate concentrations following a $0.0 \mathrm{mg} / \mathrm{kg}$ i.p. dose of MDMA were also determined. Isotopic dilution was used to correct for isotopic 'bleed' from DA and 5-HT into the D2 internal standards. DOPAC and 5-HIAA concentrations were calculated without internal standards.

\section{Results 2}

Unfortunately, attrition rates were high once again. Dialysis issues prevented the collection of samples from 1 of the 17 subjects, but unexpected and uncontrollable issues with the LC-MS that were followed by a lengthy period of maintenance prevented the analysis of dialysate samples from another 9 subjects. Calculated concentrations of DA, 5-HT, DOPAC, and 5-HIAA for a representative subject are shown in Figure 4.8 and are consistent with that shown in previous tests and with previous studies.

Of the data that were able to be analysed correctly, there were still some issues that needed to be addressed. Firstly, calibration curves for DA and DOPAC did not have a large enough range, with the maximum concentrations being 10 and $100 \mathrm{nM}$, respectively. Maximum calculated concentrations of these compounds in dialysate samples were an order of magnitude higher than this and therefore may have been inaccurate. Secondly, the low dialysate concentrations of DA and particularly 5-HT were still difficult to detect, which prevented the accurate quantification of baseline values. Thirdly, there was large variation in the data. The lack of accurate baseline data prevented the transformation of the data to a percentage change from baseline, which might help account for the large between-subject variability and any small variations in probe recovery. The lack of appropriate internal standards for DOPAC and 5HIAA was likely another contributing factor. 

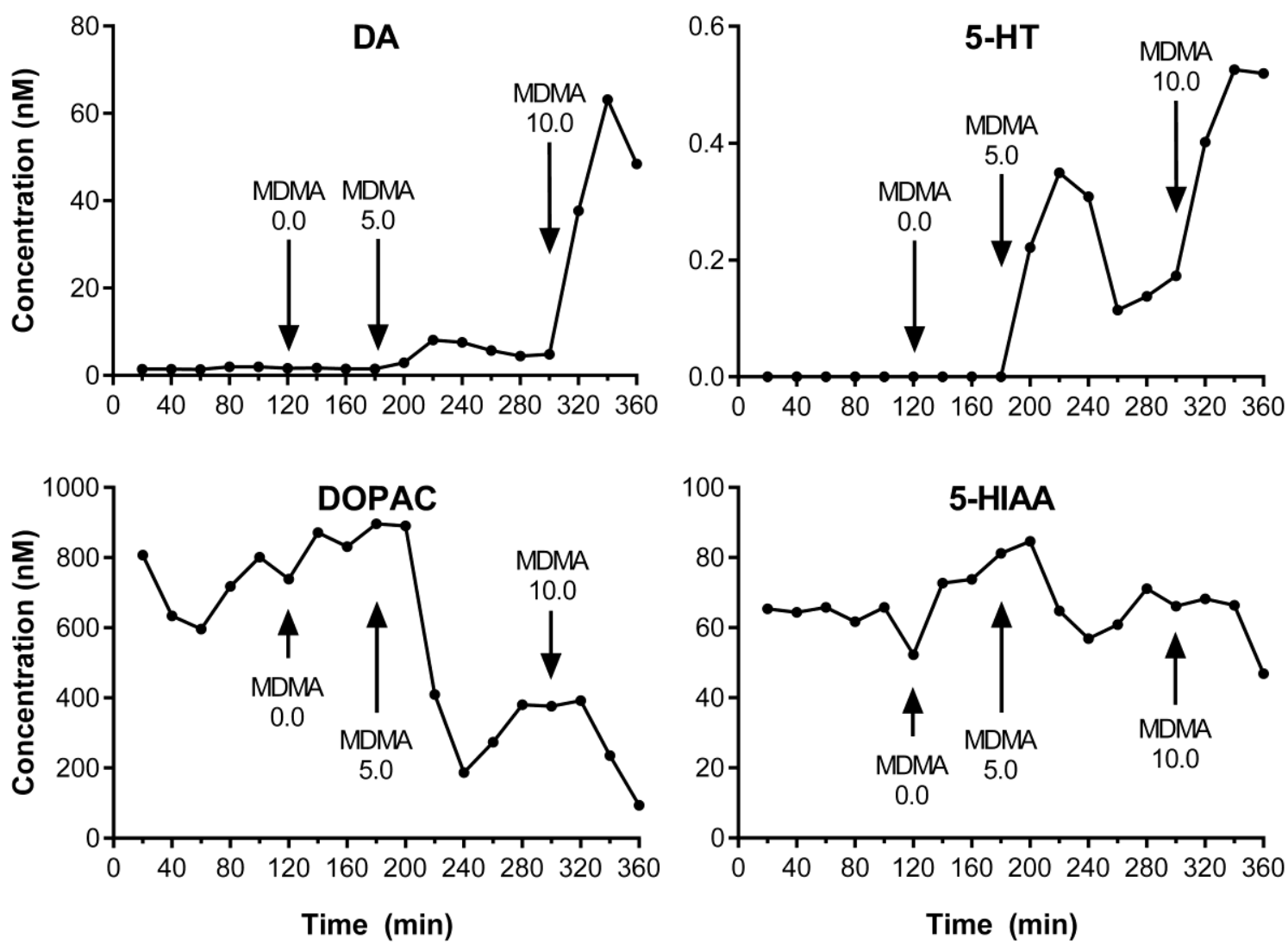

Figure 4.8. Calculated concentrations of dopamine (DA), serotonin (5-HT), DOPAC, and 5HIAA as a function of time/MDMA administration for a representative subject. Microdialysis samples were collected at $20 \mathrm{~min}$ intervals. MDMA 0.0, 5.0, and 10.0 (mg/kg, i.p.) was administered at $120 \mathrm{~min}, 180 \mathrm{~min}$, and $300 \mathrm{~min}$, respectively.

\section{Method development 2}

Several aspects of the procedure were evaluated with the main goal of being able to detect and quantify the low concentrations of DA and 5-HT present in baseline dialysate samples. Unfortunately, further unexpected issues with the LC-MS and another lengthy period of maintenance prevented the collection of data from several tests, which then needed to be repeated.

\section{$\mathrm{BzCl}$ concentration}

Several tests were carried out examining the effect of different $\mathrm{BzCl}$ concentrations $(0.25,0.5,1,2,4,5 \%)$ used for derivatisation on analytical sensitivity. During this time, it was discovered that diluting fresh $\mathrm{BzCl}$ daily greatly decreased variation in the data. This suggests that hydrolysis or evaporation of $\mathrm{BzCl}$ in the solutions used for derivatisation may have 
contributed to the variation of the data in prior work since the same $\mathrm{BzCl}$ solution was previously used for up to 1 week (Bentley, Carter, \& Harris, 1984; V. Gold, Hilton, \& Jefferson, 1954; Hall Jr, 1955; Hudson \& Wardill, 1950).

The effect $\mathrm{BzCl}$ concentration on analytical sensitivity in standards consistently showed that lower concentrations of $\mathrm{BzCl}$ improved sensitivity for DA and particularly 5-HT. Larger peak areas and higher signal to noise ratios $(\mathrm{S} / \mathrm{N})$ were obtained for the same concentration of standard when derivatised with lower concentrations of $\mathrm{BzCl}$ (Figure 4.9). The slopes of calibration curves were also significantly steeper, and the detection limits were lower when using more dilute concentrations of $\mathrm{BzCl}$ (Figure 4.10). In contrast, DOPAC and 5-HIAA became very difficult to detect when using the lowest $\mathrm{BzCl}$ concentrations, eluting very early and often with split peaks (Figure 4.11). These results might be due to differences in sample $\mathrm{pH}$ and insufficient mixing and $\mathrm{pH}$ buffering of the large sample volumes by the mobile phase. Since samples derivatised with lower concentrations of $\mathrm{BzCl}$ have a higher $\mathrm{pH}$, this might impact the retention and ionizability of the more acidic DOPAC and 5-HIAA differently than the basic DA and 5-HT. Changes to the sample or mobile phase buffers were not possible at this stage, and since DA and 5-HT were the primary targets of the analysis, it was decided to use lower concentrations of $\mathrm{BzCl}$ for the improved sensitivity of DA and 5-HT at the expense of being able to quantify 5-HIAA or DOPAC accurately.

Tests examining the effect of different $\mathrm{BzCl}$ concentrations used for derivatisation were also conducted in rats $(n=12)$ on several occasions, first using $1 \% \mathrm{BzCl}$ then using $0.5 \% \mathrm{BzCl}$. Results mirrored those from using standards. The ability to detect baseline DA and 5-HT was improved when using lower $\mathrm{BzCl}$ concentrations, but 5-HIAA and DOPAC were no longer able to be reliably measured when using a $\mathrm{BzCl}$ concentration of $0.5 \%$. Under these conditions, the lowest concentrations of DA were now able to be reliably detected and quantified, but baseline concentrations of 5-HT were still below detection/quantification limits (Figure 4.12). 


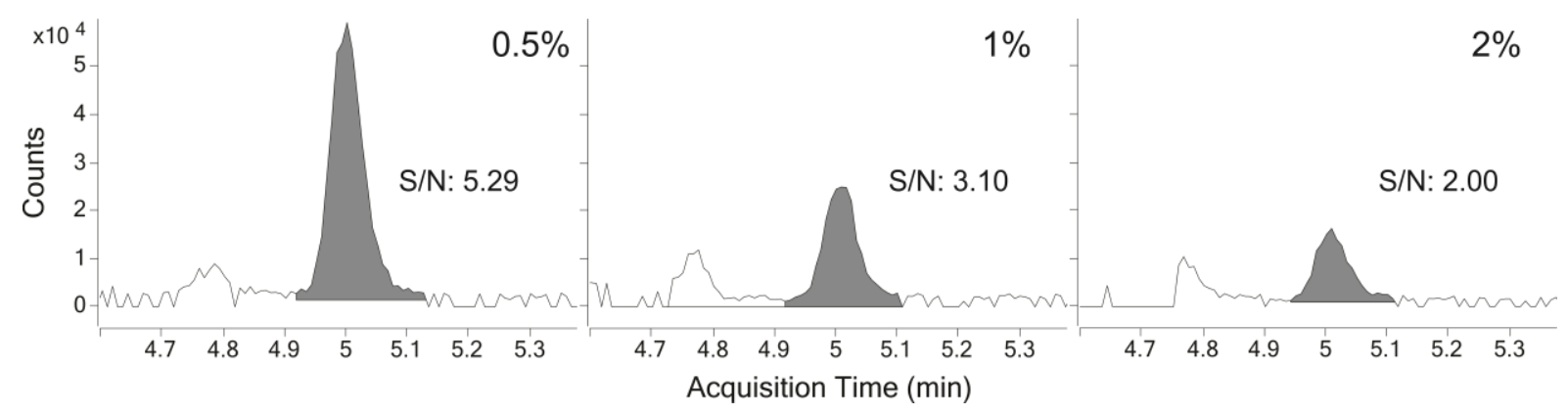

Figure 4.9. Extracted ion chromatograms of serotonin $(385.1547 \mathrm{~m} / \mathrm{z})$ from $0.5 \mathrm{nM}$ external standard following derivatisation with $0.5 \%, 1 \%$, or $2 \% \mathrm{BzCl}$. Signal to noise ratio $(\mathrm{S} / \mathrm{N})$ is shown for each peak.

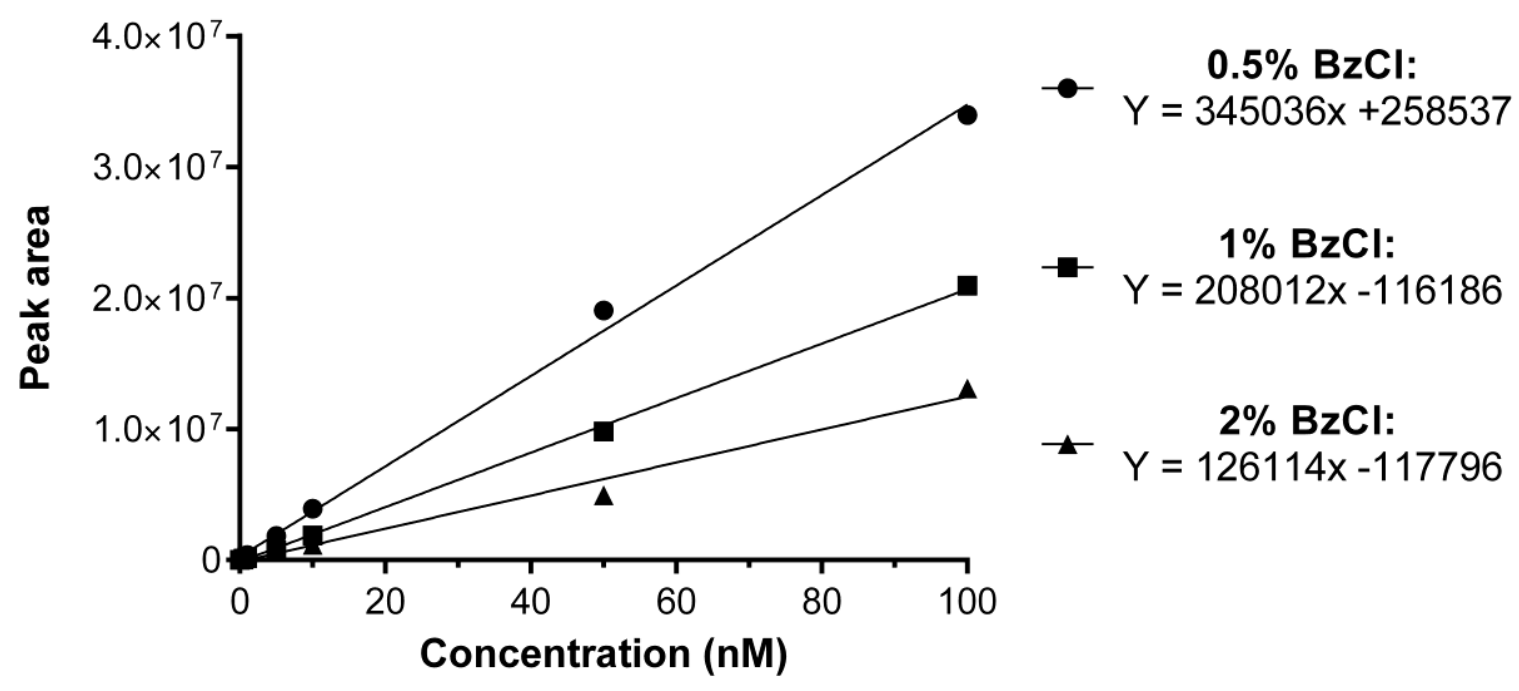

Figure 4.10. 8-point calibration curves for serotonin made from external standards at $0,0.1$, $0.5,1,5,10$, and $50 \mathrm{nM}$ following derivatisation with $0.5 \%(\bullet), 1 \%(\boldsymbol{\bullet})$, or $2 \%(\boldsymbol{\Delta}) \mathrm{BzCl}$. The slope and intercept of each linear calibration curve are displayed in the legends. 


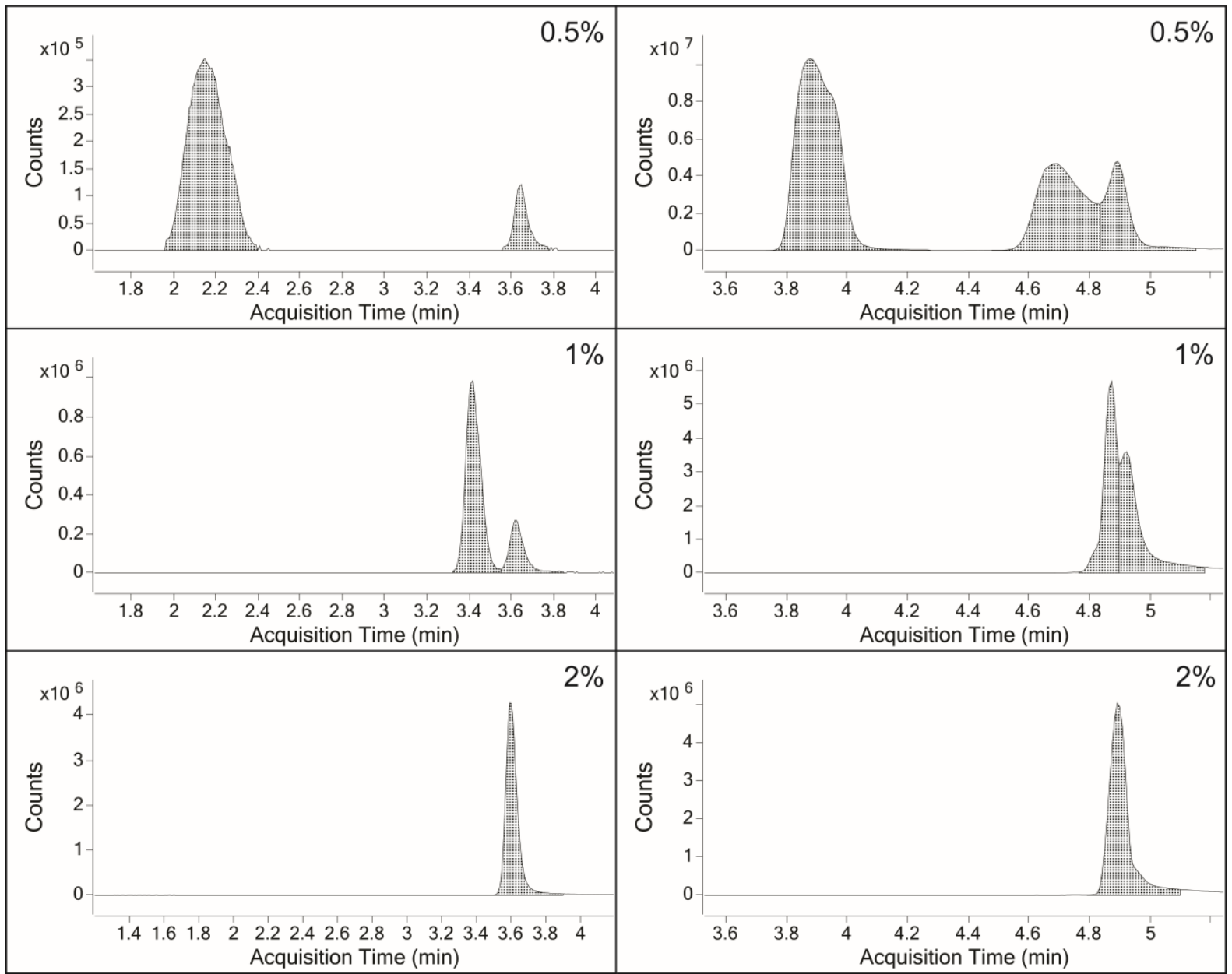

Figure 4.11. Extracted ion chromatograms of 5-HIAA (left) and DOPAC (right) from 1000 $\mathrm{nM}$ external standards following derivatisation with $0.5 \%$ (top), $1 \%$ (middle), or $2 \%$ (bottom) $\mathrm{BzCl}$. 


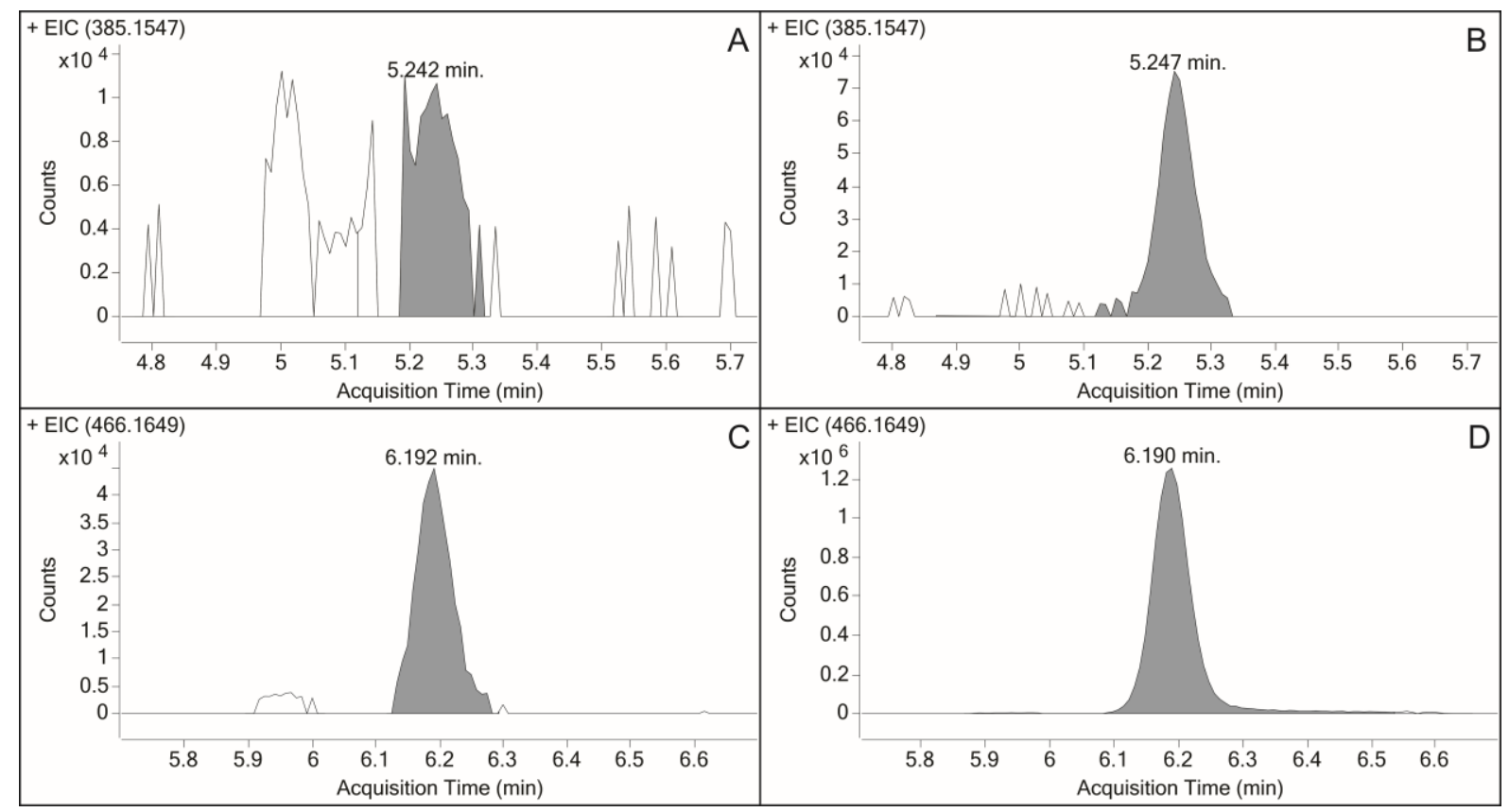

Figure 4.12. Extracted ion chromatograms of derivatised serotonin (panels A and B) and dopamine (panels $\mathrm{C}$ and $\mathrm{D}$ ) in rat brain microdialysate samples during baseline conditions (panels A and C) and following $10 \mathrm{mg} / \mathrm{kg}$ i.p. MDMA (panels B and D). 


\section{LC-MS injection volume}

Several tests were carried out to determine the effect of HPLC injection volume in both external standards and rats $(n=6)$. As expected, increasing the injection volume resulted in a proportional increase in the peak area, but this also increased noise (Table 4.2). The S/N only improved marginally with larger injection volumes. Thus, any gain in detection limits was small and came with the sacrifice of temporal resolution since the microdialysis sampling interval would need to be increased to maintain the same sample volume. Baseline concentrations of 5-HT in dialysate samples were still difficult to detect reliably with even the largest injection volumes.

Table 4.2.

\begin{tabular}{lll}
\multicolumn{3}{c}{ Effect of injection volume } \\
\hline \multicolumn{3}{c}{ 5-HT (1 nM standards) } \\
Injection vol. & Peak Area & $\mathrm{S} / \mathrm{N}$ \\
\hline $10 \mu \mathrm{L}$ & 38771 & 1.25 \\
$30 \mu \mathrm{L}$ & 107230 & 3.45 \\
$60 \mu \mathrm{L}$ & 191553 & 3.80 \\
$100 \mu \mathrm{L}$ & 254905 & 4.16 \\
\hline
\end{tabular}

\section{Microdialysis}

Further increases in sensitivity did not seem achievable without major methodological changes, and thus it was decided to focus on improving analyte recovery during microdialysis. The concentration of the analyte recovered using microdialysis (the relative recovery) only represents a fraction of the concentration present in the extracellular fluid and is influenced by several factors (Chefer et al., 2009; Westerink \& Cremers, 2007). Firstly, the flow rate of the perfusate through the dialysis probe is inversely proportional to relative recovery. At extremely low flow rates, relative recovery can approach $100 \%$, but the volume of sample acquired per unit time will be greatly decreased, and thus the absolute recovery (total amount of analyte per sample) will be low. Conversely, higher flow rates decrease relative recovery while increasing absolute recovery and sample volume. It had already been determined that increasing the sample volume injected into the LC-MS produced marginal increases in the detection limits for 5-HT. It was therefore decided to test a lower flow rate in order to increase relative recovery, 
at the expensive of absolute recovery and sample volume. Halving the current flow rate to 0.5 $\mu \mathrm{L} / \mathrm{min}$ increased relative recovery of 5 -HT standards in vitro from approximately $15 \%$ to $25 \%$.

Another factor that influences the recovery is the probe dialysis membrane surface area. Probes with a greater membrane surface area have greater relative recovery as there is more area for analytes to diffuse across into the perfusate. The relative recovery of several probes from different manufacturers as well as custom-made probes with different size membranes was tested. Probes with a longer membrane ( $3 \mathrm{~mm}$ instead of $2 \mathrm{~mm}$ ) increased relative recovery of the target analytes by approximately $5 \%$ at $1 \mu \mathrm{L} / \mathrm{min}$. Probes with a larger diameter of 0.5 $\mathrm{mm}$ instead of $0.24 \mathrm{~mm}$ further increased recovery, and, when coupled with a $0.5 \mu \mathrm{L} / \mathrm{min}$ flow rate, relative recovery of 5-HT was $45-50 \%$ in vitro. Using these dialysis procedures together with $0.5 \% \mathrm{BzCl}$ derivation, baseline concentrations of 5-HT in microdialysate samples from rats were now reliably above detection limits (Figure 4.13). These dialysis procedures (larger probes, slower flow rates) are similar to those used by Baumann, Clark, and Rothman (2008), who also noted the difficulty in reliably detecting baseline 5-HT samples.

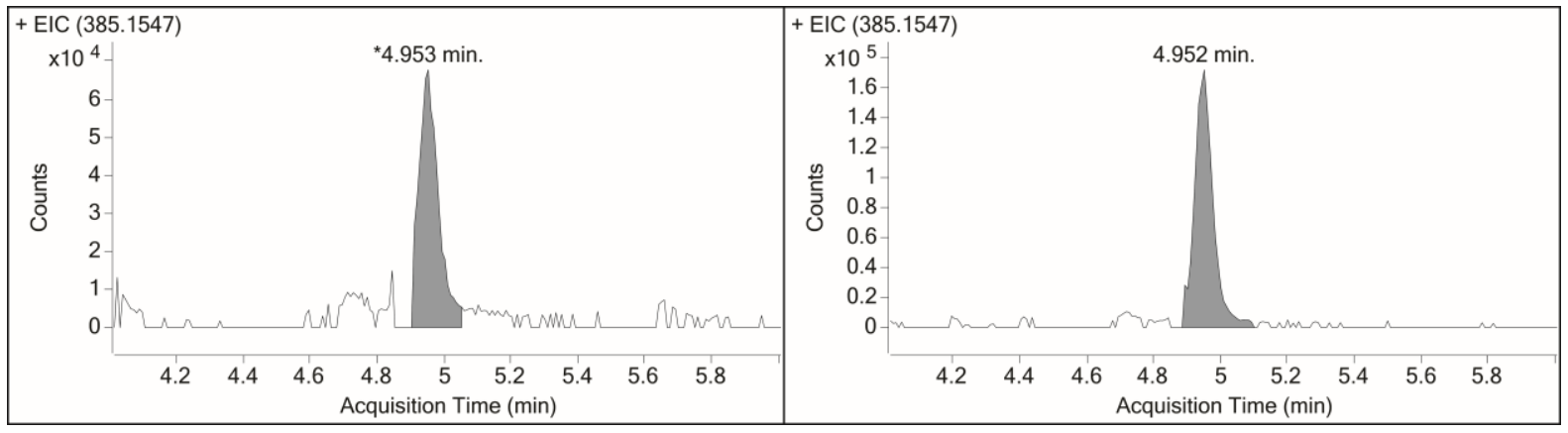

Figure 4.13. Extracted ion chromatograms of derivatised serotonin in rat brain microdialysates during baseline conditions (left) and after $10 \mathrm{mg} / \mathrm{kg}$ i.p. MDMA (right). 
Calculated dialysate concentrations of DA and 5-HT from a test subject are shown in Figure 4.14. Baseline dialysate concentrations of DA and 5-HT were approximately $5 \mathrm{nM}$ and $0.5 \mathrm{nM}$, respectively. DA concentrations peaked at $24 \mathrm{nM}$ (380\% increase) following $5 \mathrm{mg} / \mathrm{kg}$ MDMA and $100 \mathrm{nM}$ (1900\% increase) following $10 \mathrm{mg} / \mathrm{kg}$ MDMA. 5-HT concentrations peaked at $2.7 \mathrm{nM}$ (440\% increase) following both 5 and $10 \mathrm{mg} / \mathrm{kg}$ MDMA. These concentrations and percentage increases are within the range of what is expected based on previous studies (Freezer, Salem, \& Irvine, 2005; Gough et al., 2006; Gudelsky \& Nash, 1996; Hegadoren, Martin-Iverson, \& Baker, 1995; Kankaanpää et al., 1998; Nair \& Gudelsky, 2004; Schmidt, Sullivan, \& Fedayal, 1994).
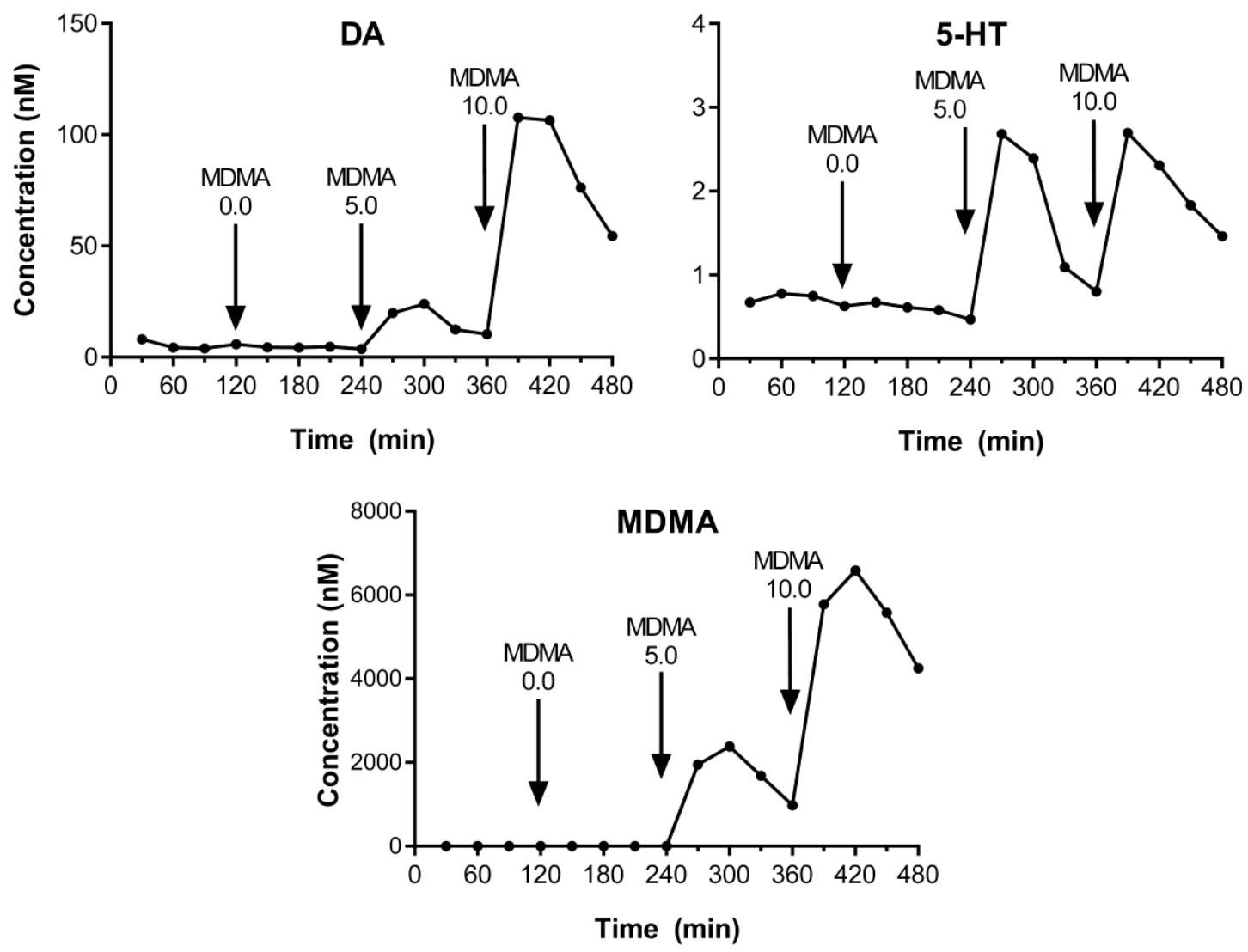

Figure 4.14. Calculated concentrations of dopamine (DA), serotonin (5-HT), and MDMA as a function of time/MDMA administration for a representative test subject. Microdialysis samples were collected at $30 \mathrm{~min}$ intervals. MDMA 0.0, 5.0, and $10.0(\mathrm{mg} / \mathrm{kg}$, i.p.) was administered at $120 \mathrm{~min}, 240 \mathrm{~min}$, and $360 \mathrm{~min}$, respectively. 


\section{Quantification of MDMA}

Calibration curves and external standards were also prepared for MDMA, allowing for the quantification of MDMA in microdialysate samples (Figure 4.14). Following the adjustments to the microdialysis procedure, however, the concentrations of MDMA recovered were very high. Concentrations in dialysate samples following MDMA administration (5 or 10 $\mathrm{mg} / \mathrm{kg}$ i.p.) ranged from $1000-8000 \mathrm{nM}$. These concentrations appeared to saturate the MS detector and were well above the linear range of the calibration curves (Figure 4.15). Dilution of the samples was not viable, while a separate analysis run for MDMA was not possible due to time constraints. Thus, a best estimate for the concentration of MDMA under these conditions was carried out using a non-weighted quadratic calibration curve for MDMA using external standards at 1000, 5000, and $10000 \mathrm{nM}$ (Figure 4.15; Almeida et al., 2002; Moosavi \& Ghassabian, 2018). D2 5-HT was used as the internal standard, and the $\mathrm{R}_{\mathrm{x}} / \mathrm{R}_{\mathrm{y}}$ isotopic dilution for MDMA was calculated manually.

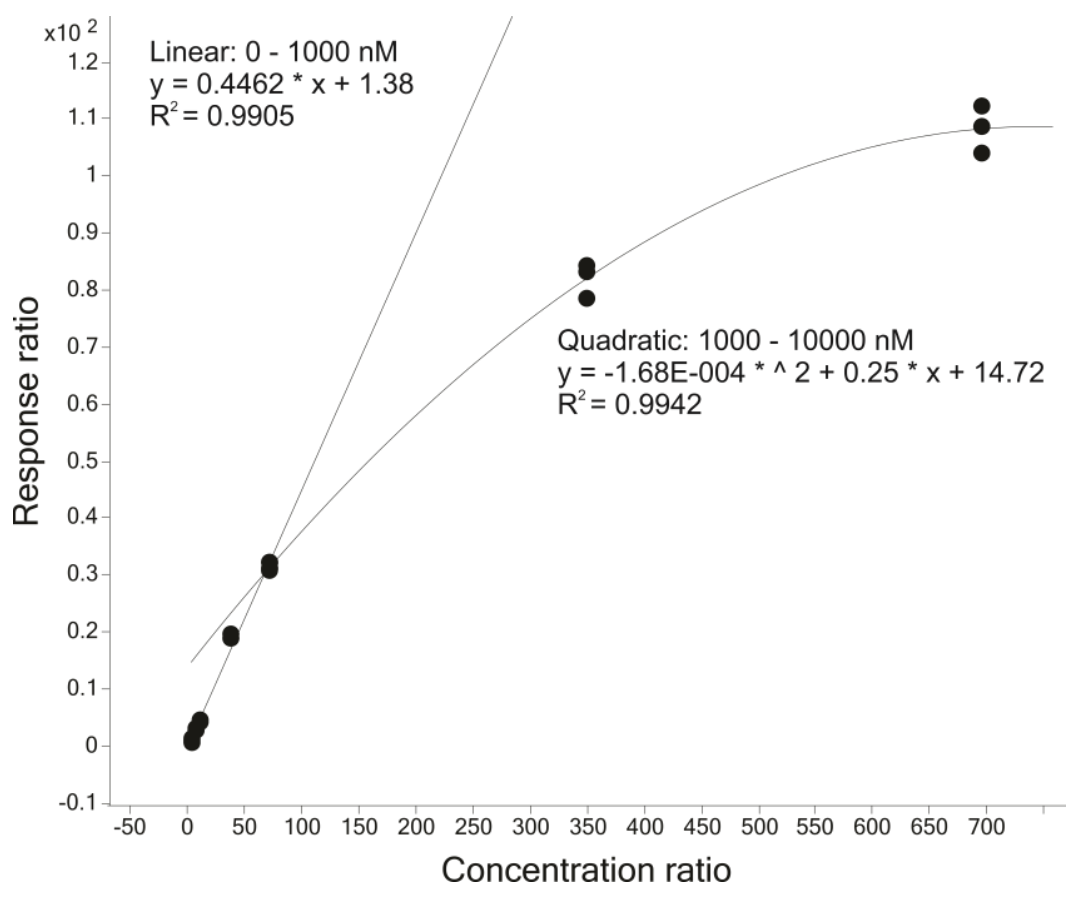

Figure 4.15. Linear and quadratic calibration curves for MDMA made from external standards analysed in triplicate at 0,10,50,100, 500, 1000, 5000, and $10000 \mathrm{nM}$ and D2 5-HT internal standard at $14.3 \mathrm{nM}$. 


\section{Methods 3}

A final sample of rats $(n=24)$ was tested. Dialysis and analytical methods that were changed from Method 2 were as follows. Larger diameter and length guide cannula (9.14.IC, Microbiotech, Sweden) and microdialysis probes (MAB 9.14.3, Microbiotech, Sweden) were used. The membrane area of these probes was $0.5 \mathrm{~mm}$ (diameter) $\times 3 \mathrm{~mm}$ (length). A slower dialysis flow rate of $0.5 \mu \mathrm{L} / \mathrm{min}$ was used, and samples were collected at 30 min intervals. To each $15 \mu \mathrm{L}$ dialysate sample, $2.5 \mu \mathrm{L}$ of internal standard at a higher concentration (100 nM D2 DA/D2 5-HT; final concentration $14.3 \mathrm{nM}), 7.5 \mu \mathrm{L}$ of borate buffer $(100 \mathrm{nM})$, and $7.5 \mu \mathrm{L}$ of freshly diluted $\mathrm{BzCl}$ (0.5\% in acetonitrile) was added, with mixing between each step. The volume of derivatised sample injected by the HPLC was $30 \mu \mathrm{L}$. Calibration curves were made from external standards at $0,0.1,0.5,1,5$, and $10 \mathrm{nM}$ for 5 -HT and $0,1,5,10,50$, and $100 \mathrm{nM}$ for DA. DOPAC and 5-HIAA were no longer quantified. Non-weighted quadratic calibration curves for MDMA were made from 1, 5, and $10 \mu \mathrm{M}$ MDMA prepared in the same way as the other external standards.

\section{MDMA pretreatment and locomotor sensitisation}

The effect of a sensitising regimen of repeated MDMA exposure on locomotor activity and analyte concentration was also determined. Pretreatment with MDMA (0.0 [0.9\% saline] or $10 \mathrm{mg} / \mathrm{kg} /$ day, i.p., for 5 days) was carried out in locomotor activity chambers as previously described in Chapter 3. Following two drug-free days, microdialysis probes were implanted and, 3 hours later, testing began. Dialysate samples were collected at $30 \mathrm{~min}$ intervals for 2 hours prior to and following the administration of ascending doses of MDMA (0.0, 5.0, 10.0 $\mathrm{mg} / \mathrm{kg}$ i.p.). Measurement of locomotor activity was carried out simultaneously as described in Chapter 3, and the data were summed into $30 \mathrm{~min}$ bins to match the microdialysis sampling interval.

\section{Statistical analysis}

Separate 2 (pretreatment: MDMA 0.0 vs $10.0 \mathrm{mg} / \mathrm{kg}$ ) $\times 16$ (time: $30-480 \mathrm{~min}$ ) mixedmeasures ANOVAs were conducted to analyse locomotor activity and analyte concentrations. The average baseline 5-HT and DA concentrations were calculated for each rat, and the percentage change from baseline was also calculated. An independent samples $t$-test was used to compare average baseline analyte concentrations between pretreatment groups. Separate 2 (pretreatment: saline vs $10.0 \mathrm{mg} / \mathrm{kg}$ ) $\times 12$ (time: 150 - $480 \mathrm{~min}$ ) mixed measures ANOVAs were conducted to analyse the DA and 5-HT percentage change data. Greenhouse-Geisser corrections to degrees of freedom (Greenhouse \& Geisser, 1959) were applied when the 
assumption of sphericity was not met, as determined by Mauchly's Test of Sphericity. Significant interactions were followed up with simple main effect analyses. The level of significance for all tests was $p<.05$. All analyses were conducted using SPSS (v25, IBM).

\section{Results 3}

Locomotor and dialysate data (5-HT and MDMA) were obtained from 21 of the 24 rats. Two subjects did not complete the pretreatment phase while one subject had an unacceptable probe placement. Probe placements for these 21 final subjects are shown in Figure 4.16. Quantified dialysate concentrations of DA were only successfully obtained from the first 6 subjects, however. DA data from subsequent analyses were very unreliable, with the DA peaks becoming difficult to detect and quantify accurately, even at high concentrations.

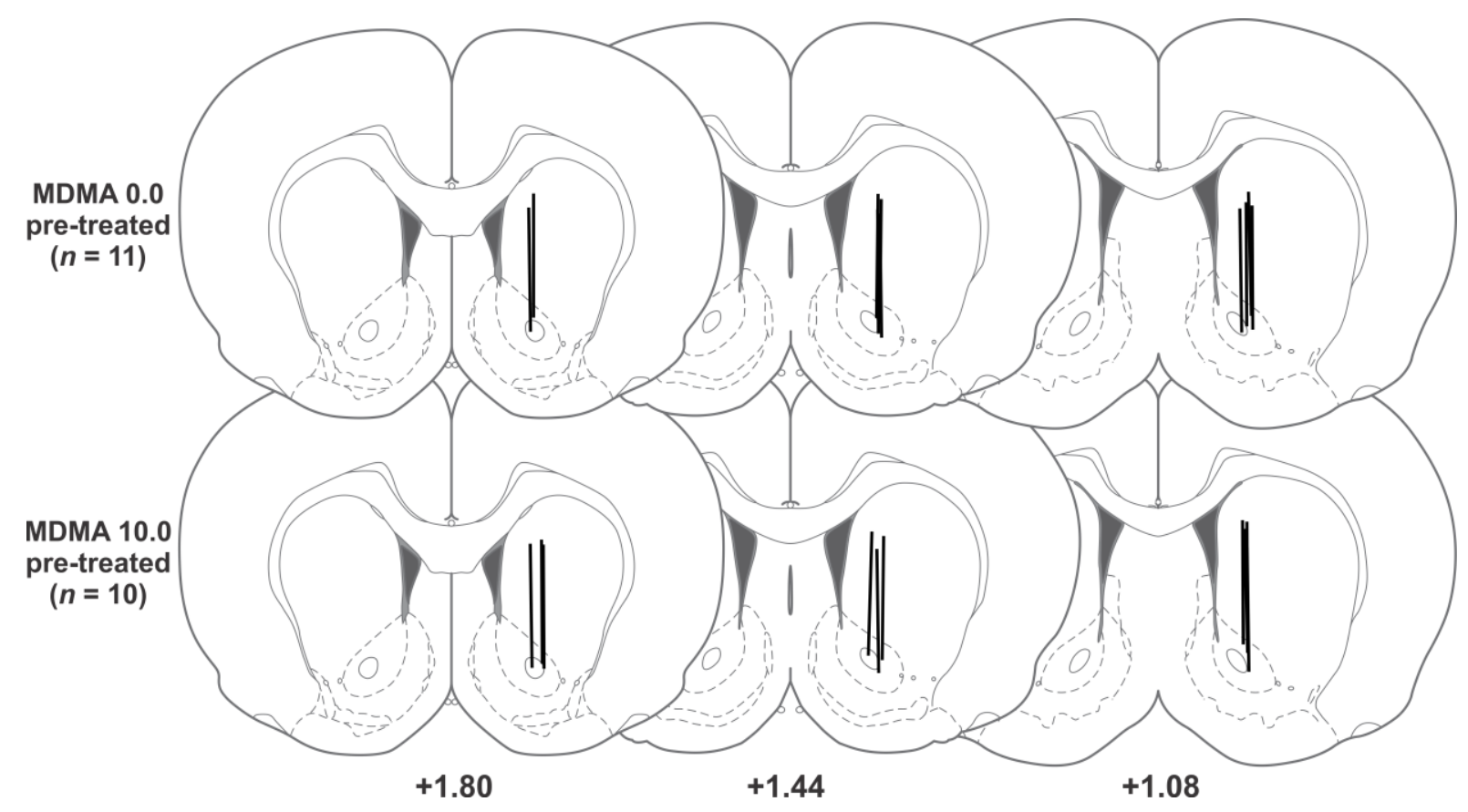

Figure 4.16. Schematic representation (adapted from Paxinos \& Watson, 2005) of microdialysis probe placement in rat medial striatum as a function of MDMA pretreatment $(0.0$ or 10.0). Black bars represent the location of the probe membranes $(0.5 \mathrm{~mm} \times 3 \mathrm{~mm})$ for each rat. Numbers represent the distance from Bregma in $\mathrm{mm}$. Sample sizes are displayed in the legend. 
Figure 4.17 displays locomotor activity as a function of MDMA pretreatment and time/MDMA administration. There was a significant two-way interaction (pretreatment $\times$ time), $F(2.76,52.50)=4.19, p=.012, \eta_{\mathrm{p}}^{2}=.181$. Locomotor activity changed significantly as a function of time for both the saline, $F(15,150)=11.16, p<.001, \eta_{\mathrm{p}}{ }^{2}=.527$, and MDMA $10.0, F(15,135)=8.28, p<.001, \eta_{\mathrm{p}}^{2}=.479$ pretreatment groups. Locomotor activity was significantly greater in the MDMA 10.0 pretreatment group at the $300,330,390,420$, and 450 min time points, $p<.05$.

Figure 4.18 shows mean MDMA concentrations as a function of MDMA pretreatment and time. A two-way ANOVA failed to produce a significant interaction, $p>.05$. A significant main effect of time indicated that MDMA concentrations increased as a function of time/MDMA administration, $F(15,285)=97.66, p<.001, \eta_{\mathrm{p}}{ }^{2}=.837$. There was no main effect of pretreatment indicating that there was no difference in overall MDMA concentrations as a function of MDMA pretreatment, $p>.05$.

Figure 4.19A displays mean 5-HT concentrations as a function of MDMA pretreatment and time. A two-way ANOVA failed to reveal a significant interaction, $p>.05$. A significant main effect of time indicated that 5-HT concentrations increased as a function of time/MDMA administration, $F(3.45,65.56)=90.26, p<.001, \eta_{\mathrm{p}}{ }^{2}=.826$. There was no main effect of pretreatment, indicating that there was no difference in overall 5-HT concentrations as a function of MDMA pretreatment, $p>.05$. The average baseline concentration of 5-HT was marginally higher in the MDMA 10.0 pretreatment group (mean $=0.48 \mathrm{nM}, \mathrm{SD}=0.30$ ) compared to the saline group (mean $=0.34 \mathrm{nM}, \mathrm{SD}=0.15$ ), but this was not significant, $p>$ .05. Figure 4.19B shows the percentage change from baseline in mean 5-HT concentrations as a function of MDMA pretreatment and time. A two-way ANOVA failed to reveal a significant interaction, $p>.05$. A significant main effect of time indicated that the percentage change in 5-HT concentrations increased as a function of time/MDMA administration, $F(1.93,36.60)=$ 38.41, $p<.001, \eta_{\mathrm{p}}{ }^{2}=.669$. A marginal decrease in overall percentage change in 5-HT concentrations was observed in the MDMA 10.0 pretreatment group, but this main effect was not significant, $p>.05$.

Figure 4.20A displays mean DA concentrations as a function of MDMA pretreatment and time for the first 6 subjects, while Figure 4.20B shows the same data converted to a percentage change from baseline. Given the sample sizes were incomplete, no statistical analyses were run on these data, and thus the results are tentative. Data from the first 6 subjects show that DA increased dose-dependently with MDMA administration for both pretreatment 
groups. A trend of increased MDMA-produced increases in DA concentrations was observed in the MDMA 10.0 pretreatment group. The baseline concentrations of DA were also marginally higher in the MDMA 10.0 pretreatment group (mean $=4.95 \mathrm{nM}, \mathrm{SD}=1.31$ ) compared to the saline group (mean $=3.78 \mathrm{nM}, \mathrm{SD}=1.33$ ).

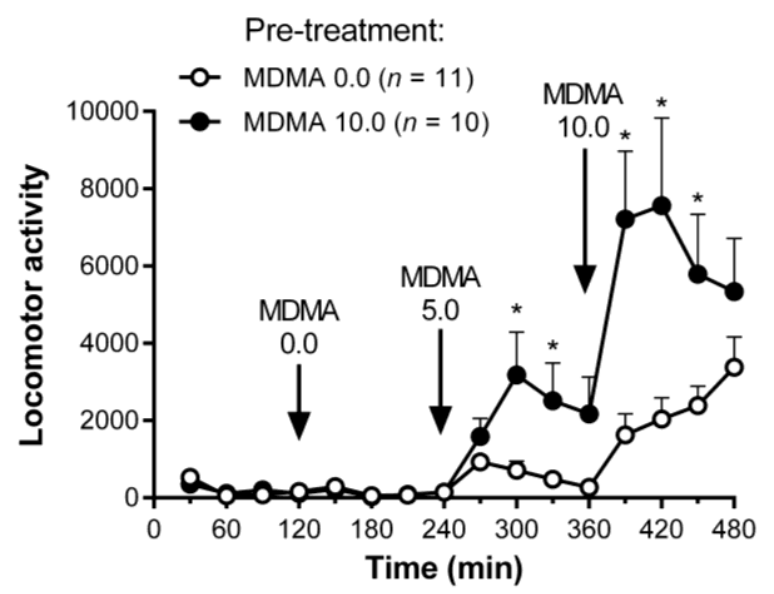

Figure 4.17. Mean locomotor activity (30 min bins) as a function of time/MDMA administration following pretreatment with MDMA 0.0 (०) or MDMA $10.0(\bullet)$. MDMA 0.0, 5.0, and 10.0 (mg/kg, i.p.) was administered at $120 \mathrm{~min}, 240 \mathrm{~min}$, and $360 \mathrm{~min}$, respectively. Error bars represent standard error of the mean. Sample sizes are displayed in the legend. * $p$ $<.05$

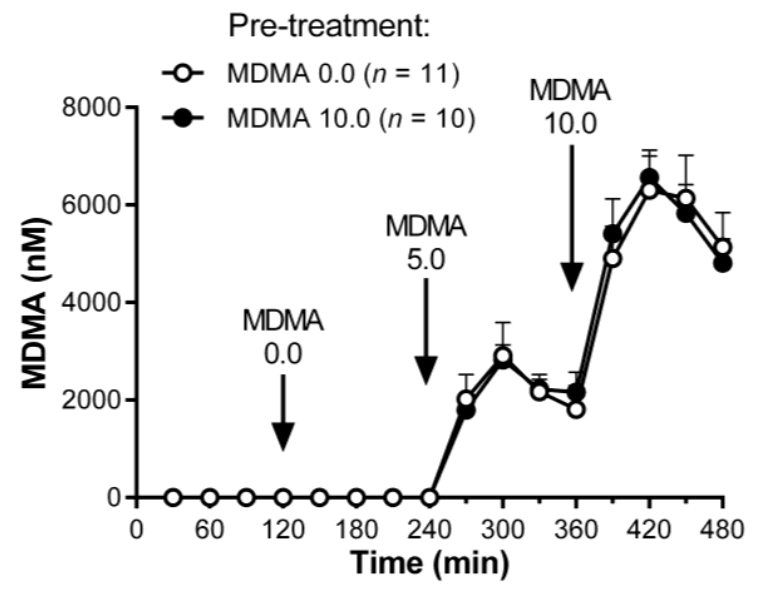

Figure 4.18. Mean MDMA concentrations as a function of time/MDMA administration following pretreatment with MDMA $0.0(\circ)$ or MDMA $10.0(\bullet)$. Microdialysis samples were collected at 30 min intervals. MDMA 0.0, 5.0, and 10.0 (mg/kg, i.p.) was administered at 120 min, $240 \mathrm{~min}$, and $360 \mathrm{~min}$, respectively. Sample sizes are displayed in the legend. 

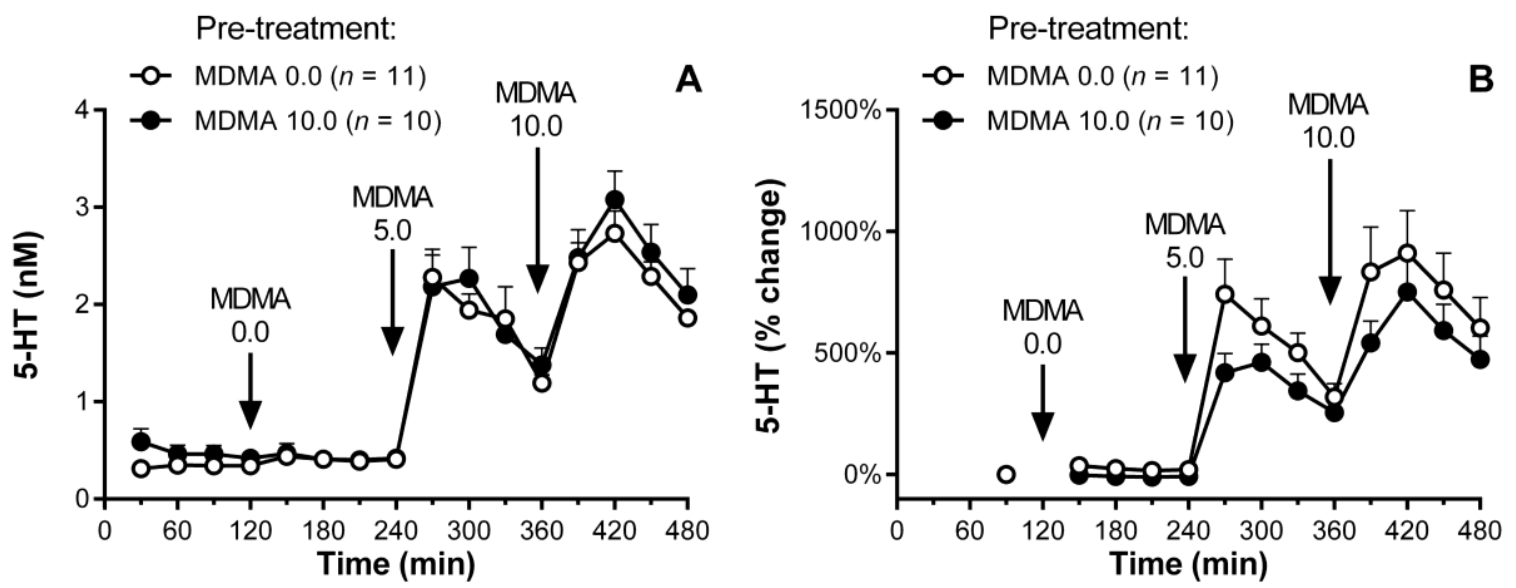

Figure 4.19. Mean serotonin (5-HT) concentrations (A) and mean \% change from baseline (B) as a function of time/MDMA administration following pretreatment with MDMA 0.0 (०) or MDMA $10.0(\bullet)$. Microdialysis samples were collected at 30 min intervals. MDMA 0.0, 5.0, and 10.0 (mg/kg, i.p.) was administered at $120 \mathrm{~min}, 240 \mathrm{~min}$, and $360 \mathrm{~min}$, respectively. Error bars represent standard error of the mean. Sample sizes are displayed in the legend.
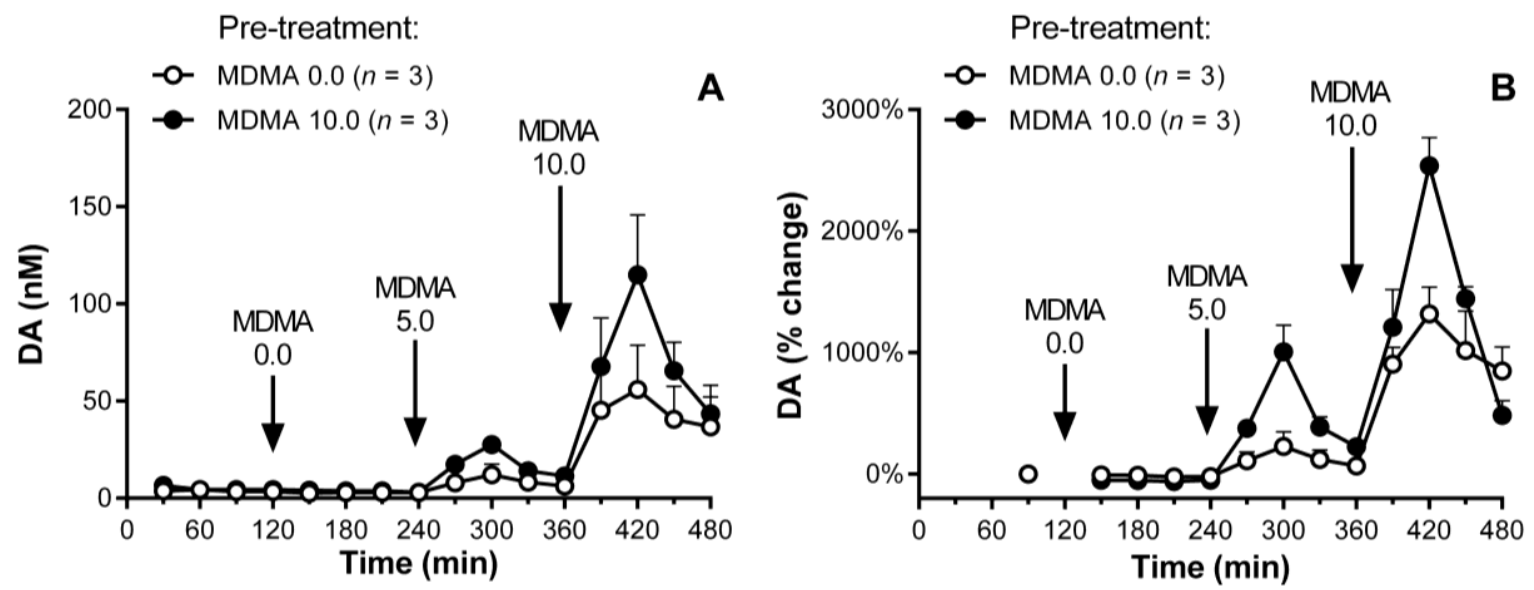

Figure 4.20. Mean dopamine (DA) concentrations (A) and mean \% change from baseline (B) as a function of time/MDMA administration following pretreatment with MDMA 0.0 (०) or MDMA $10.0(\bullet)$. Microdialysis samples were collected at $30 \mathrm{~min}$ intervals. MDMA 0.0, 5.0, and 10.0 (mg/kg, i.p.) was administered at $120 \mathrm{~min}, 240 \mathrm{~min}$, and $360 \mathrm{~min}$, respectively. Error bars represent standard error of the mean. Sample sizes are displayed in the legend. 


\section{Discussion}

In the current study, a procedure for the quantification of extracellular concentrations of 5-HT, DA, and MDMA was developed. Samples were collected using in vivo microdialysis from the medial striatum, derivatised using $\mathrm{BzCl}$, and analysed using HPLC coupled with QTOF ESI-MS. This procedure was then applied to determine neurochemical correlates of sensitised MDMA-produced behaviour following repeated, intermittent exposure.

Acute MDMA administration produced dose-dependent increases in locomotor activity, which was significantly sensitised following repeated exposure, as has been previously shown (Ball, Klein, Plocinski, \& Slack, 2011; Bradbury et al., 2012; Kalivas et al., 1998; van de Wetering \& Schenk, 2017). MDMA-produced locomotor activity was mirrored by dose-dependent increases in extracellular concentrations of MDMA. Correcting for probe recovery, the extracellular concentration of MDMA peaked at approximately $8 \mu \mathrm{M}$ and $17 \mu \mathrm{M}$ following the i.p. administration of 5 and $10 \mathrm{mg} / \mathrm{kg}$ MDMA, respectively, which is consistent with that previously found following similar doses of i.p. MDMA (Esteban et al., 2001). Importantly, MDMA pretreatment had no effect on extracellular concentrations of MDMA following acute administration, suggesting that any behavioural or neurochemical consequences of repeated MDMA exposure in the current study were not due to differences in MDMA uptake.

Acute MDMA exposure also produced dose-dependent increases in extracellular concentrations of 5-HT, although these increases were non-linear and perhaps not what might be expected given a doubling of the MDMA dose. This has also been observed in previous studies (Gough et al., 2006; Kankaanpää et al., 1998; Nair \& Gudelsky, 2004) and can be explained by the pharmacology of MDMA. MDMA is an incredibly potent 5-HT releasing agent, and near-saturated effects of MDMA-produced 5-HT release or 5-HT transporter binding in rat brain tissue slices can be observed with extracellular concentrations of MDMA in the low $\mu \mathrm{M}$ range in vitro (Battaglia, Brooks, et al., 1988; Green, Mechan, Elliott, O’Shea, \& Colado, 2003; Rudnick \& Wall, 1992; Schmidt, Levin, \& Lovenberg, 1987). Given the measured extracellular concentrations of MDMA in the current study were near the top-end of the dose-effect curves from these studies, further substantial increases in 5-HT concentrations following the second, higher dose of MDMA would not be expected. The lack of a further substantial increase in extracellular 5-HT also suggests that other mechanisms are primarily driving the acute MDMA-produced locomotor response, which did increase substantially with the second, higher dose of MDMA. 
In support of this idea, the sensitised MDMA-produced locomotor response following repeated MDMA exposure was not accompanied by significant changes in MDMA-produced extracellular 5-HT. MDMA pretreatment had no effect on the absolute extracellular concentrations of 5-HT following the MDMA challenge. However, there were non-significant trends for higher baseline concentrations of 5-HT and lower MDMA-produced increases in 5HT when calculated as a percentage change from baseline. Previous studies have shown that substantial repeated MDMA exposure resulted in various 5-HTergic deficits such as decreased 5-HT tissue levels, decreased 5-HT transporter densities, and decreased MDMA-produced 5HT release (Battaglia, Yeh, et al., 1988; Battaglia et al., 1987; Commins et al., 1987; Do \& Schenk, 2013; Mayerhofer et al., 2001; O’Hearn et al., 1988; Reveron et al., 2010; Schenk et al., 2007; Shankaran \& Gudelsky, 1999). Repeated exposure to lesser amounts of MDMA have failed to produce such deficits, however (Banks et al., 2008; Do \& Schenk, 2013; Fantegrossi et al., 2004), as was observed in the current study. This suggests that a certain amount of exposure or pattern of exposure is required to produce significant 5-HTergic deficits. The role of 5-HT in the development and expression of MDMA sensitisation has not been well studied. The present findings indicate that changes in MDMA-produced 5-HT release in the medial striatum are not an important mechanism by which repeated MDMA exposure results in behavioural sensitisation.

In contrast to 5-HT, several previous studies have observed increases in DAergic neurotransmission as a function of repeated MDMA exposure and an important role of striatal DAergic mechanisms in the development and expression of MDMA sensitisation has been demonstrated. Repeated exposure to MDMA under conditions that produced behavioural sensitisation also resulted in elevated DA tissue levels (Mayerhofer et al., 2001) and enhanced MDMA-produced DA release (Kalivas et al., 1998). Increases in striatal neuron firing (Ball et al., 2006) and increased dendritic spine density in accumbal MSN (Ball et al., 2009) were also observed following a sensitising regimen of repeated MDMA exposure. Further, administration of the DA $\mathrm{D}_{1}$ receptor antagonist, SCH-23390, prevented the expression of MDMA behavioural sensitisation (Ramos, Goñi-Allo, \& Aguirre, 2004) while co-administration of the $\mathrm{DA} \mathrm{D}_{2}$ antagonist, eticlopride, during pretreatment prevented the development of MDMA behavioural sensitisation (van de Wetering \& Schenk, 2017).

Unfortunately, the DA results from the current study were incomplete and not able to be statistically analysed since reliable data were only obtained from the first 6 subjects of the study. DA data from the other subjects were unreliable; DA peaks were difficult to detect and 
quantify accurately, even at high concentrations. The samples from these subjects were analysed after a long period ( 3 months) of storage at $-80{ }^{\circ} \mathrm{C}$ and following a lengthy period of LC-MS maintenance, which may have impacted the ability to accurately detect and quantify of DA in these samples (Chefer et al., 2009; Suominen et al., 2013).

Nevertheless, the tentative DA results of the current study are consistent with what would be expected based on previous studies. Acute MDMA administration produced dosedependent increases in extracellular concentrations of DA. These increases were also nonlinear, but in contrast to 5-HT, even greater increases on DA were observed than might be expected following a doubling of the MDMA dose. This too has been previously observed by studies that have used similar, relatively high doses of MDMA (Freezer et al., 2005; Gudelsky \& Nash, 1996; Hegadoren et al., 1995; Schmidt et al., 1994). This might be due to dosedependent interactions between 5-HTergic and DAergic neurotransmission since increased 5HT neurotransmission has been shown to be inhibitory to DA release under some conditions (Bankson \& Cunningham, 2001; Czoty et al., 2002; Di Matteo et al., 2008). Following repeated MDMA exposure, the current results would suggest that extracellular concentrations of DA at baseline and following acute-MDMA administration were enhanced. These results, although tentative, are consistent with that found previously using a similar dosing regimen of repeated MDMA exposure (Kalivas et al., 1998) and with the general documented role of DAergic mechanisms in behavioural sensitisation (for reviews see Pierce \& Kalivas, 1997; T. E. Robinson \& Berridge, 1993; Vanderschuren \& Pierce, 2010; Vezina, 2004).

To summarise, in this chapter, a procedure for the sampling and targeted quantification dialysate sample components using microdialysis and LC-MS was developed and validated. This procedure was then employed to determine some of the specific neurochemical correlates of MDMA-induced behavioural sensitisation. The results partially supported predictions in that the MDMA-produced DAergic response appeared to become sensitised with repeated exposure. The MDMA-produced 5-HT response, at least extracellularly, and within the medial striatum, was not impacted, however. These findings add support to the idea that repeated MDMA exposure induces many of the same neuroadaptations that result from repeated exposure to other drugs of abuse (Schenk, 2011). 


\section{CHAPTER 5: UNTARGETED METABOLOMICS}

\section{Introduction}

One of the advantages of MS-based sample analysis that the current research aimed to exploit is the ability to carry out metabolomics (Dettmer, Aronov, \& Hammock, 2007). Metabolomics involves the global, unbiased analysis of the metabolome (all small molecules/metabolites within a biological system) and is typically implemented in order to identify differences between the metabolite profile of a control and test group that might be relevant to specific biological conditions (Fiehn, 2002; Oliver, Winson, Kell, \& Baganz, 1998). Metabolomics is one of the more recent additions to the 'omics' field. Compared to the other 'omics' approaches such as genomics or proteomics, which involve the analysis of the genome and proteome respectively, metabolomics can be more directly relevant to a particular phenotype of interest since metabolites are downstream products of gene transcription and translation (Dettmer et al., 2007; Fiehn, 2002; Patti, Yanes, \& Siuzdak, 2012). The metabolome is much more complex, however, and less well characterised than the genome or proteome (Gromski et al., 2015). The diverse physiochemical properties of metabolites also add to the complexity of metabolomics. No single analytical method is currently able to measure the entire metabolome simultaneously and, thus most metabolomics studies will focus on a certain class of metabolites such as lipids or amino acids (Shulaev, 2006; Zhang, Sun, Wang, Han, \& Wang, 2012).

There are two main approaches in metabolomics; targeted and untargeted (Patti et al., 2012; Shulaev, 2006; Zhou, Xiao, Tuli, \& Ressom, 2012). Targeted approaches are designed to quantitatively measure the absolute concentration of a limited number of known metabolites. Targeted metabolomics analyses are, therefore, partially hypothesis-driven, and are carried out in a similar manner as described in Chapter 4, but with an extensive list of target analytes and external standards (e.g. Song et al., 2012; Wong et al., 2016). The limitation of targeted metabolomics is that the metabolites of interest must be known a priori, which prevents the discovery of novel or genuinely unexpected metabolites that might be relevant. Untargeted approaches, in contrast, are truly hypothesis-generating and aim to semi-quantitatively measure the relative concentration all metabolites within a biological sample.

There are several stages to an LC-MS-based untargeted metabolomics procedure (for reviews see Dettmer et al., 2007; Goodacre et al., 2007; Zhou et al., 2012). The first three stages include sample collection, preparation, and analysis. Various samples such as tissue, plasma, 
urine, or cerebrospinal fluid can be collected and prepared accordingly (e.g. internal standard spike, derivatisation, extraction) for analysis by LC-MS or other means. These procedures are generally designed to be as inclusive as possible so as to provide the largest amount of information on the metabolome. The next stage is data processing. Compared to targeted approaches, where analyte information such as retention time and $\mathrm{m} / \mathrm{z}$ are already known, untargeted data processing is much more complex since the chromatographic peaks of each metabolite need to be correctly distinguished from one another and from chemical or analytical noise automatically. LC-MS data processing can include several steps such as mass detection, chromatogram building, chromatogram deconvolution/peak integration, alignment, and gapfilling, each of which can require considerable optimisation. The next stage is data analysis. Both univariate and multivariate approaches can be used to identify the metabolites that differ between the treatment groups (Bartel, Krumsiek, \& Theis, 2013; Goodacre et al., 2007; Worley \& Powers, 2013). In the final stage, metabolites of interest can be subsequently annotated (tentatively identified) or identified (Sumner et al., 2007). Annotation can be achieved by comparing the exact mass, physicochemical properties, and fragmentation patterns of the metabolite of interest to databases/libraries, while identification can be achieved only with the use of chemical reference standards (Sumner et al., 2007).

The aim of the current research was to expand the targeted findings of the previous chapter and identify novel or unexpected behaviourally relevant compounds that are impacted by repeated MDMA exposure. Untargeted metabolomics analysis was carried out on the microdialysis samples collected from the MDMA vs saline pretreated subjects in Chapter 4. Importantly, these samples were collected over a period of time following the administration of ascending doses of MDMA during which behavioural data (locomotor activity) were also collected. The combination of group and time/dose metabolomics data, as well as behavioural data, makes the current data set unique. This is particularly true within the addiction field, where metabolomics approaches have yet to gain much traction (Ghanbari \& Sumner, 2018; Mussap, Loddo, Fanni, \& Fanos, 2020). Given this unique combination of group, time/dose, and behavioural data, a relatively novel data statistical approach needed to be developed.

Metabolomics studies are typically carried out as a simple two-class comparison (i.e. control vs treated) at a single time point (Bartel et al., 2013; Broadhurst \& Kell, 2007; Gromski et al., 2015). As previously mentioned, univariate tests (multiple t-tests, ANOVAs, etc.) can be carried out to examine class differences. However, due to the extensive number of variables (each metabolite) in an untargeted metabolomics study, this comes with the high risk of making 
Type I errors (Broadhurst \& Kell, 2007). Correction methods such as Bonferroni are typically not appropriate for such large data sets, and even after such corrections, the data can still be difficult to interpret (Broadhurst \& Kell, 2007). Thus, multivariate methods such as principal components analysis (PCA) or partial least squares discriminant analysis (PLS-DA; also known as projection to latent structures) are often used to first summarise the data and identify variables/metabolites of interest prior to any univariate statistics (Gromski et al., 2015; Worley \& Powers, 2013).

PCA reduces the dimensionality of the data, grouping multiple correlated variables/metabolites into a far fewer number of new variables called components that explain the majority of variance in the data set (Jolliffe, 2011; Worley \& Powers, 2013). The first component generated by PCA will always explain the most variance in the data, while the second component will explain the second most variance in the data, and so on. PCA allows for the interpretation of complex multivariate data where patterns, groupings, or outliers can be visualised in 2 or 3 dimensions. If a grouping between different classes is observed and due to one particular component, the contribution that each of the original variables had in generating that component (loadings) can then be examined, and the variables/metabolites that drive the observed grouping can be identified. Because PCA is an unsupervised method that aims to explain the maximum variance in the data, a difference between two classes will only be revealed if the between-class variance exceeds the within-class variance (Worley \& Powers, 2013). This is often not the case with metabolomics data, however, where biological variation, small methodological variations, and variations due to chemical or analytical noise can easily overshadow any experimentally-induced variation in the small number of relevant metabolites (Álvarez-Sánchez, Priego-Capote, \& Castro, 2010; Álvarez-Sánchez, Priego-Capote, \& Luque de Castro, 2010; Worley \& Powers, 2013).

In this situation, supervised methods such as PLS-DA can be much more effective at identifying the drivers of any class differences (Gromski et al., 2015; Wold, Sjöström, \& Eriksson, 2001; Worley \& Powers, 2013). PLS-DA is a regression-based model that similarly reduces the dimensionality of the data into a few components but aims to maximise the covariance of the $X$ variables (i.e. the metabolites) with a designated categorical $Y$ variable that is coded as class membership (e.g. control vs treated). Thus, in regards to metabolomics, PLSDA will aim to maximise the separation between two classes based on any metabolomic differences. Orthogonal PLS-DA (OPLS-DA) is a more recent variant of PLS-DA that includes a orthogonal signal correction (Bylesjö et al., 2006; Trygg \& Wold, 2002; Wold, Antti, 
Lindgren, \& Öhman, 1998) and generates a single component that is predictive of the $Y$ variable while all other components are orthogonal to $Y$ (i.e. independent). This eases the interpretability of the model and essentially splits the data into that which is predictive of $Y$ and representative of between-class variability, and that which is orthogonal to $Y$ and representative of withinclass variability. The variables that load strongly onto the predictive component are, therefore, the variables/metabolites most relevant to the differences between the classes (Galindo-Prieto, Eriksson, \& Trygg, 2014). Variables that load strongly onto orthogonal components may also be of interest for identifying methodological discrepancies or contaminants in the analysis. PCA, PLS-DA, and OPLS-DA have all been used to effectively separate two treatment groups based on their metabolomic profile and identify potential metabolites of interest (e.g. Carrola et al., 2011; H.-J. Kim et al., 2011; Uarrota et al., 2014; X. Wang, Yang, Sun, \& Zhang, 2012).

There is an increasing recent interest in monitoring dynamic changes in the metabolite profile over time in response to various treatments or interventions (Rusilowicz, Dickinson, Charlton, O'Keefe, \& Wilson, 2018; Smilde et al., 2010). While a time-series metabolomics design can be much more powerful and yields much more information, it is also considerably more complex. A single analysis is not capable of fully describing group $\times$ time metabolomics data, but various combinations of methods or models can and have been used effectively (Antti et al., 2002; Boccard \& Rudaz, 2014; Costello \& Martin, 2018; Dai et al., 2016; Galindo-Prieto, Eriksson, \& Trygg, 2015; Guo et al., 2016; Rantalainen et al., 2008; Rusilowicz et al., 2018; Smilde et al., 2010; Williams, Lenz, Rantalainen, \& Wilson, 2006). One such method that is applicable for the current research is the use of standard PLS/OPLS regression models where the $Y$ variable is continuous and representative of time (or other time-related variable of interest). Time-based patterns can then be visualised, and variables that are most predictive and correlated with $Y$ can be identified. These findings can then be used to filter variables for more direct-class based comparisons. Alternatively, time-based models can be generated for both control and treatment groups, which can then be compared in various ways in order to identify differences (Galindo-Prieto et al., 2014, 2015; Wiklund et al., 2007).

The primary goal of the current study was to identify further neurochemical correlates of sensitised MDMA-produced behaviour that were not examined in Chapter 4. A combination of the aforementioned statistical methods including PCA, OPLS, and OPLS-DA, with followup univariate statistics, were used in order to analyse the time-series metabolomics data collected using microdialysis and LC-MS from MDMA pretreated and control rats. The goal of the analysis was to identify metabolites that 1) are relevant to time/MDMA administration, 
2) are relevant to behaviour, and 3) are relevant to MDMA pretreatment. The process of optimising the initial data processing steps are also described in this chapter.

\section{Methods}

\section{Sample collection, preparation, and analysis}

LC-MS data from 336 microdialysis samples collected from the 21 subjects in the final study of Chapter 4 were used. To summarise the study design, subjects were pretreated with either saline $(n=11)$ or MDMA $10 \mathrm{mg} / \mathrm{kg} /$ day $(n=10)$ for 5 days. On the $8^{\text {th }}$ day, microdialysis samples were collected every 30 min over a period of 8 hours (16 samples per rat). During this time, and following an initial 2-hour baseline period, subjects received ascending doses of MDMA $(0.0,5.0,10.0, \mathrm{mg} / \mathrm{kg}$, i.p.) every 2 hours. Locomotor activity was also measured. Microdialysate samples were derivatised with $\mathrm{BzCl}$ and analysed by HPLC coupled with ESI Q-TOF MS detection.

\section{Data processing}

A variety of software packages are capable of processing LC-MS-based metabolomics data. MZmine (version 2.51) was used in the current study as it is one of the most-effective and most cited open-source options designed for this purpose (Z. Li et al., 2018; Myers, Sumner, Li, Barnes, \& Du, 2017a; Pluskal, Castillo, Villar-Briones, \& Orešič, 2010; Samra, 2015). An advantage of MZmine is that each data processing step has a number of available modules/algorithms and each step is also able to be performed independently, which allows for more effective optimisation of the various parameters. The ideal parameters for the current data set were optimised by adjusting these parameters in a step-wise fashion in order to ensure the accurate separation and integration of some known target analytes (i.e. the internal standard [D2 5-HT], 5-HT, and MDMA) while minimising the number of false peaks. The goal was to produce a list of features, where each feature ideally represented a unique ion/metabolite (m/zretention time pair) that has been correctly integrated (peak area). A summary of all final MZmine data processing parameters is shown in Table 5.1, while the process of optimising these parameters is described below. 
Table 5.1.

MZmine data processing parameters

Mass detection (centroid):

Noise level: $10^{4}$

Chromatogram builder (ADAP):

Minimum group size in \# scans: 3

Group intensity threshold: $10^{4}$

Minimum highest intensity: $10^{4}$

$\mathrm{m} / \mathrm{z}$ tolerance: $0.02 \mathrm{~m} / \mathrm{z}$

Deconvolution (local minimum search):

$\mathrm{m} / \mathrm{z}$ centre calculator: median

Chromatographic threshold: $35 \%$

Search minimum in RT range: $0.01 \mathrm{~min}$

Minimum relative height: $5 \%$

Minimum absolute height: $10^{4}$

Minimum ratio of peak top/edge: 2

Peak duration range: $0.02-0.5 \mathrm{~min}$

Isotope peak grouper:

$\mathrm{m} / \mathrm{z}$ tolerance: $0.005 \mathrm{~m} / \mathrm{z}$

RT tolerance: 0.01 min absolute

Monotonic shape: yes

Maximum charge: 2

Representative isotope: lowest $\mathrm{m} / \mathrm{z}$

Alignment (join aligner):

$\mathrm{m} / \mathrm{z}$ tolerance: $0.02 \mathrm{~m} / \mathrm{z}$

Weight for $\mathrm{m} / \mathrm{z}: 3$

RT tolerance: 0.25 min absolute

Weight for RT: 2

Gap-filling (same RT and m/z range):

$\mathrm{m} / \mathrm{z}$ tolerance: $0.01 \mathrm{~m} / \mathrm{z}$ 
Mass detection. The raw data produced by LC-MS contains thousands of successive scans per sample that cover the duration of each analysis (see Figure 1.5 for review). These scans can be visualised as a histogram (mass spectrum) that contain the peak intensity (centroided data) of all the different ions detected and measured by the mass spectrometer. After importing this raw data into MZmine, the mass detection step generates a list of the detected ions/masses and their intensities from each scan for each sample. A noise filter can be applied to exclude ions that do not reach a designated intensity/peak height threshold. This was set at $10^{4}$ following the manual inspection of numerous low-intensity peaks under this threshold that would likely constitute instrumental or chemical noise (Figure 5.1). This threshold was also just below the lowest peak heights of 5-HT in baseline dialysate samples (see Figure 4.13), which is known from previous work in Chapter 4 to be near the lower limit of what is accurately quantifiable with the current sampling and LC-MS methods.

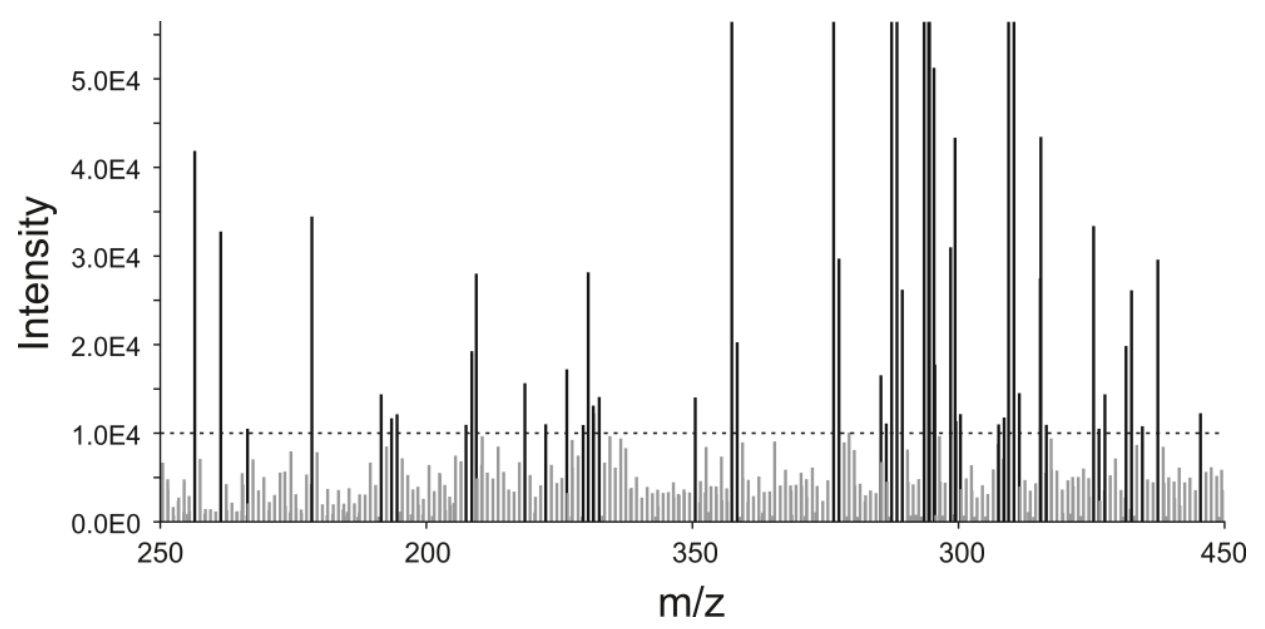

Figure 5.1. An example portion of a raw LC-MS spectrum scan at 5 min retention time showing numerous low-intensity peaks below the $10^{4}$ intensity noise filter (dashed line). 
Chromatogram builder. For each sample, the chromatogram builder step connects the detected masses across each consecutive spectrum scan, building EICs for each. The automated data analysis pipeline (ADAP) chromatogram builder module was used (Myers, Sumner, Li, Barnes, \& Du, 2017b), as this gave the best results. Several settings were adjusted to optimise this step for the current data set. The minimum group size in number of scans was set to 3 and group intensity threshold was set to $10^{4}$. These settings determine the number of consecutive scans a mass must be present and above the intensity threshold to be considered a feature. Higher settings prevented small narrow peaks such as 5-HT from being included, but lower settings included too many extremely narrow peaks that might constitute noise. The minimum highest intensity was set to $10^{4}$ to match the noise threshold in the mass detection step. The $\mathrm{m} / \mathrm{z}$ tolerance setting determines the maximum difference between two $\mathrm{m} / \mathrm{z}$ values to be considered as separate features. Some variation in measured $\mathrm{m} / \mathrm{z}$ values can exist for the same analyte. Thus lower tolerance values would sometimes result in peak splicing and create duplicates of the same ion (Figure 5.2A and B). In contrast, higher values would combine masses that are actually unique together, which is especially problematic when they also have overlapping retention times. This parameter was set to a relatively wide $0.02 \mathrm{~m} / \mathrm{z}$, which was necessary to detect the internal standard in all samples without peak splicing or duplicates (Figure 5.2C).

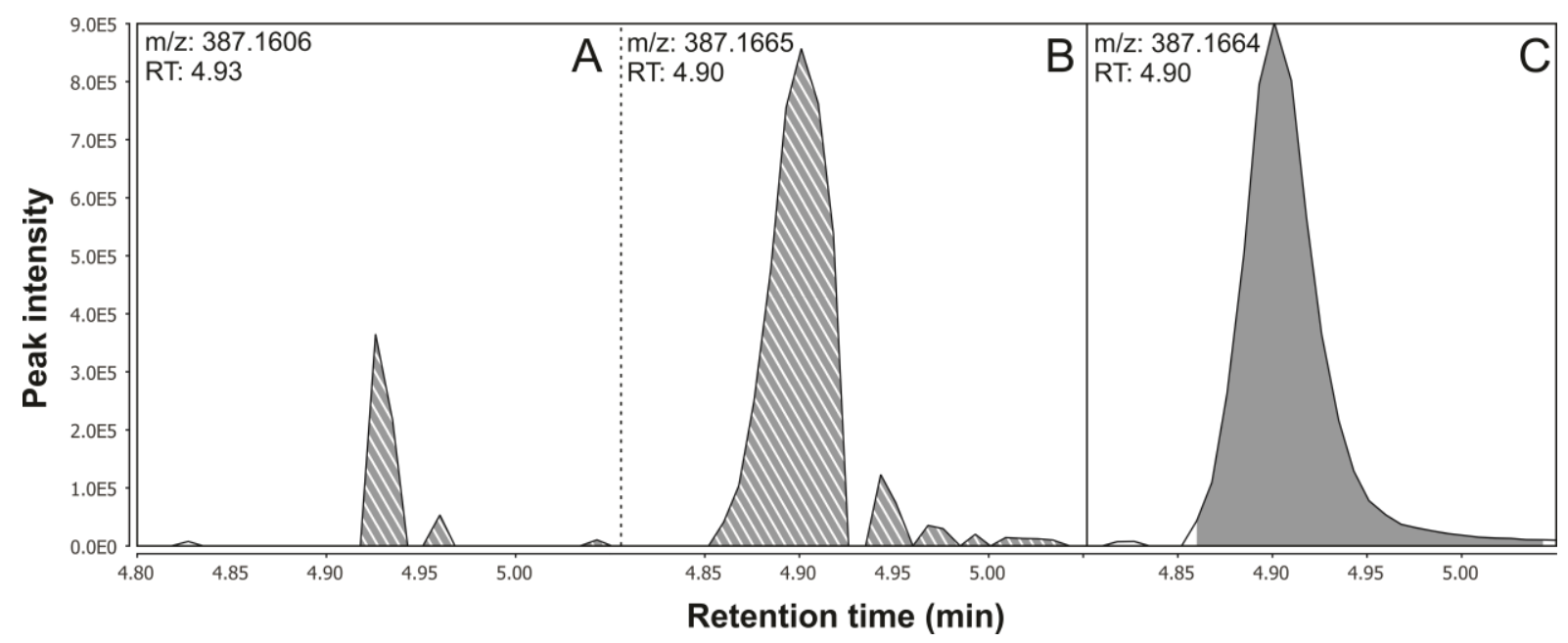

Figure 5.2. Extracted ion chromatograms of the detected derivatised D2 serotonin internal standard peaks using the ADAP chromatogram builder in MZmine. Narrow $\mathrm{m} / \mathrm{z}$ tolerances $(0.005 \mathrm{~m} / \mathrm{z})$ produced duplicate peaks for the internal standard due to peak splicing (A and $\mathrm{B}$ ), which was remedied by widening the tolerance setting to $0.02 \mathrm{~m} / \mathrm{z}(\mathrm{C})$. 
Deconvolution. The deconvolution step detects and integrates peaks within each EIC, producing a feature list for each sample where each feature ideally represents a unique peak/ion. Since many unique metabolites can have a similar $\mathrm{m} / \mathrm{z}$, their peaks will be present within a single EIC, and thus, these peaks need to be separated and individually integrated (Figure 5.3). This is done based on peak retention time, intensity, shape, and other characteristics by various algorithms offered by MZmine. Local minimum search gave the best results with the current data set. The settings for local minimum search were altered in order to correctly detect and integrate the internal standard in as many samples as possible while minimising the number of false peaks. The chromatographic threshold was set at $35 \%$, while the minimum relative height was set at $5 \%$. These settings determine the relative thresholds for reducing noise based on the data points in each EIC. The minimum absolute peak height was set at $10^{4}$ to match previous absolute thresholds in the mass detection and chromatogram builder steps. The search minimum in retention time (RT) range was set at 0.01 minutes and the peak duration range was set to $0.02-0.5$ minutes. These settings determine the minimum retention difference between two peaks to be considered separate features and the range of acceptable peaks lengths/widths, respectively. The minimum ratio of peak top/edge setting was set to 2.0. This setting had the largest impact on the deconvolution results. Lower ratios would generate features from noise (Figure 5.4A), whereas higher values would only detect larger peaks with ideal shapes (Figure 5.4B). The settings chosen were quite conservative in order to minimise the integration of false peaks and ensure accurate peak integration. This did prevent the integration of internal standard peaks from a small number of samples where an HPLC issue caused peak tailing (Figure 5.4C), but this was remedied in a subsequent analytical step.

Isotopic peaks grouper. This step identifies and removes isotopes from each feature list. A relatively narrow $\mathrm{m} / \mathrm{z}$ tolerance of 0.005 was set to avoid removing unique features that may overlap with an isotope. The RT tolerance was set to $0.01 \mathrm{~min}$, which determines the maximum difference a potential isotope can differ from the parent compound. A monotonic shape was required, while the maximum charge to be identified was +2 and the representative isotope was set to be that with the lowest $\mathrm{m} / \mathrm{z}$. Thus, when ions following an isotope pattern were identified, they would be removed, and only the parent compounds with the lowest $\mathrm{m} / \mathrm{z}$ would be kept. This step removed approximately $23 \%$ of features. 


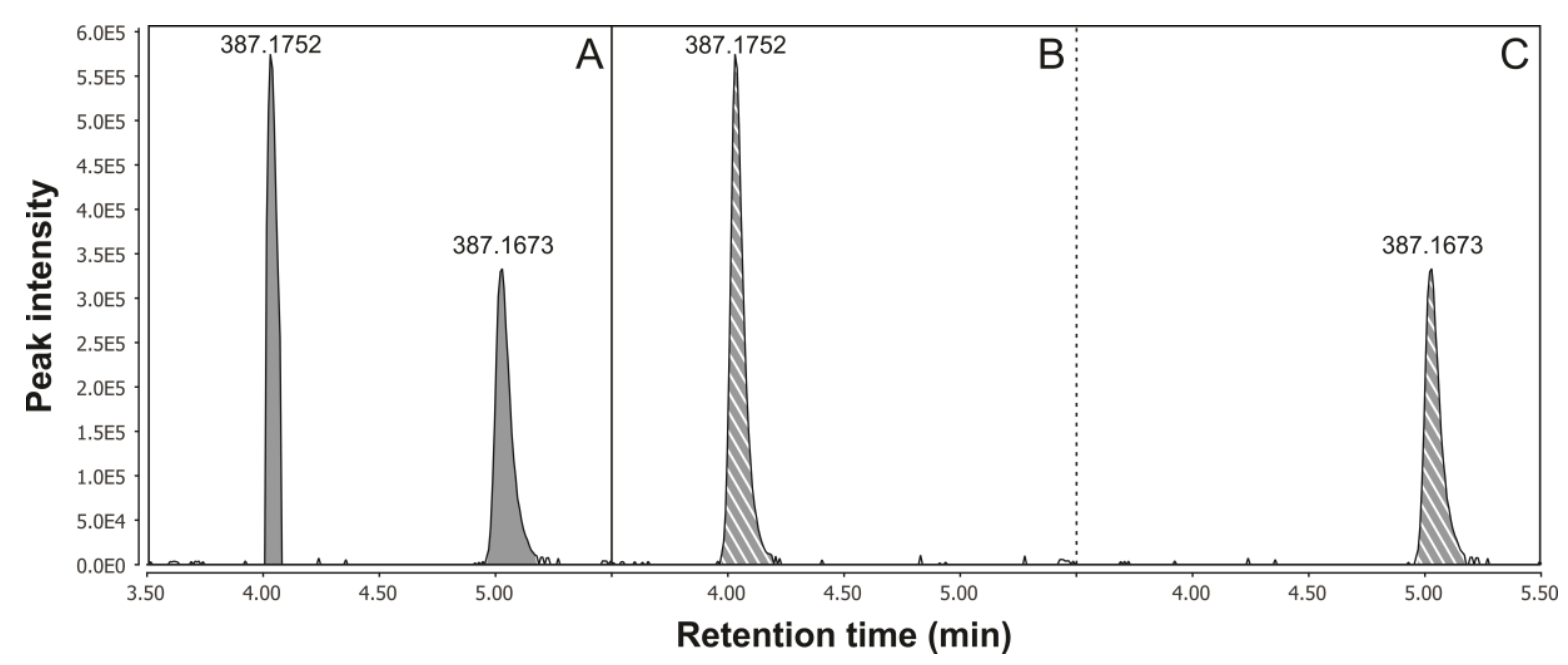

Figure 5.3. Extracted ion chromatograms of $\mathrm{m} / \mathrm{z} 387.1673$ - 387.1752 before (A) and after deconvolution (B and C). Two unique peaks of similar $\mathrm{m} / \mathrm{z}$ were included in a single EIC from the chromatogram builder step (A) but were separated and integrated individually after deconvolution (B and $\mathrm{C})$.

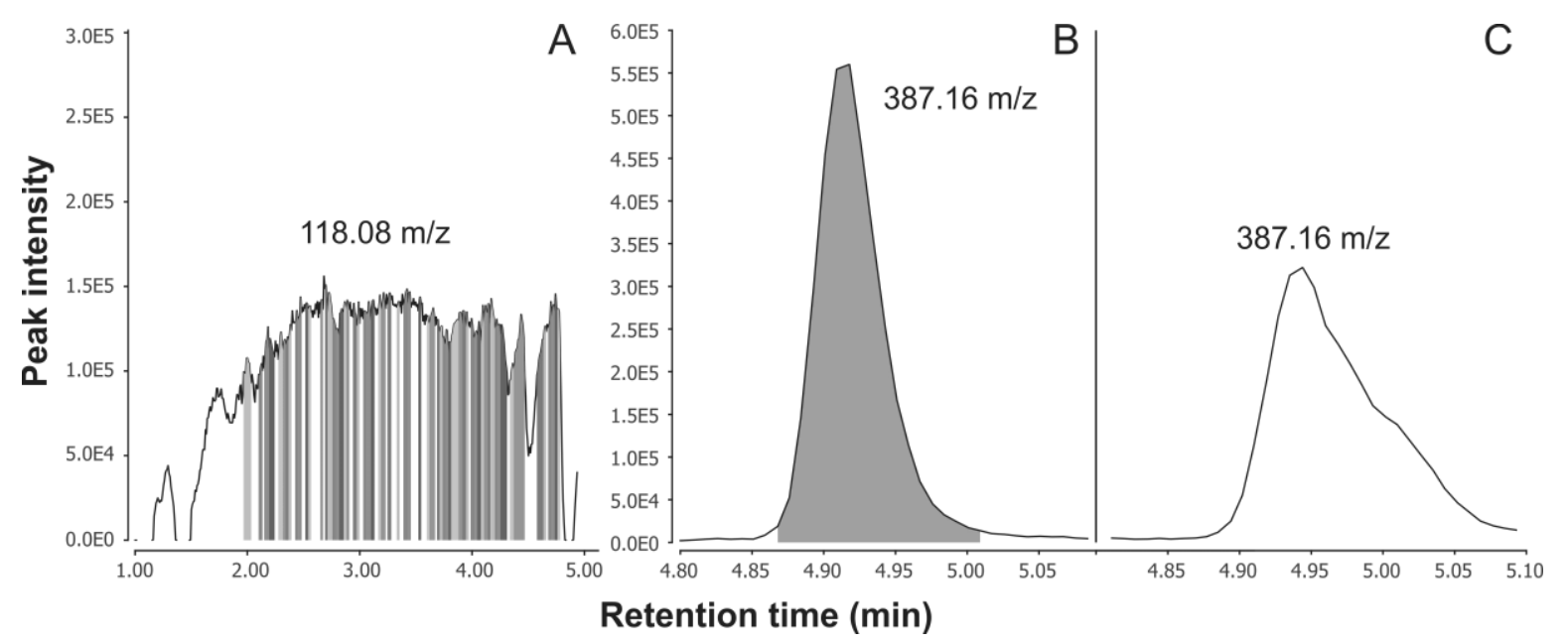

Figure 5.4. Extracted ion chromatogram of $118.08 \mathrm{~m} / \mathrm{z}$ (A) showing MZmine deconvolution results with a low minimum ratio of peak top/edge setting of 0.5 , which generated false peaks (shaded areas) from noise. Extracted ion chromatograms of the derivatised D2 serotonin internal standard (387.16 m/z; B and C) showing MZmine deconvolution results with a higher minimum ratio of peak top/edge setting of 2.00 , which would only integrate peaks with a good shape (B) and not those with tailing or other issues (C). 
Alignment. This step matches all detected features from each of the samples together, generating a single feature list for all samples. The join aligner method was found to be the most effective. The $\mathrm{m} / \mathrm{z}$ tolerance was set to $0.02 \mathrm{~m} / \mathrm{z}$ in order to prevent the generation of duplicate features due to variations in peak $\mathrm{m} / \mathrm{z}$ between samples. The RT tolerance was set to $0.25 \mathrm{~min}$, which was large enough to cover the maximum variation in peak retention times between samples. A weighting of 3 and 2 were given to the $\mathrm{m} / \mathrm{z}$ and retention time settings, respectively. Like the tolerance settings in previous steps, narrower tolerances would result in duplicate features while wider tolerances would combine unique features together. The settings used were the narrowest tolerances required in order to correctly align the internal standard and other targets across all samples without any duplicates.

Gap-filling. This step detects and integrates features that were not detected in every sample. For example, if feature A was only detected in samples 1 and 3, but not 2, gap-filling would re-analyse sample 2 in an attempt to detect and integrate the missing peak. The same RT and $\mathrm{m} / \mathrm{z}$ range method was used with an $\mathrm{m} / \mathrm{z}$ tolerance of $0.02 \mathrm{~m} / \mathrm{z}$. This setting was the minimum required in order to correctly integrate the missing internal standard peaks from the deconvolution step (Figure 5.4C) without any peak splicing or merging. Gap-filing does not have a noise filter threshold, however. Thus, many of the gap-filled peaks were very small and may constitute noise. These features were removed in a subsequent filtering step.

Validation. The current aligned and gap-filled feature list contained 7423 features. To validate the procedure, the peak areas of benzoylated D2 5-HT, 5-HT, and MDMA, as detected and integrated by MZmine in all 336 samples, were compared to the peak areas of these analytes as determined by the targeted integration of these analytes as carried out in Chapter 4. Viewing this data as on a scatter plot revealed a group of outliers where 5-HT was incorrectly integrated (merged peaks) in a small group of samples. This was resolved by reducing the $\mathrm{m} / \mathrm{z}$ tolerance in the gap-filling step to $0.01 \mathrm{~m} / \mathrm{z}$. The final methods resulted in a linear relationship between the peak areas of the three compounds, as detected by each method (D2 5-HT: $\mathrm{R}^{2}=$ 0.983, 5-HT: $\mathrm{R}^{2}=0.988$, MDMA: $\left.\mathrm{R}^{2}=0.999\right)$.

Filtering and final feature list. In order to filter out bad peaks, noise, and other nonrelevant features, features that were not detected in at least $50 \%$ of samples were removed. Further, features that were initially detected in a small number of samples, but subsequently gap-filled in many other samples were removed, as determined by a detected:gap-filled ratio of 0.33 . After filtering, the final feature list contained 737 features. 


\section{Data analysis}

All processed metabolomics data were normalised to the internal standard (peak area), log-transformed in order to achieve a normal distribution, and Pareto scaled so as to avoid highly abundant features such as MDMA from dominating the statistical models, as is recommended for MS metabolomics data (Livera et al., 2015; Sysi-Aho, Katajamaa, Yetukuri, \& Orešič, 2007; van den Berg, Hoefsloot, Westerhuis, Smilde, \& van der Werf, 2006; Veselkov et al., 2011; Wheelock \& Wheelock, 2013). The goal of the data analysis was to identify metabolites of interest based on three criteria. 1) The metabolite should be relevant to MDMA administration, and its relative concentration should change in a dose-dependent manner. 2) The metabolite should be relevant to behaviour, and its concentration should correlate in some way with locomotor activity. 3) The metabolite should be relevant to MDMA pretreatment, and its concentration should change in some way as a function of repeated MDMA exposure. This was achieved by using a combination of PCA, OPLS, and OPLS-DA models as well as follow-up univariates statistics.

First, an overview of the data was provided by PCA, which was carried out using all 737 variables (detected features/metabolites) and all 336 samples (observations). PCA data was evaluated by examining score plots for potential groupings and component loadings to identify relevant variables.

Criteria 1 was assessed by two separate OPLS regression models with the $Y$ variable as MDMA response (peak area) on all samples collected from either the saline or MDMA pretreated rats. Score plots were examined to determine grouping based on acute MDMA administration (baseline, $0.0,5.0,10.0 \mathrm{mg} / \mathrm{kg}$ ). Loading plots were examined, and a ranked list of variables with a predictive Variable Importance in Projection (VIP) score of $>1$ (GalindoPrieto et al., 2014, 2015) was generated, which represented the variables/metabolites that were most correlated and predictive of the MDMA response.

Criteria 2 was similarly assessed by two separate OPLS regression models, but with the $Y$ variable as locomotor activity for all samples collected from either the saline or MDMA pretreated rats. Score plots were examined to determine grouping base on acute MDMA administration (baseline, 0.0, 5.0, $10.0 \mathrm{mg} / \mathrm{kg}$ ). Loading plots were examined, and a ranked list of variables with a predictive VIP score of $>1$ was generated, which represented the metabolites that were most correlated and predictive of locomotor activity.

Criteria 3 was assessed in two ways. First, was by examining Shared and Unique Structures (SUS) plots, where the predictive loadings of variables from two models (i.e. saline 
vs MDMA pretreated) are plotted against each other (Wiklund et al., 2007). SUS plots were generated for the pair of OPLS models generated for assessing criteria 1 (MDMA response) and for the pair of OPLS models generated for assessing criteria 2 (locomotor activity). Variables that were differentially predictive of the MDMA response or locomotor activity in either pretreatment group were noted. Second, three separate OPLS-DA models with dummy $Y$ variables coded as pretreatment group (saline vs MDMA) were generated for the baseline/0.0 samples, the MDMA 5.0 samples, and the MDMA 10.0 samples. Score plots were examined to determine grouping based on pretreatment group. Ranked lists of variables/metabolites that were relevant to the separation of saline and MDMA pretreated rats from these three models were generated based on predictive VIP scores that were $>1$.

Finally, to determine variables that meet all three criteria, the variables that were relevant to class separation (criteria 3) were filtered by those that were also relevant to the MDMA response (criteria 1) and locomotor activity (criteria 2). Univariate statistics ( $t$-tests, ANOVAs) on remaining variables of interest were carried out. All multivariate analyses were carried out using the Soft Independent Modelling of Class Analogies software (SIMCA; v16, Umetrics) while univariate analyses were carried out using SPSS (v25, IBM). All multivariate models were autofitted by SIMCA, and the default K-fold cross-validation procedure was performed. The quality of the model was determined by examining the cumulative $\mathrm{R}^{2} X$ (close to 1 ), which represents the explained variation in $X, \mathrm{R}^{2} Y$ (close to 1 ), which represents the explained variation in $Y$, and $\mathrm{Q}^{2}$ (above 0.5 ), which represents the predicted variation in $Y$ (Szymańska, Saccenti, Smilde, \& Westerhuis, 2012; Triba et al., 2015; Wheelock \& Wheelock, 2013). The level of significance for all tests was $p<.05$.

\section{Metabolite annotation and MS/MS}

The variables/metabolites of interest were tentatively annotated by examining the mass of all observable adduct ions $\left(\mathrm{M}+\mathrm{H}, \mathrm{M}+\mathrm{Na}, \mathrm{M}+\mathrm{NH}_{4}\right.$ etc. $)$ and any fragments generated by insource fragmentation (Gabelica \& Pauw, 2005; Sumner et al., 2007; Xu, Lu, \& Rabinowitz, 2015). Because most metabolites were derivatised, the mass of suspected metabolites following derivatisation was calculated and were compared to the results from two studies that have previously characterised the $\mathrm{BzCl}$ derivatisation and primary MS/MS fragments of several metabolites from microdialysis samples (Song et al., 2012; Wong et al., 2016).

To provide a more confident annotation (Sumner et al., 2007), targeted MS/MS was carried out on selected metabolites of interest and fragmentation patterns were compared to the METLIN database (C. A. Smith et al., 2005). Following the initial LC-MS analysis, remaining 
volumes of microdialysate samples were pooled across all subjects into baseline, MDMA 0.0, MDMA 5.0, and MDMA 10.0 fractions. Triplicate $10 \mu \mathrm{L}$ injections from each pooled sample were then injected using the same HPLC procedure as described in Chapter 4. Targeted Q-TOF ESI-MS/MS was carried out with the following parameters: positive ion mode; gas temperature, $275^{\circ} \mathrm{C}$; gas flow, $8 \mathrm{~L} / \mathrm{m}$; nebulizer, 30 psi; capillary voltage, $2750 \mathrm{~V}$; nozzle voltage, $0 \mathrm{~V}$; fragmentor voltage, $130 \mathrm{~V}$; acquisition rate, 2 spectra/s; mass range, m/z 50 500; collision-induced dissociation energy, 10, 20, and $40 \mathrm{eV}$.

\section{Results}

An overview of the data was provided by PCA on the metabolite profile (737 features/variables) from all 336 samples, $\mathrm{R}^{2} X=0.90, \mathrm{Q}^{2}=0.85$. The PCA was visualised by plotting the component scores of each individual sample. Figure 5.5A shows the scores plot of each sample for component 1 versus component 2. Some outliers were present as determined by the ellipse representing the $95 \%$ confidence interval for Hotelling's $T 2$ test. Samples that cluster together represent those with a similar metabolomic profile. No grouping of the data was observed based on class/MDMA pretreatment group or time/acute MDMA dose (colourcoding not shown in graph). The most apparent grouping was based on individual subjects/analysis batches, suggesting the primary variation in the data is uninduced and stems from sampling, preparation, or analytical variation. Figure 5.5B shows the scores plot of each sample for component 1 versus component 3. Some outliers were also present in this plot. Grouping was observed based on time/acute MDMA dose, with MDMA 5.0 and 10.0 samples being separated from the baseline and MDMA 0.0 samples by the $3^{\text {rd }}$ component. Figure 5.5C is a histogram displaying the loading of each variable (i.e. metabolite) on component 3 as a function of retention time. Several variables had a strong negative loading on component 3 and were driving the observed separation based on MDMA dose. These included MDMA itself (and several adducts/fragments), 5-HT, and what was suspected to be 3-methoxytyramine (3MT), MDA, HHMA, HMMA, and HMA (see Figure 5.12 for a summary of the various variables/metabolites of interest and explanatory examples referred to throughout this results section). 


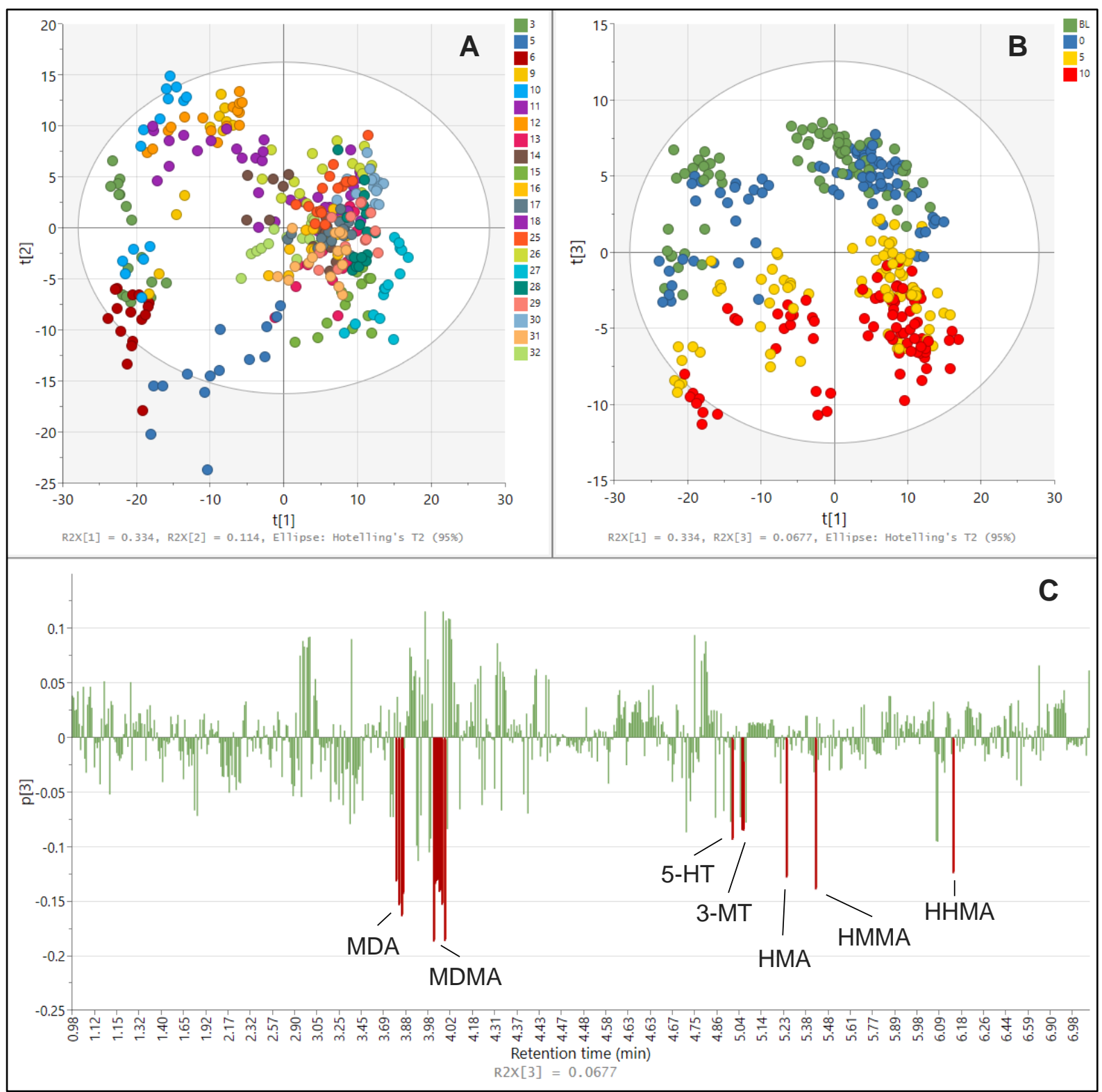

Figure 5.5. Results of principal components analysis on the metabolite profile of all 336 microdialysis samples. A) Score plot of component 1 versus 2 showing colour-coded groupings based on subject/analytical batch. B) Score plot of component 1 versus 3 showing colour-coded grouping based on acute MDMA dose (baseline [BL], 0.0, 5.0, $10.0 \mathrm{mg} / \mathrm{kg}$ ). C) Histogram showing variable loadings for component 3 as a function of retention time. Various annotated metabolites of interest are labelled. 
Identification of metabolites that were relevant to MDMA administration (criteria 1) was achieved by separate OPLS models for each treatment group with the $Y$ variables as the measured MDMA response, saline: $\mathrm{R}^{2} Y=0.98, \mathrm{Q}^{2}=0.97$; MDMA: $\mathrm{R}^{2} Y=0.98, \mathrm{Q}^{2}=0.96$. Figure 5.6 shows the score plots for these models with $\mathrm{t}[1]$ being the predictive component and $\mathrm{t}[2]$ being the orthogonal component plotted on the $\mathrm{x}$ - and y-axis, respectively. Grouping of samples was observed based on acute dose of MDMA. Figure 5.7 shows the loading plots of these two OPLS models with each variable plotted as a function of their predictive (p[1]) and orthogonal (pso[1]) loadings on the $\mathrm{x}$ - and y-axis, respectively. Variables/metabolites that are plotted high on the $\mathrm{x}$-axis represent variables that were most positively predictive of the MDMA response such as the adducts, fragments, and metabolites of MDMA, 5-HT, and 3MT. Variables that are plotted low on the $\mathrm{x}$-axis represent variables that were most negatively predictive of the MDMA response such as $387.17 \mathrm{~m} / \mathrm{z}$. Variables plotted high or low on the $\mathrm{y}-$ axis represent variables that had high variability that was orthogonal to the MDMA response and thus likely not of interest, such as $307.17 \mathrm{~m} / \mathrm{z}$. DOPAC had both a moderately negative predictive and orthogonal loading. Variables plotted near the centre represent variables with little variation in any direction and were similarly not of interest, such as $301.14 \mathrm{~m} / \mathrm{z} .155$ variables had a predictive VIP score of $>1$.

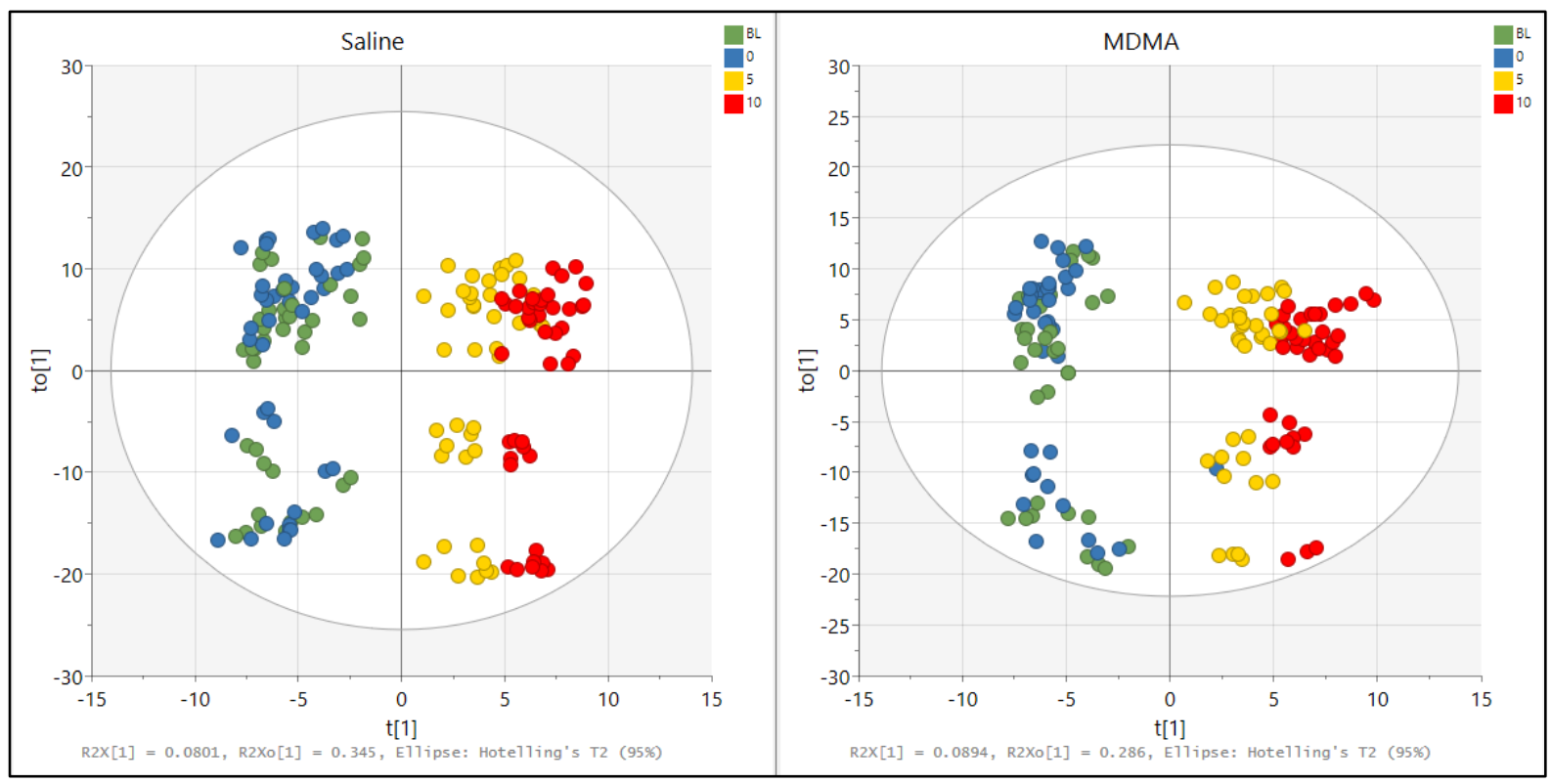

Figure 5.6. Score plots generated from two OPLS models with $Y$ as MDMA response for microdialysate samples collected from saline (left) and MDMA (right) pretreated subjects. Colour-coded grouping based on dose of acute MDMA administration (baseline [BL], 0.0, 5.0, $10.0 \mathrm{mg} / \mathrm{kg}$ ) is shown. 


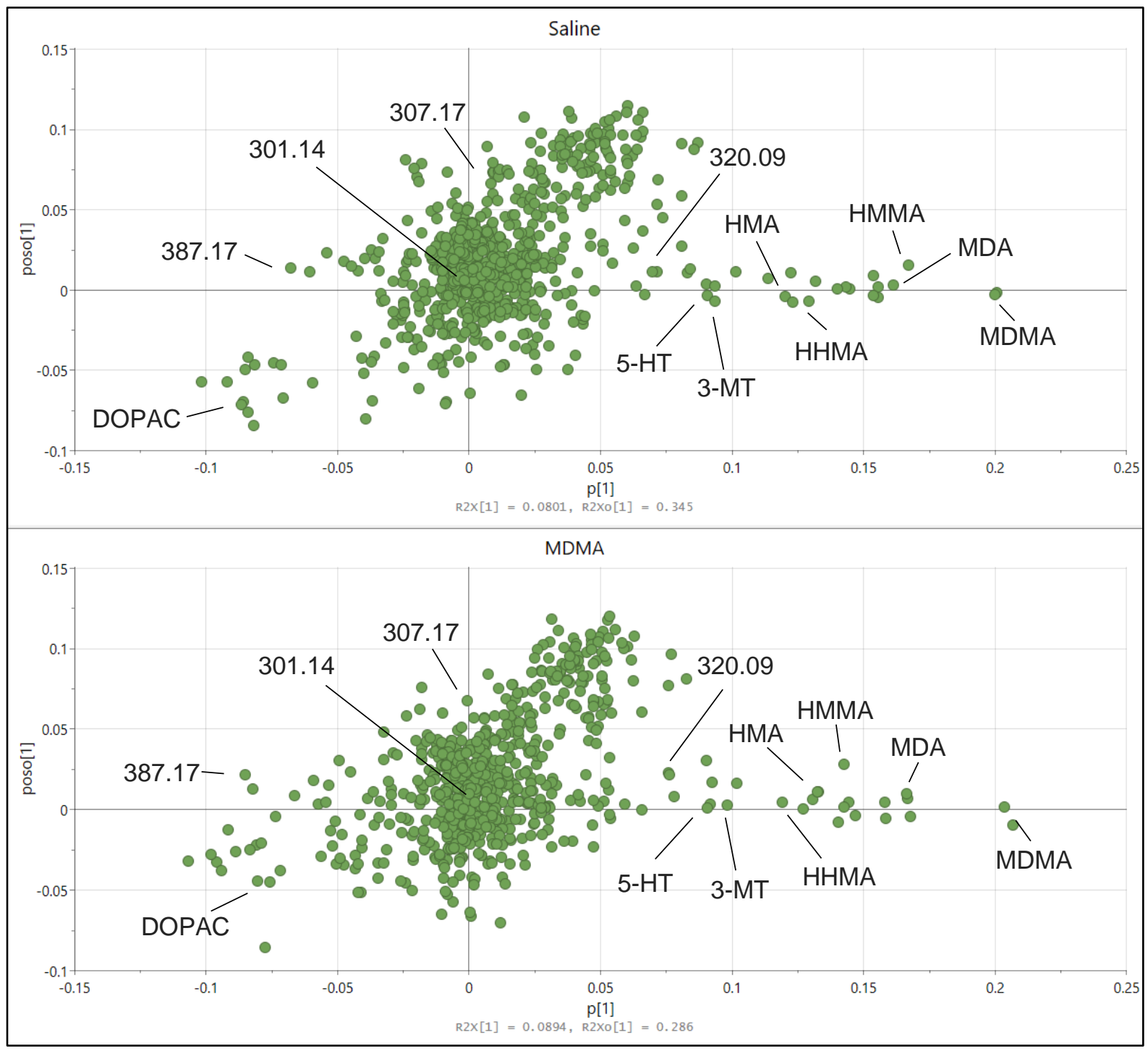

Figure 5.7. Loading plots generated from two OPLS models with $Y$ as MDMA response for microdialysate samples collected from saline (top) and MDMA (bottom) pretreated subjects. Various annotated metabolites of interest and other examples are labelled. 
Identification of metabolites that were relevant to behaviour (criteria 2) was achieved by separate OPLS models for each pretreatment group with the Y variables as locomotor activity, saline: $\mathrm{R}^{2} Y=0.45, \mathrm{Q}^{2}=0.29$; MDMA: $\mathrm{R}^{2} Y=0.61, \mathrm{Q}^{2}=0.48$. The quality of both models was borderline, and less than the previous models using the MDMA response as $Y$, but the results were largely similar. Some small differences were observed, however, such as 3MT having a greater predictive loading for locomotor activity relative to 5-HT. Figure 5.8 shows the score plots for these models with grouping based on the acute dose of MDMA. Figure 5.9 shows the loading plots for these models with similar results to the previous models using MDMA response. 156 variables had a predictive VIP score of $>1$.

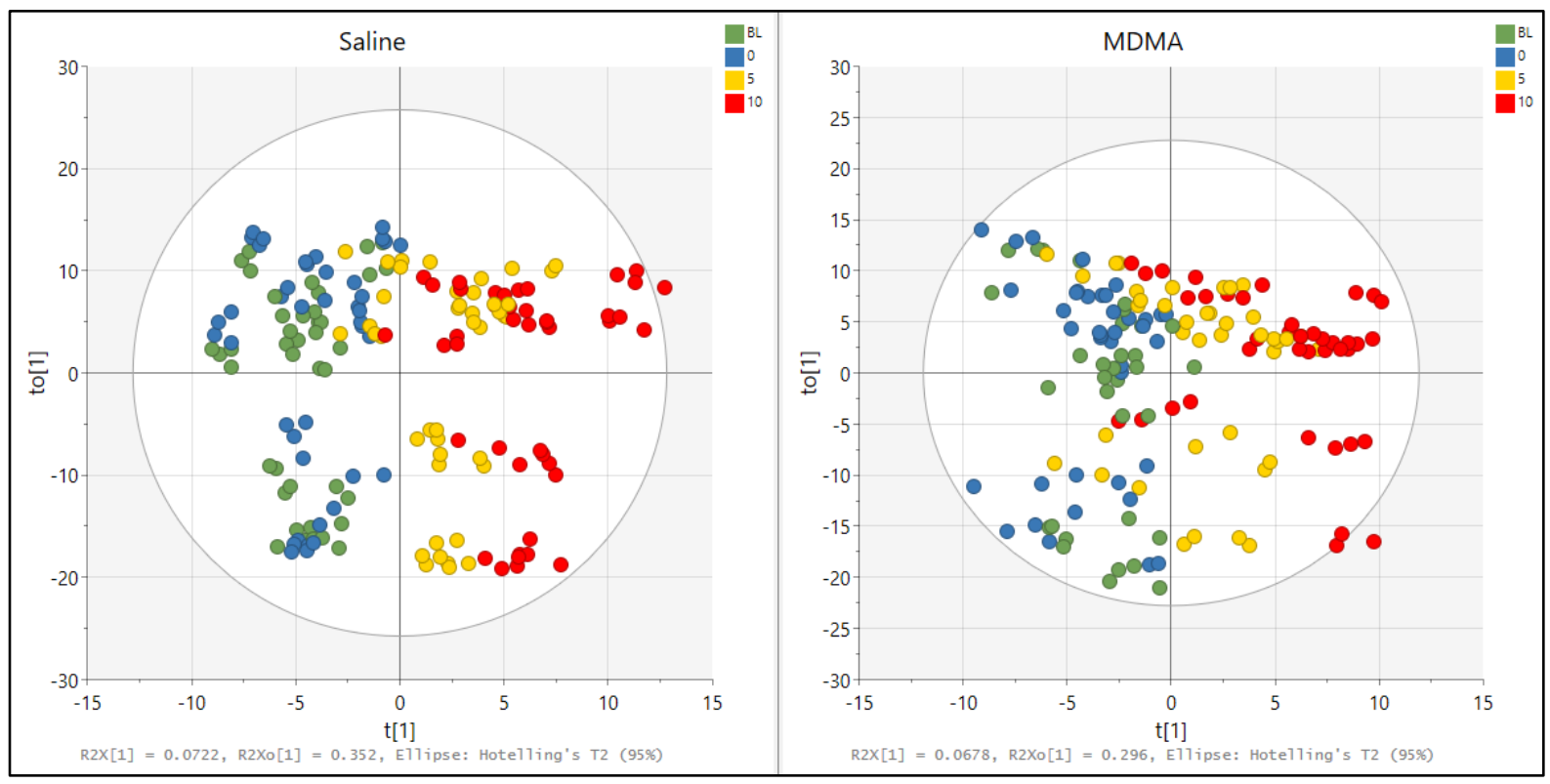

Figure 5.8. Score plots generated from two OPLS models with $Y$ as locomotor activity for microdialysate samples collected from saline (left) and MDMA (right) pretreated subjects. Colour-coded grouping based on dose of acute MDMA administration (baseline [BL], 0.0, 5.0, $10.0 \mathrm{mg} / \mathrm{kg}$ ) is shown. 


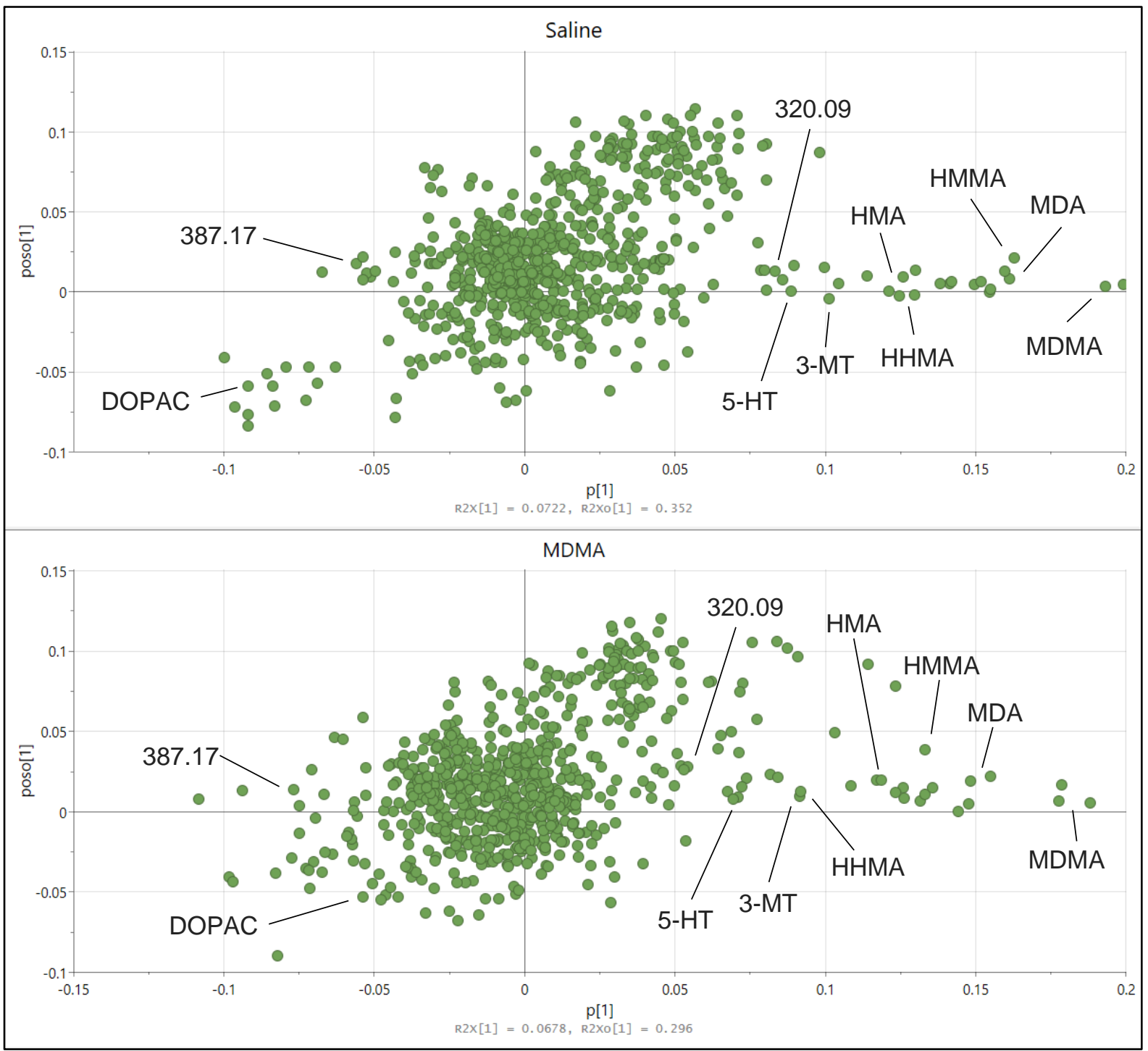

Figure 5.9. Loading plots generated from two OPLS models with $Y$ as locomotor activity for microdialysate samples collected from saline (top) and MDMA (bottom) pretreated subjects. Various annotated metabolites of interest are labelled. 
Identification of metabolites that were relevant to MDMA pretreatment (criteria 3) was assessed in two different ways. First, SUS plots were examined, which compared the predictive variable loadings for the MDMA or locomotor activity response between the saline and MDMA pretreatment groups. Figure 5.10 shows these SUS plots with the predictive correlational loadings (pcorr) for the MDMA group plotted on the x-axis and the saline group plotted on the y-axis. Variables in the upper right quadrant are those that were positively predictive of the MDMA or locomotor activity response in both the saline and MDMA pretreatment groups while those in the lower-left quadrant were negatively predictive in both groups. Variables that deviate from the linear diagonal pattern are those that are predictive of the MDMA/locomotor activity response in one model (i.e. pretreatment group), but not the other. Neither plot showed any such variables, however, suggesting that any group differences were subtle. To further investigate any group differences, three separate OPLS-DA models with dummy $Y$ variables coded as MDMA pretreatment group (saline vs MDMA pretreatment) were generated for baseline/0.0, MDMA 5.0, and MDMA 10.0 samples, baseline/0.0: $\mathrm{R}^{2} Y=$ 0.94, $\mathrm{Q}^{2}=0.71$; MDMA 0.0: $\mathrm{R}^{2} Y=0.99, \mathrm{Q}^{2}=0.86$; MDMA 10.0: $\mathrm{R}^{2} Y=0.92, \mathrm{Q}^{2}=0.68$. Figure 5.11 shows the resulting score plots with a clear separation between the pretreatment groups on the $\mathrm{x}$-axis (predictive component) and large within-group variation on the $\mathrm{y}$-axis (orthogonal component). 218, 195, and 222 variables had a VIP score $>1$ at the baseline/0.0, MDMA 5.0, MDMA 10.0 timepoints/doses, respectively.

Finally, variables that meet all three assessment criteria were identified. The variables that were most relevant to MDMA pretreatment at the baseline/0.0, MDMA 5.0, MDMA 10.0 timepoints/doses (criteria 3) were filtered by the variables that were also most relevant to the MDMA response (criteria 1) and to locomotor activity (criteria 2) based on VIP scores that were $>1$. This left 40, 44, and 61 variables of interest at the baseline/0.0, MDMA 5.0, MDMA 10.0 timepoints/doses that met all three criteria. Many of these variables represented adducts or fragments of the same metabolite, however. Thus, any fragments or adducts that were identified were removed. Some of the variables on this list also had high orthogonal loadings, with highly variable responses between the subjects, and thus, these variables were also removed. A summary of the few remaining unique variables/metabolites/features of interest as well as other notable metabolites discovered throughout the analysis and the assessment criteria that each variable met is shown in Figure 5.12. Follow-up univariate statistics revealed that there were significant increases in relative HMA concentrations at two time points as a function 
of MDMA pretreatment (see Figure 5.12, $p<.05$ ). All other follow-up univariate tests were not significant, $p>0.5$.

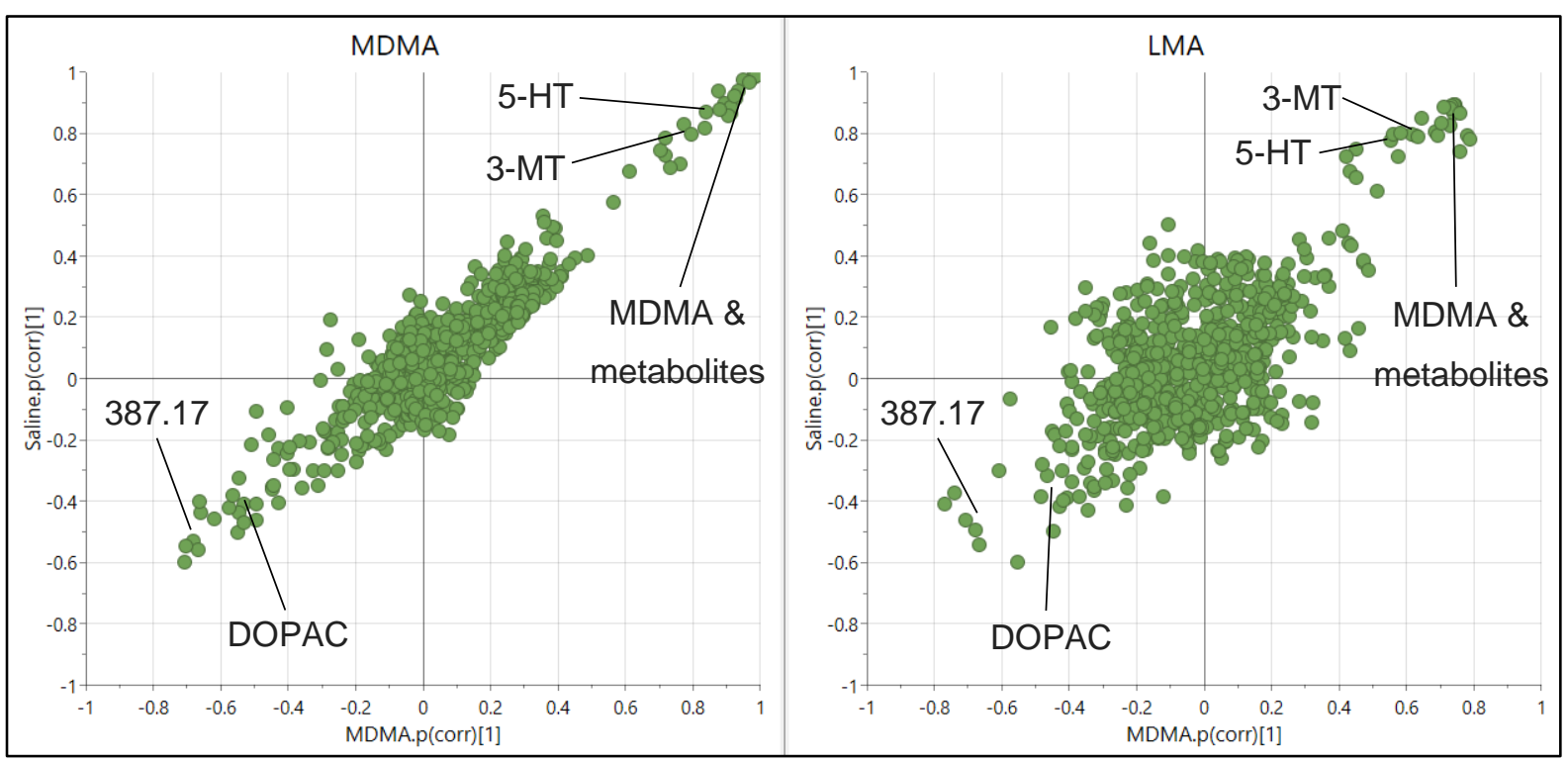

Figure 5.10. Shared and unique structures (SUS) plots comparing predictive correlational loadings (pcorr) for the MDMA response (left) and locomotor activity (LMA; right) between the saline ( $y$-axis) and MDMA pretreated groups ( $x$-axis). Some annotated metabolites of interest are labelled.

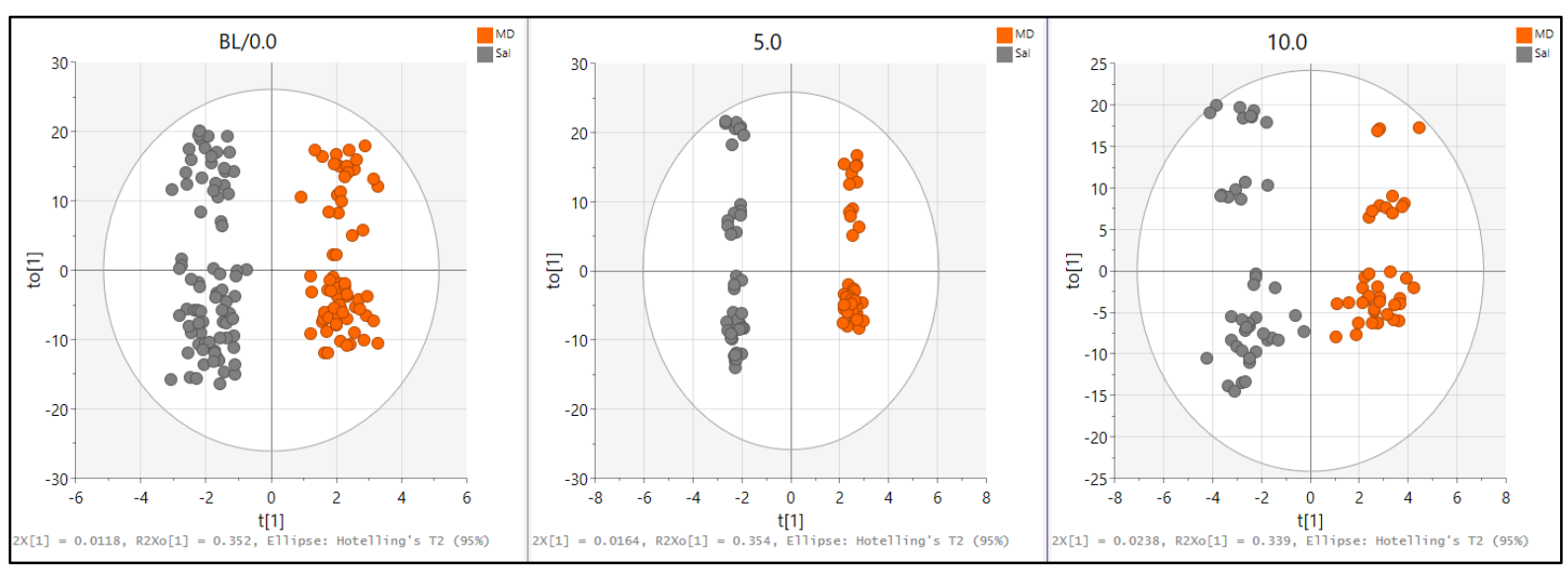

Figure 5.11. Score plots generated from three OPLS-DA models at baseline [BL]/0.0 (left), MDMA 5.0 (centre), and MDMA 10.0 (right) time-points/doses with a dummy $Y$ variable coded as pretreatment group (saline vs MDMA). Colour-coded grouping based on pretreatment group is shown. 
Pre-treatment:

-.. Saline - MDMA
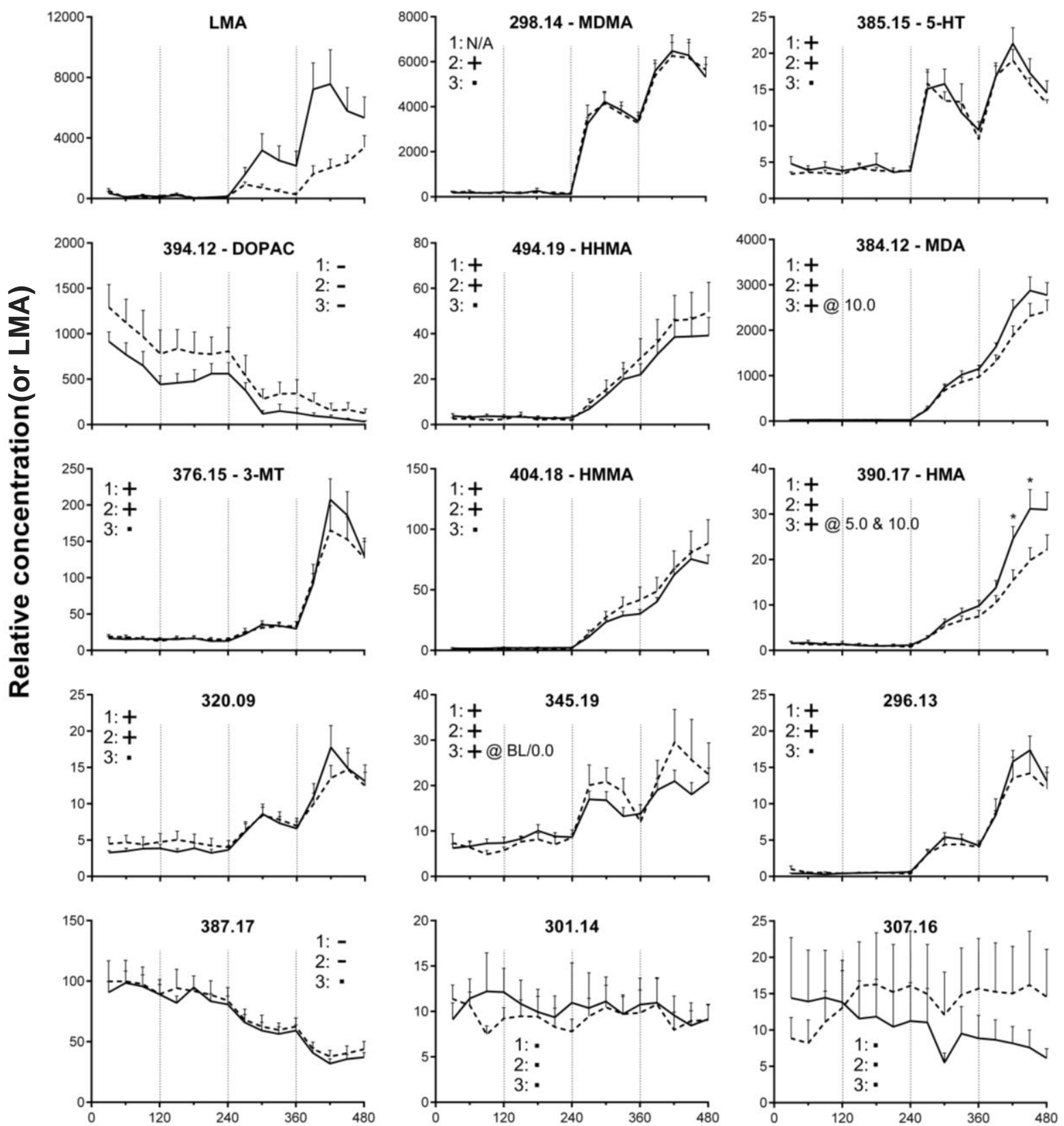

Time (min)

Figure 5.12. Summary of the metabolites of interest and other explanatory examples. Mean relative concentration (or locomotor activity [LMA]; + standard error of the mean) is plotted as a function of time/acute MDMA administration for subjects pretreated with saline (dashed line) and MDMA (solid line). MDMA 0.0, 5.0, and $10.0 \mathrm{mg} / \mathrm{kg}$ i.p. was administered at 120, 240 , and $360 \mathrm{~min}$, respectively. The $\mathrm{m} / \mathrm{z}$ and annotation of each derivatised metabolite is shown at the top of each graph. Legends represent whether the metabolite was positively (+), negatively (-), or neutrally $(\cdot)$ predictive of the MDMA response (criteria 1), LMA (criteria 2), or MDMA pretreatment (criteria 3). $* p<.05$. 


\section{Metabolite annotation}

$298.14 \mathrm{~m} / \mathrm{z}, 385.15 \mathrm{~m} / \mathrm{z}$, and $394.12 \mathrm{~m} / \mathrm{z}$ were known to be benzoylated MDMA, 5-HT, and DOPAC, respectively, from the use of external standards as described in Chapter 4. Targeted MS/MS was used to annotate other selected features of interest. $384.12 \mathrm{~m} / \mathrm{z}$ was confidently annotated as benzoylated MDA. The fragmentations patterns of MDA were very similar to MDMA (Figure 5.13) and to those recorded on the METLIN database. $376.15 \mathrm{~m} / \mathrm{z}$ was annotated as benzoylated 3-MT, giving the same primary fragment of $151.07 \mathrm{~m} / \mathrm{z}$ as that recorded in METLIN as well as $105 \mathrm{~m} / \mathrm{z}$, which is the characteristic fragment of all benzoylated metabolites, including 3-MT (Wong et al., 2016). Less conclusive annotation was given to the other features. $404.18 \mathrm{~m} / \mathrm{z}, 494.19 \mathrm{~m} / \mathrm{z}$, and $390.17 \mathrm{~m} / \mathrm{z}$ were suspected to be benzoylated HMMA, HHMA, and HMA respectively, based on the $\mathrm{m} / \mathrm{z}$ of the multiple detected ions/adducts. Their MS/MS fragmentation patterns are not recorded in METLIN, but HMMA gave a primary fragment of $269.12 \mathrm{~m} / \mathrm{z}$, which would be expected if it fragmented in the same location as MDMA. No prominent fragments other than $105 \mathrm{~m} / \mathrm{z}$ were observed for the other suspected MDMA metabolites, however. The proposed fragmentation of selected annotated metabolites is shown in Figure 5.14. $320.09 \mathrm{~m} / \mathrm{z}$ has the same mass as benzoylated vanillylmandelic acid (VMA; Wong et al., 2016), an end-stage metabolite of catecholamines, but no fragmentation data for this metabolite or any of the other metabolites of interest were obtained. 
Bz MDMA
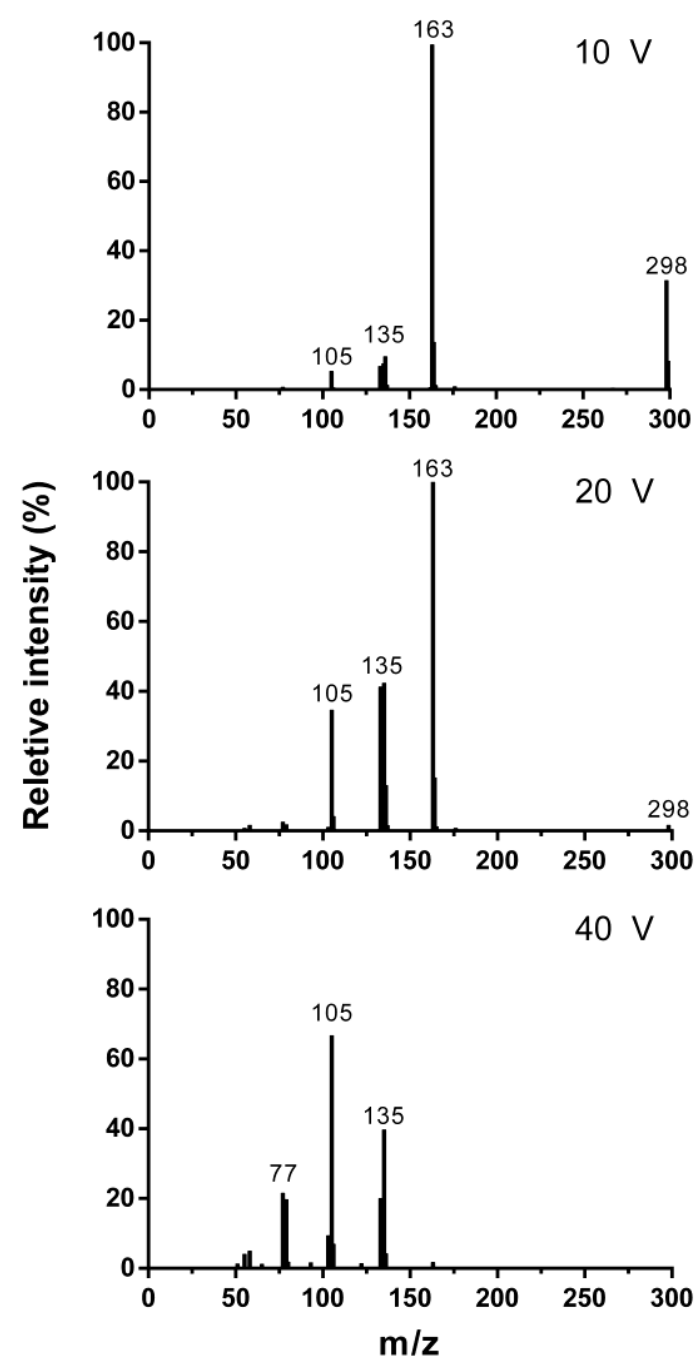

\section{Bz MDA}
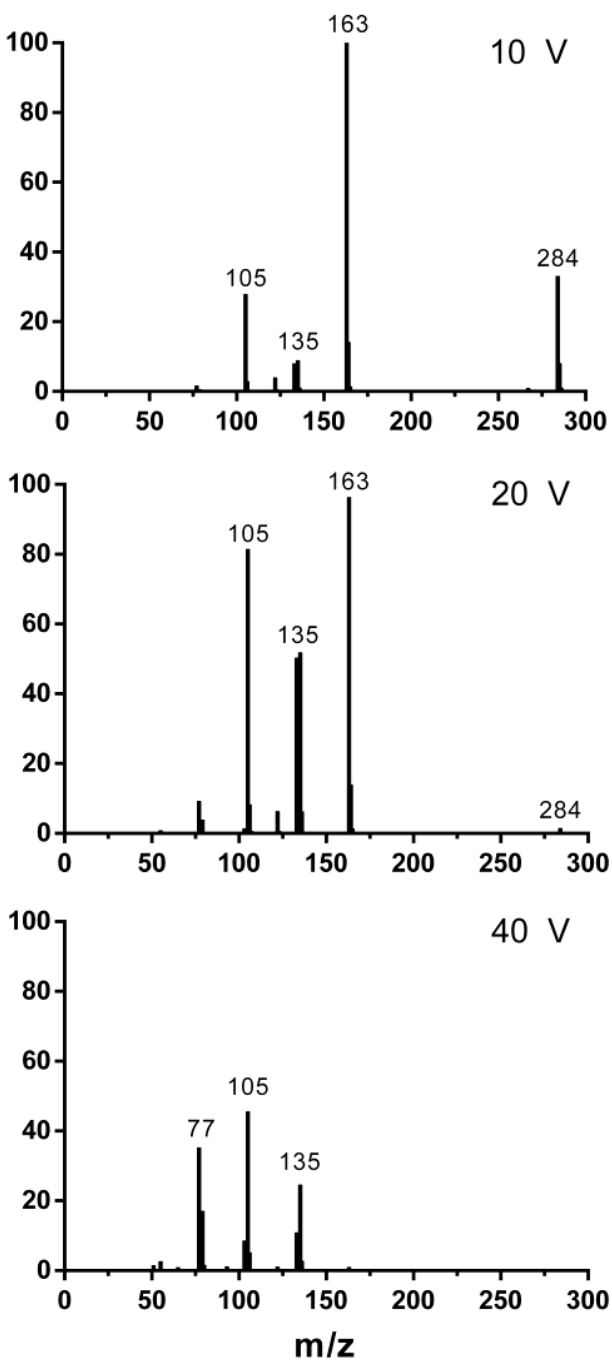

Figure 5.13. Q-TOF ESI MS/MS spectra of benzoylated MDMA (left) and MDA (right) at 10 (top), 20 (middle), and 40 (bottom) eV collision energies. 

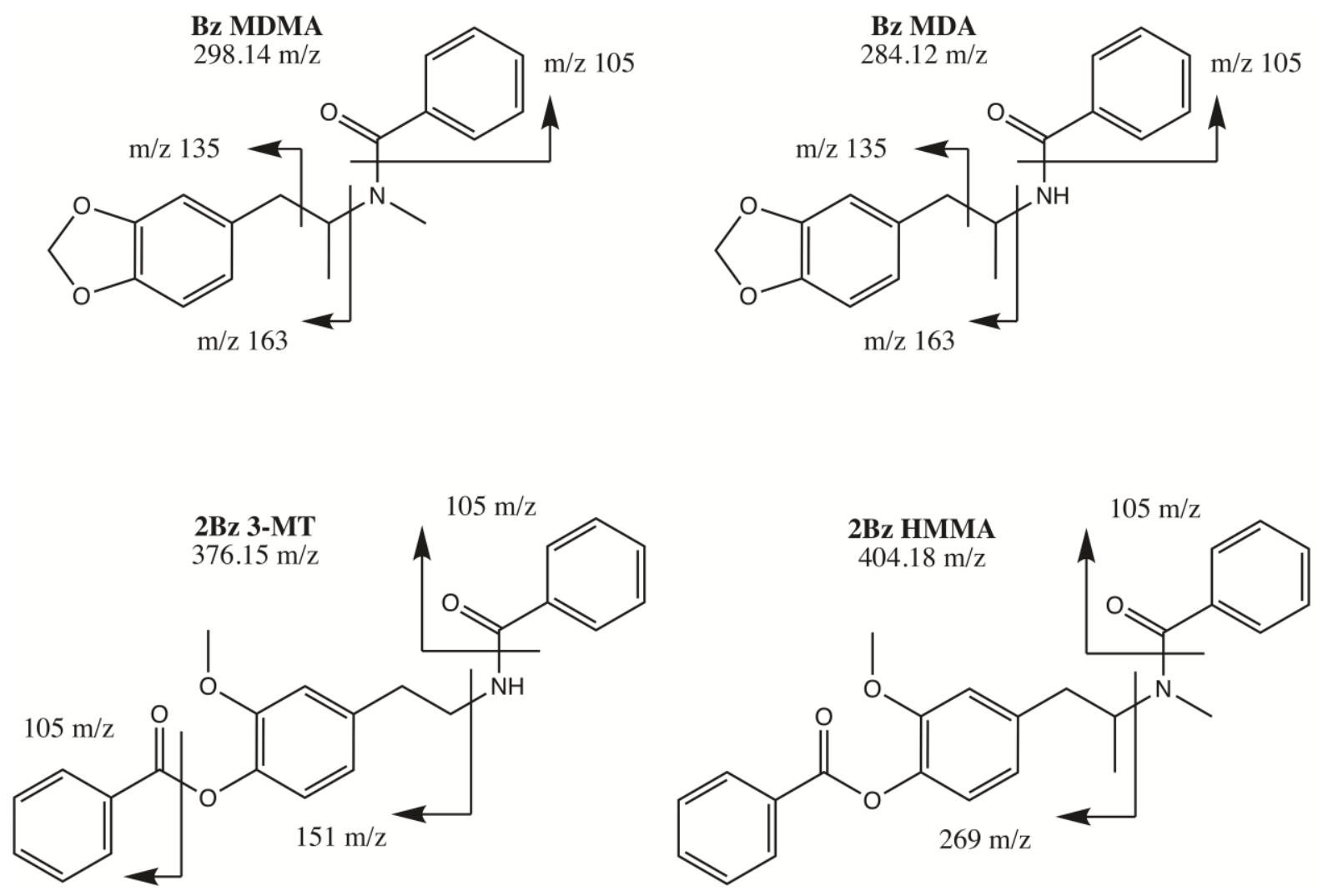

Figure 5.14. Proposed fragmentation of selected metabolites of interest.

\section{Discussion}

In the current study, an untargeted metabolomics procedure was developed to evaluate behaviourally relevant changes in the neuro-metabolomic profile of rats as a function of repeated MDMA exposure. Microdialysis samples were collected from the medial striatum at 30 min intervals following the administration of ascending doses of MDMA from subjects pretreated with either saline or a behaviourally sensitising regimen of repeated MDMA exposure. During this time, locomotor activity was also measured. Metabolites of interest were assessed by PCA, OPLS, and OLPS-DA based on three criteria. 1) The metabolite was relevant to acute MDMA administration, and its concentration changed dose-dependently over time. 2) The metabolite was relevant to MDMA-produced behaviour, and its concentration was predictive of locomotor activity. 3) The metabolite was relevant to repeated MDMA exposure, and its concentrations at baseline or following acute MDMA administration changed as a function of MDMA pretreatment. While numerous metabolites met some of these criteria, very few met all three. 
Several metabolites were found to be relevant to acute MDMA administration, and their concentration changed dose-dependently over time (criteria 1). Unsurprisingly, the metabolites that were most predictive of the measured MDMA response were the adducts and fragments of MDMA, MDA, and what was suspected to be HMMA, HHMA, and HMA. Other metabolites that were dose-dependently impacted by acute MDMA administration included 5HT, DOPAC, and 3-MT, as has been previously demonstrated in mice or rats (Baumann, Clark, Franken, et al., 2008; Baumann, Clark, \& Rothman, 2008; Górska \& Gołembiowska, 2015; Gough et al., 2006; Kankaanpää et al., 1998; Kurling et al., 2008; Nair \& Gudelsky, 2004; O’Shea et al., 2005; Shankaran \& Gudelsky, 1999). Several unknown metabolites were also dose-dependently impacted by acute MDMA administration including 320.09 m/z, $345.19 \mathrm{~m} / \mathrm{z}$, $296.13 \mathrm{~m} / \mathrm{z}$, and $387.17 \mathrm{~m} / \mathrm{z}$. One of these unknown metabolites $(320.09 \mathrm{~m} / \mathrm{z})$ may have been VMA, an end-stage metabolite of catecholamines. Another one of these metabolites (387.17 $\mathrm{m} / \mathrm{z}$ ) decreased in a remarkedly dose-dependent manner with acute MDMA administration. This, however, might be an artefact of ion suppression since this metabolite co-eluted with the highly abundant MDMA at 4 min.

Several metabolites were also determined to be predictive of MDMA-produced locomotor activity (criteria 2). Unsurprisingly, these metabolites were similar to those that met criteria 1 since locomotor activity also increases dose-dependently with MDMA administration. MDMA and its metabolites were most strongly predictive of locomotor activity, followed by 3-MT and 5-HT. MDMA has long been known to produce dosedependent increases in locomotor activity, as was demonstrated in Chapter 4. 5-HT has also been previously implicated in the acute locomotor activating effects of MDMA (Berger et al., 1992; Callaway, Rempel, Peng, \& Geyer, 1992; Callaway, Wing, \& Geyer, 1990; Hekmatpanah \& Peroutka, 1990; Kehne et al., 1996), while 3-MT is the primary metabolite of DA, which is well known to be an important driver of MDMA and other drug-produced locomotor activity (Ball, Budreau, \& Rebec, 2003; Bubar, Pack, Frankel, \& Cunningham, 2004; Daniela et al., 2004; L. H. Gold, Hubner, \& Koob, 1989; Kehne et al., 1996). It should be noted that in pilot metabolomics analyses of samples collected from smaller populations of rats throughout the method development described in Chapter 4 (when DA was able to be reliably measured), DA was consistently one of the metabolites most predictive of MDMAproduced locomotor activity (data not shown).

The concentration of numerous metabolites was also predictive of repeated MDMA exposure. The relevance of many of these metabolites to the development and expression of 
behavioural sensitisation was questionable, however. Metabolites that were not also impacted by acute MDMA administration and predictive of MDMA-produced locomotor activity were unlikely to be relevant. Thus, these metabolites were excluded, and only those that met all three assessment criteria were examined further.

The final results suggest potential changes in MDMA metabolism. Although the concentration of MDMA itself did not change as a function of repeated MDMA exposure, the concentration of some of the metabolites of MDMA did change with repeated exposure and some met all three assessment criteria (see Figure 1.1 for review on MDMA metabolism). All detected metabolites, MDA, HMA, HHMA, and HMMA, increased dose-dependently with acute MDMA administration (criteria 1), as would be expected, and were positively predictive of locomotor activity (criteria 2). Concentrations of MDA, one of two primary metabolites of MDMA, were higher following $10 \mathrm{mg} / \mathrm{kg}$ MDMA in pretreated rats, while concentrations of HMA, a metabolite of MDA, were also higher following 5 and $10 \mathrm{mg} / \mathrm{kg}$ MDMA in pretreated rats (criteria 3). These results were mirrored, although non-significantly, by marginal decreases in HHMA, the second primary metabolite of MDMA, and HMMA, a metabolite of HHMA, in MDMA pretreated rats. These results might suggest an increase in one MDMA metabolism pathway relative to the other as a function of repeated MDMA exposure. MDA is a particularly potent psychostimulant in itself and has been shown to be more effective at stimulating locomotor activity than equivalent doses of MDMA (20 mg/kg i.p.; Bexis \& Docherty, 2006). Thus, increased extracellular concentrations of MDA might contribute to the sensitised behavioural response observed in the current study. HMA and the other metabolites of MDMA have been previously shown to have a minimal direct effect on locomotor activity, however (Schindler, Baumann, Thorndike, Blough, \& Goldberg, 2012; Yeh \& Hsu, 1991). The pharmacokinetics of MDMA and its metabolites (MDA, HHMA, HMMA) in rat serum have been previously examined prior to, and following, the self-administration of a moderate amount of MDMA (Bradbury et al., 2013). Although there were no significant differences in any of the measures, the authors did note a tendency for greater values in rats that acquired MDMA selfadministration on some measures. Further research with a targeted approach would be needed to elucidate the current findings and determine the role of changes in MDMA metabolism in behavioural sensitisation.

The final results also suggest potential changes in DA metabolism. Concentrations of 3-MT increased dose-dependently with acute MDMA administration, were positively predictive of locomotor activity, and were marginally higher following the administration of 
$10 \mathrm{mg} / \mathrm{kg}$ MDMA in MDMA pretreated rats. This might suggest a sensitised DAergic response following MDMA pretreatment, as was suggested in Chapter 4, and as has been previously demonstrated following sensitising regimens of repeated MDMA exposure (Kalivas et al., 1998; Morgan et al., 1997). In contrast, concentrations of DOPAC decreased dose-dependently with acute MDMA administration, were negatively predictive of locomotor activity, and were decreased at baseline and following MDMA administration in MDMA pretreated rats. It should be noted that the sample preparation procedures used in the current study were not ideal for measuring DOPAC, however, as was described in Chapter 4, and large variation was observed in DOPAC measurements. Nevertheless, these results are consistent with what was found throughout the method development phase and with previous studies (Gough et al., 2006; Nash, 1990).

Another metabolite $(345.19 \mathrm{~m} / \mathrm{z})$ also met all three criteria. The concentration of this metabolite increased dose-dependently with acute MDMA administration, was positively predictive of locomotor activity, and was decreased during baseline/0.0 conditions in MDMA pretreated rats. This metabolite was not identified or annotated, however. Of course, it needs to be stated that most of the final results discussed here were not significant with follow-up univariate statistics, and thus, these findings should be interpreted with caution. Nonetheless, the current research serves as a proof of concept and demonstrates the potential power of a microdialysis and LC-MS-based time-series metabolomics procedure that incorporates behavioural measures. 


\section{CHAPTER 6: GENERAL DISCUSSION}

Parts of this chapter have been adapted with licensed permission from work published in Addiction Biology (van de Wetering \& Schenk, 2019).

MDMA/ecstasy is a popular recreational drug of abuse. Like other drugs of abuse, a proportion of MDMA/ecstasy users develop symptoms characteristic of a SUD. Despite this, the behavioural and neurobiological effects of repeated MDMA exposure are relatively less well understood than other drugs. The purpose of the current research was to investigate behaviourally relevant neuroadaptations that develop with repeated MDMA exposure in rats.

\section{Summary of findings}

In Chapter 2, the accumulation of the transcription factor, $\Delta \mathrm{FosB}$, following chronic self-administered MDMA, was mapped across numerous brain regions using IHC. MDMA self-administration produced an accumulation of $\Delta \mathrm{FosB}$ in several of the same brain regions as repeated exposure to other drugs of abuse. These brain regions are known to play crucial roles in the development and maintenance of drug addiction. Striatal regions that are involved in goal-directed behaviour (NAcc, DMS) showed a particularly pronounced accumulation of $\Delta$ FosB. Brain regions involved in several other important aspects of addiction, such as executive functioning and conditioning also showed increased $\Delta$ FosB expression. These regions included the CeA, BLA, and most major subdivisions of the PFC. It has been suggested that MDMA self-administration progresses as a function of neuroadaptive responses in the same brain regions that mediate the progression of self-administration of other drugs of abuse (Schenk, 2011). The present data are consistent with this idea and also suggest that $\Delta$ FosB accumulation in several brain regions that are relevant to addiction is a common consequence of repeated exposure to drugs of abuse.

One of the key findings from Chapter 2, was the region-specific increases in striatal $\Delta \mathrm{FosB}$; significant increases in $\Delta \mathrm{FosB}$ levels were observed within the ventral and dorsomedial regions, but not the dorsolateral regions. In Chapter 3, the idea that these region-specific increases in striatal $\triangle \mathrm{F}$ osB were relevant to the sensitisation of MDMA-produced behavioural responses following repeated exposure was determined. Following repeated, systemic MDMA exposure, local administration of MDMA into the ventral or dorsomedial striatum, but not the dorsolateral striatum resulted in the expression of behavioural sensitisation. These results mirrored those from Chapter 2. Together, these findings suggested that MDMA-produced 
increases in $\Delta \mathrm{FosB}$ within these brain regions might be involved in the development and/or expression of sensitised responses to MDMA following repeated exposure, as has been shown with other drugs of abuse (Brenhouse \& Stellar, 2006; Colby et al., 2003; Conversi et al., 2007; Grueter et al., 2013; Kaplan et al., 2011; Kelz et al., 1999; Marttila et al., 2006; McDaid et al., 2006; Zachariou et al., 2006).

In Chapter 4, a targeted investigation of the more specific neurochemical correlates of sensitised MDMA-produced behaviour was carried out. A procedure for the quantification of extracellular medial-striatal concentrations of 5-HT, DA, and MDMA was developed that utilised microdialysis and LC-MS. Repeated MDMA exposure had no effect on extracellular concentrations of MDMA following acute systemic administration, suggesting that behavioural sensitisation and any resulting neurochemical changes were not due to differences in MDMA uptake. Baseline or MDMA-produced 5-HT was not significantly impacted by repeated MDMA exposure and did not correlate well with sensitised locomotor activity suggesting that MDMA-produced 5-HT release in the medial striatum is not an important mechanism by which repeated MDMA exposure results in behavioural sensitisation. In contrast, extracellular concentrations of DA at baseline and following MDMA administration were enhanced following repeated exposure and correlated well with sensitised locomotor activity. Unfortunately, these DA results were incomplete and were only collected from a small number of subjects. Nonetheless, these tentative findings are consistent with those found previously using a similar dosing regimen of repeated MDMA exposure (Kalivas et al., 1998) and with the general documented role of DAergic mechanisms in behavioural sensitisation to other drugs of abuse (for reviews see Pierce \& Kalivas, 1997; T. E. Robinson \& Berridge, 1993; Vanderschuren \& Pierce, 2010; Vezina, 2004).

In Chapter 5, untargeted metabolomics was used in an attempt to identify novel or unexpected compounds that are relevant to MDMA behavioural sensitisation. A procedure for the analysis of behavioural data in combination with time-series metabolomics data collected via microdialysis was developed. The goal was to identify metabolites that 1) were dosedependently impacted by acute MDMA administration, 2) were relevant to MDMA-produced behaviour, and 3) were impacted by repeated MDMA exposure. The results primarily served as a proof of concept, highlighting the potential power of a microdialysis and LC-MS-based time-series metabolomics procedure that incorporates behavioural measures. Nonetheless, some potentially interesting results were obtained. Potential changes in one pathway of MDMA metabolism relative to the other were observed. Potential changes in DA metabolism were also 
observed, which would be consistent with the tentative findings of Chapter 4 and with previous literature (Colussi-Mas et al., 2010; Kalivas et al., 1998; Morgan et al., 1997; Schenk, 2011; van de Wetering \& Schenk, 2017). Some potentially interesting unknown metabolites were also identified.

\section{Relevance and value of findings}

\section{Self-administration and $\Delta$ FosB}

Repeated exposure to drugs of abuse produces a plethora of neuroadaptations that, in ways not yet fully understood, foster continued and escalating drug-seeking/taking behaviour. Identification of these neuroadaptations and a comprehensive understanding of how they underlie such addictive behaviour is a crucial step towards the development of therapeutic interventions that can prevent or reverse these maladaptive neuroadaptations and restore normal behaviour. Like most other research with this overarching goal, the current research employed animal models to investigate the neuroadaptations that develop with repeated MDMA exposure.

Long-access self-administration sessions and an escalation of drug-intake have been suggested to be of particular importance for the comprehensive and valid modelling of a SUD since these conditions have been shown to exacerbate, or are often necessary for, the development of several features that are characteristic of a SUD (Ahmed, 2011; Ahmed \& Koob, 1998; Ahmed et al., 2000; Edwards \& Koob, 2013; Kitamura et al., 2006; O’Dell et al., 2007; A. J. Roberts et al., 2000). It is for these reasons that the first experiment of the current research employed a minimum of 29 long-access (6 hour) MDMA self-administration sessions to identify brain regions that have undergone significant neuroadaptive change, as measured by $\Delta$ FosB accumulation. These self-administration conditions resulted in a significant escalation of MDMA-intake and have been previously shown to produce extensive drug- and cue-produced MDMA-seeking (Schenk et al., 2016, 2011).

The current research demonstrated, for the first time, the impact of repeated MDMA exposure on the accumulation of $\Delta \mathrm{FosB}$ in a number of brain regions that have been implicated in addiction. $\triangle \mathrm{FosB}$ has been suggested to be particularly relevant to addiction for a number of reasons (for reviews see McClung et al., 2004; Mews et al., 2019; Nestler, 2008; Nestler et al., 1999; Robison \& Nestler, 2011). To summarise, the protein is induced in the brain by repeated exposure to virtually all drugs of abuse (now also including MDMA). Because $\Delta$ FosB is highly stable, it can persist in the brain for many weeks or even months following stimulus exposure, rather than a few hours like most transcription factors. This allows the $\Delta \mathrm{FosB}$ to accumulate at 
very high levels following repeated drug exposure and have a long-lasting impact on gene transcription. Further, $\Delta$ FosB has been shown to be both sufficient and necessary for the development of multiple behavioural phenotypes that are relevant to addiction, such as sensitisation. Lastly, $\Delta$ FosB has also been identified as an important, and in some cases, a crucial mediator of many relevant neuroadaptations produced by repeated exposure to drugs of abuse.

There is evidence to suggest that this last point may also hold true for MDMA. For example, repeated MDMA exposure increased dendritic spine density and spine count on MSN in the NAcc and increased spine density on distal dendrites of output neurons in the mPFC; an effect that was accompanied by sensitised behavioural responses to MDMA (Ball et al., 2009). These effects might be mediated by $\Delta$ FosB since $\Delta$ FosB has been shown to mediate the transcription of numerous genes involved in dendritic spine formation and structural plasticity (Bibb et al., 2001; Maze et al., 2010; Nestler, 2008, 2012; Robison \& Nestler, 2011; Winstanley et al., 2009) and increased $\Delta$ FosB was observed within these brain regions following repeated exposure in the current research.

Other neuroadaptive responses produced by repeated MDMA exposure may also be mediated by $\triangle$ FosB. Repeated, intermittent, MDMA exposure upregulated the expression of GluR2, which codes for an AMPA receptor subunit, in the cortex and caudate as well as CaMKII, which phosphorylates various glutamate receptors, in the hippocampus (KindlundhHögberg, Blomqvist, Malki, \& Schiöth, 2008; Moyano, Del Río, \& Frechilla, 2005; Moyano, Frechilla, \& Del Río, 2004). Glutamate receptors have been implicated in the rewarding effects of MDMA (García-Pardo, Miñarro, \& Aguilar, 2018; García-Pardo, Miñarro, Llansola, Felipo, \& Aguilar, 2019) as well as other psychostimulant drugs (Kauer \& Malenka, 2007; Kelz et al., 1999; T. E. Robinson \& Kolb, 2004) and the expression of both of these proteins has been shown to be positively mediated by $\Delta$ FosB (Bibb et al., 2001; Kelz et al., 1999; McClung \& Nestler, 2003; Peakman et al., 2003). Repeated MDMA exposure also produced changes in the expression of the opioid peptide, dynorphin, in various brain regions including the striatum and VTA (Di Benedetto et al., 2011; Di Benedetto, D’Addario, Candeletti, \& Romualdi, 2006). Dynorphin regulates DAergic output from the VTA and the gene that encodes dynorphin is similarly mediated by $\Delta$ FosB (Bruchas et al., 2010; Zachariou et al., 2006). These findings, together with those from the current research, suggest that $\Delta \mathrm{FosB}$ might mediate several neuroadaptive responses to repeated MDMA exposure that are relevant to sensitisation and reinforcement. 


\section{Behavioural sensitisation and the acquisition of self-administration}

It has long been known that repeated exposure to some drugs of abuse can result in the persistent sensitisation of their behavioural effects such as locomotor activity (Downs \& Eddy, 1932; Seevers \& Tatum, 1931). Evidence implicating the relevance of this phenomenon to addiction has accumulated over the years and can be summarised by three main points (for reviews see Berridge \& Robinson, 2016; Pierce \& Kalivas, 1997; T. E. Robinson \& Berridge, 1993, 2008; Vanderschuren \& Pierce, 2010; Vezina, 2004). First, repeated drug exposure under conditions that result in behavioural sensitisation can also sensitise the reinforcing effects of drugs, as evidenced by the facilitated acquisition of self-administration, higher breakpoints on PR schedules, and increased CPP. Second, manipulations that prevent the development of behavioural sensitisation also prevent the sensitisation of the reinforcing effects of drugs. Third, the neural mechanisms underlying the development and expression of these sensitised responses appear to overlap considerably and involve various neuroadaptations in brain regions such as the PFC, VTA, NAcc, and dorsal striatum.

The subsequent experiments of the current research utilised a behavioural sensitisation procedure to further investigate some of the brain regions that differentially expressed $\Delta$ FosB following MDMA self-administration. The pretreatment regimen of repeated MDMA exposure used to induce behavioural sensitisation in the current research has been previously shown to also sensitise the reinforcing effects of MDMA, as indicated by the facilitated acquisition of self-administration (van de Wetering \& Schenk, 2017). It would, therefore, be expected that the results of the current research are also relevant to the acquisition of MDMA selfadministration.

The current research demonstrated that local injections of MDMA into the dorsomedial and ventral striatum, but not the dorsolateral striatum, resulted in the expression of behavioural sensitisation following repeated systemic exposure. These results would suggest that the ventral and dorsomedial striatum are also important for the sensitised acquisition of MDMAself-administration. This might be expected given the documented role of these brain regions in goal-directed behaviour (Corbit et al., 2012; Ito et al., 2002; Murray et al., 2012; Vanderschuren et al., 2005; Zapata et al., 2010). As previously mentioned, $\Delta$ FosB expression within these brain regions may also be important and might mediate neuroadaptive responses such as changes in dendritic spine density that are associated with the development of these sensitised responses (Ball et al., 2009). This has been previously demonstrated with other drugs of abuse. An increase in both the locomotor activating and reinforcing effects of cocaine was 
observed following striatal or accumbal overexpression of $\Delta$ FosB (Colby et al., 2003; Kelz et al., 1999), and this was accompanied by an increase in the number and density of dendritic spines in $\mathrm{D}_{1}$-type MSN (Grueter et al., 2013).

The current research also provided some insight on the neurotransmitter systems that are involved in the development and expression of sensitised responses to MDMA. Baseline or MDMA-produced medial-striatal 5-HT concentrations were not impacted by repeated MDMA exposure, nor did they correlate well with sensitised locomotor activity. These results would suggest that 5-HT is similarly not important for the sensitised acquisition of MDMA self-administration. The role of 5-HT in the development and expression of sensitised responses to MDMA has not been previously examined. However, there is evidence for an inhibitory role of 5-HT in the normal acquisition of MDMA self-administration. Rats with a greater NAcc 5-HT response to MDMA (3 mg/kg, i.v.) were less likely to subsequent meet acquisition criteria for MDMA self-administration (Bradbury et al., 2013) while 5,7-DHT lesions (Bradbury et al., 2013) or a genetic mutation of the 5-HT transporter (Oakly et al., 2013) greatly facilitated the acquisition of MDMA self-administration. Given that repeated MDMA exposure can produce various 5-HTergic deficits, deficits that would be expected to facilitate the acquisition of MDMA sensitisation, the results of the current research were somewhat surprising. One explanation is that the challenge doses used in the current study, although ideal for assessing behavioural sensitisation, were not ideal for assessing changes in MDMAproduced 5-HT or the reinforcing effects of self-administered MDMA. As previously discussed, these doses used were near the top end of the dose-effect curve for MDMA-produced 5-HT release and transporter binding (Battaglia, Brooks, et al., 1988; Green et al., 2003; Rudnick \& Wall, 1992; Schmidt et al., 1987). These doses are also much higher than what is typically received during self-administration $(0.5$ or $1.0 \mathrm{mg} / \mathrm{kg} / \mathrm{infusion})$. Thus, a lower dose may have been more appropriate for revealing any potential 5-HT deficits that are relevant to changes in the reinforcing efficacy of self-administered MDMA. Another, more parsimonious explanation is that the regimen of exposure in the current study simply had no significant effect on extracellular medial-striatal 5-HT and other mechanisms underlie the previously observed sensitised acquisition of MDMA self-administration (van de Wetering \& Schenk, 2017).

One such likely mechanism is the sensitisation of central DAergic mechanisms, which have been demonstrated to underlie the sensitisation of the reinforcing effects of other drugs of abuse following repeated exposure (Cador et al., 1995; Kalivas \& Weber, 1988; Pierre \& Vezina, 1998; Vezina, 1993, 1996; Vezina et al., 2002; Vezina \& Stewart, 1989). Like other 
drugs of abuse, DAergic mechanisms play an important role in the acquisition of MDMA selfadministration. For example, rats that met acquisition criteria for MDMA self-administration displayed greater MDMA-produced extracellular striatal DA compared to rats that failed to acquire self-administration (Colussi-Mas et al., 2010). This suggests that the development of a sensitised DAergic response to MDMA might contribute to the acquisition of MDMA selfadministration. In support of this idea, previous studies have shown that MDMA-produced DA release (Kalivas et al., 1998) and tissue levels of DA (Mayerhofer et al., 2001) were increased following repeated exposure. Cross-sensitisation to both the reinforcing (Fletcher, Robinson, \& Slippoy, 2001) and DA-releasing (Kalivas et al., 1998; Morgan et al., 1997) effects of cocaine has also been observed following repeated MDMA exposure. In the current research, DA concentrations at baseline as well as following MDMA administration appeared to be enhanced following repeated exposure and correlated well with sensitised locomotor activity. Additional changes in multiple DA metabolites were also apparent. These tentative results would suggest that the previously observed sensitised acquisition of MDMA selfadministration (van de Wetering \& Schenk, 2017) might be similarly due to sensitised DAergic mechanisms, as has been shown with other drugs of abuse (Berridge \& Robinson, 2016; Vanderschuren \& Pierce, 2010; Vezina, 2004).

\section{Metabolomics}

Only a handful of previous studies have used untargeted metabolomics to examine the effect of repeated exposure to drugs of abuse (see Table 5.2 for a summary). In one study, the effect of withdrawal from repeated morphine treatment was examined using nuclear magnetic resonance (NMR; Deng et al., 2012). Several changes in metabolites related to energy or neurotransmission (glutamate/GABA) were observed, and compellingly, some of these changes were reversed following methadone or clonidine intervention. The same research group also found several changes in metabolites related to energy or neurotransmission (glutamate/GABA/acetylcholine/tryptamine) following a regimen of nicotine exposure that resulted in CPP (H. Li et al., 2014). Another lab group used gas-chromatography MS (GC-MS) to examine the effect of repeated heroin (Zheng et al., 2013) or methamphetamine (Zheng et al., 2014) exposure. In these studies, multiple samples were collected during and after drug treatment in order to provide some temporal information on any metabolomic changes. Following repeated heroin exposure, changes in the concentration of metabolites associated with the tricarboxylic acid cycle were found as well as changes in 5-HT and tryptophan, which had restored to baseline following 4 days of withdrawal (Zheng et al., 2013). Following 
repeated methamphetamine exposure, multiple changes in metabolites related to energy metabolism and neurotransmission (glutamate/aspartate) were also found, most of which had similarly restored to baseline following 2 days of withdrawal (Zheng et al., 2014). Another study examined the effect of chronic alcohol vapour exposure on the metabolome using direct injection MS (Meinhardt et al., 2015). Notable results included changes in levels of DA and Met-enkephalin. In a recent study, the effect of self-administered heroin on the metabolomic profile of serum samples collected after a drug-induced reinstatement session was examined (Ning, Leng, Chen, Ma, \& Gong, 2018). Several metabolites, including those related to the tricarboxylic acid cycle, keto bodies, and neurotransmitter precursors (choline, glutamine, and phenylalanine), were impacted compared to drug-naive controls. In another study, the effect of repeated morphine, methamphetamine, or cocaine exposure, under conditions that produced CPP, was examined (Zaitsu et al., 2014). Consistent with other studies, they found changes in the concentrations of metabolites related to the tricarboxylic acid cycle following morphine treatment. The concentration of several other metabolites (e.g. 3-hydroxybutyric acid, Ltryptophan, cystine) was also impacted depending on the drug.

Table 5.2.

Summary of metabolomics studies

\begin{tabular}{lllll}
\hline Citation & Subjects & Drug & Sampling & Analysis \\
\hline Deng et al. (2012) & Rhesus & Morphine & Tissue from & NMR \\
& monkey & & $\begin{array}{l}\text { hippocampus and } \\
\text { PFC }\end{array}$ & \\
& & & Tissue from & NMR \\
Li et al. (2014) & Mouse & Nicotine & hippocampus, PFC, & \\
& & & and striatum & \\
Zheng et al. (2013) & Rat & Heroin & Serum and urine & GC-MS \\
Zheng et al. (2014) & Rat & Methamphetamine & Serum and urine & GC-MS \\
Meinhardt et al. (2015) & Rat & Ethanol & Tissue from PFC & MS \\
& & & and striatum & \\
Ning et al. (2018) & Rat & Heroin & Serum & NMR \\
Zaitsu et al. (2014) & Rat & Morphine & Serum and urine & GC-MS \\
& & Methamphetamine & & \\
& & Cocaine & &
\end{tabular}


These studies illustrate the advantages of the procedure developed in the current research. In all of these studies, a complete lack or very poor temporal information was obtained due to the sampling procedures used (e.g. brain tissue or anesthetised serum collection). When some temporal information was provided, multiple class-based comparisons (i.e. PLS-DA or OPLS-DA) were carried out at each time-point, and the temporal information was rarely incorporated into the analyses. Further, few studies took behavioural measures, and for those that did (e.g. CPP and reinstatement), these data were not factored into the analyses.

The use of microdialysis as a sampling procedure for untargeted metabolomics analysis, as was done in the final experiment of the current research, is a novel approach and can provide important and relatively high-resolution temporal information. Further, microdialysis samples can be collected from awake, freely moving subjects, allowing for the concurrent collection of behavioural data. This provides the means to examine both metabolomic and behavioural changes with reasonable temporal resolution in response to acute drug or other stimulus exposure (e.g. stress, conditioned cues, etc.). Given appropriate statistical analyses, both timeseries metabolomics data, as well as behavioural data, can be used to help to identify metabolites that are the most relevant to the phenotype of interest (e.g. MDMA-produced behavioural sensitisation), as was demonstrated in the current research. Although the current procedures still needed further development, the current results do serve as a proof of concept. The current approach is unique not only in the addiction field, but also in other fields, and may be useful for examining both temporally and behaviourally relevant changes in the metabolome in response to various disease or treatment conditions.

\section{Limitations}

One possible limitation of the current research is that a yoked control group was not included in the $\Delta$ FosB study. This group would receive the same amount of i.v. MDMA exposure as MDMA self-administering rats, but non-contingently. This would allow any differences in $\triangle \mathrm{FosB}$ expression as a function of contingent vs non-contingent drug exposure to be examined. It is unsure whether this would have been expected to have much of an effect, however, based on previous studies. No differences in $\Delta \mathrm{FosB}$ accumulation in the NAcc core, shell, the C-P, or the medial PFC were observed in cocaine self-administering rats compared to yoked controls (Larson et al., 2010; Perrotti et al., 2008; Winstanley et al., 2007) with the only exception being a more pronounced increase in $\triangle \mathrm{FosB}$ accumulation in the OFC (Winstanley et al., 2007). 
It should also be mentioned that some of the experimental groups in the current research had a low sample size, notably within Chapters 2 and 3. While the sample sizes in Chapter 2 are indeed low, they are not uncommon and many comparable studies have had similar sample sizes (e.g. Cornish et al., 2012; Hope et al., 1994; Y. Kim et al., 2009; Perrotti et al., 2005; Pich et al., 1997). Importantly, the variation in both the independent (MDMA self-administration) and dependent $(\Delta \mathrm{FosB})$ variables is small, as is clearly shown in the figures. The effects are also large and significant in most cases, even after correction for multiple comparisons. In Chapter 3, some of the experimental groups also had a low samples size. Again, the variability in these data are small. The results are also supported by two different behavioural measures. Therefore, while it should still be acknowledged, this represents is a minor limitation.

Another possible limitation of the current research is the limited time periods at which neurochemical and behavioural data were collected. Behavioural sensitisation was assessed, and microdialysis samples were collected following 2 days of withdrawal from the MDMA pretreatment regimen. This time period was chosen in order to keep the procedures consistent and relevant to previous research where the same pretreatment regimen and withdrawal period was used to assess the effect of repeated MDMA exposure on the reinforcing effect of MDMA (van de Wetering \& Schenk, 2017). If such neurochemical and behavioural assays were carried out after longer withdrawal periods, it might be expected that both neurochemical and behavioural sensitisation would become more pronounced, as has been shown with other drugs of abuse (Kalivas \& Duffy, 1993b, 1993a; Paulson \& Robinson, 1991, 1995; Vanderschuren et al., 1999). It also might be expected that different neuroadaptations may develop with protracted withdrawal, which may also be of interest (Pierce \& Kalivas, 1997; Vanderschuren \& Kalivas, 2000; Wolf, 1998).

There are also some limitations related to the use of microdialysis as a sampling procedure, as was done in Chapters 4 and 5. The temporal resolution of microdialysis (minutes) is worse compared to in vivo fast-scan cyclic voltammetry (seconds), which can also be used to measure extracellular concentrations of target analytes (D. L. Robinson, Venton, Heien, \& Wightman, 2003). This procedure cannot be used to measure multiple analytes simultaneously, however, which would make it unsuitable for the current purposes. Another limitation of microdialysis is that very few brain regions can be sampled simultaneously. This was why the brain region sampled from in the current research was chosen based on the regions of interest identified in Chapters 2 and 3. Other procedures, such as IHC or tissue analysis, could be used to investigate multiple brain regions simultaneously. Of course, these other procedures have 
their own limitations, such as a complete lack of temporal resolution. These procedures do provide much higher concentrations of analytes, however, which would make the analysis of some low concentration compounds easier (e.g. 5-HT).

In Chapter 4, a procedure for the quantification of multiple neurochemical targets was developed given the available resources, equipment, and time. There were several limitations to this procedure, however, and several improvements could still be made. First, the use of D2 internal standards was not ideal due to the isotopic overlap with the target analytes. D5 or ${ }^{13} \mathrm{C}_{6}$ $\mathrm{BzCl}$ is widely available and could be used to derivatise regular chemical standards to produce physiochemically similar internal standards for most target analytes, as has been done previously (Song et al., 2012; Wong et al., 2016). This would prevent the need to purchase or produce separate internal standards for each target analyte and also prevent the need for any isotopic dilution calculations. Second, refinement of the sample and/or the mobile phase buffers might help optimise the $\mathrm{pH}$ conditions for the derivatisation, retention, and ionisation of more target analytes, such as DOPAC and 5-HIAA. Although these analytes were originally able to be quantified, they were not able to be reliably detected using the final procedures, which were ultimately optimised for the detection of 5-HT. Adjusting the buffers as well as the $\mathrm{BzCl}$ percentage used for derivatisation may also help with the stability of the target analytes such as DA (Song et al., 2012; Wong et al., 2016). Finally, the targeted analyses may have been better suited to a triple quadrupole MS (QQQ), which are generally more appropriate for the quantification of known target analytes than Q-TOF MS. QQQs have greater analytical sensitivity and would have less trouble detecting and quantifying the low concentration of 5HT. Additionally, QQQs have a greater linear range, which may have prevented the issues in the current study where concentrations of 5-HT were close to the detection limits, but the concentrations of MDMA were too high and saturating the MS detector. It should be noted that Q-TOF MS has very accurate mass detection, however, and are the preferred instrument for untargeted approaches such as those carried out in Chapter 5.

There are also some limitations and improvements that could be made to the untargeted metabolomics procedures. First and foremost, the extensive optimisation of the sample preparation and analytical procedures, as was carried out in Chapter 4, was focused on the targeted quantification a few metabolites, namely 5-HT, and not untargeted metabolomics. As a result, the 'metabolomic net' that was cast with the current procedures was very small. Numerous metabolites that may have been of interest that should be detected within benzoylated microdialysis samples such as glutamate or GABA (Song et al., 2012; Wong et 
al., 2016), were not detected in the current samples. Given more inclusive sample preparation and HPLC methods, more metabolites could have been detected and analysed, and a more comprehensive analysis of the neuro-metabolome could have been carried out. Second, although $\mathrm{BzCl}$ has several advantages for targeted procedures (Song et al., 2012; Wong et al., 2016), it does make the identification of unknown metabolites more complicated, since there are no databases of benzoylated metabolites to compare the results with. Lastly, there was sizeable uninduced variation in the metabolomic profile in the current study. This was evidenced by the results of the PCA and the large orthogonal separation in the OPLS models. Interestingly, these models separated samples that were analysed prior to, and following, a lengthy period of LC-MS maintenance and sample storage at $-80^{\circ} \mathrm{C}$, which may have been the cause for the loss of the DA data in Chapter 4. These results suggested that the majority of the variation in the data was due to variations in the sample preparation, storage, or analytical procedures. Thankfully, the statistical models used in the current procedure are capable of dealing with such variation.

\section{Future directions}

One of the most interesting findings of the current research was the region-specific increases in $\triangle \mathrm{F} O \mathrm{OBB}$ in the striatum following MDMA self-administration. Significant increases in $\Delta$ FosB were observed in brain regions that are known to be involved in goal-directed behaviour, but not in brain regions that have been implicated in stimulus-driven behaviour (i.e. habitual behaviour). This might suggest one of two things; either $\Delta$ FosB does not mediate neuroadaptive responses related to the development of habitual behaviour or that MDMA selfadministration in the current research was not habitual.

The latter has not been directly assessed and could be investigated with the use of reward devaluation procedures. A significant decrease in MDMA-taking or seeking behaviour following reward devaluation would suggest that the behaviour is not habitual, but still goaldriven. In the current study, over 29 sessions of 6-hour MDMA self-administration were carried out, with approximately 1300 reinforced responses made per subject. Given that habitual selfadministration of other drugs of abuse has been demonstrated with lesser amounts of training (Clemens et al., 2014; Corbit et al., 2012; Dickinson et al., 2002; Miles et al., 2003; Olmstead et al., 2001; Zapata et al., 2010), it would be expected that MDMA self-administration in the current study would be similarly habitual.

If this were indeed the case, then the current results would suggest that $\Delta$ FosB does not mediate neuroadaptive responses related to the development of habitual behaviour. $\Delta \mathrm{FosB}$ is 
capable of mediating neuroadaptive changes within these brain regions that would be expected to influence both goal-directed and habitual behaviour, however (Nestler, 2008; Robison \& Nestler, 2011). Thus, to elucidate the role of $\Delta$ FosB in the development of habitual drugtaking/seeking behaviour, future research could correlate $\Delta$ FosB accumulation in these brain regions with the degree to which subjects are susceptible to reward devaluation following drug self-administration. Alternatively, the effect of selective overexpression of $\Delta \mathrm{FosB}$ in these brain regions on habitual drug-taking or seeking behaviour, as assessed by reward devaluation, could be examined.

Another valuable avenue for future research would be to continue the development of the untargeted metabolomics procedures and apply them to identify both behaviourally and/or temporally relevant changes in the metabolome that are associated with various treatment or disease conditions. Regarding drug addiction, these procedures could be used to identify metabolites that are relevant to drug-seeking responses, for example, or to identify relevant differences in the metabolite profile of drug self-administering subjects versus yoked controls. Because untargeted metabolomics is a relatively novel analytical approach in the addiction field, there are multiple interesting avenues for future research using such procedures.

Further optimisation of the procedures would be needed, however, particularly to the sample preparation and analysis procedures. As previously mentioned, this would include the optimisation of the sample and mobile phase buffers, the percentage of $\mathrm{BzCl}$ used for derivatisation, the chromatography gradient, and the type of column used in order to increase the number of metabolites able to detected and analysed. This would provide a much more comprehensive analysis and the casting of a much larger 'metabolomic net'. Another improvement could be to use more than one internal standard. D2 5-HT may not be a useful internal standard for some metabolites with different physiochemical properties. D5 or ${ }^{13} \mathrm{C}_{6}$ $\mathrm{BzCl}$ could be used to make a range of internal standards to provide better normalisation methods for a wider range of diverse metabolites (Sysi-Aho et al., 2007). Improvements to the data analysis procedure could also be made. For example, subtracting the response from blank samples (aCSF) from the dialysate samples and including more replicates may help to clean up the data. Further, the adducts and in-source fragments of highly abundant compounds could be identified and filtered prior to statistical analysis. This could be achieved using MZmine and algorithms such as CAMERA (Kuhl, Tautenhahn, Böttcher, Larson, \& Neumann, 2012) or with subsequent time-series cluster analyses (Rusilowicz et al., 2018). 


\section{Conclusions}

Drug addiction is a chronic, relapsing disorder characterised by compulsive drugseeking and drug-taking behaviour despite adverse consequences. Drug addiction exacts an enormous medical, financial, and emotional toll on society and there is an unmet need for new prevention and treatment strategies. In order to develop these strategies, however, a better understanding of the various factors underlying harmful drug use and the development of SUDs are required.

Given the persistent and relapsing nature of SUDs, an important focus of addiction research recently has been to investigate the mechanisms by which maladaptive, drug-induced neuroadaptations are maintained over such long periods of time, even after drug exposure has ceased. Recent evidence has highlighted the important role of the transcription factor, $\Delta \mathrm{FosB}$, in mediating the development of such persistent drug-induced neuroadaptations involved in perpetuating addictive behaviour (Robison \& Nestler, 2011). The findings of the current research add to this growing body of evidence, indicating that repeated, chronic exposure to MDMA, like other drugs of abuse, induces a persistent accumulation of $\triangle \mathrm{FosB}$ in several brain regions implicated in addictive behaviour. Future research may extend these fundamental findings to develop novel treatments for addiction that target transcriptional-related mechanisms such as $\Delta \mathrm{FosB}$. As has been previously suggested, the presence of $\Delta \mathrm{FosB}$ in certain brain regions may also prove to be a useful marker of addiction and could be used for monitoring the progress of treatment in the future (Nestler, 2008).

The results of the current thesis suggest that repeated MDMA exposure induces many of the same neuroadaptations that result from repeated exposure to other drugs of abuse. These include increases in levels of $\Delta \mathrm{FosB}$ in several relevant brain regions as well as potential changes in DAergic mechanisms. It has been suggested that MDMA self-administration progresses as a function of neuroadaptive responses in the same brain regions that mediate the progression of self-administration of other drugs of abuse (Schenk, 2011). The present data are consistent with this idea. The present data also support the idea that repeated MDMA exposure induces neuroadaptations that render both the pharmacological and behavioural profile of MDMA increasingly similar to that of other psychostimulants such as methamphetamine (Schenk, 2011). This results in a drug that has far greater reinforcing efficacy (van de Wetering $\&$ Schenk, 2017), and thus, greater abuse liability with repeated use. This has important implications given the popularity of MDMA as a recreational drug of abuse and the more recent use of MDMA as a pharmacological adjunct to post-traumatic stress disorder psychotherapy. 
Finally, the application of untargeted metabolomics in the addiction field has exciting potential as a method for generating new research avenues and identifying novel therapeutic targets. The research carried out in this thesis represents the first step of this process. The use of microdialysis as a sampling procedure combined with sophisticated multivariate statistical methods allows for the identification of neurochemicals that are both temporally and behaviourally relevant to changes in disease state. This novel approach may also be of value for other preclinical research disciplines and certainly warrants future development. 


\section{References}

Aarde, S. M., Miller, M. L., Creehan, K. M., Vandewater, S. A., \& Taffe, M. A. (2015). One day access to a running wheel reduces self-administration of D-methamphetamine, MDMA and methylone. Drug and Alcohol Dependence, 151, 151-158. https://doi.org/10.1016/j.drugalcdep.2015.03.016

Abrahao, K. P., Quadros, I. M. H., \& Souza-Formigoni, M. L. O. (2011). Nucleus accumbens dopamine D1 receptors regulate the expression of ethanol-induced behavioural sensitization. The International Journal of Neuropsychopharmacology, 14(02), 175-185. https://doi.org/10.1017/S1461145710000441

Adams, C. D. (1982). Variations in the Sensitivity of Instrumental Responding to Reinforcer Devaluation. The Quarterly Journal of Experimental Psychology Section B, 34(2b), 7798. https://doi.org/10.1080/14640748208400878

Aharonovich, E., Hasin, D. S., Brooks, A. C., Liu, X., Bisaga, A., \& Nunes, E. V. (2006). Cognitive deficits predict low treatment retention in cocaine dependent patients. Drug and Alcohol Dependence, 81(3), 313-322. https://doi.org/10.1016/j.drugalcdep.2005.08.003

Ahmed, S. H. (2011). Escalation of Drug Use (pp. 267-292). Humana Press. https://doi.org/10.1007/978-1-60761-934-5_10

Ahmed, S. H., \& Koob, G. F. (1998). Transition from Moderate to Excessive Drug Intake: Change in Hedonic Set Point. Science, 282(5387), 298-300. https://doi.org/10.1126/science.282.5387.298

Ahmed, S. H., \& Koob, G. F. (2005). Transition to drug addiction: a negative reinforcement model based on an allostatic decrease in reward function. Psychopharmacology, 180(3), 473-490. https://doi.org/10.1007/s00213-005-2180-z

Ahmed, S. H., Walker, J. R., \& Koob, G. F. (2000). Persistent Increase in the Motivation to Take Heroin in Rats with a History of Drug Escalation. Neuropsychopharmacology, 22(4), 413-421. https://doi.org/10.1016/S0893-133X(99)00133-5

Alderson, H. L., Robbins, T. W., \& Everitt, B. J. (2000). The effects of excitotoxic lesions of the basolateral amygdala on the acquisition of heroin-seeking behaviour in rats. Psychopharmacology, 153(1), 111-119. https://doi.org/10.1007/s002130000527

Alibhai, I. N., Green, T. A., Potashkin, J. A., \& Nestler, E. J. (2007). Regulation of fosB and $\Delta$ fosB mRNA expression: In vivo and in vitro studies. Brain Research, 1143, 22-33. https://doi.org/10.1016/J.BRAINRES.2007.01.069

Almeida, A. ., Castel-Branco, M. ., \& Falcão, A. . (2002). Linear regression for calibration lines revisited: weighting schemes for bioanalytical methods. Journal of Chromatography B, 774(2), 215-222. https://doi.org/10.1016/S1570-0232(02)00244-1

Álvarez-Sánchez, B., Priego-Capote, F., \& Castro, M. D. L. de. (2010). Metabolomics analysis II. Preparation of biological samples prior to detection. TrAC Trends in Analytical Chemistry, 29(2), 120-127. https://doi.org/10.1016/J.TRAC.2009.12.004

Álvarez-Sánchez, B., Priego-Capote, F., \& Luque de Castro, M. D. (2010). Metabolomics analysis I. Selection of biological samples and practical aspects preceding sample preparation. TrAC Trends in Analytical Chemistry, 29(2), 111-119. https://doi.org/10.1016/J.TRAC.2009.12.003

American Psychiatric Association [APA]. (2013). DSM-V. Diagnostic and Statistical Manual of Mental Disorders (5th ed.). APA Publishing.

Ang, E., Chen, J., Zagouras, P., Magna, H., Holland, J., Schaeffer, E., \& Nestler, E. J. (2008). Induction of nuclear factor- $\kappa \mathrm{B}$ in nucleus accumbens by chronic cocaine administration. Journal of Neurochemistry, 79(1), 221-224. https://doi.org/10.1046/j.14714159.2001.00563.x

Antti, H., Bollard, M. E., Ebbels, T. M., Keun, H., Lindon, J. C., Nicholson, J. K., \& Holmes, 
E. (2002). Batch statistical processing of1H NMR-derived urinary spectral data. Journal of Chemometrics, 16(8-10), 461-468. https://doi.org/10.1002/cem.733

Araujo, P. (2009). Key aspects of analytical method validation and linearity evaluation. Journal of Chromatography $\quad B, \quad$ 877(23), $2224-2234$. https://doi.org/10.1016/J.JCHROMB.2008.09.030

Armbruster, D. A., \& Pry, T. (2008). Limit of blank, limit of detection and limit of quantitation. The Clinical Biochemist. Reviews, 29 Suppl 1(Suppl 1), S49-52. Retrieved from http://www.ncbi.nlm.nih.gov/pubmed/18852857

Ator, N. A., \& Griffiths, R. R. (2003). Principles of drug abuse liability assessment in laboratory animals. Drug and Alcohol Dependence, 70(3), S55-S72. https://doi.org/10.1016/S0376-8716(03)00099-1

Baldo, B. A., \& Kelley, A. E. (2007). Discrete neurochemical coding of distinguishable motivational processes: insights from nucleus accumbens control of feeding. Psychopharmacology, 191(3), 439-459. https://doi.org/10.1007/s00213-007-0741-z

Ball, K. T., Budreau, D., \& Rebec, G. V. (2003). Acute effects of 3,4methylenedioxymethamphetamine on striatal single-unit activity and behavior in freely moving rats: differential involvement of dopamine D1 and D2 receptors. Brain Research, 994(2), 203-215. https://doi.org/10.1016/j.brainres.2003.09.037

Ball, K. T., Budreau, D., \& Rebec, G. V. (2006). Context-dependent behavioural and neuronal sensitization in striatum to MDMA (ecstasy) administration in rats. The European Journal of Neuroscience, 24(1), 217-228. https://doi.org/10.1111/j.1460-9568.2006.04885.x

Ball, K. T., Jarsocrak, H., Hyacinthe, J., Lambert, J., Lockowitz, J., \& Schrock, J. (2015). Yohimbine reinstates extinguished 3,4-methylenedioxymethamphetamine (MDMA; ecstasy) seeking in rats with prior exposure to chronic yohimbine. Behavioural Brain Research. https://doi.org/10.1016/j.bbr.2015.07.056

Ball, K. T., Klein, J. E., Plocinski, J. A., \& Slack, R. (2011). Behavioral sensitization to 3,4methylenedioxymethamphetamine is long-lasting and modulated by the context of drug administration. Behavioural Pharmacology, 22(8), 847-850. https://doi.org/10.1097/FBP.0b013e32834d13b4

Ball, K. T., \& Slane, M. (2012). Differential involvement of prelimbic and infralimbic medial prefrontal cortex in discrete cue-induced reinstatement of 3,4methylenedioxymethamphetamine (MDMA; ecstasy) seeking in rats. Psychopharmacology, 224(3), 377-385. https://doi.org/10.1007/s00213-012-2762-5

Ball, K. T., \& Slane, M. (2014). Tolerance to the locomotor-activating effects of 3,4methylenedioxymethamphetamine (MDMA) predicts escalation of MDMA selfadministration and cue-induced reinstatement of MDMA seeking in rats. Behavioural Brain Research, 274, 143-148. https://doi.org/10.1016/j.bbr.2014.08.010

Ball, K. T., Walsh, K. M., \& Rebec, G. V. (2007). Reinstatement of MDMA (ecstasy) seeking by exposure to discrete drug-conditioned cues. Pharmacology, Biochemistry, and Behavior, 87(4), 420-425. https://doi.org/10.1016/j.pbb.2007.05.018

Ball, K. T., Wellman, C. L., Fortenberry, E., \& Rebec, G. V. (2009). Sensitizing regimens of $( \pm) 3$, 4-methylenedioxymethamphetamine (ecstasy) elicit enduring and differential structural alterations in the brain motive circuit of the rat. Neuroscience, 160(2), 264-274. https://doi.org/10.1016/J.NEUROSCIENCE.2009.02.025

Balleine, B. W., \& O’Doherty, J. P. (2010). Human and Rodent Homologies in Action Control: Corticostriatal Determinants of Goal-Directed and Habitual Action. Neuropsychopharmacology, 35(1), 48-69. https://doi.org/10.1038/npp.2009.131

Balster, R. L., Kilbey, M. M., \& Ellinwood, E. H. (1976). Methamphetamine selfadministration in the cat. Psychopharmacologia, 46(3), 229-233. https://doi.org/10.1007/BF00421107 
Banks, M. L., Czoty, P. W., Gage, H. D., Bounds, M. C., Garg, P. K., Garg, S., \& Nader, M. A. (2008). Effects of cocaine and MDMA self-administration on serotonin transporter availability in monkeys. Neuropsychopharmacology: Official Publication of the American College of Neuropsychopharmacology, 33(2), 219-225. https://doi.org/10.1038/sj.npp.1301420

Bankson, M. G., \& Cunningham, K. A. (2001). 3,4-Methylenedioxymethamphetamine (MDMA) as a Unique Model of Serotonin Receptor Function and Serotonin-Dopamine Interactions. J. Pharmacol. Exp. Ther., 297(3), 846-852. Retrieved from http://jpet.aspetjournals.org/content/297/3/846.short

Barnes, N. M., \& Sharp, T. (1999). A review of central 5-HT receptors and their function. Neuropharmacology, 38(8), 1083-1152. https://doi.org/10.1016/S0028-3908(99)000106

Barrett, A. C., Miller, J. R., Dohrmann, J. M., \& Caine, S. B. (2004). Effects of dopamine indirect agonists and selective D1-like and D2-like agonists and antagonists on cocaine self-administration and food maintained responding in rats. Neuropharmacology, 47 Suppl 1, 256-273. https://doi.org/10.1016/j.neuropharm.2004.07.007

Bartel, J., Krumsiek, J., \& Theis, F. J. (2013). STATISTICAL METHODS FOR THE ANALYSIS OF HIGH-THROUGHPUT METABOLOMICS DATA. Computational and Structural Biotechnology Journal, 4(5), e201301009. https://doi.org/10.5936/CSBJ.201301009

Bassareo, V., De Luca, M. A., \& Di Chiara, G. (2002). Differential Expression of Motivational Stimulus Properties by Dopamine in Nucleus Accumbens Shell versus Core and Prefrontal Cortex. The Journal of Neuroscience: The Official Journal of the Society for Neuroscience, 22(11), 4709-4719. https://doi.org/20026445

Bassareo, V., \& Di Chiara, G. (1997). Differential influence of associative and nonassociative learning mechanisms on the responsiveness of prefrontal and accumbal dopamine transmission to food stimuli in rats fed ad libitum. The Journal of Neuroscience: The Official Journal of the Society for Neuroscience, 17(2), 851-861. https://doi.org/10.1523/JNEUROSCI.17-02-00851.1997

Bassareo, V., \& Di Chiara, G. (1999a). Differential responsiveness of dopamine transmission to food-stimuli in nucleus accumbens shell/core compartments. Neuroscience, 89(3), 637641. https://doi.org/10.1016/S0306-4522(98)00583-1

Bassareo, V., \& Di Chiara, G. (1999b). Modulation of feeding-induced activation of mesolimbic dopamine transmission by appetitive stimuli and its relation to motivational state. European Journal of Neuroscience, 11(12), 4389-4397. https://doi.org/10.1046/j.1460-9568.1999.00843.x

Battaglia, G., Brooks, B. P., Kulsakdinun, C., \& De Souza, E. B. (1988). Pharmacologic profile of MDMA (3,4-methylenedioxymethamphetamine) at various brain recognition sites. European Journal of Pharmacology, 149(1-2), 159-163. https://doi.org/10.1016/00142999(88)90056-8

Battaglia, G., Yeh, S. Y., \& De Souza, E. B. (1988). MDMA-induced neurotoxicity: parameters of degeneration and recovery of brain serotonin neurons. Pharmacology, Biochemistry, and Behavior, 29(2), 269-274. Retrieved from http://www.ncbi.nlm.nih.gov/pubmed/2452449

Battaglia, G., Yeh, S. Y., O’Hearn, E., Molliver, M. E., Kuhar, M. J., \& De Souza, E. B. (1987). 3,4-Methylenedioxymethamphetamine and 3,4-methylenedioxyamphetamine destroy serotonin terminals in rat brain: quantification of neurodegeneration by measurement of [3H]paroxetine-labeled serotonin uptake sites. J. Pharmacol. Exp. Ther., 242(3), 911916. Retrieved from http://jpet.aspetjournals.org/content/242/3/911.short

Baumann, M. H., Clark, R. D., Franken, F. H., Rutter, J. J., \& Rothman, R. B. (2008). Tolerance 
to 3,4-methylenedioxymethamphetamine in rats exposed to single high-dose binges. Neuroscience, 152(3), 773-784. https://doi.org/10.1016/j.neuroscience.2008.01.007

Baumann, M. H., Clark, R. D., \& Rothman, R. B. (2008). Locomotor stimulation produced by 3,4-methylenedioxymethamphetamine (MDMA) is correlated with dialysate levels of serotonin and dopamine in rat brain. Pharmacology, Biochemistry, and Behavior, 90(2), 208-217. https://doi.org/10.1016/j.pbb.2008.02.018

Baumann, M. H., Wang, X., \& Rothman, R. B. (2007). 3,4-Methylenedioxymethamphetamine (MDMA) neurotoxicity in rats: a reappraisal of past and present findings. Psychopharmacology, 189(4), 407-424. https://doi.org/10.1007/s00213-006-0322-6

Baumann, M. H., Zolkowska, D., Kim, I., Scheidweiler, K. B., Rothman, R. B., \& Huestis, M. A. (2009). Effects of dose and route of administration on pharmacokinetics of (+ or -)3,4-methylenedioxymethamphetamine in the rat. Drug Metabolism and Disposition: The Biological Fate of Chemicals, 37(11), 2163-2170. https://doi.org/10.1124/dmd.109.028506

Beardsley, P. M., Balster, R. L., \& Harris, L. S. (1986). Self-administration of methylenedioxymethamphetamine (MDMA) by rhesus monkeys. Drug and Alcohol Dependence, 18(2), 149-157. https://doi.org/10.1016/0376-8716(86)90047-5

Belin, D., Berson, N., Balado, E., Piazza, P. V., \& Deroche-Gamonet, V. (2011). HighNovelty-Preference Rats are Predisposed to Compulsive Cocaine Self-administration. Neuropsychopharmacology, 36(3), 569-579. https://doi.org/10.1038/npp.2010.188

Belin, D., Mar, A. C., Dalley, J. W., Robbins, T. W., \& Everitt, B. J. (2008). High Impulsivity Predicts the Switch to Compulsive Cocaine-Taking. Science, 320(5881), 1352-1355. https://doi.org/10.1126/science.1158136

Belin, M. F., Aguera, M., Tappaz, M., McRae-Degueurce, A., Bobillier, P., \& Pujol, J. F. (1979). GABA-accumulating neurons in the nucleus raphe dorsalis and periaqueductal gray in the rat: A biochemical and radioautographic study. Brain Research, 170(2), 279297. https://doi.org/10.1016/0006-8993(79)90107-0

Belin, M. F., Nanopoulos, D., Didier, M., Aguera, M., Steinbusch, H., Verhofstad, A., ... Pujol, J. F. (1983). Immunohistochemical evidence for the presence of $\gamma$-aminobutyric acid and serotonin in one nerve cell. A study on the raphe nuclei of the rat using antibodies to glutamate decarboxylase and serotonin. Brain Research, 275(2), 329-339. https://doi.org/10.1016/0006-8993(83)90994-0

Benamar, K., Geller, E. B., \& Adler, M. W. (2008). A new brain area affected by 3,4methylenedioxymethamphetamine: A microdialysis-biotelemetry study. European Journal of Pharmacology, 596(1-3), 84-88. https://doi.org/10.1016/J.EJPHAR.2008.08.001

Bentley, T. W., Carter, G. E., \& Harris, H. C. (1984). SN 2 character of hydrolysis of benzoyl chloride. Journal of the Chemical Society, Chemical Communications, (6), 387-389.

Berger, U. V., Gu, X. F., \& Azmitia, E. C. (1992). The substituted amphetamines 3,4methylenedioxymethamphetamine, methamphetamine, p-chloroamphetamine and fenfluramine induce 5-hydroxytryptamine release via a common mechanism blocked by fluoxetine and cocaine. European Journal of Pharmacology, 215(2-3), 153-160. https://doi.org/10.1016/0014-2999(92)90023-W

Berridge, K. C., \& Kringelbach, M. L. (2015). Pleasure systems in the brain. Neuron, 86(3), 646-664. https://doi.org/10.1016/j.neuron.2015.02.018

Berridge, K. C., \& Robinson, T. E. (2016). Liking, wanting, and the incentive-sensitization theory of addiction. The American Psychologist, 71(8), 670-679. https://doi.org/10.1037/amp0000059

Berridge, K. C., \& Valenstein, E. S. (1991). What psychological process mediates feeding evoked by electrical stimulation of the lateral hypothalamus? Behavioral Neuroscience, 
105(1), 3-14. https://doi.org/10.1037//0735-7044.105.1.3

Berridge, K. C., Venier, I. L., \& Robinson, T. E. (1989). Taste reactivity analysis of 6hydroxydopamine-induced aphagia: implications for arousal and anhedonia hypotheses of dopamine function. Behavioral Neuroscience, 103(1), 36-45. https://doi.org/10.1037//0735-7044.103.1.36

Berry, M. D., Gainetdinov, R. R., Hoener, M. C., \& Shahid, M. (2017). Pharmacology of human trace amine-associated receptors: Therapeutic opportunities and challenges. Pharmacology $\& \quad$ Therapeutics, 161-180. https://doi.org/10.1016/J.PHARMTHERA.2017.07.002

Bexis, S., \& Docherty, J. R. (2006). Effects of MDMA, MDA and MDEA on blood pressure, heart rate, locomotor activity and body temperature in the rat involve $\alpha$-adrenoceptors. British Journal of Pharmacology, 147(8), 926-934. https://doi.org/10.1038/sj.bjp.0706688

Bibb, J. A., Chen, J., Taylor, J. R., Svenningsson, P., Nishi, A., Snyder, G. L., ... Greengard, P. (2001). Effects of chronic exposure to cocaine are regulated by the neuronal protein Cdk5. Nature, 410(6826), 376-380. https://doi.org/10.1038/35066591

Bindra, D. (1974). A motivational view of learning, performance, and behavior modification. Psychological Review, 81(3), 199-213. https://doi.org/10.1037/h0036330

Bindra, D. (1978). How adaptive behavior is produced: a perceptual-motivational alternative to response reinforcements. Behavioral and Brain Sciences, 1(1), 41-52. https://doi.org/10.1017/S0140525X00059380

Bird, J., \& Schenk, S. (2013). Contribution of impulsivity and novelty-seeking to the acquisition and maintenance of MDMA self-administration. Addiction Biology, 18(4), 654-664. https://doi.org/10.1111/j.1369-1600.2012.00477.x

Bishop, M. P., Elder, S. T., \& Heath, R. G. (1963). Intracranial self-stimulation in man. Science, 140(3565), 394-396.

Boccard, J., \& Rudaz, S. (2014). Harnessing the complexity of metabolomic data with chemometrics. Journal of Chemometrics, 28(1), 1-9. https://doi.org/10.1002/cem.2567

Bogen, I. L., Haug, K. H., Myhre, O., \& Fonnum, F. (2003). Short- and long-term effects of MDMA ("ecstasy") on synaptosomal and vesicular uptake of neurotransmitters in vitro and ex vivo. Neurochemistry International, 43(4-5), 393-400. https://doi.org/10.1016/S0197-0186(03)00027-5

Bolles, R. C. (1972). Reinforcement, expectancy, and learning. Psychological Review, 79(5), 394-409. https://doi.org/10.1037/h0033120

Bossé, G. D., \& Peterson, R. T. (2017). Development of an opioid self-administration assay to study drug seeking in zebrafish. Behavioural Brain Research, 335, 158-166. https://doi.org/10.1016/J.BBR.2017.08.001

Bozarth, M. A. (1987). Intracranial Self-Administration Procedures for the Assessment of Drug Reinforcement. In Methods of Assessing the Reinforcing Properties of Abused Drugs (pp. 173-187). New York, NY: Springer New York. https://doi.org/10.1007/978-1-46124812-5 9

Bozarth, M. A., \& Wise, R. A. (1981). Intracranial self-administration of morphine into the ventral tegmental area in rats. Life Sciences, 28(5), 551-555. https://doi.org/10.1016/0024-3205(81)90148-X

Bozarth, M. A., \& Wise, R. A. (1982). Localization of the reward-relevant opiate receptors. Problems of Drug Dependence, 158.

Bozarth, M. A., \& Wise, R. A. (1985). Toxicity associated with long-term intravenous heroin and cocaine self-administration in the rat. JAMA, 254(1), 81-83. Retrieved from http://www.ncbi.nlm.nih.gov/pubmed/4039767

Bozarth, M. A., \& Wise, R. A. (1986). Involvement of the ventral tegmental dopamine system 
in opioid and psychomotor stimulant reinforcement. NIDA Res Monogr, 67, 190-196.

Bradbury, S., Bird, J., Colussi-Mas, J., Mueller, M., Ricaurte, G. A., \& Schenk, S. (2013). Acquisition of MDMA self-administration: pharmacokinetic factors and MDMA-induced serotonin release. Addict Biol, 19(5), 874-884. https://doi.org/10.1111/adb.12069

Bradbury, S., Gittings, D., \& Schenk, S. (2012). Repeated exposure to MDMA and amphetamine: sensitization, cross-sensitization, and response to dopamine $\mathrm{D}_{1}$ - and $\mathrm{D}_{2}$ like agonists. Psychopharmacology, 223(4), 389-399. https://doi.org/10.1007/s00213012-2726-9

Brauer, L. H., \& de Wit, H. (1997). High dose pimozide does not block amphetamine-induced euphoria in normal volunteers. Pharmacology, Biochemistry, and Behavior, 56(2), 265272. https://doi.org/10.1016/s0091-3057(96)00240-7

Brenhouse, H. C., \& Stellar, J. R. (2006). c-Fos and $\Delta$ FosB expression are differentially altered in distinct subregions of the nucleus accumbens shell in cocaine-sensitized rats. Neuroscience, $137(3)$, $773-780$. https://doi.org/10.1016/J.NEUROSCIENCE.2005.09.039

Brennan, K. A., Carati, C., Lea, R. A., Fitzmaurice, P. S., \& Schenk, S. (2009). Effect of D1like and D2-like receptor antagonists on methamphetamine and 3, 4methylenedioxymethamphetamine self-administration in rats. Behavioural Pharmacology, 20(8), 688-694.

Brennan, K. A., \& Schenk, S. (2006). Initial deficit and recovery of function after MDMA preexposure in rats. Psychopharmacology, 184(2), 239-246. https://doi.org/10.1007/s00213-005-0278-y

Briand, L. A., Flagel, S. B., Garcia-Fuster, M. J., Watson, S. J., Akil, H., Sarter, M., \& Robinson, T. E. (2008). Persistent Alterations in Cognitive Function and Prefrontal Dopamine D2 Receptors Following Extended, but Not Limited, Access to SelfAdministered Cocaine. Neuropsychopharmacology, 33(12), 2969-2980. https://doi.org/10.1038/npp.2008.18

Briand, L. A., Gross, J. P., \& Robinson, T. E. (2008). Impaired object recognition following prolonged withdrawal from extended-access cocaine self-administration. Neuroscience, 155(1), 1-6. https://doi.org/10.1016/j.neuroscience.2008.06.004

Britton, D. R., Curzon, P., Mackenzie, R. G., Kebabian, J. W., Williams, J. E. G., \& Kerkman, D. (1991). Evidence for involvement of both D1 and D2 receptors in maintaining cocaine self-administration. Pharmacology Biochemistry and Behavior, 39(4), 911-915. https://doi.org/10.1016/0091-3057(91)90052-4

Broadhurst, D. I., \& Kell, D. B. (2007). Statistical strategies for avoiding false discoveries in metabolomics and related experiments. Metabolomics, 2(4), 171-196. https://doi.org/10.1007/s11306-006-0037-z

Brown, R. W., \& Kolb, B. (2001). Nicotine sensitization increases dendritic length and spine density in the nucleus accumbens and cingulate cortex. Brain Research, 899(1-2), 94100. https://doi.org/10.1016/S0006-8993(01)02201-6

Bruchas, M. R., Land, B. B., \& Chavkin, C. (2010). The dynorphin/kappa opioid system as a modulator of stress-induced and pro-addictive behaviors. Brain Research, 1314, 44-55. https://doi.org/10.1016/J.BRAINRES.2009.08.062

Bubar, M. J., Pack, K. M., Frankel, P. S., \& Cunningham, K. A. (2004). Effects of dopamine D1- or D2-like receptor antagonists on the hypermotive and discriminative stimulus effects of (+)-MDMA. Psychopharmacology, 173(3-4), 326-336. https://doi.org/10.1007/s00213-004-1790-1

Bunzow, J. R., Sonders, M. S., Arttamangkul, S., Harrison, L. M., Zhang, G., Quigley, D. I., ... Grandy, D. K. (2001). Amphetamine, 3,4-Methylenedioxymethamphetamine, Lysergic Acid Diethylamide, and Metabolites of the Catecholamine Neurotransmitters 
Are Agonists of a Rat Trace Amine Receptor. Molecular Pharmacology, 60(6), 11811188. https://doi.org/10.1124/mol.60.6.1181

Bylesjö, M., Rantalainen, M., Cloarec, O., Nicholson, J. K., Holmes, E., \& Trygg, J. (2006). OPLS discriminant analysis: combining the strengths of PLS-DA and SIMCA classification. Journal of Chemometrics, 20(8-10), 341-351. https://doi.org/10.1002/cem.1006

Cador, M., Bjijou, Y., \& Stinus, L. (1995). Evidence of a complete independence of the neurobiological substrates for the induction and expression of behavioral sensitization to amphetamine. Neuroscience, 65(2), 385-395. https://doi.org/10.1016/03064522(94)00524-9

Caggiula, A., Donny, E., White, A., Chaudhri, N., Booth, S., Gharib, M., ... Sved, A. (2002). Environmental stimuli promote the acquisition of nicotine self-administration in rats. Psychopharmacology, 163(2), 230-237. https://doi.org/10.1007/s00213-002-1156-5

Caine, S. B., Heinrichs, S. C., Coffin, V. L., \& Koob, G. F. (1995). Effects of the dopamine D1 antagonist SCH 23390 microinjected into the accumbens, amygdala or striatum on cocaine self-administration in the rat. Brain Research, 692(1-2), 47-56. https://doi.org/10.1016/0006-8993(95)00598-k

Caine, S. B., \& Koob, G. F. (1994a). Effects of dopamine D-1 and D-2 antagonists on cocaine self- administration under different schedules of reinforcement in the rat. J. Pharmacol. Exp. Ther., 270(1), 209-218. Retrieved from http://jpet.aspetjournals.org/content/270/1/209.short

Caine, S. B., \& Koob, G. F. (1994b). Effects of mesolimbic dopamine depletion on responding maintained by cocaine and food. Journal of the Experimental Analysis of Behavior, 61(2), 213-221. https://doi.org/10.1901/jeab.1994.61-213

Callaway, C. W., Rempel, N. L., Peng, R. Y., \& Geyer, M. A. (1992). Serotonin 5-HT1-like receptors mediate hyperactivity in rats induced by 3,4-methylenedioxymethamphetamine. Neuropsychopharmacology.

Callaway, C. W., Wing, L. L., \& Geyer, M. A. (1990). Serotonin release contributes to the locomotor stimulant effects of 3,4- methylenedioxymethamphetamine in rats. $J$. Pharmacol. Exp. Ther., 254(2), 456-464. Retrieved from http://jpet.aspetjournals.org/content/254/2/456.short

Calu, D. J., Stalnaker, T. A., Franz, T. M., Singh, T., Shaham, Y., \& Schoenbaum, G. (2007). Withdrawal from cocaine self-administration produces long-lasting deficits in orbitofrontal-dependent reversal learning in rats. Learning \& Memory, 14(5), 325-328. https://doi.org/10.1101/lm.534807

Camarini, R., \& Hodge, C. W. (2004). Ethanol preexposure increases ethanol selfadministration in C57BL/6J and DBA/2J mice. Pharmacology, Biochemistry, and Behavior, 79(4), 623-632. https://doi.org/10.1016/j.pbb.2004.09.012

Canales, J. J. (2005). Stimulant-induced adaptations in neostriatal matrix and striosome systems: Transiting from instrumental responding to habitual behavior in drug addiction. Neurobiology of Learning and Memory, 83(2), 93-103. https://doi.org/10.1016/j.nlm.2004.10.006

Capriles, N., Rodaros, D., Sorge, R. E., \& Stewart, J. (2003). A role for the prefrontal cortex in stress- and cocaine-induced reinstatement of cocaine seeking in rats. Psychopharmacology, 168(1-2), 66-74. https://doi.org/10.1007/s00213-002-1283-z

Carle, T. L., Ohnishi, Y. N., Ohnishi, Y. H., Alibhai, I. N., Wilkinson, M. B., Kumar, A., \& Nestler, E. J. (2007). Proteasome-dependent and -independent mechanisms for FosB destabilization: identification of FosB degron domains and implications for $\Delta$ FosB stability. European Journal of Neuroscience, 25(10), 3009-3019. https://doi.org/10.1111/j.1460-9568.2007.05575.x 
Carlsson, A., Lindqvist, M., Magnusson, T., \& Waldeck, B. (1958). On the presence of 3hydroxytyramine in brain. Science, $127,471$.

Carr, G. D., \& White, N. M. (1987). Effects of systemic and intracranial amphetamine injections on behavior in the open field: A detailed analysis. Pharmacology Biochemistry and Behavior, 27(1), 113-122. https://doi.org/10.1016/0091-3057(87)90485-0

Carrola, J., Rocha, C. M., Barros, A. S., Gil, A. M., Goodfellow, B. J., Carreira, I. M., ... Duarte, I. F. (2011). Metabolic Signatures of Lung Cancer in Biofluids: NMR-Based Metabonomics of Urine. Journal of Proteome Research, 10(1), 221-230. https://doi.org/10.1021/pr100899x

Carroll, M. E., \& Lac, S. T. (1997). Acquisition of IV amphetamine and cocaine selfadministration in rats as a function of dose. Psychopharmacology, 129(3), 206-214. https://doi.org/10.1007/s002130050182

Carroll, M. E., Lac, S. T., Asencio, M., \& Kragh, R. (1990a). Fluoxetine reduces intravenous cocaine self-administration in rats. Pharmacology Biochemistry and Behavior, 35(1), 237-244. https://doi.org/10.1016/0091-3057(90)90232-7

Carroll, M. E., Lac, S. T., Asencio, M., \& Kragh, R. (1990b). Intravenous cocaine selfadministration in rats is reduced by dietaryl-tryptophan. Psychopharmacology, 100(3), 293-300. https://doi.org/10.1007/BF02244596

Carter, L. P., \& Griffiths, R. R. (2009). Principles of laboratory assessment of drug abuse liability and implications for clinical development. Drug and Alcohol Dependence, 105 Suppl, S14-25. https://doi.org/10.1016/j.drugalcdep.2009.04.003

Cassidy, G., \& Ballard, C. G. (1994). Psychiatric sequelae of MDMA (ecstasy) and related drugs. Irish Journal of Psychological Medicine, 11(03), 132-133. https://doi.org/10.1017/S0790966700014841

Cervo, L., Carnovali, F., Stark, J. A., \& Mennini, T. (2003). Cocaine-Seeking Behavior in Response to Drug-Associated Stimuli in Rats: Involvement of D3 and D2 Dopamine Receptors. Neuropsychopharmacology, 28(6), 1150-1159. https://doi.org/10.1038/sj.npp.1300169

Chadwick, I. S., Curry, P. D., Linsley, A., Freemont, A. J., \& Doran, B. (1991). Ecstasy, 3-4 methylenedioxymethamphetamine (MDMA), a fatality associated with coagulopathy and hyperthermia. Journal of the Royal Society of Medicine, 84(6), 371. Retrieved from http://www.pubmedcentral.nih.gov/articlerender.fcgi?artid=1293290\&tool=pmcentrez\& rendertype $=$ abstract

Charara, A., \& Parent, A. (1998). Chemoarchitecture of the primate dorsal raphe nucleus. Journal of Chemical Neuroanatomy, 15(2), 111-127. https://doi.org/10.1016/S08910618(98)00036-2

Chefer, V. I., Thompson, A. C., Zapata, A., \& Shippenberg, T. S. (2009). Overview of Brain Microdialysis. Current Protocols in Neuroscience, 47(1), 7.1.1-7.1.28. https://doi.org/10.1002/0471142301.ns0701s47

Chen, J., Kelz, M. B., Hope, B. T., Nakabeppu, Y., \& Nestler, E. J. (1997). Chronic Fos-related antigens: stable variants of deltaFosB induced in brain by chronic treatments. The Journal of Neuroscience: The Official Journal of the Society for Neuroscience, 17(13), 49334941. https://doi.org/10.1523/JNEUROSCI.17-13-04933.1997

Childress, A. R., Ehrman, R. N., McLellan, A. T., \& O’Brien, C. P. (1988). Conditioned craving and arousal in cocaine addiction: a preliminary report. NIDA Res Monogr, 81, 7480.

Childress, A. R., Hole, A. V., Ehrman, R. N., Robbins, S. J., McLellan, A. T., \& O’Brien, C. P. (1993). Cue reactivity and cue reactivity interventions in drug dependence. NIDA Research Monograph, 137, 73.

Childress, A. R., Mozley, P. D., McElgin, W., Fitzgerald, J., Reivich, M., \& O’Brien, C. P. 
(1999). Limbic Activation During Cue-Induced Cocaine Craving. American Journal of Psychiatry, 156(1), 11-18. https://doi.org/10.1176/ajp.156.1.11

Cleary, L., \& Docherty, J. R. (2003). Actions of amphetamine derivatives and cathinone at the noradrenaline transporter. European Journal of Pharmacology, 476(1-2), 31-34. https://doi.org/10.1016/S0014-2999(03)02173-3

Clemens, K. J., Castino, M. R., Cornish, J. L., Goodchild, A. K., \& Holmes, N. M. (2014). Behavioral and Neural Substrates of Habit Formation in Rats Intravenously SelfAdministering Nicotine. Neuropsychopharmacology, 39(11), 2584-2593. https://doi.org/10.1038/npp.2014.111

Colby, C. R., Whisler, K., Steffen, C., Nestler, E. J., \& Self, D. W. (2003). Striatal Cell TypeSpecific Overexpression of $\Delta$ FosB Enhances Incentive for Cocaine. Journal of Neuroscience, 23(6), 2488-2493. https://doi.org/10.1523/JNEUROSCI.23-0602488.2003

Cole, J. C., \& Sumnall, H. R. (2003). The pre-clinical behavioural pharmacology of 3,4methylenedioxymethamphetamine (MDMA). Neuroscience \& Biobehavioral Reviews, 27(3), 199-217. https://doi.org/10.1016/S0149-7634(03)00031-9

Collins, R. J., Weeks, J. R., Cooper, M. M., Good, P. I., \& Russell, R. R. (1983). Prediction of abuse liability of drugs using IV self-administration by rats. Psychopharmacology, 82(12), 6-13. https://doi.org/10.1007/BF00426372

Colussi-Mas, J., \& Schenk, S. (2008). Acute and sensitized response to 3,4methylenedioxymethamphetamine in rats: different behavioral profiles reflected in different patterns of Fos expression. The European Journal of Neuroscience, 28(9), 18951910. https://doi.org/10.1111/j.1460-9568.2008.06467.x

Colussi-Mas, J., Wise, R. J., Howard, A., \& Schenk, S. (2010). Drug seeking in response to a priming injection of MDMA in rats: relationship to initial sensitivity to self-administered MDMA and dorsal striatal dopamine. Int J Neuropsychopharmacol, 13(10), 1315-1327. https://doi.org/10.1017/S1461145710000283

Commins, D. L., Vosmer, G., Virus, R. M., Woolverton, W. L., Schuster, C. R., \& Seiden, L. S. (1987). Biochemical and histological evidence that methylenedioxymethylamphetamine (MDMA) is toxic to neurons in the rat brain. $J$. Pharmacol. Exp. Ther., 241(1), 338-345. Retrieved from http://jpet.aspetjournals.org/content/241/1/338.short

Conversi, D., Bonito-Oliva, A., Orsini, C., Colelli, V., \& Cabib, S. (2007). DeltaFosB accumulation in ventro-medial caudate underlies the induction but not the expression of behavioral sensitization by both repeated amphetamine and stress. European Journal of Neuroscience, 27(1), 191-201. https://doi.org/10.1111/j.1460-9568.2007.06003.x

Corbit, L. H., Nie, H., \& Janak, P. H. (2012). Habitual Alcohol Seeking: Time Course and the Contribution of Subregions of the Dorsal Striatum. Biological Psychiatry, 72(5), 389395. https://doi.org/10.1016/J.BIOPSYCH.2012.02.024

Cornish, J. L., Hunt, G. E., Robins, L., \& McGregor, I. S. (2012). Regional c-Fos and FosB/ $\Delta$ FosB expression associated with chronic methamphetamine self-administration and methamphetamine-seeking behavior in rats. Neuroscience, 206, 100-114. https://doi.org/10.1016/J.NEUROSCIENCE.2012.01.004

Cornish, J. L., \& Kalivas, P. W. (2001). Repeated cocaine administration into the rat ventral tegmental area produces behavioral sensitization to a systemic cocaine challenge. Behavioural Brain Research, 126(1-2), 205-209. https://doi.org/10.1016/S01664328(01)00239-X

Corrigall, W. A., \& Coen, K. M. (1989). Nicotine maintains robust self-administration in rats on a limited-access schedule. Psychopharmacology, 99(4), 473-478. https://doi.org/10.1007/BF00589894 
Corrigall, W. A., \& Coen, K. M. (1991a). Cocaine self-administration is increased by both D1 and D2 dopamine antagonists. Pharmacology Biochemistry and Behavior, 39(3), 799802. https://doi.org/10.1016/0091-3057(91)90168-2

Corrigall, W. A., \& Coen, K. M. (1991b). Selective dopamine antagonists reduce nicotine selfadministration. Psychopharmacology, 104(2), 171-176. https://doi.org/10.1007/BF02244174

Costall, B., \& Naylor, R. J. (1979). Behavioural aspects of dopamine agonists and antagonists. In The neurobiology of dopamine (pp. 555-576). Academic Press London.

Costello, Z., \& Martin, H. G. (2018). A machine learning approach to predict metabolic pathway dynamics from time-series multiomics data. Npj Systems Biology and Applications, 4(1), 19. https://doi.org/10.1038/s41540-018-0054-3

Cottler, L. B., Leung, K. S., \& Abdallah, A. Ben. (2009). Test-re-test reliability of DSM-IV adopted criteria for 3,4-methylenedioxymethamphetamine (MDMA) abuse and dependence: a cross-national study. Addiction (Abingdon, England), 104(10), 1679-1690. https://doi.org/10.1111/j.1360-0443.2009.02649.x

Cottler, L. B., Womack, S. B., Compton, W. M., \& Ben-Abdallah, A. (2001). Ecstasy abuse and dependence among adolescents and young adults: applicability and reliability of DSM-IV criteria. Human Psychopharmacology: Clinical and Experimental, 16(8), 599606.

Creehan, K. M., Vandewater, S. A., \& Taffe, M. A. (2015). Intravenous self-administration of mephedrone, methylone and MDMA in female rats. Neuropharmacology, 92, 90-97. https://doi.org/10.1016/j.neuropharm.2015.01.003

Crespi, D., Mennini, T., \& Gobbi, M. (1997). Carrier-dependent and Ca(2+)-dependent 5-HT and dopamine release induced by (+)-amphetamine, 3,4methylendioxymethamphetamine, p-chloroamphetamine and (+)-fenfluramine. British Journal of Pharmacology, 121(8), 1735-1743. https://doi.org/10.1038/sj.bjp.0701325

Criswell, H. (1983). Intravenous self-administration of morphine by naive mice. Pharmacology Biochemistry and Behavior, 18(3), 467-470. https://doi.org/10.1016/00913057(83)90471-9

Crittenden, J. R., \& Graybiel, A. M. (2011). Basal Ganglia Disorders Associated with Imbalances in the Striatal Striosome and Matrix Compartments. Frontiers in Neuroanatomy, 5, 59. https://doi.org/10.3389/fnana.2011.00059

Crombag, H. S., Grimm, J. W., \& Shaham, Y. (2002). Effect of Dopamine Receptor Antagonists on Renewal of Cocaine Seeking by Reexposure to Drug-associated Contextual Cues. Neuropsychopharmacology, 27(6), 1006-1015. https://doi.org/10.1016/S0893-133X(02)00356-1

Cunningham, S. T., Finn, M., \& Kelley, A. E. (1997). Sensitization of the Locomotor Response to Psychostimulants after Repeated Opiate Exposure: Role of the Nucleus Accumbens. Neuropsychopharmacology, 16(2), 147-155. https://doi.org/10.1016/S0893133X(96)00166-2

Cunningham, S. T., \& Kelley, A. E. (1992). Evidence for opiate-dopamine cross-sensitization in nucleus accumbens: Studies of conditioned reward. Brain Research Bulletin, 29(5), 675-680. https://doi.org/10.1016/0361-9230(92)90137-M

Czoty, P. W., Ginsburg, B. C., \& Howell, L. L. (2002). Serotonergic Attenuation of the Reinforcing and Neurochemical Effects of Cocaine in Squirrel Monkeys. Journal of Pharmacology and Experimental Therapeutics, 300(3), 831-837. https://doi.org/10.1124/jpet.300.3.831

Dai, D., Gao, Y., Chen, J., Huang, Y., Zhang, Z., \& Xu, F. (2016). Time-resolved metabolomics analysis of individual differences during the early stage of lipopolysaccharide-treated rats. Scientific Reports, 6(1), 34136. https://doi.org/10.1038/srep34136 
Dalley, J. W., Cardinal, R. N., \& Robbins, T. W. (2004). Prefrontal executive and cognitive functions in rodents: neural and neurochemical substrates. Neuroscience \& Biobehavioral Reviews, 28(7), 771-784. https://doi.org/10.1016/J.NEUBIOREV.2004.09.006

Dalley, J. W., Everitt, B. J., \& Robbins, T. W. (2011). Impulsivity, Compulsivity, and TopDown Cognitive Control. Neuron, 69(4), 680-694. https://doi.org/10.1016/J.NEURON.2011.01.020

Daniela, E., Brennan, K. A., Gittings, D., Hely, L., \& Schenk, S. (2004). Effect of SCH 23390 on (+/-)-3,4-methylenedioxymethamphetamine hyperactivity and self-administration in rats. Pharmacology, Biochemistry, and Behavior, 77(4), 745-750. https://doi.org/10.1016/j.pbb.2004.01.008

Daniela, E., Gittings, D., \& Schenk, S. (2006). Conditioning Following Repeated Exposure to MDMA in Rats: Role in the Maintenance of MDMA Self-Administration. Behav Neurosci, 120(5), 1144.

Datta, U., van Staaden, M., \& Huber, R. (2018). Crayfish Self-Administer Amphetamine in a Spatially Contingent Task. Frontiers in Physiology, 9, 433. https://doi.org/10.3389/fphys.2018.00433

Davies, M. I. (1999). A review of microdialysis sampling for pharmacokinetic applications. Analytica Chimica Acta, 379(3), 227-249. https://doi.org/10.1016/S00032670(98)00633-3

Davies, M. I., Cooper, J. D., Desmond, S. S., Lunte, C. E., \& Lunte, S. M. (2000). Analytical considerations for microdialysis sampling. Advanced Drug Delivery Reviews, 45(2-3), 169-188. https://doi.org/10.1016/S0169-409X(00)00114-9

de la Torre, R., \& Farré, M. (2004). Neurotoxicity of MDMA (ecstasy): the limitations of scaling from animals to humans. Trends in Pharmacological Sciences, 25(10), 505-508. https://doi.org/10.1016/j.tips.2004.08.001

de la Torre, R., Farré, M., Ortuño, J., Mas, M., Brenneisen, R., Roset, P. N., ... Camí, J. (2000). Non-linear pharmacokinetics of MDMA ('ecstasy') in humans. British Journal of Clinical Pharmacology, 49(2), 104-109. https://doi.org/10.1046/j.1365-2125.2000.00121.x

de la Torre, R., Farré, M., Roset, P. N., Pizarro, N., Abanades, S., Segura, M., .. Camí, J. (2004). Human pharmacology of MDMA: pharmacokinetics, metabolism, and disposition. Therapeutic Drug Monitoring, 26(2), 137-144.

De Pauli, R. F., Coelhoso, C. C., Tesone-Coelho, C., Linardi, A., Mello, L. E., Silveira, D. X., \& Santos-Junior, J. G. (2014). Withdrawal induces distinct patterns of FosB/ $\Delta$ FosB expression in outbred Swiss mice classified as susceptible and resistant to ethanol-induced locomotor sensitization. Pharmacology Biochemistry and Behavior, 117, 70-78. https://doi.org/10.1016/J.PBB.2013.12.007

de Wit, H., \& Stewart, J. (1981). Reinstatement of cocaine-reinforced responding in the rat. Psychopharmacology, 75(2), 134-143. https://doi.org/10.1007/BF00432175

de Wit, H., \& Stewart, J. (1983). Drug reinstatement of heroin-reinforced responding in the rat. Psychopharmacology, 79(1), 29-31. https://doi.org/10.1007/BF00433012

Delfs, J. M., Schreiber, L., \& Kelley, A. E. (1990). Microinjection of cocaine into the nucleus accumbens elicits locomotor activation in the rat. J Neurosci, 10(1), 303-310.

Deneau, G., Yanagita, T., \& Seevers, M. H. (1969). Self-administration of psychoactive substances by the monkey. Psychopharmacologia, 16(1), 30-48. https://doi.org/10.1007/BF00405254

Deng, Y., Bu, Q., Hu, Z., Deng, P., Yan, G., Duan, J., ... Cen, X. (2012). 1H-nuclear magnetic resonance-based metabonomic analysis of brain in rhesus monkeys with morphine treatment and withdrawal intervention. Journal of Neuroscience Research, 90(11), 21542162. https://doi.org/10.1002/jnr.23109

Deroche-Gamonet, V., Belin, D., \& Piazza, P. V. (2004). Evidence for Addiction-like Behavior 
in the Rat. Science, 305(5686), 1014-1017. https://doi.org/10.1126/science.1099020

Deroche-Gamonet, V., Le Moal, M., \& Piazza, P. V. (1999). Cocaine self-administration increases the incentive motivational properties of the drug in rats. European Journal of Neuroscience, 11(8), 2731-2736. https://doi.org/10.1046/j.1460-9568.1999.00696.x

Dettmer, K., Aronov, P. A., \& Hammock, B. D. (2007). Mass spectrometry-based metabolomics. Mass Spectrometry Reviews, 26(1), 51-78. https://doi.org/10.1002/mas.20108

Di Benedetto, M., Bastías Candia, S. del C., D’Addario, C., Porticella, E. E., Cavina, C., Candeletti, S., \& Romualdi, P. (2011). Regulation of opioid gene expression in the rat brainstem by 3,4-methylenedioxymethamphetamine (MDMA): role of serotonin and involvement of CREB and ERK cascade. Naunyn-Schmiedeberg's Archives of Pharmacology, 383(2), 169-178. https://doi.org/10.1007/s00210-010-0587-5

Di Benedetto, M., D'Addario, C., Candeletti, S., \& Romualdi, P. (2006). Chronic and acute effects of 3,4-methylenedioxy-N-methylamphetamine ('ecstasy') administration on the dynorphinergic system in the rat brain. Neuroscience, 137(1), 187-196. https://doi.org/10.1016/J.NEUROSCIENCE.2005.09.015

Di Chiara, G., \& Imperato, A. (1988). Drugs abused by humans preferentially increase synaptic dopamine concentrations in the mesolimbic system of freely moving rats. Proceedings of the National Academy of Sciences, 85(14), 5274-5278. https://doi.org/10.1073/pnas.85.14.5274

Di Ciano, P. (2008). Facilitated Acquisition but Not Persistence of Responding for a CocainePaired Conditioned Reinforcer Following Sensitization with Cocaine. Neuropsychopharmacology, 33(6), 1426-1431. https://doi.org/10.1038/sj.npp.1301542

Di Ciano, P., Cardinal, R. N., Cowell, R. A., Little, S. J., \& Everitt, B. J. (2001). Differential involvement of NMDA, AMPA/kainate, and dopamine receptors in the nucleus accumbens core in the acquisition and performance of pavlovian approach behavior. The Journal of Neuroscience: The Official Journal of the Society for Neuroscience, 21(23), 9471-9477. Retrieved from http://www.ncbi.nlm.nih.gov/pubmed/11717381

Di Ciano, P., \& Everitt, B. J. (2004). Direct interactions between the basolateral amygdala and nucleus accumbens core underlie cocaine-seeking behavior by rats. The Journal of Neuroscience: The Official Journal of the Society for Neuroscience, 24(32), 7167-7173. https://doi.org/10.1523/JNEUROSCI.1581-04.2004

Di Ciano, P., Underwood, R. J., Hagan, J. J., \& Everitt, B. J. (2003). Attenuation of CueControlled Cocaine-Seeking by a Selective D3 Dopamine Receptor Antagonist SB277011-A. Neuropsychopharmacology, 28(2), 329-338. https://doi.org/10.1038/sj.npp.1300148

Di Matteo, V., Di Giovanni, G., Pierucci, M., \& Esposito, E. (2008). Serotonin control of central dopaminergic function: focus on in vivo microdialysis studies. Progress in Brain Research, 172, 7-44. https://doi.org/10.1016/S0079-6123(08)00902-3

Dianna, S. L., Smith, F. L., Smith, D. G., \& Lyness, W. H. (1986). Fluoxetine-induced attenuation of amphetamine self-administration in rats. Life Sciences, 39(15), 1383-1388.

Dickinson, A., Wood, N., \& Smith, J. W. (2002). Alcohol Seeking by Rats: Action or Habit? The Quarterly Journal of Experimental Psychology Section B, 55(4b), 331-348. https://doi.org/10.1080/0272499024400016

Do, J., \& Schenk, S. (2013). Self-administered MDMA produces dose- and time-dependent serotonin deficits in the rat brain. Addiction Biology, 18(3), 441-447. https://doi.org/10.1111/j.1369-1600.2011.00370.x

Downs, A. W., \& Eddy, N. B. (1932). The effect of repeated doses of cocaine on the rat. Journal of Pharmacology and Experimental Therapeutics, 46(2), 199-200.

Dworkin, S. I., Guerin, G., Co, C., Smith, J. E., \& Goeders, N. E. (1988). Effects of 5,7- 
dihydroxytryptamine lesions of the nucleus accumbens in rats responding on a concurrent schedule of food, water and intravenous morphine self-administration. NIDA Research Monograph, 81, 149-155. Retrieved from http://europepmc.org/abstract/med/3136354

Dykhuizen, R. S., Brunt, P. W., Atkinson, P., Simpson, J. G., \& Smith, C. C. (1995). Ecstasy induced hepatitis mimicking viral hepatitis. Gut, 36(6), 939-941. https://doi.org/10.1136/gut.36.6.939

Dyr, W., \& Kostowski, W. (1995). Evidence that the amygdala is involved in the inhibitory effects of 5-HT3 receptor antagonists on alcohol drinking in rats. Alcohol (Fayetteville, N.Y.), 12(4), 387-391. https://doi.org/10.1016/0741-8329(95)00023-k

Edwards, S., \& Koob, G. F. (2013). Escalation of drug self-administration as a hallmark of persistent addiction liability. Behavioural Pharmacology, 24(5-6), 356-362. https://doi.org/10.1097/FBP.0b013e3283644d15

Ehrman, R. N., Robbins, S. J., Childress, A. R., \& O’Brien, C. P. (1992). Conditioned responses to cocaine-related stimuli in cocaine abuse patients. Psychopharmacology, 107(4), 523529. https://doi.org/10.1007/BF02245266

Eiden, L. E., \& Weihe, E. (2011). VMAT2: a dynamic regulator of brain monoaminergic neuronal function interacting with drugs of abuse. Annals of the New York Academy of Sciences, 1216, 86-98. https://doi.org/10.1111/j.1749-6632.2010.05906.x

Entler, B. V, Cannon, J. T., \& Seid, M. A. (2016). Morphine addiction in ants: a new model for self-administration and neurochemical analysis. Journal of Experimental Biology, 219(18), 2865-2869.

Erb, S., Shaham, Y., \& Stewart, J. (1996). Stress reinstates cocaine-seeking behavior after prolonged extinction and a drug-free period. Psychopharmacology, 128(4), 408-412. https://doi.org/10.1007/s002130050150

Esteban, B., O’Shea, E., Camarero, J., Sanchez, V., Green, A. R., \& Colado, M. I. (2001). 3,4Methylenedioxymethamphetamine induces monoamine release, but not toxicity, when administered centrally at a concentration occurring following a peripherally injected neurotoxic dose. Psychopharmacology, 154(3), 251-260. https://doi.org/10.1007/s002130000645

Everitt, B. J., Belin, D., Economidou, D., Pelloux, Y., Dalley, J. W., \& Robbins, T. W. (2008). Neural mechanisms underlying the vulnerability to develop compulsive drug-seeking habits and addiction. Philosophical Transactions of the Royal Society B: Biological Sciences, 363(1507), 3125-3135. https://doi.org/10.1098/rstb.2008.0089

Everitt, B. J., \& Robbins, T. W. (2005). Neural systems of reinforcement for drug addiction: from actions to habits to compulsion. Nature Neuroscience, 8(11), 1481-1489. https://doi.org/10.1038/nn1579

Everitt, B. J., \& Robbins, T. W. (2016). Drug Addiction: Updating Actions to Habits to Compulsions Ten Years On. Annual Review of Psychology, 67(1), 23-50. https://doi.org/10.1146/annurev-psych-122414-033457

Fantegrossi, W. E., Woolverton, W. L., Kilbourn, M., Sherman, P., Yuan, J., Hatzidimitriou, G., ... Winger, G. (2004). Behavioral and neurochemical consequences of long-term intravenous self-administration of MDMA and its enantiomers by rhesus monkeys. Neuropsychopharmacology, 29(7), 1270-1281.

Feduccia, A. A., Kongovi, N., \& Duvauchelle, C. L. (2010). Heat increases MDMA-enhanced NAcc 5-HT and body temperature, but not MDMA self-administration. European Neuropsychopharmacology: The Journal of the European College of Neuropsychopharmacology, 20(12), 884-894. https://doi.org/10.1016/j.euroneuro.2010.08.009

Ferrario, C. R., \& Robinson, T. E. (2007). Amphetamine pretreatment accelerates the subsequent escalation of cocaine self-administration behavior. European 
Neuropsychopharmacology,

https://doi.org/10.1016/J.EURONEURO.2006.08.005

Fiehn, O. (2002). Metabolomics - the link between genotypes and phenotypes. In Functional Genomics (pp. 155-171). Dordrecht: Springer Netherlands. https://doi.org/10.1007/97894-010-0448-0_11

Fineschi, V., \& Masti, A. (1996). Fatal poisoning by MDMA (ecstasy) and MDEA: A case report. International Journal of Legal Medicine, 108(5), 272-275. https://doi.org/10.1007/BF01369826

Fischman, M. W., \& Schuster, C. R. (1981). Acute tolerance to cocaine in humans. NIDA Research Monograph, 34, 241-242. Retrieved from http://www.ncbi.nlm.nih.gov/pubmed/6783939

Fischman, M. W., Schuster, C. R., Javaid, J., Hatano, Y., \& Davis, J. (1985). Acute tolerance development to the cardiovascular and subjective effects of cocaine. Journal of Pharmacology and Experimental Therapeutics, 235(3).

Fitzgerald, J. L., \& Reid, J. J. (1990). Effects of methylenedioxymethamphetamine on the release of monoamines from rat brain slices. European Journal of Pharmacology, 191(2), 217-220. https://doi.org/10.1016/0014-2999(90)94150-V

Fleckenstein, A. E., Brown, J. M., Sandoval, V., Riddle, E. L., Hansen, J. P., Ugarte, Y. V, ... Hanson, G. R. (2002). D2 receptor-mediated regulation of vesicular dopamine uptake. In Catecholamine Research (pp. 39-42). Springer.

Fletcher, P. J., Korth, K. M., \& Chambers, J. W. (1999). Selective destruction of brain serotonin neurons by 5,7-dihydroxytryptamine increases responding for a conditioned reward. Psychopharmacology, 147(3), 291-299. https://doi.org/10.1007/s002130051170

Fletcher, P. J., Robinson, S. R., \& Slippoy, D. L. (2001). Pre-exposure to (+/-)3,4methylenedioxy-methamphetamine (MDMA) facilitates acquisition of intravenous cocaine self-administration in rats. Neuropsychopharmacology: Official Publication of the American College of Neuropsychopharmacology, 25(2), 195-203. https://doi.org/10.1016/S0893-133X(01)00222-6

Foltin, R. W., \& Fischman, M. W. (1991). Smoked and intravenous cocaine in humans: acute tolerance, cardiovascular and subjective effects. Journal of Pharmacology and Experimental Therapeutics, 257(1). Retrieved from http://jpet.aspetjournals.org/content/257/1/247.short

Food and Drug Administration [FDA]. (2010). Guidance for Industry: Assessment of Abuse Potential of Drugs. Retrieved from http://www.fda.gov/downloads/drugs/guidancecomplianceregulatoryinformation/guidan ces/ucm198650.pdf

Franken, I. H. A., Hendriks, V. M., Stam, C. J., \& Van den Brink, W. (2004). A role for dopamine in the processing of drug cues in heroin dependent patients. European Neuropsychopharmacology, 14(6), https://doi.org/10.1016/J.EURONEURO.2004.02.004

503-508.

Frankowska, A., Dudek, D., \& Siwek, M. (2016). $\Delta$ FosB protein and its role in the nucleus accumbens - an important factor in addiction, stress and response to natural rewards and psychoactive drugs. Neuropsychiatria $i$ Neuropsychologia, 2(2), 56-63. https://doi.org/10.5114/nan.2016.62250

Freedman, R. R., Johanson, C.-E., \& Tancer, M. E. (2005). Thermoregulatory effects of 3,4methylenedioxymethamphetamine (MDMA) in humans. Psychopharmacology, 183(2), 248-256. https://doi.org/10.1007/s00213-005-0149-6

Freezer, A., Salem, A., \& Irvine, R. (2005). Effects of 3,4-methylenedioxymethamphetamine (MDMA, 'Ecstasy') and para-methoxyamphetamine on striatal 5-HT when coadministered with moclobemide. Brain Research, 1041(1), 48-55. 
https://doi.org/10.1016/J.BRAINRES.2005.01.093

Fuchs, R. A., Evans, K. A., Parker, M. P., \& See, R. E. (2004). Differential Involvement of Orbitofrontal Cortex Subregions in Conditioned Cue-Induced and Cocaine-Primed Reinstatement of Cocaine Seeking in Rats. Journal of Neuroscience, 24(29), 6600-6610. https://doi.org/10.1523/JNEUROSCI.1924-04.2004

Fuchs, R. A., \& See, R. E. (2002). Basolateral amygdala inactivation abolishes conditioned stimulus- and heroin-induced reinstatement of extinguished heroin-seeking behavior in rats. Psychopharmacology, 160(4), 425-433. https://doi.org/10.1007/s00213-001-0997-7

Gabelica, V., \& Pauw, E. De. (2005). Internal energy and fragmentation of ions produced in electrospray sources. Mass Spectrometry Reviews, 24(4), 566-587. https://doi.org/10.1002/mas.20027

Gaiardi, M., Bartoletti, M., Bacchi, A., Gubellini, C., Costa, M., \& Babbini, M. (1991). Role of repeated exposure to morphine in determining its affective properties: place and taste conditioning studies in rats. Psychopharmacology, 103(2), 183-186. https://doi.org/10.1007/BF02244201

Gál, K., \& Gyertyán, I. (2006). Dopamine D3 as well as D2 receptor ligands attenuate the cueinduced cocaine-seeking in a relapse model in rats. Drug and Alcohol Dependence, 81(1), 63-70. https://doi.org/10.1016/J.DRUGALCDEP.2005.05.011

Galindo-Prieto, B., Eriksson, L., \& Trygg, J. (2014). Variable influence on projection (VIP) for orthogonal projections to latent structures (OPLS). Journal of Chemometrics, 28(8), 623-632. https://doi.org/10.1002/cem.2627

Galindo-Prieto, B., Eriksson, L., \& Trygg, J. (2015). Variable influence on projection (VIP) for OPLS models and its applicability in multivariate time series analysis. Chemometrics and Intelligent Laboratory Systems, 146, 297-304. https://doi.org/10.1016/J.CHEMOLAB.2015.05.001

Garavan, H., Pankiewicz, J., Bloom, A., Cho, J.-K., Sperry, L., Ross, T. J., ... Stein, E. A. (2000). Cue-Induced Cocaine Craving: Neuroanatomical Specificity for Drug Users and Drug Stimuli. American Journal of Psychiatry, 157(11), 1789-1798. https://doi.org/10.1176/appi.ajp.157.11.1789

García-Pardo, M. P., Miñarro, J., \& Aguilar, M. A. (2018). Role of AMPA glutamate receptors in the conditioned rewarding effects of MDMA in mice. Behavioural Brain Research, 347, 57-60. https://doi.org/10.1016/J.BBR.2018.03.010

García-Pardo, M. P., Miñarro, J., Llansola, M., Felipo, V., \& Aguilar, M. A. (2019). Role of NMDA and AMPA glutamatergic receptors in the effects of social defeat on the rewarding properties of MDMA in mice. European Journal of Neuroscience, 50(3), 2623-2634. https://doi.org/10.1111/ejn.14190

García-Repetto, R., Moreno, E., Soriano, T., Jurado, C., Giménez, M. P., \& Menéndez, M. (2003). Tissue concentrations of MDMA and its metabolite MDA in three fatal cases of overdose. Forensic Science International, 135(2), 110-114. https://doi.org/10.1016/S0379-0738(03)00179-8

Gardner, E. L. (2000). What We Have Learned about Addiction from Animal Models of Drug Self-Administration. The American Journal on Addictions, 9(4), 285-313. https://doi.org/10.1080/105504900750047355

Gardner, E. L. (2011). Addiction and brain reward and antireward pathways. Advances in Psychosomatic Medicine, 30, 22-60. https://doi.org/10.1159/000324065

Garrett, B. E., \& Holtzman, S. G. (1994). D1 and D2 dopamine receptor antagonists block caffeine-induced stimulation of locomotor activity in rats. Pharmacology Biochemistry and Behavior, 47(1), 89-94. https://doi.org/10.1016/0091-3057(94)90115-5

George, O., Mandyam, C. D., Wee, S., \& Koob, G. F. (2008). Extended Access to Cocaine Self-Administration Produces Long-Lasting Prefrontal Cortex-Dependent Working 
Memory Impairments. Neuropsychopharmacology, 33(10), 2474-2482. https://doi.org/10.1038/sj.npp.1301626

Gerber, G. J., \& Stretch, R. (1975). Drug-induced reinstatement of extinguished selfadministration behavior in monkeys. Pharmacology Biochemistry and Behavior, 3(6), 1055-1061. https://doi.org/10.1016/0091-3057(75)90016-7

Ghanbari, R., \& Sumner, S. J. (2018). Using Metabolomics to Investigate Biomarkers of Drug Addiction. Trends in Molecular Medicine, 24(2), 197-205. https://doi.org/10.1016/J.MOLMED.2017.12.005

Gilbert, J. G., Newman, A. H., Gardner, E. L., Ashby, C. R., Heidbreder, C. A., Pak, A. C., ... Xi, Z.-X. (2005). Acute administration of SB-277011A, NGB 2904, or BP 897 inhibits cocaine cue-induced reinstatement of drug-seeking behavior in rats: Role of dopamine D3 receptors. Synapse, 57(1), 17-28. https://doi.org/10.1002/syn.20152

Goeders, N. E., \& Smith, J. E. (1983). Cortical dopaminergic involvement in cocaine reinforcement. Science, 221(4612), 773-775. https://doi.org/10.1126/science.6879176

Gold, L. H., Hubner, C. B., \& Koob, G. F. (1989). A role for the mesolimbic dopamine system in the psychostimulant actions of MDMA. Psychopharmacology, 99(1), 40-47. https://doi.org/10.1007/BF00634450

Gold, L. H., Koob, G. F., \& Geyer, M. A. (1988). Stimulant and hallucinogenic behavioral profiles of 3,4- methylenedioxymethamphetamine and N-ethyl-3,4methylenedioxyamphetamine in rats. J. Pharmacol. Exp. Ther., 247(2), 547-555. Retrieved from http://jpet.aspetjournals.org/content/247/2/547.short

Gold, V., Hilton, J., \& Jefferson, E. G. (1954). A comparison of the mechanisms of the solvolytic hydrolysis reactions of acetic anhydride and benzoyl chloride. Journal of the Chemical Society (Resumed), 2756-2764.

Goldberg, S. R., Hoffmeister, F., Schlichting, U. U., \& Wuttke, W. (1971). A COMPARISON OF PENTOBARBITAL AND COCAINE SELF-ADMINISTRATION IN RHESUS MONKEYS: EFFECTS OF DOSE AND FIXED-RATIO PARAMETER. J. Pharmacol. Exp. Ther., 179(2), 277-283. Retrieved from http://jpet.aspetjournals.org/content/179/2/277.short

Goldberg, S. R., Kelleher, R. T., \& Morse, W. H. (1975). Second-order schedules of drug injection. Federation Proceedings, 34(9), 1771-1776. Retrieved from http://www.ncbi.nlm.nih.gov/pubmed/1149889

Goldberg, S. R., Woods, J. H., \& Schuster, C. R. (1971). Nalorphine-induced changes in morphine self-administration in rhesus monkeys. J. Pharmacol. Exp. Ther., 176(2), 464471. Retrieved from http://jpet.aspetjournals.org/content/176/2/464.short

Goldstein, R. Z., \& Volkow, N. D. (2011). Dysfunction of the prefrontal cortex in addiction: neuroimaging findings and clinical implications. Nature Reviews Neuroscience, 12(11), 652-669. https://doi.org/10.1038/nrn3119

Goodacre, R., Broadhurst, D. I., Smilde, A. K., Kristal, B. S., Baker, J. D., Beger, R., ... Wulfert, F. (2007). Proposed minimum reporting standards for data analysis in metabolomics. Metabolomics, 3(3), 231-241. https://doi.org/10.1007/s11306-007-0081-3

Górska, A. M., \& Gołembiowska, K. (2015). The Role of Adenosine A1 and A2A Receptors in the Caffeine Effect on MDMA-Induced DA and 5-HT Release in the Mouse Striatum. Neurotoxicity Research, 27(3), 229-245. https://doi.org/10.1007/s12640-014-9501-0

Götestam, K. G., \& Andersson, B. E. (1975). Self-administration of amphetamine analogues in rats. Pharmacology, Biochemistry, and Behavior, 3(2), 229-233. Retrieved from http://www.ncbi.nlm.nih.gov/pubmed/1170576

Gough, B., Imam, S. Z., Blough, B. E., Slikker, W., \& Ali, S. F. (2006). Comparative Effects of Substituted Amphetamines (PMA, MDMA, and METH) on Monoamines in Rat Caudate. Annals of the New York Academy of Sciences, 965(1), 410-420. 
https://doi.org/10.1111/j.1749-6632.2002.tb04182.x

Gould, R. W., Gage, H. D., Banks, M. L., Blaylock, B. L., Czoty, P. W., \& Nader, M. A. (2011). Differential effects of cocaine and MDMA self-administration on cortical serotonin transporter availability in monkeys. Neuropharmacology, 61(1-2), 245-251. https://doi.org/10.1016/j.neuropharm.2011.04.007

Green, A. R., Mechan, A. O., Elliott, J. M., O'Shea, E., \& Colado, M. I. (2003). The pharmacology and clinical pharmacology of 3,4-methylenedioxymethamphetamine (MDMA, “ecstasy”). Pharmacological Reviews, 55(3), 463-508. https://doi.org/10.1124/pr.55.3.3

Greenhouse, S. W., \& Geisser, S. (1959). On methods in the analysis of profile data. Psychometrika, 24(2), 95-112. https://doi.org/10.1007/BF02289823

Grimm, J. W., Hope, B. T., Wise, R. A., \& Shaham, Y. (2001). Incubation of cocaine craving after withdrawal. Nature, 412(6843), 141-142. https://doi.org/10.1038/35084134

Grimm, J. W., \& See, R. E. (2000). Dissociation of Primary and Secondary Reward-Relevant Limbic Nuclei in an Animal Model of Relapse. Neuropsychopharmacology, 22(5), 473479. https://doi.org/10.1016/S0893-133X(99)00157-8

Gromski, P. S., Muhamadali, H., Ellis, D. I., Xu, Y., Correa, E., Turner, M. L., \& Goodacre, R. (2015). A tutorial review: Metabolomics and partial least squares-discriminant analysis - a marriage of convenience or a shotgun wedding. Analytica Chimica Acta, 879, 10-23. https://doi.org/10.1016/J.ACA.2015.02.012

Grove, R. N., \& Schuster, C. R. (1974). Suppression of cocaine self-administration by extinction and punishment. Pharmacology Biochemistry and Behavior, 2(2), 199-208. https://doi.org/10.1016/0091-3057(74)90053-7

Grueter, B. A., Robison, A. J., Neve, R. L., Nestler, E. J., \& Malenka, R. C. (2013). $\Delta$ FosB differentially modulates nucleus accumbens direct and indirect pathway function. Proceedings of the National Academy of Sciences of the United States of America, 110(5), 1923-1928. https://doi.org/10.1073/pnas.1221742110

Gu, X. F., \& Azmitia, E. C. (1993). Integrative transporter-mediated release from cytoplasmic and vesicular 5-hydroxytryptamine stores in cultured neurons. European Journal of Pharmacology, 235(1), 51-57. https://doi.org/10.1016/0014-2999(93)90819-4

Gudelsky, G. A., \& Nash, J. F. (1996). Carrier-Mediated Release of Serotonin by 3,4Methylenedioxymethamphetamine: Implications for Serotonin-Dopamine Interactions. Journal of Neurochemistry, 66(1), 243-249. https://doi.org/10.1046/J.14714159.1996.66010243.X

Gudelsky, G. A., \& Nash, J. F. (2002). Carrier-Mediated Release of Serotonin by 3,4Methylenedioxymethamphetamine: Implications for Serotonin-Dopamine Interactions. Journal of Neurochemistry, 66(1), 243-249. https://doi.org/10.1046/j.14714159.1996.66010243.x

Gudelsky, G. A., \& Yamamoto, B. K. (2008). Actions of 3,4methylenedioxymethamphetamine (MDMA) on cerebral dopaminergic, serotonergic and cholinergic neurons. Pharmacology, Biochemistry, and Behavior, 90(2), 198-207. https://doi.org/10.1016/j.pbb.2007.10.003

Guo, H., Chen, J., Huang, Y., Zhang, W., Xu, F., \& Zhang, Z. (2016). A pseudo-kinetics approach for time-series metabolomics investigations: more reliable and sensitive biomarkers revealed in vincristine-induced paralytic ileus rats. RSC Advances, 6(59), 54471-54478. https://doi.org/10.1039/C6RA12641C

Hall, J., Parkinson, J. A., Connor, T. M., Dickinson, A., \& Everitt, B. J. (2001). Involvement of the central nucleus of the amygdala and nucleus accumbens core in mediating Pavlovian influences on instrumental behaviour. European Journal of Neuroscience, 13(10), 1984-1992. https://doi.org/10.1046/j.0953-816x.2001.01577.x 
Hall Jr, H. K. (1955). Mechanisms of Hydrolysis of Carbonyl Chlorides1. Journal of the American Chemical Society, 77(22), 5993-5996.

Halpern, J. H., Pope, H. G., Sherwood, A. R., Barry, S., Hudson, J. I., \& Yurgelun-Todd, D. (2004). Residual neuropsychological effects of illicit 3,4methylenedioxymethamphetamine (MDMA) in individuals with minimal exposure to other drugs. Drug and Alcohol Dependence, 75(2), 135-147. https://doi.org/10.1016/j.drugalcdep.2004.02.008

Hansen, S., Bergvall, A. H., \& Nyiredi, S. (1993). Interaction with pups enhances dopamine release in the ventral striatum of maternal rats: A microdialysis study. Pharmacology Biochemistry and Behavior, 45(3), 673-676. https://doi.org/10.1016/00913057(93)90523-V

Harmer, C. ., \& Phillips, G. D. (1998). Enhanced appetitive conditioning following repeated pretreatment with d-amphetamine. Behavioural Pharmacology, 9(4), 299-308. Retrieved from http://www.ncbi.nlm.nih.gov/pubmed/10065918

Harmer, C. ., \& Phillips, G. D. (1999). Enhanced dopamine efflux in the amygdala by a predictive, but not a non-predictive, stimulus: facilitation by prior repeated $\mathrm{d}$ amphetamine. Neuroscience, 90(1), 119-130. https://doi.org/10.1016/S03064522(98)00464-3

Harrigan, S. E., \& Downs, D. A. (1978). Self-administration of heroin, acetylmethadol, morphine, and methadone in rhesus monkeys. Life Sciences, 22(7), 619-623. https://doi.org/10.1016/0024-3205(78)90342-9

Hegadoren, K. M., Martin-Iverson, M. T., \& Baker, G. B. (1995). Comparative behavioural and neurochemical studies with a psychomotor stimulant, an hallucinogen and 3,4methylenedioxy analogues of amphetamine. Psychopharmacology, 118(3), 295-304. https://doi.org/10.1007/BF02245958

Hekmatpanah, C. R., \& Peroutka, S. J. (1990). 5-Hydroxytryptamine uptake blockers attenuate the 5-hydroxytryptamine-releasing effect of 3,4-methylenedioxymethamphetamine and related agents. European Journal of Pharmacology, 177(1-2), 95-98. https://doi.org/10.1016/0014-2999(90)90555-K

Henry, D. J., \& White, F. J. (1991). Repeated cocaine administration causes persistent enhancement of D1 dopamine receptor sensitivity within the rat nucleus accumbens. $J$. Pharmacol. Exp. Ther., 258(3), 882-890. Retrieved from http://jpet.aspetjournals.org/content/258/3/882.short

Heyser, C. J., Roberts, A. J., Schulteis, G., \& Koob, G. F. (1999). Central administration of an opiate antagonist decreases oral ethanol self-administration in rats. Alcoholism, Clinical and Experimental Research, 23(9), 1468-1476. Retrieved from http://www.ncbi.nlm.nih.gov/pubmed/10512312

Higgins, G. A., Wang, Y., Corrigall, W. A., \& Sellers, E. M. (1994). Influence of 5-HT3 receptor antagonists and the indirect 5-HT agonist, dexfenfluramine, on heroin selfadministration in rats. Psychopharmacology, 114(4), 611-619. https://doi.org/10.1007/BF02244992

Highgate, Q., \& Schenk, S. (2018). Comparison of the effects of abstinence on MDMA and cocaine self-administration in rats. Psychopharmacology, 235(11), 3233-3241. https://doi.org/10.1007/s00213-018-5026-1

Hochberg, Y. (1988). A sharper Bonferroni procedure for multiple tests of significance. Biometrika, 75(4), 800-802. https://doi.org/10.1093/biomet/75.4.800

Hoebel, B. G., Monaco, A. P., Hernandez, L., Aulisi, E. F., Stanley, B. G., \& Lenard, L. (1983). Self-injection of amphetamine directly into the brain. Psychopharmacology, 81(2), 158163. https://doi.org/10.1007/BF00429012

Hooks, M. S., Jones, G. H., Liem, B. J., \& Justice, J. B. (1992). Sensitization and individual 
differences to IP amphetamine, cocaine, or caffeine following repeated intracranial amphetamine infusions. Pharmacology Biochemistry and Behavior, 43(3), 815-823. https://doi.org/10.1016/0091-3057(92)90413-A

Hope, B. T., Nye, H. E., Kelz, M. B., Self, D. W., Iadarola, M. J., Nakabeppu, Y., .. Nestler,

E. J. (1994). Induction of a long-lasting AP-1 complex composed of altered Fos-like proteins in brain by chronic cocaine and other chronic treatments. Neuron, 13(5), 12351244. https://doi.org/10.1016/0896-6273(94)90061-2

Hopper, J. W., Su, Z., Looby, A. R., Ryan, E. T., Penetar, D. M., Palmer, C. M., \& Lukas, S. E. (2006). Incidence and patterns of polydrug use and craving for ecstasy in regular ecstasy users: an ecological momentary assessment study. Drug and Alcohol Dependence, 85(3), 221-235. https://doi.org/10.1016/j.drugalcdep.2006.04.012

Horger, B. A., Giles, M. K., \& Schenk, S. (1992). Preexposure to amphetamine and nicotine predisposes rats to self-administer a low dose of cocaine. Psychopharmacology, 107(23), 271-276. https://doi.org/10.1007/BF02245147

Horger, B. A., Shelton, K., \& Schenk, S. (1990). Preexposure sensitizes rats to the rewarding effects of cocaine. Pharmacology Biochemistry and Behavior, 37(4), 707-711. https://doi.org/10.1016/0091-3057(90)90552-S

Hori, K., Tanaka, J., \& Nomura, M. (1993). Effects of discrimination learning on the rat amygdala dopamine release: a microdialysis study. Brain Research, 621(2), 296-300. https://doi.org/10.1016/0006-8993(93)90119-8

Hornykiewicz, O. (1966). DOPAMINE (3-HYDROXYTYRAMINE) AND BRAIN FUNCTION. Pharmacol. Rev., 18(2), 925-964. Retrieved from http://pharmrev.aspetjournals.org/content/18/2/925.short

Howell, L. L., \& Byrd, L. D. (1991). Characterization of the effects of cocaine and GBR 12909, a dopamine uptake inhibitor, on behavior in the squirrel monkey. J. Pharmacol. Exp. Ther., 258(1), 178-185. Retrieved from http://jpet.aspetjournals.org/content/258/1/178.short

Howell, L. L., \& Byrd, L. D. (1995). Serotonergic modulation of the behavioral effects of cocaine in the squirrel monkey. Journal of Pharmacology and Experimental Therapeutics, 275(3), 1551-1559. Retrieved from http://jpet.aspetjournals.org/content/275/3/1551.abstract

Hubner, C. B., \& Edward Moreton, J. (1991). Effects of selective D1 and D2 dopamine antagonists on cocaine self-administration in the rat. Psychopharmacology, 105(2), 151156. https://doi.org/10.1007/BF02244301

Hudson, R. F., \& Wardill, J. E. (1950). 350. The mechanism of hydrolysis of acid chlorides. Part I. The effect of hydroxyl ions, temperature, and substituents on the rate of hydrolysis of benzoyl chloride. Journal of the Chemical Society (Resumed), 1729-1733.

Hurd, Y. L., McGrego, A., \& Pontén, M. (1997). In vivo Amygdala Dopamine Levels Modulate Cocaine Self-administration Behaviour in the Rat: D1 Dopamine Receptor Involvement. European Journal of Neuroscience, 9(12), 2541-2548. https://doi.org/10.1111/j.1460-9568.1997.tb01683.x

Hurd, Y. L., Weiss, F., Koob, G. F., And, N.-E., \& Ungerstedt, U. (1989). Cocaine reinforcement and extracellular dopamine overflow in rat nucleus accumbens: an in vivo microdialysis study. Brain Research, 498(1), 199-203. https://doi.org/10.1016/00068993(89)90422-8

Hyman, S. E., \& Malenka, R. C. (2001). Addiction and the brain: The neurobiology of compulsion and its persistence. Nature Reviews Neuroscience, 2(10), 695-703. https://doi.org/10.1038/35094560

Hysek, C. M., Simmler, L. D., Ineichen, M., Grouzmann, E., Hoener, M. C., Brenneisen, R., ... Liechti, M. E. (2011). The norepinephrine transporter inhibitor reboxetine reduces 
stimulant effects of MDMA ("ecstasy") in humans. Clinical Pharmacology and Therapeutics, 90(2), 246-255. https://doi.org/10.1038/clpt.2011.78

Hyytiä, P., \& Koob, G. F. (1995). GABAA receptor antagonism in the extended amygdala decreases ethanol self-administration in rats. European Journal of Pharmacology, 283(13), 151-159. https://doi.org/10.1016/0014-2999(95)00314-b

Insel, T. R., Battaglia, G., Johannessen, J., Marra, S., \& De Souza, E. B. (1989). 3,4Methylenedioxymethamphetamine ("ecstasy") selectively destroys brain serotonin terminals in rhesus monkeys. J. Pharmacol. Exp. Ther., 249(3), 713-720. Retrieved from http://jpet.aspetjournals.org/content/249/3/713.short

Inter-Agency Committee on Drugs. (2015). National Drug Policy 2015 to 2020. Wellington: Ministry of Health.

Iravani, M. M., Asari, D., Patel, J., Wieczorek, W. J., \& Kruk, Z. L. (2000). Direct effects of 3,4-methylenedioxymethamphetamine (MDMA) on serotonin or dopamine release and uptake in the caudate putamen, nucleus accumbens, substantia nigra pars reticulata, and the dorsal raphé nucleus slices. Synapse (New York, N.Y.), 36(4), 275-285. https://doi.org/10.1002/(SICI)1098-2396(20000615)36:4<275::AID-SYN4>3.0.CO;2-\#

Ito, R., Dalley, J. W., Howes, S. R., Robbins, T. W., \& Everitt, B. J. (2000). Dissociation in conditioned dopamine release in the nucleus accumbens core and shell in response to cocaine cues and during cocaine-seeking behavior in rats. The Journal of Neuroscience: The Official Journal of the Society for Neuroscience, 20(19), 7489-7495. Retrieved from http://www.ncbi.nlm.nih.gov/pubmed/11007908

Ito, R., Dalley, J. W., Robbins, T. W., \& Everitt, B. J. (2002). Dopamine release in the dorsal striatum during cocaine-seeking behavior under the control of a drug-associated cue. The Journal of Neuroscience: The Official Journal of the Society for Neuroscience, 22(14), 6247-6253. https://doi.org/20026606

Jentsch, J. D., Olausson, P., de la Garza, R., \& Taylor, J. R. (2002). Impairments of Reversal Learning and Response Perseveration after Repeated, Intermittent Cocaine Administrations to Monkeys. Neuropsychopharmacology, 26(2), 183-190. https://doi.org/10.1016/S0893-133X(01)00355-4

Jentsch, J. D., \& Taylor, J. R. (1999). Impulsivity resulting from frontostriatal dysfunction in drug abuse: implications for the control of behavior by reward-related stimuli. Psychopharmacology, 146(4), 373-390. https://doi.org/10.1007/PL00005483

Jog, M. S., Kubota, Y., Connolly, C. I., Hillegaart, V., \& Graybiel, A. M. (1999). Building neural representations of habits. Science, 286(5445), 1745-1749.

Johnson, M. P., Hoffman, A. J., \& Nichols, D. E. (1986). Effects of enantiomers of MDA, MDMA and related analogues on $[3 \mathrm{H}]$ serotonin and $[3 \mathrm{H}]$ dopamine release from superfused rat brain slices. European Journal of Pharmacology, 132(2-3), 269-276. https://doi.org/10.1016/0014-2999(86)90615-1

Jolliffe, I. (2011). Principle components analysis. Springer Berlin Heidelberg. Retrieved from https://link.springer.com/content/pdf/10.1007/978-3-642-04898-2_455.pdf

Jones, J. L., Day, J. J., Aragona, B. J., Wheeler, R. A., Wightman, R. M., \& Carelli, R. M. (2010). Basolateral Amygdala Modulates Terminal Dopamine Release in the Nucleus Accumbens and Conditioned Responding. Biological Psychiatry, 67(8), 737-744. https://doi.org/10.1016/J.BIOPSYCH.2009.11.006

Kalivas, P. W., \& Duffy, P. (1987). Sensitization to repeated morphine injection in the rat: possible involvement of A10 dopamine neurons. Journal of Pharmacology and Experimental Therapeutics, 241(1).

Kalivas, P. W., \& Duffy, P. (1990). Effect of acute and daily cocaine treatment on extracellular dopamine in the nucleus accumbens. Synapse (New York, N.Y.), 5(1), 48-58. https://doi.org/10.1002/syn.890050104 
Kalivas, P. W., \& Duffy, P. (1993a). Time course of extracellular dopamine and behavioral sensitization to cocaine. I. Dopamine axon terminals. The Journal of Neuroscience: The Official Journal of the Society for Neuroscience, 13(1), 266-275. https://doi.org/10.1523/JNEUROSCI.13-01-00266.1993

Kalivas, P. W., \& Duffy, P. (1993b). Time course of extracellular dopamine and behavioral sensitization to cocaine. II. Dopamine perikarya. The Journal of Neuroscience: The Official Journal of the Society for Neuroscience, 13(1), 276-284. https://doi.org/10.1523/JNEUROSCI.13-01-00276.1993

Kalivas, P. W., Duffy, P., \& White, S. R. (1998). MDMA elicits behavioral and neurochemical sensitization in rats. Neuropsychopharmacology: Official Publication of the American College of Neuropsychopharmacology, 18(6), 469-479. https://doi.org/10.1016/S0893133X(97)00195-4

Kalivas, P. W., \& Stewart, J. (1991). Dopamine transmission in the initiation and expression of drug- and stress-induced sensitization of motor activity. Brain Research Reviews, 16(3), 223-244. https://doi.org/10.1016/0165-0173(91)90007-U

Kalivas, P. W., \& Volkow, N. D. (2005). The Neural Basis of Addiction: A Pathology of Motivation and Choice. American Journal of Psychiatry, 162(8), 1403-1413. https://doi.org/10.1176/appi.ajp.162.8.1403

Kalivas, P. W., \& Weber, B. (1988). Amphetamine injection into the ventral mesencephalon sensitizes rats to peripheral amphetamine and cocaine. J. Pharmacol. Exp. Ther., 245(3), 1095-1102.

Retrieved

from http://jpet.aspetjournals.org/content/245/3/1095.abstract?ijkey=c6effe0175b373f3c975b 52b955062d1da3ee8d4\&keytype2=tf_ipsecsha

Kankaanpää, A., Meririnne, E., Lillsunde, P., \& Seppälä, T. (1998). The Acute Effects of Amphetamine Derivatives on Extracellular Serotonin and Dopamine Levels in Rat Nucleus Accumbens. Pharmacology Biochemistry and Behavior, 59(4), 1003-1009. https://doi.org/10.1016/S0091-3057(97)00527-3

Kantak, K. M., Black, Y., Valencia, E., Green-Jordan, K., \& Eichenbaum, H. B. (2002). Dissociable effects of lidocaine inactivation of the rostral and caudal basolateral amygdala on the maintenance and reinstatement of cocaine-seeking behavior in rats. The Journal of Neuroscience: The Official Journal of the Society for Neuroscience, 22(3), 1126-1136. Retrieved from http://www.ncbi.nlm.nih.gov/pubmed/11826141

Kaplan, G. B., Leite-Morris, K. A., Fan, W., Young, A. J., \& Guy, M. D. (2011). Opiate Sensitization Induces FosB $/ \Delta$ FosB Expression in Prefrontal Cortical, Striatal and

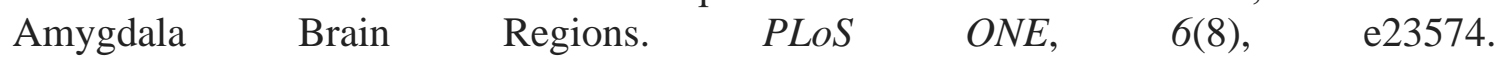
https://doi.org/10.1371/journal.pone.0023574

Kaste, K., Kivinummi, T., Piepponen, T. P., Kiianmaa, K., \& Ahtee, L. (2009). Differences in basal and morphine-induced $F o s B / \triangle F o s B$ and $p C R E B$ immunoreactivities in dopaminergic brain regions of alcohol-preferring AA and alcohol-avoiding ANA rats. Pharmacology Biochemistry and Behavior, 92(4), 655-662. https://doi.org/10.1016/J.PBB.2009.03.004

Katner, S., Magalong, J. G., \& Weiss, F. (1999). Reinstatement of Alcohol-Seeking Behavior by Drug-Associated Discriminative Stimuli after Prolonged Extinction in the Rat. Neuropsychopharmacology, 20(5), 471-479. https://doi.org/10.1016/S0893133X(98)00084-0

Kauer, J. A., \& Malenka, R. C. (2007). Synaptic plasticity and addiction. Nature Reviews Neuroscience, 8(11), 844-858. https://doi.org/10.1038/nrn2234

Kaufling, J., Waltisperger, E., Bourdy, R., Valera, A., Veinante, P., Freund-Mercier, M.-J., \& Barrot, M. (2010). Pharmacological recruitment of the GABAergic tail of the ventral tegmental area by acute drug exposure. British Journal of Pharmacology, 161(8), 1677- 
1691. https://doi.org/10.1111/j.1476-5381.2010.00984.x

Kehne, J. H., Ketteler, H. J., McCloskey, T. C., Sullivan, C. K., Dudley, M. W., \& Schmidt, C. J. (1996). Effects of the selective 5-HT2A receptor antagonist MDL 100,907 on MDMAinduced locomotor stimulation in rats. Neuropsychopharmacology : Official Publication of the American College of Neuropsychopharmacology, 15(2), 116-124. https://doi.org/10.1016/0893-133X(95)00160-F

Kelley, A. E., Gauthier, A. M., \& Lang, C. G. (1989). Amphetamine microinjections into distinct striatal subregions cause dissociable effects on motor and ingestive behavior. Behavioural Brain Research, 35(1), 27-39. https://doi.org/10.1016/S01664328(89)80005-1

Kelz, M. B., Chen, J., Carlezon, W. A., Whisler, K., Gilden, L., Beckmann, A. M., ... Nestler, E. J. (1999). Expression of the transcription factor $\Delta$ FosB in the brain controls sensitivity to cocaine. Nature, 401(6750), 272-276. https://doi.org/10.1038/45790

Khakoo, S. I., Coles, C. J., Armstrong, J. S., \& Barry, R. E. (1995). Hepatotoxicity and accelerated fibrosis following 3, 4-methylenedioxymetamphetamine (" ecstasy") usage. Journal of Clinical Gastroenterology, 20(3), 244-247.

Kim, H.-J., Kim, J. H., Noh, S., Hur, H. J., Sung, M. J., Hwang, J.-T., ... Yoon, S. H. (2011). Metabolomic Analysis of Livers and Serum from High-Fat Diet Induced Obese Mice. Journal of Proteome Research, 10(2), 722-731. https://doi.org/10.1021/pr100892r

Kim, H.-J., Lee, J. H., Yun, K., \& Kim, J.-H. (2017). Alterations in Striatal Circuits Underlying Addiction-Like Behaviors. Molecules and Cells, 40(6), 379-385. https://doi.org/10.14348/molcells.2017.0088

Kim, Y., Teylan, M. A., Baron, M., Sands, A., Nairn, A. C., \& Greengard, P. (2009). Methylphenidate-induced dendritic spine formation and DeltaFosB expression in nucleus accumbens. Proceedings of the National Academy of Sciences of the United States of America, 106(8), 2915-2920. https://doi.org/10.1073/pnas.0813179106

Kindlundh-Högberg, A. M., Blomqvist, A., Malki, R., \& Schiöth, H. B. (2008). Extensive neuroadaptive changes in cortical gene-transcript expressions of the glutamate system in response to repeated intermittent MDMA administration in adolescent rats. BMC Neuroscience, 9(1), 39. https://doi.org/10.1186/1471-2202-9-39

Kippin, T. E., Fuchs, R. A., \& See, R. E. (2006). Contributions of prolonged contingent and noncontingent cocaine exposure to enhanced reinstatement of cocaine seeking in rats. Psychopharmacology, 187(1), 60-67. https://doi.org/10.1007/s00213-006-0386-3

Kitamura, O., Wee, S., Specio, S. E., Koob, G. F., \& Pulvirenti, L. (2006). Escalation of methamphetamine self-administration in rats: a dose-effect function. Psychopharmacology, 186(1), 48-53. https://doi.org/10.1007/s00213-006-0353-z

Kleven, M. S., Woolverton, W. L., \& Seiden, L. S. (1989). Evidence that both intragastric and subcutaneous administration of methylenedioxymethylamphetamine (MDMA) produce serotonin neurotoxicity in rhesus monkeys. Brain Research, 488(1-2), 121-125. https://doi.org/10.1016/0006-8993(89)90700-2

Kolbrich, E. A., Goodwin, R. S., Gorelick, D. A., Hayes, R. J., Stein, E. A., \& Huestis, M. A. (2008). Plasma Pharmacokinetics of 3,4-Methylenedioxymethamphetamine After Controlled Oral Administration to Young Adults. Therapeutic Drug Monitoring, 30(3), 320-332. https://doi.org/10.1097/FTD.0b013e3181684fa0

Kolta, M. G., Shreve, P., \& Uretsky, N. J. (1989). Effect of pretreatment with amphetamine on the interaction between amphetamine and dopamine neurons in the nucleus accumbens. Neuropharmacology, 28(1), 9-14. https://doi.org/10.1016/0028-3908(89)90060-9

Koob, G. F. (2008). A Role for Brain Stress Systems in Addiction. Neuron, 59(1), 11-34. https://doi.org/10.1016/j.neuron.2008.06.012

Koob, G. F. (2013). Negative reinforcement in drug addiction: the darkness within. Current 
Opinion in Neurobiology, 23(4), 559-563. https://doi.org/10.1016/J.CONB.2013.03.011

Koob, G. F., Buck, C. L., Cohen, A., Edwards, S., Park, P. E., Schlosburg, J. E., ... George, O. (2014). Addiction as a stress surfeit disorder. Neuropharmacology, 76, 370-382. https://doi.org/10.1016/j.neuropharm.2013.05.024

Koob, G. F., Caine, S. B., Parsons, L. H., Markou, A., \& Weiss, F. (1997). Opponent Process Model and Psychostimulant Addiction. Pharmacology Biochemistry and Behavior, 57(3), 513-521. https://doi.org/https://doi.org/10.1016/S0091-3057(96)00438-8

Koob, G. F., Le, H. T., \& Creese, I. (1987). The D1 dopamine receptor antagonist SCH 23390 increases cocaine self-administration in the rat. Neuroscience Letters, 79(3), 315-320. https://doi.org/10.1016/0304-3940(87)90451-4

Koob, G. F., \& Le Moal, M. (2008a). Addiction and the Brain Antireward System. Annual $\begin{array}{llll}\text { Review of } & \text { Psychology, 59(1), }\end{array}$ https://doi.org/10.1146/annurev.psych.59.103006.093548

Koob, G. F., \& Le Moal, M. (2008b). Neurobiological mechanisms for opponent motivational processes in addiction. Philosophical Transactions of the Royal Society B: Biological Sciences, 363(1507), 3113-3123. https://doi.org/10.1098/rstb.2008.0094

Koob, G. F., \& Volkow, N. D. (2010). Neurocircuitry of Addiction. Neuropsychopharmacology, 35(1), 217-238. https://doi.org/10.1038/npp.2009.110

Koob, G. F., \& Volkow, N. D. (2016). Neurobiology of addiction: a neurocircuitry analysis. The Lancet. Psychiatry, 3(8), 760-773. https://doi.org/10.1016/S2215-0366(16)00104-8

Kramer, J. C., Fischman, V. S., \& Littlefield, D. C. (1967). Amphetamine Abuse: Pattern and Effects of High Doses Taken Intravenously. JAMA, 201(5), 305. https://doi.org/10.1001/jama.1967.03130050039011

Krasnova, I. N., Chiflikyan, M., Justinova, Z., McCoy, M. T., Ladenheim, B., Jayanthi, S., ... Cadet, J. L. (2013). CREB phosphorylation regulates striatal transcriptional responses in the self-administration model of methamphetamine addiction in the rat. Neurobiology of Disease, 58, 132-143. https://doi.org/10.1016/J.NBD.2013.05.009

Kuhl, C., Tautenhahn, R., Böttcher, C., Larson, T. R., \& Neumann, S. (2012). CAMERA: An Integrated Strategy for Compound Spectra Extraction and Annotation of Liquid Chromatography/Mass Spectrometry Data Sets. Analytical Chemistry, 84(1), 283-289. https://doi.org/10.1021/ac202450g

Kurling, S., Kankaanpää, A., \& Seppälä, T. (2008). Sub-chronic nandrolone treatment modifies neurochemical and behavioral effects of amphetamine and 3,4methylenedioxymethamphetamine (MDMA) in rats. Behavioural Brain Research, 189(1), 191-201. https://doi.org/10.1016/j.bbr.2007.12.021

Lamb, R. J., \& Griffiths, R. R. (1990). Self-administration in baboons and the discriminative stimulus effects in rats of bupropion, nomifensine, diclofensine and imipramine. Psychopharmacology, 102(2), 183-190. https://doi.org/10.1007/BF02245920

Larson, E. B., Akkentli, F., Edwards, S., Graham, D. L., Simmons, D. L., Alibhai, I. N., ... Self, D. W. (2010). Striatal regulation of $\triangle$ FosB, FosB, and cFos during cocaine selfadministration and withdrawal. Journal of Neurochemistry, 115(1), 112-122. https://doi.org/10.1111/j.1471-4159.2010.06907.x

Leccese, A. P., \& Lyness, W. H. (1984). The effects of putative 5-hydroxytryptamine receptor active agents ond-amphetamine self-administration in controls and rats with 5,7dihydroxytryptamine median forebrain bundle lesion. Brain Research, 303(1), 153-162. https://doi.org/10.1016/0006-8993(84)90223-3

Leonardi, E. T., \& Azmitia, E. C. (1994). MDMA (ecstasy) inhibition of MAO type A and type B: comparisons with fenfluramine and fluoxetine (Prozac). Neuropsychopharmacology: Official Publication of the American College of Neuropsychopharmacology, 10(4), 231238. https://doi.org/10.1038/npp.1994.26 
Lett, B. T. (1989). Repeated exposures intensify rather than diminish the rewarding effects of amphetamine, morphine, and cocaine. Psychopharmacology, 98(3), 357-362. https://doi.org/10.1007/BF00451687

Leyton, M., Casey, K. F., Delaney, J. S., Kolivakis, T., \& Benkelfat, C. (2005). Cocaine craving, euphoria, and self-administration: A preliminary study of the effect of catecholamine precursor depletion. Behavioral Neuroscience, 119(6), 1619-1627. https://doi.org/10.1037/0735-7044.119.6.1619

Li, H., Chen, B., Shao, X., Hu, Z., Deng, Y., Zhu, R., ... Cen, X. (2014). 1H-Nuclear magnetic resonance-based metabolomic analysis of brain in mice with nicotine treatment. BMC Neuroscience, 15(1), 32. https://doi.org/10.1186/1471-2202-15-32

Li, P., Wu, P., Xin, X., Fan, Y.-L., Wang, G.-B., Wang, F., ... Lu, L. (2015). Incubation of alcohol craving during abstinence in patients with alcohol dependence. Addiction Biology, 20(3), 513-522. https://doi.org/10.1111/adb.12140

Li, X., Caprioli, D., \& Marchant, N. J. (2015). Recent updates on incubation of drug craving: a mini-review. Addiction Biology, 20(5), 872-876. https://doi.org/10.1111/adb.12205

Li, Yilin, Acerbo, M. J., \& Robinson, T. E. (2004). The induction of behavioural sensitization is associated with cocaine-induced structural plasticity in the core (but not shell) of the nucleus accumbens. European Journal of Neuroscience, 20(6), 1647-1654. https://doi.org/10.1111/j.1460-9568.2004.03612.x

Li, Yilin, Kolb, B., \& Robinson, T. E. (2003). The Location of Persistent AmphetamineInduced Changes in the Density of Dendritic Spines on Medium Spiny Neurons in the Nucleus Accumbens and Caudate-Putamen. Neuropsychopharmacology, 28(6), 10821085. https://doi.org/10.1038/sj.npp.1300115

Li, Yong, Hu, X.-T., Berney, T. G., Vartanian, A. J., Stine, C. D., Wolf, M. E., \& White, F. J. (1999). Both glutamate receptor antagonists and prefrontal cortex lesions prevent induction of cocaine sensitization and associated neuroadaptations. Synapse, 34(3), 169180. $\quad$ https://doi.org/10.1002/(SICI)1098-2396(19991201)34:3<169::AIDSYN1>3.0.CO;2-C

Li, Z., Lu, Y., Guo, Y., Cao, H., Wang, Q., \& Shui, W. (2018). Comprehensive evaluation of untargeted metabolomics data processing software in feature detection, quantification and discriminating marker selection. Analytica Chimica Acta, 1029, 50-57. https://doi.org/10.1016/J.ACA.2018.05.001

Livera, A. M. De, Sysi-Aho, M., Jacob, L., Gagnon-Bartsch, J. A., Castillo, S., Simpson, J. A., \& Speed, T. P. (2015). Statistical Methods for Handling Unwanted Variation in Metabolomics Data. Analytical Chemistry, 87(7), 3606-3615. https://doi.org/10.1021/ac502439y

Loos, M., Gerber, C., Corona, F., Hollender, J., \& Singer, H. (2015). Accelerated Isotope Fine Structure Calculation Using Pruned Transition Trees. Analytical Chemistry, 87(11), 5738-5744. https://doi.org/10.1021/acs.analchem.5b00941

Lorrain, D. S., Arnold, G. M., \& Vezina, P. (2000). Previous exposure to amphetamine increases incentive to obtain the drug: long-lasting effects revealed by the progressive ratio schedule. Behavioural Brain Research, 107(1-2), 9-19. https://doi.org/10.1016/S0166-4328(99)00109-6

Louilot, A., Simon, H., Taghzouti, K., \& Le Moal, M. (1985). Modulation of dopaminergic activity in the nucleus accumbens following facilitation or blockade of the dopaminergic transmission in the amygdala: a study by in vivo differential pulse voltammetry. Brain Research, 346(1), 141-145. https://doi.org/10.1016/0006-8993(85)91104-7

Lynch, W. J. (2006). Sex differences in vulnerability to drug self-administration. Experimental and Clinical Psychopharmacology, 14(1), 34-41. https://doi.org/10.1037/10641297.14.1.34 
Lyness, W. H. (1983). Effect of L-tryptophan pretreatment on d-amphetamine self administration. Substance \& Alcohol Actions/Misuse.

Lyness, W. H., Friedle, N. M., \& Moore, K. E. (1979). Destruction of dopaminergic nerve terminals in nucleus accumbens: Effect on d-amphetamine self-administration. Pharmacology Biochemistry and Behavior, 11(5), 553-556. https://doi.org/10.1016/0091-3057(79)90040-6

Lyness, W. H., Friedle, N. M., \& Moore, K. E. (1980). Increased self-administration of damphetamine after destruction of 5-hydroxytryptaminergic neurons. Pharmacology Biochemistry and Behavior, 12(6), 937-941. https://doi.org/10.1016/00913057(80)90456-6

Lyness, W. H., \& Smith, J. E. (1992). Influence of dopaminergic and serotonergic neurons on intravenous ethanol self-administration in the rat. Pharmacology, Biochemistry, and Behavior, 42(1), 187-192. Retrieved from http://www.ncbi.nlm.nih.gov/pubmed/1388276

Maan, A., Highgate, Q., \& Schenk, S. (2020). A comparison of acquisition, maintenance, and extinction of MDMA and methamphetamine self-administartion in rats. Manuscript in Preperation.

Madsen, H. B., Brown, R. M., \& Lawrence, A. J. (2012). Neuroplasticity in addiction: cellular and transcriptional perspectives. Frontiers in Molecular Neuroscience, 5, 99. https://doi.org/10.3389/fnmol.2012.00099

Mantsch, J., Yuferov, V., Mathieu-Kia, A.-M., Ho, A., \& Kreek, M. (2004). Effects of extended access to high versus low cocaine doses on self-administration, cocaine-induced reinstatement and brain mRNA levels in rats. Psychopharmacology, 175(1), 26-36. https://doi.org/10.1007/s00213-004-1778-x

Marttila, K., Raattamaa, H., \& Ahtee, L. (2006). Effects of chronic nicotine administration and its withdrawal on striatal $\mathrm{FosB} / \triangle \mathrm{FosB}$ and $\mathrm{c}-\mathrm{Fos}$ expression in rats and mice. Neuropharmacology, 51(1), 44-51. https://doi.org/10.1016/J.NEUROPHARM.2006.02.014

Mas, M., Farré, M., de la Torre, R., Roset, P. N., Ortuño, J., Segura, J., \& Camí, J. (1999). Cardiovascular and neuroendocrine effects and pharmacokinetics of 3, 4methylenedioxymethamphetamine in humans. The Journal of Pharmacology and Experimental Therapeutics, 290(1), 136-145. Retrieved from http://www.ncbi.nlm.nih.gov/pubmed/10381769

Mas, Manuel, Fumero, B., \& González-Mora, J. (1995). Voltammetric and microdialysis monitoring of brain monoamine neurotransmitter release during sociosexual interactions. Behavioural Brain Research, 71(1-2), 69-IN5. https://doi.org/10.1016/01664328(95)00043-7

Mattox, A. J., \& Carroll, M. E. (1996). Smoked heroin self-administration in rhesus monkeys. Psychopharmacology, 125(3), 195-201. https://doi.org/10.1007/BF02247328

Maurer, H. H., Bickeboeller-Friedrich, J., Kraemer, T., \& Peters, F. T. (2000). Toxicokinetics and analytical toxicology of amphetamine-derived designer drugs ('Ecstasy'). Toxicology Letters, 112-113, 133-142. https://doi.org/10.1016/S0378-4274(99)00207-6

Mayerhofer, A., Kovar, K.-A., \& Schmidt, W. J. (2001). Changes in serotonin, dopamine and noradrenaline levels in striatum and nucleus accumbens after repeated administration of the abused drug MDMA in rats. Neuroscience Letters, 308(2), 99-102. https://doi.org/10.1016/S0304-3940(01)01992-9

Maze, I., Covington, H. E., Dietz, D. M., LaPlant, Q., Renthal, W., Russo, S. J., ... Nestler, E. J. (2010). Essential Role of the Histone Methyltransferase G9a in Cocaine-Induced Plasticity. Science, 327(5962), 213-216. https://doi.org/10.1126/science.1179438

McBride, W. J., Murphy, J. M., \& Ikemoto, S. (1999). Localization of brain reinforcement 
mechanisms: intracranial self-administration and intracranial place-conditioning studies. Behavioural Brain Research, 101(2), 129-152. https://doi.org/10.1016/S01664328(99)00022-4

McCann, U. D., Ridenour, A., Shaham, Y., \& Ricaurte, G. A. (1994). Serotonin neurotoxicity after (+/-)3,4-methylenedioxymethamphetamine (MDMA; "Ecstasy"): a controlled study in humans. Neuropsychopharmacology: Official Publication of the American College of Neuropsychopharmacology, 10(2), 129-138. https://doi.org/10.1038/npp.1994.15

McCann, U. D., Szabo, Z., Scheffel, U., Dannals, R., \& Ricaurte, G. A. (1998). Positron emission tomographic evidence of toxic effect of MDMA ("Ecstasy") on brain serotonin neurons in human beings. The Lancet, 352(9138), 1433-1437. https://doi.org/10.1016/S0140-6736(98)04329-3

McClung, C. A., \& Nestler, E. J. (2003). Regulation of gene expression and cocaine reward by CREB and $\triangle$ FosB. Nature Neuroscience, 6(11), 1208-1215. https://doi.org/10.1038/nn1143

McClung, C. A., Ulery, P. G., Perrotti, L. I., Zachariou, V., Berton, O., \& Nestler, E. J. (2004). $\triangle$ FosB: a molecular switch for long-term adaptation in the brain. Molecular Brain Research, 132(2), 146-154. https://doi.org/10.1016/J.MOLBRAINRES.2004.05.014

McDaid, J., Graham, M. P., \& Napier, T. C. (2006). Methamphetamine-induced sensitization differentially alters pCREB and DeltaFosB throughout the limbic circuit of the mammalian brain. Molecular Pharmacology, 70(6), 2064-2074. https://doi.org/10.1124/mol.106.023051

McFadden Consultancy. (2016). Research Report: The New Zealand Drug Harm Index 2016 (2nd edn). Wellington: Ministry of Health.

McFarland, K., Davidge, S. B., Lapish, C. C., \& Kalivas, P. W. (2004). Limbic and motor circuitry underlying footshock-induced reinstatement of cocaine-seeking behavior. The Journal of Neuroscience: The Official Journal of the Society for Neuroscience, 24(7), 1551-1560. https://doi.org/10.1523/JNEUROSCI.4177-03.2004

McFarland, K., \& Ettenberg, A. (1997). Reinstatement of drug-seeking behavior produced by heroin-predictive environmental stimuli. Psychopharmacology, 131(1), 86-92. https://doi.org/10.1007/s002130050269

McFarland, K., \& Kalivas, P. W. (2001). The circuitry mediating cocaine-induced reinstatement of drug-seeking behavior. The Journal of Neuroscience: The Official Journal of the Society for Neuroscience, 21(21), 8655-8663. https://doi.org/10.1523/JNEUROSCI.21-21-08655.2001

McFarland, K., Lapish, C. C., \& Kalivas, P. W. (2003). Prefrontal glutamate release into the core of the nucleus accumbens mediates cocaine-induced reinstatement of drug-seeking behavior. The Journal of Neuroscience: The Official Journal of the Society for Neuroscience, 23(8), 3531-3537. https://doi.org/10.1523/JNEUROSCI.23-0803531.2003

McGregor, A., Lacosta, S., \& Roberts, D. C. S. (1993). L-tryptophan decreases the breaking point under a progressive ratio schedule of intravenous cocaine reinforcement in the rat. Pharmacology Biochemistry and Behavior, 44(3), 651-655.

McGregor, A., \& Roberts, D. C. S. (1993). Dopaminergic antagonism within the nucleus accumbens or the amygdala produces differential effects on intravenous cocaine selfadministration under fixed and progressive ratio schedules of reinforcement. Brain Research, 624(1-2), 245-252. https://doi.org/10.1016/0006-8993(93)90084-z

McGuire, P. K., Cope, H., \& Fahy, T. A. (1994). Diversity of psychopathology associated with use of 3, 4-methylenedioxymethamphetamine ('Ecstasy'). The British Journal of Psychiatry, 165(3), 391-395.

McKetin, R., Copeland, J., Norberg, M. M., Bruno, R., Hides, L., \& Khawar, L. (2014). The 
effect of the ecstasy "come-down" on the diagnosis of ecstasy dependence. Drug and Alcohol Dependence, 139, 26-32. https://doi.org/10.1016/j.drugalcdep.2014.02.697

McLaughlin, J., \& See, R. E. (2003). Selective inactivation of the dorsomedial prefrontal cortex and the basolateral amygdala attenuates conditioned-cued reinstatement of extinguished cocaine-seeking behavior in rats. Psychopharmacology, 168(1-2), 57-65. https://doi.org/10.1007/s00213-002-1196-x

Mead, A. N., Crombag, H. S., \& Rocha, B. A. (2004). Sensitization of Psychomotor Stimulation and Conditioned Reward in Mice: Differential Modulation by Contextual Learning. Neuropsychopharmacology, 29(2), 249-258. https://doi.org/10.1038/sj.npp.1300294

Meil, W. M., \& See, R. E. (1996). Conditioned cued recovery of responding following prolonged withdrawal from self-administered cocaine in rats: an animal model of relapse. Behavioural Pharmacology.

Meil, W. M., \& See, R. E. (1997). Lesions of the basolateral amygdala abolish the ability of drug associated cues to reinstate responding during withdrawal from self-administered cocaine. Behavioural Brain Research, 87(2), 139-148. https://doi.org/10.1016/S01664328(96)02270-X

Meinhardt, M. W., Sévin, D. C., Klee, M. L., Dieter, S., Sauer, U., \& Sommer, W. H. (2015). The Neurometabolic Fingerprint of Excessive Alcohol Drinking. Neuropsychopharmacology, 40(5), 1259-1268. https://doi.org/10.1038/npp.2014.312

Mendrek, A., Blaha, C. D., \& Phillips, A. G. (1998). Pre-exposure of rats to amphetamine sensitizes self-administration of this drug under a progressive ratio schedule. Psychopharmacology, 135(4), 416-422. https://doi.org/10.1007/s002130050530

Mews, P., Walker, D. M., \& Nestler, E. J. (2019). Epigenetic Priming in Drug Addiction. Cold Spring Harbor Symposia on Quantitative Biology, 037663. https://doi.org/10.1101/sqb.2018.83.037663

Miles, F. J., Everitt, B. J., \& Dickinson, A. (2003). Oral cocaine seeking by rats: Action or habit? Behavioral Neuroscience, 117(5), 927-938. https://doi.org/10.1037/07357044.117.5.927

Miller, G. M. (2011). The emerging role of trace amine-associated receptor 1 in the functional regulation of monoamine transporters and dopaminergic activity. Journal of Neurochemistry, 116(2), 164-176. https://doi.org/10.1111/j.1471-4159.2010.07109.x

Ministry of Health. (2013). Health Loss in New Zealand: A report from the New Zealand Burden of Diseases, Injuries and Rick Factors Study, 2006-2016. Wellington: Ministry of Health.

Ministry of Health. (2016). Health loss in New Zealand 1990-2013: A report from the New Zealand Burden of Diseases, Injuries, and Risk Factors Study. Wellington: Ministry of Health.

Möller, C., Wiklund, L., Sommer, W. H., Thorsell, A., \& Heilig, M. (1997). Decreased experimental anxiety and voluntary ethanol consumption in rats following central but not basolateral amygdala lesions. Brain Research, 760(1-2), 94-101. https://doi.org/10.1016/s0006-8993(97)00308-9

Moosavi, S. M., \& Ghassabian, S. (2018). Linearity of Calibration Curves for Analytical Methods: A Review of Criteria for Assessment of Method Reliability. Calibration and Validation of Analytical Methods: A Sampling of Current Approaches. BoD-Books on Demand.

Moratalla, R., Elibol, B., Vallejo, M., \& Graybiel, A. M. (1996). Network-Level Changes in Expression of Inducible Fos-Jun Proteins in the Striatum during Chronic Cocaine Treatment and Withdrawal. Neuron, 17(1), 147-156. https://doi.org/10.1016/S08966273(00)80288-3 
Morgan, A. E., Horan, B., Dewey, S. L., \& Ashby, C. R. (1997). Repeated administration of 3,4-methylenedioxymethamphetamine augments cocaine's action on dopamine in the nucleus accumbens: a microdialysis study. Eur $J$ Pharmacol, 331(1), R1-3. https://doi.org/10.1016/S0014-2999(97)01035-2

Moyano, S., Del Río, J., \& Frechilla, D. (2005). Acute and chronic effects of MDMA on molecular mechanisms implicated in memory formation in rat hippocampus: Surface expression of CaMKII and NMDA receptor subunits. Pharmacology Biochemistry and Behavior, 82(1), 190-199. https://doi.org/10.1016/J.PBB.2005.07.020

Moyano, S., Frechilla, D., \& Del Río, J. (2004). NMDA receptor subunit and CaMKII changes in rat hippocampus induced by acute MDMA treatment: a mechanism for learning impairment. Psychopharmacology, 173(3-4), 337-345. https://doi.org/10.1007/s00213004-1816-8

Muller, D. L., Unterwald, E. M., \& Fujimoto, J. M. (2005). D1 dopamine receptors modulate deltaFosB induction in rat striatum after intermittent morphine administration. The Journal of Pharmacology and Experimental Therapeutics, 314(1), 148-154. https://doi.org/10.1124/jpet.105.083410

Munzar, P., Baumann, M. H., Shoaib, M., \& Goldberg, S. R. (1999). Effects of dopamine and serotonin-releasing agents on methamphetamine discrimination and self-administration in rats. Psychopharmacology, 141(3), 287-296. https://doi.org/10.1007/s002130050836

Murray, J. E., Belin, D., \& Everitt, B. J. (2012). Double Dissociation of the Dorsomedial and Dorsolateral Striatal Control Over the Acquisition and Performance of Cocaine Seeking. Neuropsychopharmacology, 37(11), 2456-2466. https://doi.org/10.1038/npp.2012.104

Mussap, M., Loddo, C., Fanni, C., \& Fanos, V. (2020). Metabolomics in pharmacology - a delve into the novel field of pharmacometabolomics. Expert Review of Clinical Pharmacology, 1-20. https://doi.org/10.1080/17512433.2020.1713750

Myers, O. D., Sumner, S. J., Li, S., Barnes, S., \& Du, X. (2017a). Detailed Investigation and Comparison of the XCMS and MZmine 2 Chromatogram Construction and Chromatographic Peak Detection Methods for Preprocessing Mass Spectrometry Metabolomics Data. Analytical Chemistry, 89(17), 8689-8695. https://doi.org/10.1021/acs.analchem.7b01069

Myers, O. D., Sumner, S. J., Li, S., Barnes, S., \& Du, X. (2017b). One Step Forward for Reducing False Positive and False Negative Compound Identifications from Mass Spectrometry Metabolomics Data: New Algorithms for Constructing Extracted Ion Chromatograms and Detecting Chromatographic Peaks. Analytical Chemistry, 89(17), 8696-8703. https://doi.org/10.1021/acs.analchem.7b00947

Nader, M. A., \& Mach, R. H. (1996). Self-administration of the dopamine D3 agonist 7-OHDPAT in rhesus monkeys is modified by prior cocaine exposure. Psychopharmacology, 125(1), 13-22. https://doi.org/10.1007/BF02247388

Nair, S. G., \& Gudelsky, G. A. (2004). Protein kinase C inhibition differentially affects 3,4methylenedioxymethamphetamine-induced dopamine release in the striatum and prefrontal cortex of the rat. Brain Research, 1013(2), 168-173. https://doi.org/10.1016/J.BRAINRES.2004.04.007

Nanopoulos, D., Belin, M. F., Maitre, M., Vincendon, G., \& Pujol, J. F. (1982). Immunocytochemical evidence for the existence of GABAergic neurons in the nucleus raphe dorsalis. possible existence of neurons containing serotonin and GABA. Brain Research, 232(2), 375-389. https://doi.org/10.1016/0006-8993(82)90281-5

Nash, J. F. (1990). Ketanserin pretreatment attenuates MDMA-induced dopamine release in the striatum as measured by microdialysis. Life Sciences, 47(26), 2401-2408. https://doi.org/10.1016/0024-3205(90)90484-9

Nash, J. F., \& Brodkin, J. (1991). Microdialysis studies on 3,4- 
methylenedioxymethamphetamine-induced dopamine release: effect of dopamine uptake inhibitors. J. Pharmacol. Exp. Ther., 259(2), 820-825. Retrieved from http://jpet.aspetjournals.org/content/259/2/820.short

Nestler, E. J. (2008). Review. Transcriptional mechanisms of addiction: role of DeltaFosB. Philosophical Transactions of the Royal Society of London. Series B, Biological Sciences, 363(1507), 3245-3255. https://doi.org/10.1098/rstb.2008.0067

Nestler, E. J. (2012). Transcriptional mechanisms of drug addiction. Clinical Psychopharmacology and Neuroscience: The Official Scientific Journal of the Korean College of Neuropsychopharmacology, 10(3), 136-143. https://doi.org/10.9758/cpn.2012.10.3.136

Nestler, E. J., Kelz, M. B., \& Chen, J. (1999). $\triangle$ FosB: a molecular mediator of long-term neural and behavioral plasticity. Brain Research, 835(1), 10-17. https://doi.org/10.1016/S00068993(98)01191-3

Niessen, W. M. A. (2006). Liquid chromatography-mass spectrometry. CRC press.

Ning, T., Leng, C., Chen, L., Ma, B., \& Gong, X. (2018). Metabolomics analysis of serum in a rat heroin self-administration model undergoing reinforcement based on $1 \mathrm{H}$-nuclear magnetic resonance spectra. BMC Neuroscience, $19(1), \quad 4$. https://doi.org/10.1186/s12868-018-0404-5

Nomura, M., Izaki, Y., Takita, M., Tanaka, J., \& Hori, K. (2004). Extracellular level of basolateral amygdalar dopamine responding to reversal of appetitive-conditioned discrimination in young and old rats. Brain Research, 1018(2), 241-246. https://doi.org/10.1016/J.BRAINRES.2004.05.077

Nye, H. E., Hope, B. T., Kelz, M. B., Iadarola, M. J., \& Nestler, E. J. (1995). Pharmacological studies of the regulation of chronic FOS-related antigen induction by cocaine in the striatum and nucleus accumbens. Journal of Pharmacology and Experimental Therapeutics, 275(3).

O’Brien, C. P., Childress, A. R., Ehrman, R. N., \& Robbins, S. J. (1998). Conditioning factors in drug abuse: can they explain compulsion? Journal of Psychopharmacology (Oxford, England), 12(1), 15-22. https://doi.org/10.1177/026988119801200103

O'Brien, C. P., \& Gardner, E. L. (2005). Critical assessment of how to study addiction and its treatment: Human and non-human animal models. Pharmacology \& Therapeutics, 108(1), 18-58. https://doi.org/10.1016/J.PHARMTHERA.2005.06.018

O'Connor, E. C., Chapman, K., Butler, P., \& Mead, A. N. (2011). The predictive validity of the rat self-administration model for abuse liability. Neuroscience and Biobehavioral Reviews, 35(3), 912-938. https://doi.org/10.1016/j.neubiorev.2010.10.012

O’Dea, D., \& Thompson, G. (2007). Report on Tobacco Taxation in New Zealand.

O’Dell, L. E., Chen, S. A., Smith, R. T., Specio, S. E., Balster, R. L., Paterson, N. E., ... Koob, G. F. (2007). Extended Access to Nicotine Self-Administration Leads to Dependence: Circadian Measures, Withdrawal Measures, and Extinction Behavior in Rats. Journal of Pharmacology and Experimental Therapeutics, 320(1), 180-193. https://doi.org/10.1124/jpet.106.105270

O’Hearn, E., Battaglia, G., De Souza, E. B., Kuhar, M. J., \& Molliver, M. E. (1988). Methylenedioxyamphetamine (MDA) and methylenedioxymethamphetamine (MDMA) cause selective ablation of serotonergic axon terminals in forebrain: immunocytochemical evidence for neurotoxicity. J. Neurosci., 8(8), 2788-2803. Retrieved from http://www.jneurosci.org/content/8/8/2788.short

O'Shea, E., Escobedo, I., Orio, L., Sanchez, V., Navarro, M., Green, A. R., \& Colado, M. I. (2005). Elevation of Ambient Room Temperature has Differential Effects on MDMAInduced 5-HT and Dopamine Release in Striatum and Nucleus Accumbens of Rats. Neuropsychopharmacology, 30(7), 1312-1323. https://doi.org/10.1038/sj.npp.1300673 
Oakly, A. C., Brox, B. W., Schenk, S., \& Ellenbroek, B. A. (2013). A genetic deletion of the serotonin transporter greatly enhances the reinforcing properties of MDMA in rats. Molecular Psychiatry, 19(5), 534-535. https://doi.org/10.1038/mp.2013.75

Olds, J. (1956). Pleasure centers in the brain. Scientific American, 195(4), 105-117.

Olds, J., \& Milner, P. M. (1954). Positive reinforcement produced by electrical stimulation of septal area and other regions of rat brain. Journal of Comparative and Physiological Psychology, 47(6), 419.

Oliver, S. G., Winson, M. K., Kell, D. B., \& Baganz, F. (1998). Systematic functional analysis of the yeast genome. Trends in Biotechnology, 16(9), 373-378. https://doi.org/10.1016/S0167-7799(98)01214-1

Olmstead, M. C., Lafond, M. V., Everitt, B. J., \& Dickinson, A. (2001). Cocaine seeking by rats is a goal-directed action. Behavioral Neuroscience, 115(2), 394-402. https://doi.org/10.1037/0735-7044.115.2.394

Overton, D. A. (1987). Applications and limitations of the drug discrimination method for the study of drug abuse. In Methods of assessing the reinforcing properties of abused drugs (pp. 291-340). Springer.

Panlilio, L. V., \& Goldberg, S. R. (2007). Self-administration of drugs in animals and humans as a model and an investigative tool. Addiction, 102(12), 1863-1870. https://doi.org/10.1111/j.1360-0443.2007.02011.x

Panos, J. J., \& Baker, L. E. (2010). An in vivo microdialysis assessment of concurrent MDMA and cocaine administration in Sprague-Dawley rats. Psychopharmacology, 209(1), 95102. https://doi.org/10.1007/s00213-009-1774-2

Park, W.-K., Bari, A. A., Jey, A. R., Anderson, S. M., Spealman, R. D., Rowlett, J. K., \& Pierce, R. C. (2002). Cocaine administered into the medial prefrontal cortex reinstates cocaine-seeking behavior by increasing AMPA receptor-mediated glutamate transmission in the nucleus accumbens. The Journal of Neuroscience: The Official Journal of the Society for Neuroscience, 22(7), 2916-2925. https://doi.org/20026235

Parkinson, J. A., Olmstead, M. C., Burns, L. H., Robbins, T. W., \& Everitt, B. J. (1999). Dissociation in effects of lesions of the nucleus accumbens core and shell on appetitive pavlovian approach behavior and the potentiation of conditioned reinforcement and locomotor activity by D-amphetamine. The Journal of Neuroscience: The Official Journal of the Society for Neuroscience, 19(6), 2401-2411. Retrieved from http://www.ncbi.nlm.nih.gov/pubmed/10066290

Parrott, A. C. (2005). Chronic tolerance to recreational MDMA (3,4methylenedioxymethamphetamine) or Ecstasy. Journal of Psychopharmacology (Oxford, England), 19(1), 71-83. https://doi.org/10.1177/0269881105048900

Parrott, A. C. (2006). MDMA in humans: factors which affect the neuropsychobiological profiles of recreational ecstasy users, the integrative role of bioenergetic stress. Journal of Psychopharmacology, 20(2), 147-163. https://doi.org/10.1177/0269881106063268

Parrott, A. C. (2013). Human psychobiology of MDMA or 'Ecstasy': an overview of 25 years of empirical research. Human Psychopharmacology: Clinical and Experimental, 28(4), 289-307. https://doi.org/10.1002/hup.2318

Parrott, A. C., Lees, A., Garnham, N. J., Jones, M., \& Wesnes, K. (1998). Cognitive performance in recreational users of MDMA or "ecstasy": evidence for memory deficits. Journal of Psychopharmacology, 12(1), 79-83. https://doi.org/10.1177/026988119801200110

Parrott, A. C., Sisk, E., \& Turner, J. J. D. (2000). Psychobiological problems in heavy 'ecstasy' (MDMA) polydrug users. Drug and Alcohol Dependence, 60(1), 105-110. https://doi.org/10.1016/S0376-8716(00)80013-7

Parsons, L. H., \& Justice, J. B. (1993). Serotonin and Dopamine Sensitization in the Nucleus 
Accumbens, Ventral Tegmental Area, and Dorsal Raphe Nucleus Following Repeated Cocaine Administration. Journal of Neurochemistry, 61(5), 1611-1619. https://doi.org/10.1111/j.1471-4159.1993.tb09794.x

Paterson, N. E., \& Markou, A. (2003). Increased motivation for self-administered cocaine after escalated cocaine intake. NeuroReport, 14(17), 2229-2232. https://doi.org/10.1097/00001756-200312020-00019

Patti, G. J., Yanes, O., \& Siuzdak, G. (2012). Metabolomics: the apogee of the omics trilogy. Nature Reviews Molecular Cell Biology, 13(4), 263-269. https://doi.org/10.1038/nrm3314

Paulson, P. E., \& Robinson, T. E. (1991). Sensitization to systemic amphetamine produces an enhanced locomotor response to a subsequent intra-accumbens amphetamine challenge in rats. Psychopharmacology, 104(1), 140-141. https://doi.org/10.1007/BF02244569

Paulson, P. E., \& Robinson, T. E. (1995). Amphetamine-Induced time-dependent sensitization of dopamine neurotransmission in the dorsal and ventral striatum: A microdialysis study in behaving rats. Synapse, 19(1), 56-65. https://doi.org/10.1002/syn.890190108

Paxinos, G., \& Watson, C. (2005). The rat brain in sterotaxic coordinates (5th ed.). Academic Press. https://doi.org/10.1017/CBO9781107415324.004

Peakman, M.-C., Colby, C. R., Perrotti, L. I., Tekumalla, P., Carle, T. L., Ulery, P. G., ... Schaeffer, E. (2003). Inducible, brain region-specific expression of a dominant negative mutant of c-Jun in transgenic mice decreases sensitivity to cocaine. Brain Research, 970(1-2), 73-86. https://doi.org/10.1016/S0006-8993(03)02230-3

Pelloux, Y., Everitt, B. J., \& Dickinson, A. (2007). Compulsive drug seeking by rats under punishment: effects of drug taking history. Psychopharmacology, 194(1), 127-137. https://doi.org/10.1007/s00213-007-0805-0

Peltier, R., \& Schenk, S. (1993). Effects of serotonergic manipulations on cocaine selfadministration in rats. Psychopharmacology, 110(4), 390-394. https://doi.org/10.1007/BF02244643

Peroutka, S. J., Newman, H., \& Harris, H. (1988). Subjective effects of 3, 4methylenedioxymethamphetamine in recreational users. Neuropsychopharmacology, 1(4), 273-277.

Perrotti, L. I., Bolaños, C. A., Choi, K.-H., Russo, S. J., Edwards, S., Ulery, P. G., ... Barrot, M. (2005). $\triangle$ FosB accumulates in a GABAergic cell population in the posterior tail of the ventral tegmental area after psychostimulant treatment. European Journal of Neuroscience, 21(10), 2817-2824. https://doi.org/10.1111/j.1460-9568.2005.04110.x

Perrotti, L. I., Hadeishi, Y., Ulery, P. G., Barrot, M., Monteggia, L., Duman, R. S., \& Nestler, E. J. (2004). Induction of $\Delta$ FosB in Reward-Related Brain Structures after Chronic Stress. The Journal of Neuroscience, 24(47), 10594 LP - 10602. https://doi.org/10.1523/JNEUROSCI.2542-04.2004

Perrotti, L. I., Weaver, R. R., Robison, B., Renthal, W., Maze, I., Yazdani, S., .. Nestler, E. J. (2008). Distinct patterns of $\Delta$ FosB induction in brain by drugs of abuse. Synapse, 62(5), 358-369. https://doi.org/10.1002/syn.20500

Perry, J. L., Morgan, A. D., Anker, J. J., Dess, N. K., \& Carroll, M. E. (2006). Escalation of i.v. cocaine self-administration and reinstatement of cocaine-seeking behavior in rats bred for high and low saccharin intake. Psychopharmacology, 186(2), 235-245. https://doi.org/10.1007/s00213-006-0371-x

Perugini, M., \& Vezina, P. (1994). Amphetamine administered to the ventral tegmental area sensitizes rats to the locomotor effects of nucleus accumbens amphetamine. Journal of Pharmacology and Experimental Therapeutics, 270(2), 690-696.

Pettit, H. O., \& Justice, J. B. (1991). Effect of dose on cocaine self-administration behavior and dopamine levels in the nucleus accumbens. Brain Research, 539(1), 94-102. 
https://doi.org/10.1016/0006-8993(91)90690-W

Pettit, H. O., Pan, H.-T., Parsons, L. H., \& Justice, J. B. (1990). Extracellular Concentrations of Cocaine and Dopamine Are Enhanced During Chronic Cocaine Administration. Journal of Neurochemistry, 55(3), 798-804. https://doi.org/10.1111/j.14714159.1990.tb04562.x

Pfaus, J. G., Damsma, G., Nomikos, G. G., Wenkstern, D. G., Blaha, C. D., Phillips, A. G., \& Fibiger, H. C. (1990). Sexual behavior enhances central dopamine transmission in the male rat. Brain Research, 530(2), 345-348. https://doi.org/10.1016/0006-8993(90)913095

Phillips, A. G., Mora, F., \& Rolls, E. T. (1981). Intracerebral self-administration of amphetamine by rhesus monkeys. Neuroscience Letters, 24(1), 81-86. https://doi.org/10.1016/0304-3940(81)90363-3

Phillips, G. D., Howes, S. R., Whitelaw, R. B., Robbins, T. W., \& Everitt, B. J. (1994). Isolation rearing impairs the reinforcing efficacy of intravenous cocaine or intra-accumbensdamphetamine: impaired response to intra-accumbens D1 and D2/D3 dopamine receptor antagonists. Psychopharmacology, 115(3), 419-429. https://doi.org/10.1007/BF02245085

Phillips, G. D., Robbins, T. W., \& Everitt, B. J. (1994). Bilateral intra-accumbens selfadministration ofd-amphetamine: Antagonism with intra-accumbens SCH-23390 and sulpiride. Psychopharmacology, 114(3), 477-485. https://doi.org/10.1007/BF02249339

Phillips, P. E. M., Stuber, G. D., Heien, M. L. A. V, Wightman, R. M., \& Carelli, R. M. (2003). Subsecond dopamine release promotes cocaine seeking. Nature, 422(6932), 614-618. https://doi.org/10.1038/nature01476

Piazza, P. V., Deminière, J. M., le Moal, M., \& Simon, H. (1990). Stress- and pharmacologically-induced behavioral sensitization increases vulnerability to acquisition of amphetamine self-administration. Brain Research, 514(1), 22-26. https://doi.org/10.1016/0006-8993(90)90431-A

Piazza, P. V., Deminière, J. M., Le Moal, M., \& Simon, H. (1989). Factors That Predict Individual Vulnerability to Amphetamine Self-Administration. Science, 245(4925), 1511-1513. https://doi.org/10.1126/science.2781295

Piazza, P. V., \& Le Moal, M. (1996). Pathophysiological Basis of Vulnerability to Drug Abuse: Role of an Interaction Between Stress, Glucocorticoids, and Dopaminergic Neurons. Annual Review of Pharmacology and Toxicology, 36(1), 359-378. https://doi.org/10.1146/annurev.pa.36.040196.002043

Piazza, P. V., Maccari, S., Deminière, J. M., Le Moal, M., Mormede, P., \& Simon, H. (1991). Corticosterone levels determine individual vulnerability to amphetamine selfadministration. Proceedings of the National Academy of Sciences, 88(6), 2088-2092. https://doi.org/10.1073/pnas.88.6.2088

Pich, E. M., Pagliusi, S. R., Tessari, M., Talabot-Ayer, D., van Huijsduijnen, R. H., \& Chiamulera, C. (1997). Common Neural Substrates for the Addictive Properties of Nicotine and Cocaine. Science, 275(5296), 83 LP - 86.

Pickens, R., \& Harris, W. C. (1968). Self-administration of d-amphetamine by rats. Psychopharmacologia, 12(2), 158-163. https://doi.org/10.1007/BF00401545

Pierce, R. C., \& Kalivas, P. W. (1995). Amphetamine produces sensitized increases in locomotion and extracellular dopamine preferentially in the nucleus accumbens shell of rats administered repeated cocaine. Journal of Pharmacology and Experimental Therapeutics, 275(2), 1019-1029.

Pierce, R. C., \& Kalivas, P. W. (1997). A circuitry model of the expression of behavioral sensitization to amphetamine-like psychostimulants. Brain Research Reviews, 25(2), 192-216. https://doi.org/10.1016/S0165-0173(97)00021-0 
Pierre, P. J., \& Vezina, P. (1997). Predisposition to self-administer amphetamine: the contribution of response to novelty and prior exposure to the drug. Psychopharmacology, 129(3), 277-284. https://doi.org/10.1007/s002130050191

Pierre, P. J., \& Vezina, P. (1998). D 1 dopamine receptor blockade prevents the facilitation of amphetamine self-administration induced by prior exposure to the drug. Psychopharmacology, 138(2), 159-166. https://doi.org/10.1007/s002130050658

Pijnenburg, A. J. J., Honig, W. M. M., \& Van Rossum, J. M. (1975). Effects of antagonists upon locomotor stimulation induced by injection of dopamine and noradrenaline into the nucleus accumbens of nialamide-pretreated rats. Psychopharmacologia, 41(2), 175-180. https://doi.org/10.1007/BF00421076

Pilla, M., Perachon, S., Sautel, F., Garrido, F., Mann, A., Wermuth, C. G., ... Sokoloff, P. (1999). Selective inhibition of cocaine-seeking behaviour by a partial dopamine D3 receptor agonist. Nature, 400(6742), 371-375. https://doi.org/10.1038/22560

Pluskal, T., Castillo, S., Villar-Briones, A., \& Orešič, M. (2010). MZmine 2: Modular framework for processing, visualizing, and analyzing mass spectrometry-based molecular profile data. BMC Bioinformatics, 11(1), 395. https://doi.org/10.1186/1471-2105-11-395

Porrino, L. J., Ritz, M. C., Goodman, N. L., Sharpe, L. G., Kuhar, M. J., \& Goldberg, S. R. (1989). Differential effects of the pharmacological manipulation of serotonin systems on cocaine and amphetamine self-administration in rats. Life Sciences, 45(17), 1529-1535. Retrieved from http://www.ncbi.nlm.nih.gov/pubmed/2586218

Post, R. M., \& Rose, H. (1976). Increasing effects of repetitive cocaine administration in the rat. Nature, 260(5553), 731-732. https://doi.org/10.1038/260731a0

Ramos-Vara, J. A. (2005). Technical aspects of immunohistochemistry. Veterinary Pathology, 42(4), 405-426. https://doi.org/10.1354/vp.42-4-405

Ramos, M., Goñi-Allo, B., \& Aguirre, N. (2004). Studies on the role of dopamine D1 receptors in the development and expression of MDMA-induced behavioral sensitization in rats. Psychopharmacology, 177(1-2), 100-110. https://doi.org/10.1007/s00213-004-1937-0

Ranaldi, R., Pocock, D., Zereik, R., \& Wise, R. A. (1999). Dopamine Fluctuations in the Nucleus Accumbens during Maintenance, Extinction, and Reinstatement of Intravenous D-Amphetamine Self-Administration. J. Neurosci., 19(10), 4102-4109. Retrieved from http://www.jneurosci.org/content/19/10/4102.short

Ranaldi, R., Wang, Z., \& Woolverton, W. L. (2001). Reinforcing effects of D2 dopamine receptor agonists and partial agonists in rhesus monkeys. Drug and Alcohol Dependence, 64(2), 209-217. https://doi.org/10.1016/S0376-8716(01)00124-7

Rantalainen, M., Cloarec, O., Ebbels, T. M., Lundstedt, T., Nicholson, J. K., Holmes, E., \& Trygg, J. (2008). Piecewise multivariate modelling of sequential metabolic profiling data. BMC Bioinformatics, 9, 105. https://doi.org/10.1186/1471-2105-9-105

Ratzenboeck, E., Saria, A., Kriechbaum, N., \& Zernig, G. (2001). Reinforcing effects of MDMA ("ecstasy") in drug-naive and cocaine-trained rats. Pharmacology, 62(3), 138144. https://doi.org/56086

Renthal, W., Carle, T. L., Maze, I., Covington, H. E., Truong, H.-T., Alibhai, I. N., ... Nestler, E. J. (2008). FosB Mediates Epigenetic Desensitization of the c-fos Gene After Chronic Amphetamine Exposure. Journal of Neuroscience, 28(29), 7344-7349. https://doi.org/10.1523/JNEUROSCI.1043-08.2008

Reveron, M. E., Maier, E. Y., \& Duvauchelle, C. L. (2010). Behavioral, thermal and neurochemical effects of acute and chronic 3,4-methylenedioxymethamphetamine ("Ecstasy") self-administration. Behav Brain Res, 207(2), 500-507. https://doi.org/10.1016/j.bbr.2009.10.038

Ricaurte, G. A., DeLanney, L. E., Irwin, I., \& Langston, J. W. (1988). Toxic effects of MDMA on central serotonergic neurons in the primate: importance of route and frequency of drug 
administration. Brain Research, 446(1), 165-168. https://doi.org/10.1016/00068993(88)91309-1

Ricaurte, G. A., Martello, A., Katz, J., \& Martello, M. (1992). Lasting effects of (+-)-3,4methylenedioxymethamphetamine (MDMA) on central serotonergic neurons in nonhuman primates: neurochemical observations. J. Pharmacol. Exp. Ther., 261(2), 616622. Retrieved from http://jpet.aspetjournals.org/content/261/2/616.short

Ricaurte, G. A., McCann, U. D., Szabo, Z., \& Scheffel, U. (2000). Toxicodynamics and longterm toxicity of the recreational drug, 3,4-methylenedioxymethamphetamine (MDMA, 'Ecstasy'). Toxicology Letters, 112-113, 143-146. https://doi.org/10.1016/S03784274(99)00216-7

Richardson, N. R., \& Roberts, D. C. S. (1991). Fluoxetine pretreatment reduces breaking points on a progressive ratio schedule reinforced by intravenous cocaine self-administration in the rat. Life Sciences, 49(11), 833-840.

Risner, M. E., \& Jones, B. E. (1975). Self-administration of CNS stimulants by dog. Psychopharmacologia, 43(3), 207-213. https://doi.org/10.1007/BF00429252

Risner, M. E., \& Jones, B. E. (1976). Role of noradrenergic and dopaminergic processes in amphetamine self-administration. Pharmacology Biochemistry and Behavior, 5(4), 477482. https://doi.org/10.1016/0091-3057(76)90113-1

Ritz, M. C., \& Kuhar, M. J. (1989). Relationship between self-administration of amphetamine and monoamine receptors in brain: comparison with cocaine. Journal of Pharmacology and Experimental Therapeutics, 248(3), 1010-1017.

Robbins, T. W., \& Everitt, B. J. (1999). Drug addiction: bad habits add up. Nature, 398(6728), 567-570. https://doi.org/10.1038/19208

Roberts, A. J., Heyser, C. J., Cole, M., Griffin, P., \& Koob, G. F. (2000). Excessive Ethanol Drinking Following a History of Dependence Animal Model of Allostasis. Neuropsychopharmacology, 22(6), 581-594. https://doi.org/10.1016/S0893133X(99)00167-0

Roberts, D. C. S., Corcoran, M. E., \& Fibiger, H. C. (1977). On the role of ascending catecholaminergic systems in intravenous self-administration of cocaine. Pharmacology Biochemistry and Behavior, 6(6), 615-620. https://doi.org/10.1016/0091-3057(77)900843

Roberts, D. C. S., \& Koob, G. F. (1982). Disruption of cocaine self-administration following 6-hydroxydopamine lesions of the ventral tegmental area in rats. Pharmacology Biochemistry and Behavior, 17(5), 901-904. https://doi.org/10.1016/00913057(82)90469-5

Roberts, D. C. S., Koob, G. F., Klonoff, P., \& Fibiger, H. C. (1980). Extinction and recovery of cocaine self-administration following 6-hydroxydopamine lesions of the nucleus accumbens. Pharmacology Biochemistry and Behavior, 12(5), 781-787. https://doi.org/10.1016/0091-3057(80)90166-5

Roberts, D. C. S., Phelan, R., Hodges, L. M., Hodges, M. M., Bennett, B., Childers, S., \& Davies, H. (1999). Self-administration of cocaine analogs by rats. Psychopharmacology, 144(4), 389-397. https://doi.org/10.1007/s002130051022

Robinson, D. L., Venton, B. J., Heien, M. L. A. V, \& Wightman, R. M. (2003). Detecting Subsecond Dopamine Release with Fast-Scan Cyclic Voltammetry in Vivo. Clinical Chemistry, 49(10), 1763-1773. https://doi.org/10.1373/49.10.1763

Robinson, T. E. (1984). Behavioral sensitization: Characterization of enduring changes in rotational behavior produced by intermittent injections of amphetamine in male and female rats. Psychopharmacology, 84(4), 466-475. https://doi.org/10.1007/BF00431451

Robinson, T. E., \& Becker, J. B. (1986). Enduring changes in brain and behavior produced by chronic amphetamine administration: A review and evaluation of animal models of 
amphetamine psychosis. Brain Research Reviews, 11(2), 157-198. https://doi.org/10.1016/0165-0173(86)90002-0

Robinson, T. E., \& Berridge, K. C. (1993). The neural basis of drug craving: An incentivesensitization theory of addiction. Brain Research Reviews, 18(3), 247-291. https://doi.org/10.1016/0165-0173(93)90013-P

Robinson, T. E., \& Berridge, K. C. (2000). The psychology and neurobiology of addiction: an incentive-sensitization view. Addiction, 95(8s2), 91-117. https://doi.org/10.1046/j.13600443.95.8s2.19.x

Robinson, T. E., \& Berridge, K. C. (2001). Incentive-sensitization and addiction. Addiction, 96(1), 103-114. https://doi.org/10.1046/j.1360-0443.2001.9611038.x

Robinson, T. E., \& Berridge, K. C. (2008). The incentive sensitization theory of addiction: some current issues. Philosophical Transactions of the Royal Society B: Biological Sciences, 363(1507), 3137-3146. https://doi.org/10.1098/rstb.2008.0093

Robinson, T. E., Jurson, P. A., Bennett, J. A., \& Bentgen, K. M. (1988). Persistent sensitization of dopamine neurotransmission in ventral striatum (nucleus accumbens) produced by prior experience with (+)-amphetamine: a microdialysis study in freely moving rats. Brain Research, 462(2), 211-222. https://doi.org/10.1016/0006-8993(88)90549-5

Robinson, T. E., \& Kolb, B. (2004). Structural plasticity associated with exposure to drugs of abuse. Neuropharmacology, 47, 33-46. https://doi.org/10.1016/J.NEUROPHARM.2004.06.025

Robison, A. J., \& Nestler, E. J. (2011). Transcriptional and epigenetic mechanisms of addiction. Nature Reviews Neuroscience, 12(11), 623-637. https://doi.org/10.1038/nrn3111

Rodd-Henricks, Z. A., Bell, R. L., Kuc, K. A., Murphy, J. M., McBride, W. J., Lumeng, L., \& Li, T.-K. (2002). Effects of Ethanol Exposure on Subsequent Acquisition and Extinction of Ethanol Self-Administration and Expression of Alcohol-Seeking Behavior in Adult Alcohol-Preferring (P) Rats: I. Periadolescent Exposure. Alcoholism: Clinical and Experimental Research, 26(11), 1632-1641. https://doi.org/10.1111/j.15300277.2002.tb02465.x

Rogers, G., Elston, J., Garside, R., Roome, C., Taylor, R. S., Younger, P., ... Somerville, M. (2009). The harmful health effects of recreational ecstasy: a systematic review of observational evidence.

Roiser, J. P., Rogers, R. D., Cook, L. J., \& Sahakian, B. J. (2006). The effect of polymorphism at the serotonin transporter gene on decision-making, memory and executive function in ecstasy users and controls. Psychopharmacology, 188(2), 213-227. https://doi.org/10.1007/s00213-006-0495-z

Routtenberg, A. (1972). Intracranial chemical injection and behavior: A critical review. Behavioral Biology, 7(5), 601-641. https://doi.org/10.1016/S0091-6773(72)80073-7

Rudnick, G., \& Wall, S. C. (1992). The molecular mechanism of "ecstasy" [3,4methylenedioxy-methamphetamine (MDMA)]: serotonin transporters are targets for MDMA-induced serotonin release. Proceedings of the National Academy of Sciences, 89(5), 1817-1821. https://doi.org/10.1073/pnas.89.5.1817

Rusilowicz, M. J., Dickinson, M., Charlton, A. J., O'Keefe, S., \& Wilson, J. (2018). MetaboClust: Using interactive time-series cluster analysis to relate metabolomic data with perturbed pathways. PLOS ONE, 13(10), e0205968. https://doi.org/10.1371/journal.pone.0205968

Sabol, K. E., \& Seiden, L. S. (1998). Reserpine attenuates d-amphetamine and MDMA-induced transmitter release in vivo: a consideration of dose, core temperature and dopamine synthesis. Brain Research, 806(1), 69-78. https://doi.org/10.1016/S0006-8993(98)007203 
Samra, S. N. (2015). Comparative evaluation of open access software used in liquid chromatography-mass spectrometry based untargeted metabolomics. California State University - $\quad$ Sacramento. Retrieved from https://oatd.org/oatd/record?record=handle\%5C:10211.3\%5C\%2F158926\&q=*\%3A*

Samson, H. H., Pfeffer, A. O., \& Tolliver, G. A. (1988). Oral Ethanol Self-administration in Rats: Models of Alcohol-Seeking Behavior. Alcoholism: Clinical and Experimental Research, 12(5), 591-598. https://doi.org/10.1111/j.1530-0277.1988.tb00248.x

Schenk, S. (2002). Effects of GBR 12909, WIN 35,428 and indatraline on cocaine selfadministration and cocaine seeking in rats. Psychopharmacology, 160(3), 263-270. https://doi.org/10.1007/s00213-001-0972-3

Schenk, S. (2011). MDMA ("ecstasy") abuse as an example of dopamine neuroplasticity. Neuroscience and Biobehavioral Reviews, 35(5), 1203-1218. https://doi.org/10.1016/j.neubiorev.2010.12.010

Schenk, S., \& Bradbury, S. (2015). Persistent sensitisation to the locomotor activating effects of MDMA following MDMA self-administration in rats. Pharmacology, Biochemistry, and Behavior, 132, 103-107. https://doi.org/10.1016/j.pbb.2015.03.001

Schenk, S., Colussi-Mas, J., Do, J., \& Bird, J. (2012). Profile of MDMA self-administration from a large cohort of rats: MDMA develops a profile of dependence with extended testing. J Drug Alcohol Res, 1, 1-6.

Schenk, S., Foote, J., Aronsen, D., Bukholt, N., Highgate, Q., Van De Wetering, R., \& Webster, J. (2016). Serotonin antagonists fail to alter MDMA self-administration in rats. Pharmacology Biochemistry and Behavior, 148, 38-45.

Schenk, S., Gittings, D., \& Colussi-Mas, J. (2011). Dopaminergic mechanisms of reinstatement of MDMA-seeking behaviour in rats. British Journal of Pharmacology, 162(8), 17701780. https://doi.org/10.1111/j.1476-5381.2010.01193.x

Schenk, S., Gittings, D., Johnstone, M., \& Daniela, E. (2003). Development, maintenance and temporal pattern of self-administration maintained by ecstasy (MDMA) in rats. Psychopharmacology, 169(1), 21-27. https://doi.org/10.1007/s00213-003-1407-0

Schenk, S., Hely, L., Gittings, D., Lake, B., \& Daniela, E. (2008). Effects of priming injections of MDMA and cocaine on reinstatement of MDMA- and cocaine-seeking in rats. Drug and Alcohol Dependence, 96(3), 249-255. https://doi.org/10.1016/j.drugalcdep.2008.03.014

Schenk, S., Hely, L., Lake, B., Daniela, E., Gittings, D., \& Mash, D. C. (2007). MDMA selfadministration in rats: acquisition, progressive ratio responding and serotonin transporter binding. The European Journal of Neuroscience, 26(11), 3229-3236. https://doi.org/10.1111/j.1460-9568.2007.05932.x

Schenk, S., Lacelle, G., Gorman, K., \& Amit, Z. (1987). Cocaine self-administration in rats influenced by environmental conditions: implications for the etiology of drug abuse. Neuroscience Letters, 81(1-2), 227-231. https://doi.org/10.1016/0304-3940(87)91003-2

Schenk, S., \& Partridge, B. (2001). Influence of a conditioned light stimulus on cocaine selfadministration in rats. Psychopharmacology, 154(4), 390-396. https://doi.org/10.1007/s002130000608

Schenk, S., Robinson, B., \& Amit, Z. (1988). Housing conditions fail to affect the intravenous self-administration of amphetamine. Pharmacology Biochemistry and Behavior, 31(1), 59-62. https://doi.org/10.1016/0091-3057(88)90311-5

Schenk, S., Snow, S., \& Horger, B. A. (1991). Pre-exposure to amphetamine but not nicotine sensitizes rats to the motor activating effect of cocaine. Psychopharmacology, 103(1), 6266. https://doi.org/10.1007/BF02244075

Schenk, S., Valadez, A., McNamara, C. G., House, D. T., Higley, D., Bankson, M. G., ... Horger, B. A. (1993). Development and expression of sensitization to cocaine's 
reinforcing properties: role of NMDA receptors. Psychopharmacology, 111(3), 332-338. https://doi.org/10.1007/BF02244949

Schifano, F., Oyefeso, A., Corkery, J., Cobain, K., Jambert-Gray, R., Martinotti, G., \& Ghodse, A. H. (2003). Death rates from ecstasy (MDMA, MDA) and polydrug use in England and Wales 1996-2002. Human Psychopharmacology, 18(7), 519-524. https://doi.org/10.1002/hup.528

Schindler, C. W., Baumann, M. H., Thorndike, E. B., Blough, B. E., \& Goldberg, S. R. (2012). Effects of 3, 4-methylenedioxymethamphetamine (MDMA) and its metabolites on cardiovascular function in rats. Federation of American Societies for Experimental Biology.

Schindler, C. W., \& Carmona, G. N. (2002). Effects of dopamine agonists and antagonists on locomotor activity in male and female rats. Pharmacology Biochemistry and Behavior, 72(4), 857-863. https://doi.org/10.1016/S0091-3057(02)00770-0

Schindler, C. W., Cogan, E. S., Thorndike, E. B., \& Panlilio, L. V. (2011). Rapid delivery of cocaine facilitates acquisition of self-administration in rats: An effect masked by paired stimuli. Pharmacology Biochemistry and Behavior, 99(3), 301-306. Retrieved from https://www.sciencedirect.com/science/article/pii/S0091305711001389\#bb0160

Schindler, C. W., Panlilio, L. V., \& Goldberg, S. R. (2002). Second-order schedules of drug self-administration in animals. Psychopharmacology, 163(3-4), 327-344. https://doi.org/10.1007/s00213-002-1157-4

Schmidt, C. J. (1987). Neurotoxicity of the psychedelic amphetamine, methylenedioxymethamphetamine. J. Pharmacol. Exp. Ther., 240(1), 1-7. Retrieved from http://jpet.aspetjournals.org/content/240/1/1.short

Schmidt, C. J., Levin, J. A., \& Lovenberg, W. (1987). In vitro and in vivo neurochemical effects of methylenedioxymethamphetamine on striatal monoaminergic systems in the rat brain. Biochemical Pharmacology, 36(5), 747-755. https://doi.org/10.1016/00062952(87)90729-5

Schmidt, C. J., Sullivan, C. K., \& Fedayal, G. M. (1994). Blockade of Striatal 5Hydroxytryptmine2 Receptors Reduces the Increase in Extracellullar Concentrations of Dopamine Produced by the Amphhetamine analogue 3,4Methylenedioxymethamphetamine. Journal of Neurochemistry, 62(4), 1382-1389. https://doi.org/10.1046/j.1471-4159.1994.62041382.x

Schoenbaum, G., Saddoris, M. P., Ramus, S. J., Shaham, Y., \& Setlow, B. (2004). Cocaineexperienced rats exhibit learning deficits in a task sensitive to orbitofrontal cortex lesions. European Journal of Neuroscience, 19(7), 1997-2002. https://doi.org/10.1111/j.14609568.2004.03274.x

Schuldiner, S., Steiner-Mordoch, S., Yelin, R., Wall, S. C., \& Rudnick, G. (1993). Amphetamine derivatives interact with both plasma membrane and secretory vesicle biogenic amine transporters. Mol. Pharmacol., 44(6), 1227-1231. Retrieved from http://molpharm.aspetjournals.org/content/44/6/1227.short

Schultz, W. (1998). Predictive Reward Signal of Dopamine Neurons. Journal of Neurophysiology, 80(1), 1-27. https://doi.org/10.1152/jn.1998.80.1.1

Schultz, W., Dayan, P., \& Montague, P. R. (1997). A neural substrate of prediction and reward. Science (New York, N.Y.), 275(5306), 1593-1599. https://doi.org/10.1126/science.275.5306.1593

Schuster, C. R., \& Thompson, T. (1969). Self administration of and behavioral dependence on drugs. Annual Review of Pharmacology, 9(1), 483-502.

Schuster, C. R., \& Woods, J. H. (1968). The conditioned reinforcing effects of stimuli associated with morphine reinforcement. International Journal of the Addictions, 3(1), $223-230$. 
Scorza, M. C., Carrau, C., Silveira, R., Zapata-Torres, G., Cassels, B. K., \& Reyes-Parada, M. (1997). Monoamine Oxidase Inhibitory Properties of Some Methoxylated and Alkylthio Amphetamine Derivatives. Biochemical Pharmacology, 54(12), 1361-1369. https://doi.org/10.1016/S0006-2952(97)00405-X

Screaton, G. R., Cairns, H. S., Sarner, M., Singer, M., Thrasher, A., \& Cohen, S. L. (1992). Hyperpyrexia and rhabdomyolysis after MDMA (" ecstasy") abuse. The Lancet, 339(8794), 677-678.

See, R. E., Fuchs, R. A., Ledford, C. C., \& Mclaughlin, J. (2006). Drug Addiction, Relapse, and the Amygdala. Annals of the New York Academy of Sciences, 985(1), 294-307. https://doi.org/10.1111/j.1749-6632.2003.tb07089.x

See, R. E., Kruzich, P. J., \& Grimm, J. W. (2001). Dopamine, but not glutamate, receptor blockade in the basolateral amygdala attenuates conditioned reward in a rat model of relapse to cocaine-seeking behavior. Psychopharmacology, 154(3), 301-310. https://doi.org/10.1007/s002130000636

Seevers, M. H., \& Tatum, A. L. (1931). CHRONIC EXPERIMENTAL BARBITAL POISONING. J. Pharmacol. Exp. Ther., 42(2), 217-231. Retrieved from http://jpet.aspetjournals.org/content/42/2/217.short

Self, D. W., Belluzzi, J. D., Kossuth, S., \& Stein, L. (1996). Self-administration of the D1 agonist SKF 82958 is mediated by D1, not D2, receptors. Psychopharmacology, 123(4), 303-306. https://doi.org/10.1007/BF02246638

Self, D. W., \& Stein, L. (1992). The D1 agonists SKF 82958 and SKF 77434 are selfadministered by rats. Brain Research, 582(2), 349-352. https://doi.org/10.1016/00068993(92)90155-3

Semple, D. M., Ebmeier, K. P., Glabus, M. F., O’Carroll, R. E., \& Johnstone, E. C. (1999). Reduced in vivo binding to the serotonin transporter in the cerebral cortex of MDMA ('ecstasy') users. The British Journal of Psychiatry, 175(1), 63-69. https://doi.org/10.1192/bjp.175.1.63

Series, H., Boeles, S., Dorkins, E., \& Peveler, R. (1994). Psychiatric complications of "Ecstasy" use. Journal of Psychopharmacology (Oxford, England), 8(1), 60-61. https://doi.org/10.1177/026988119400800110

Shah, A., Silverstein, P. S., Singh, D. P., \& Kumar, A. (2012). Involvement of metabotropic glutamate receptor 5, AKT/PI3K Signaling and NF- $\mathrm{KB}$ pathway in methamphetaminemediated increase in IL-6 and IL-8 expression in astrocytes. Journal of Neuroinflammation, 9(1), 547. https://doi.org/10.1186/1742-2094-9-52

Shaham, Y., Rajabi, H., \& Stewart, J. (1996). Relapse to heroin-seeking in rats under opioid maintenance: the effects of stress, heroin priming, and withdrawal. The Journal of Neuroscience: The Official Journal of the Society for Neuroscience, 16(5), 1957-1963. https://doi.org/10.1523/JNEUROSCI.16-05-01957.1996

Shaham, Y., Shalev, U., Lu, L., De Wit, H., \& Stewart, J. (2003). The reinstatement model of drug relapse: history, methodology and major findings. Psychopharmacology, 168(1-2), 3-20. https://doi.org/10.1007/s00213-002-1224-x

Shaham, Y., \& Stewart, J. (1995). Stress reinstates heroin-seeking in drug-free animals: An effect mimicking heroin, not withdrawal. Psychopharmacology, 119(3), 334-341. https://doi.org/10.1007/BF02246300

Shankaran, M., \& Gudelsky, G. A. (1999). A neurotoxic regimen of MDMA suppresses behavioral, thermal and neurochemical responses to subsequent MDMA administration. Psychopharmacology, 147(1), 66-72. https://doi.org/10.1007/s002130051143

Sharp, T., Zetterström, T., Ljungberg, T., \& Ungerstedt, U. (1987). A direct comparison of amphetamine-induced behaviours and regional brain dopamine release in the rat using intracerebral dialysis. Brain Research, 401(2), 322-330. https://doi.org/10.1016/0006- 
8993(87)91416-8

Shiflett, M. W., Brown, R. A., \& Balleine, B. W. (2010). Acquisition and Performance of GoalDirected Instrumental Actions Depends on ERK Signaling in Distinct Regions of Dorsal Striatum in Rats. Journal of Neuroscience, 30(8), 2951-2959. https://doi.org/10.1523/JNEUROSCI.1778-09.2010

Shippenberg, T. S., \& Heidbreder, C. A. (1995). Sensitization to the conditioned rewarding effects of cocaine: pharmacological and temporal characteristics. Journal of Pharmacology and Experimental Therapeutics, 273(2).

Shulaev, V. (2006). Metabolomics technology and bioinformatics. Briefings in Bioinformatics, 7(2), 128-139. https://doi.org/10.1093/bib/bbl012

Singer, G., Wallace, M., \& Hall, R. (1982). Effects of dopaminergic nucleus accumbens lesions on the acquisition of schedule induced self injection of nicotine in the rat. Pharmacology Biochemistry and Behavior, 17(3), 579-581. https://doi.org/10.1016/00913057(82)90321-5

Skinner, B. F. (1938). The behavior of organisms: an experimental analysis. Oxford, England: Appleton-Century.

Slack, A., Nana, G., Webster, M., Stokes, F., \& Wu, J. (2009). Costs of Harmful Alcohol and Other Drug Use. Wellington: Businiess and Economic Research Limited.

Smilde, A. K., Westerhuis, J. A., Hoefsloot, H. C. J., Bijlsma, S., Rubingh, C. M., Vis, D. J., ... van der Greef, J. (2010). Dynamic metabolomic data analysis: a tutorial review. Metabolomics, 6(1), 3-17. https://doi.org/10.1007/s11306-009-0191-1

Smith, C. A., Maille, G. O., Want, E. J., Qin, C., Trauger, S. A., Brandon, T. R., ... Siuzdak, G. (2005). METLIN. Therapeutic Drug Monitoring, 27(6), 747-751. https://doi.org/10.1097/01.ftd.0000179845.53213.39

Smith, C. T., Dang, L. C., Cowan, R. L., Kessler, R. M., \& Zald, D. H. (2016). Variability in paralimbic dopamine signaling correlates with subjective responses to d-amphetamine. Neuropharmacology, 108, 394-402. https://doi.org/10.1016/j.neuropharm.2016.05.004

Smith, J. E., Dianna, S. L., Smith, D. G., Leccese, A. P., \& Lyness, W. H. (1986). Dietary tryptophan supplements attenuate amphetamine self-administration in the rat. Pharmacology Biochemistry and Behavior, 25(4), 849-855.

Smith, J. E., Shultz, K., Co, C., Goeders, N. E., \& Dworkin, S. I. (1987). Effects of 5, 7dihydroxytryptamine lesions of the nucleus accumbens on rat inravenous morphine selfadministration. Pharmacology Biochemistry and Behavior, 26(3), 607-612.

Smith, S. G., \& Davis, W. M. (1973). Behavioral control by stimuli associated with acquisition of morphine self-administration. Behavioral Biology, 9(6), 777-780. https://doi.org/10.1016/S0091-6773(73)80139-7

Solomon, R. L., \& Corbit, J. D. (1974). An opponent-process theory of motivation: I. Temporal dynamics of affect. Psychological Review, 81(2), 119-145. https://doi.org/10.1037/h0036128

Song, P., Mabrouk, O. S., Hershey, N. D., \& Kennedy, R. T. (2012). In Vivo Neurochemical Monitoring Using Benzoyl Chloride Derivatization and Liquid Chromatography-Mass Spectrometry. Analytical Chemistry, 84(1), 412-419. https://doi.org/10.1021/ac202794q

Spanos, L. J., \& Yamamoto, B. K. (1989). Acute and subchronic effects of methylenedioxymethamphetamine $[( \pm)$ MDMA $]$ on locomotion and serotonin syndrome behavior in the rat. Pharmacology Biochemistry and Behavior, 32(4), 835-840. https://doi.org/10.1016/0091-3057(89)90044-0

Steinpreis, R. E., \& Salamone, J. D. (1993). The role of nucleus accumbens dopamine in the neurochemical and behavioral effects of phencyclidine: a microdialysis and behavioral study. Brain Research, 612(1-2), 263-270. https://doi.org/10.1016/0006-8993(93)91671$\mathrm{E}$ 
Stewart, J., \& Badiani, A. (1993). Tolerance and sensitization to the behavioral effects of drugs. Behavioural Pharmacology, 4(4), 289-312.

Stewart, J., \& de Wit, H. (1987). Reinstatement of Drug-Taking Behavior as a Method of Assessing Incentive Motivational Properties of Drugs. In Methods of Assessing the Reinforcing Properties of Abused Drugs (pp. 211-227). New York, NY: Springer New York. https://doi.org/10.1007/978-1-4612-4812-5_12

Stewart, J., de Wit, H., \& Eikelboom, R. (1984). Role of unconditioned and conditioned drug effects in the self-administration of opiates and stimulants. Psychological Review, 91(2), 251-268. Retrieved from http://www.ncbi.nlm.nih.gov/pubmed/6571424

Stewart, J., \& Vezina, P. (1987). Environment-specific enhancement of the hyperactivity induced by systemic or intra-VTA morphine injections in rats preexposed to amphetamine. Psychobiology, 15(2), 144-153. https://doi.org/10.3758/bf03333103

Stone, D. M., Stahl, D. C., Hanson, G. R., \& Gibb, J. W. (1986). The effects of 3,4methylenedioxymethamphetamine (MDMA) and 3,4-methylenedioxyamphetamine (MDA) on monoaminergic systems in the rat brain. European Journal of Pharmacology, 128(1-2), 41-48. https://doi.org/10.1016/0014-2999(86)90555-8

Sulzer, D., \& Rayport, S. (1990). Amphetamine and other psychostimulants reduce pH gradients in midbrain dopaminergic neurons and chromaffin granules: A mechanism of action. Neuron, 5(6), 797-808. https://doi.org/10.1016/0896-6273(90)90339-H

Sumner, L. W., Amberg, A., Barrett, D., Beale, M. H., Beger, R., Daykin, C. A., ... Viant, M. R. (2007). Proposed minimum reporting standards for chemical analysis. Metabolomics, 3(3), 211-221. https://doi.org/10.1007/s11306-007-0082-2

Suominen, T., Uutela, P., Ketola, R. A., Bergquist, J., Hillered, L., Finel, M., ... Kostiainen, R. (2013). Determination of Serotonin and Dopamine Metabolites in Human Brain Microdialysis and Cerebrospinal Fluid Samples by UPLC-MS/MS: Discovery of Intact Glucuronide and Sulfate Conjugates. PLoS ONE, 8(6), e68007. https://doi.org/10.1371/journal.pone.0068007

Swartz, M. (2010). HPLC DETECTORS: A BRIEF REVIEW. Journal of Liquid Chromatography \& Related Technologies, 33(9-12), 1130-1150. https://doi.org/10.1080/10826076.2010.484356

Sysi-Aho, M., Katajamaa, M., Yetukuri, L., \& Orešič, M. (2007). Normalization method for metabolomics data using optimal selection of multiple internal standards. $B M C$ Bioinformatics, 8(1), 93. https://doi.org/10.1186/1471-2105-8-93

Szymańska, E., Saccenti, E., Smilde, A. K., \& Westerhuis, J. A. (2012). Double-check: validation of diagnostic statistics for PLS-DA models in metabolomics studies. Metabolomics, 8(S1), 3-16. https://doi.org/10.1007/s11306-011-0330-3

Tan, A., \& Awaiye, K. (2013). Use of internal standards in LC-MS bioanalysis. In Handbook of LC-MS Bioanalysis: Best Practices, Experimental Protocols, and Regulations (pp. 217-227). Wiley Online Library.

Taylor, J. R., \& Horger, B. A. (1999). Enhanced responding for conditioned reward produced by intra-accumbens amphetamine is potentiated after cocaine sensitization. Psychopharmacology, 142(1), 31-40. https://doi.org/10.1007/s002130050859

Taylor, J. R., \& Jentsch, J. D. (2001). Repeated intermittent administration of psychomotor stimulant drugs alters the acquisition of Pavlovian approach behavior in rats: differential effects of cocaine, d-amphetamine and 3,4- methylenedioxymethamphetamine (\&quot;Ecstasy\&quot;). Biological Psychiatry, 50(2), 137-143. https://doi.org/10.1016/s0006-3223(01)01106-4

Tessel, R. E., \& Woods, J. H. (1975). Fenfluramine and N-ethyl amphetamine: Comparison of the reinforcing and rate-decreasing actions in the rhesus monkey. Psychopharmacologia, 43(3), 239-244. https://doi.org/10.1007/BF00429257 
Thomasius, R., Petersen, K., Buchert, R., Andresen, B., Zapletalova, P., Wartberg, L., ... Schmoldt, A. (2003). Mood, cognition and serotonin transporter availability in current and former ecstasy (MDMA) users. Psychopharmacology, 167(1), 85-96. https://doi.org/10.1007/s00213-002-1383-9

Thomasius, R., Zapletalova, P., Petersen, K., Buchert, R., Andresen, B., Wartberg, L., ... Schmoldt, A. (2006). Mood, cognition and serotonin transporter availability in current and former ecstasy (MDMA) users: the longitudinal perspective. Journal of Psychopharmacology (Oxford, England), 20(2), 211-225. https://doi.org/10.1177/0269881106059486

Thompson, T., \& Schuster, C. R. (1964). Morphine self-administration, food-reinforced, and avoidance behaviors in rhesus monkeys. Psychopharmacologia, 5(2), 87-94. https://doi.org/10.1007/BF00413045

Tindell, A. J., Berridge, K. C., Zhang, J., Peciña, S., \& Aldridge, J. W. (2005). Ventral pallidal neurons code incentive motivation: amplification by mesolimbic sensitization and amphetamine. European Journal of Neuroscience, 22(10), 2617-2634. https://doi.org/10.1111/j.1460-9568.2005.04411.x

Topp, L., Hall, W., \& Hando, J. (1997). Is there a dependence syndrome for ecstasy? National Drug and Alcohol Research Centre.

Topp, L., Hando, J., Dillon, P., Roche, A., \& Solowij, N. (1999). Ecstasy use in Australia: patterns of use and associated harm. Drug and Alcohol Dependence, 55(1-2), 105-115. https://doi.org/10.1016/S0376-8716(99)00002-2

Triba, M. N., Le Moyec, L., Amathieu, R., Goossens, C., Bouchemal, N., Nahon, P., ... Savarin, P. (2015). PLS/OPLS models in metabolomics: the impact of permutation of dataset rows on the K-fold cross-validation quality parameters. Molecular BioSystems, 11(1), 13-19. https://doi.org/10.1039/C4MB00414K

Tricomi, E., Balleine, B. W., \& O'Doherty, J. P. (2009). A specific role for posterior dorsolateral striatum in human habit learning. European Journal of Neuroscience, 29(11), 2225-2232. https://doi.org/10.1111/j.1460-9568.2009.06796.x

Trygg, J., \& Wold, S. (2002). Orthogonal projections to latent structures (O-PLS). Journal of Chemometrics, 16(3), 119-128. https://doi.org/10.1002/cem.695

Tzschentke, T. M. (2007). Measuring reward with the conditioned place preference (CPP) paradigm: update of the last decade. Addiction Biology, 12(3-4), 227-462. https://doi.org/10.1111/j.1369-1600.2007.00070.x

Uarrota, V. G., Moresco, R., Coelho, B., Nunes, E. da C., Peruch, L. A. M., Neubert, E. de O., ... Maraschin, M. (2014). Metabolomics combined with chemometric tools (PCA, HCA, PLS-DA and SVM) for screening cassava (Manihot esculenta Crantz) roots during postharvest physiological deterioration. Food Chemistry, 161, 67-78. https://doi.org/10.1016/J.FOODCHEM.2014.03.110

Ulery, P. G., Rudenko, G., \& Nestler, E. J. (2006). Regulation of $\Delta$ FosB Stability by Phosphorylation. The Journal of Neuroscience, 26(19), 5131 LP - 5142. Retrieved from http://www.jneurosci.org/content/26/19/5131.abstract

Ungerstedt, U. (1979). Central dopamine mechanisms and unconditioned behaviour. The Neurobiology of Dopamine, 577-596.

Ungerstedt, U., \& Pycock, C. (1974). Functional correlates of dopamine neurotransmission. Bulletin Der Schweizerischen Akademie Der Medizinischen Wissenschaften, 30(1-3), 4455. Retrieved from http://www.ncbi.nlm.nih.gov/pubmed/4371656

United Nations Office on Drugs and Crime [UNODC]. (2019). World Drug Report 2019.

Uylings, H. B. M., Groenewegen, H. J., \& Kolb, B. (2003). Do rats have a prefrontal cortex? Behavioural Brain Research, 146(1-2), 3-17. https://doi.org/10.1016/J.BBR.2003.09.028 
van de Wetering, R., \& Schenk, S. (2017). Repeated MDMA administration increases MDMAproduced locomotor activity and facilitates the acquisition of MDMA self-administration: role of dopamine D2 receptor mechanisms. Psychopharmacology, 234(7), 1155-1164. https://doi.org/10.1007/s00213-017-4554-4

van de Wetering, R., \& Schenk, S. (2019). Regional changes in $\Delta$ FosB expression in rat brain following MDMA self-administration predict increased sensitivity to effects of locally infused MDMA. Addiction Biology. https://doi.org/10.1111/adb.12814

van den Berg, R. A., Hoefsloot, H. C. J., Westerhuis, J. A., Smilde, A. K., \& van der Werf, M. J. (2006). Centering, scaling, and transformations: improving the biological information content of metabolomics data. BMC Genomics, 7(1), 142. https://doi.org/10.1186/14712164-7-142

Vanderschuren, L. J. M. J., Di Ciano, P., \& Everitt, B. J. (2005). Involvement of the dorsal striatum in cue-controlled cocaine seeking. Journal of Neuroscience, 25(38), 8665-8670.

Vanderschuren, L. J. M. J., \& Everitt, B. J. (2004). Drug Seeking Becomes Compulsive After Prolonged Cocaine Self-Administration. Science, 305(5686), 1017-1019. https://doi.org/10.1126/science.1098975

Vanderschuren, L. J. M. J., \& Kalivas, P. W. (2000). Alterations in dopaminergic and glutamatergic transmission in the induction and expression of behavioral sensitization: a critical review of preclinical studies. Psychopharmacology, 151(2-3), 99-120. https://doi.org/10.1007/s002130000493

Vanderschuren, L. J. M. J., \& Pierce, R. C. (2010). Sensitization Processes in Drug Addiction (pp. 179-195). Springer, Berlin, Heidelberg. https://doi.org/10.1007/7854_2009_21

Vanderschuren, L. J. M. J., Schoffelmeer, A. N. M., Mulder, A. H., \& De Vries, T. J. (1999). Dopaminergic mechanisms mediating the long-term expression of locomotor sensitization following pre-exposure to morphine or amphetamine. Psychopharmacology, 143(3), 244253. https://doi.org/10.1007/s002130050943

Vanover, K. E., Nader, M. A., \& Woolverton, W. L. (1992). Evaluation of the discriminative stimulus and reinforcing effects of sertraline in rhesus monkeys. Pharmacology, Biochemistry, and Behavior, 41(4), 789-793. Retrieved from http://www.ncbi.nlm.nih.gov/pubmed/1594647

Verkes, R. J., Gijsman, H. J., Pieters, M. S. M., Schoemaker, R. C., de Visser, S., Kuijpers, M., ... Cohen, A. F. (2001). Cognitive performance and serotonergic function in users of ecstasy. Psychopharmacology, 153(2), 196-202. https://doi.org/10.1007/s002130000563

Veselkov, K. A., Vingara, L. K., Masson, P., Robinette, S. L., Want, E. J., Li, J. V., ... Nicholson, J. K. (2011). Optimized Preprocessing of Ultra-Performance Liquid Chromatography/Mass Spectrometry Urinary Metabolic Profiles for Improved Information Recovery. Analytical Chemistry, 83(15), 5864-5872. https://doi.org/10.1021/ac201065j

Vezina, P. (1993). Amphetamine injected into the ventral tegmental area sensitizes the nucleus accumbens dopaminergic response to systemic amphetamine: an in vivo microdialysis study in the rat. Brain Research, 605(2), 332-337. https://doi.org/10.1016/00068993(93)91761-G

Vezina, P. (1996). D1 dopamine receptor activation is necessary for the induction of sensitization by amphetamine in the ventral tegmental area. J. Neurosci., 16(7), 24112420. Retrieved from http://www.jneurosci.org/content/16/7/2411.short

Vezina, P. (2004). Sensitization of midbrain dopamine neuron reactivity and the selfadministration of psychomotor stimulant drugs. Neuroscience and Biobehavioral Reviews, 27(8), 827-839. https://doi.org/10.1016/j.neubiorev.2003.11.001

Vezina, P., Kalivas, P. W., \& Stewart, J. (1987). Sensitization occurs to the locomotor effects of morphine and the specific $\mu$ opioid receptor agonist, DAGO, administered repeatedly 
to the ventral tegmental area but not to the nucleus accumbens. Brain Research, 417(1), 51-58. https://doi.org/10.1016/0006-8993(87)90178-8

Vezina, P., \& Leyton, M. (2009). Conditioned cues and the expression of stimulant sensitization in animals and humans. Neuropharmacology, 56, 160-168. https://doi.org/10.1016/J.NEUROPHARM.2008.06.070

Vezina, P., Lorrain, D. S., Arnold, G. M., Austin, J. D., \& Suto, N. (2002). Sensitization of Midbrain Dopamine Neuron Reactivity Promotes the Pursuit of Amphetamine. J. Neurosci., 22(11), 4654-4662. https://doi.org/20026447

Vezina, P., \& Stewart, J. (1984). Conditioning and place-specific sensitization of increases in activity induced by morphine in the VTA. Pharmacology Biochemistry and Behavior, 20(6), 925-934.

Vezina, P., \& Stewart, J. (1989). The effect of dopamine receptor blockade on the development of sensitization to the locomotor activating effects of amphetamine and morphine. Brain Research, 499(1), 108-120. https://doi.org/10.1016/0006-8993(89)91140-2

Volkow, N. D., Koob, G. F., \& McLellan, A. T. (2016). Neurobiologic Advances from the Brain Disease Model of Addiction. New England Journal of Medicine, 374(4), 363-371. https://doi.org/10.1056/NEJMra1511480

Volkow, N. D., Wang, G.-J., Fowler, J. S., Logan, J., Jayne, M., Franceschi, D., ... Pappas, N. (2002). "Nonhedonic" food motivation in humans involves dopamine in the dorsal striatum and methylphenidate amplifies this effect. Synapse, 44(3), 175-180. https://doi.org/10.1002/syn.10075

Voorn, P., Vanderschuren, L. J. M. J., Groenewegen, H. J., Robbins, T. W., \& Pennartz, C. M. A. (2004). Putting a spin on the dorsal-ventral divide of the striatum. Trends in Neurosciences, 27(8), 468-474. https://doi.org/10.1016/J.TINS.2004.06.006

Walker, B. M., \& Koob, G. F. (2007). The ?-Aminobutyric Acid-B Receptor Agonist Baclofen Attenuates Responding for Ethanol in Ethanol-Dependent Rats. Alcoholism: Clinical and Experimental Research, 31(1), 11-18. https://doi.org/10.1111/j.1530-0277.2006.00259.x

Wang, X., Yang, B., Sun, H., \& Zhang, A. (2012). Pattern Recognition Approaches and Computational Systems Tools for Ultra Performance Liquid Chromatography-Mass Spectrometry-Based Comprehensive Metabolomic Profiling and Pathways Analysis of Biological Data Sets. Analytical Chemistry, 84(1), 428-439. https://doi.org/10.1021/ac202828r

Wang, Y., Joharchi, N., Fletcher, P. J., Sellers, E. M., \& Higgins, G. A. (1995). Further studies to examine the nature of dexfenfluramine-induced suppression of heroin self$\begin{array}{lll}\text { administration. } & \text { Psychopharmacology, 134-141. }\end{array}$ https://doi.org/10.1007/BF02246185

Wang, Z., \& Woolverton, W. L. (2007). Estimating the relative reinforcing strength of (+/-)3,4-methylenedioxymethamphetamine (MDMA) and its isomers in rhesus monkeys: comparison to (+)-methamphetamine. Psychopharmacology, 189(4), 483-488. https://doi.org/10.1007/s00213-006-0599-5

Wee, S., Anderson, K. G., Baumann, M. H., Rothman, R. B., Blough, B. E., \& Woolverton, W. L. (2005). Relationship between the serotonergic activity and reinforcing effects of a series of amphetamine analogs. The Journal of Pharmacology and Experimental Therapeutics, 313(2), 848-854. https://doi.org/10.1124/jpet.104.080101

Wee, S., Mandyam, C. D., Lekic, D. M., \& Koob, G. F. (2008). $\alpha 1$-Noradrenergic system role in increased motivation for cocaine intake in rats with prolonged access. European Neuropsychopharmacology, $18(4)$

303-311. https://doi.org/10.1016/j.euroneuro.2007.08.003

Wee, S., \& Woolverton, W. L. (2006). Self-administration of mixtures of fenfluramine and amphetamine by rhesus monkeys. Pharmacology, Biochemistry, and Behavior, 84(2), 
337-343. https://doi.org/10.1016/j.pbb.2006.05.022

Weed, M., \& Woolverton, W. L. (1995). The reinforcing effects of dopamine D1 receptor agonists in rhesus monkeys. J. Pharmacol. Exp. Ther., 275(3), 1367-1374. Retrieved from http://jpet.aspetjournals.org/content/275/3/1367.short

Weeks, J. R. (1962). Experimental morphine addiction: Method for automatic intravenous injections in unrestrained rats. Science, 138(3537), 143-144.

Weeks, J. R., \& Collins, R. J. (1964). Factors affecting voluntary morphine intake in selfmaintained addicted rats. Psychopharmacologia, 6(4), 267-279. https://doi.org/10.1007/BF00413156

Weiss, F. (2005). Neurobiology of craving, conditioned reward and relapse. Curr Opin Pharmacol, 5(1), 9-19. https://doi.org/10.1016/j.coph.2004.11.001

Weiss, F., Maldonado-Vlaar, C. S., Parsons, L. H., Kerr, T. M., Smith, D. L., \& Ben-Shahar, O. (2000). Control of cocaine-seeking behavior by drug-associated stimuli in rats: effects on recovery of extinguished operant-responding and extracellular dopamine levels in amygdala and nucleus accumbens. Proceedings of the National Academy of Sciences of the United States of America, 97(8), 4321-4326. https://doi.org/10.1073/pnas.97.8.4321

Westerink, B. H. C. (1995). Brain microdialysis and its application for the study of animal behaviour. Behavioural Brain Research, 70(2), 103-124. https://doi.org/10.1016/01664328(95)80001-8

Westerink, B. H. C., \& Cremers, T. I. F. H. (2007). Handbook of microdialysis: methods, applications and perspectives (Vol. 16). Academic Press.

Wheelock, A. M., \& Wheelock, C. E. (2013). Trials and tribulations of 'omics data analysis: assessing quality of SIMCA-based multivariate models using examples from pulmonary medicine. Molecular BioSystems, 9(11), 2589. https://doi.org/10.1039/c3mb70194h

White, N. M., \& Milner, P. M. (1992). The Psychobiology of Reinforcers. Annual Review of Psychology, 43(1), 443-471. https://doi.org/10.1146/annurev.ps.43.020192.002303

Whitelaw, R. B., Markou, A., Robbins, T. W., \& Everitt, B. J. (1996). Excitotoxic lesions of the basolateral amygdala impair the acquisition of cocaine-seeking behaviour under a second-order schedule of reinforcement. Psychopharmacology, 127(1-2), 213-224. https://doi.org/10.1007/BF02805996

Wiklund, S., Johansson, E., Sjöström, L., Mellerowicz, E. J., Edlund, U., Shockcor, J. P., ... Trygg, J. (2007). Visualization of GC/TOF-MS-Based Metabolomics Data for Identification of Biochemically Interesting Compounds Using OPLS Class Models. https://doi.org/10.1021/AC0713510

Williams, R. E., Lenz, E. M., Rantalainen, M., \& Wilson, I. D. (2006). The comparative metabonomics of age-related changes in the urinary composition of male Wistar-derived and Zucker (fa/fa) obese rats. Molecular BioSystems, 2(3-4), 193. https://doi.org/10.1039/b517195d

Williamson, S. (1997). Adverse effects of stimulant drugs in a community sample of drug users. Drug and Alcohol Dependence, 44(2-3), 87-94. https://doi.org/10.1016/S03768716(96)01324-5

Wilson, M. A., Ricaurte, G. A., \& Molliver, M. E. (1989). Distinct morphologic classes of serotonergic axons in primates exhibit differential vulnerability to the psychotropic drug 3,4-methylenedioxymethamphetamine. Neuroscience, 28(1), 121-137. https://doi.org/10.1016/0306-4522(89)90237-6

Winger, G., \& Woods, J. H. (1973). The reinforcing property of ethanol in the rhesus monkey: I. Initiation, maintenance and termination of intravenous ethanol-reinforced responding.

Winstanley, C. A., Green, T. A., Theobald, D. E. H., Renthal, W., LaPlant, Q., DiLeone, R. J., ... Nestler, E. J. (2009). $\triangle$ FosB induction in orbitofrontal cortex potentiates locomotor sensitization despite attenuating the cognitive dysfunction caused by cocaine. 
Pharmacology Biochemistry and Behavior, 93(3), 278-284. https://doi.org/10.1016/J.PBB.2008.12.007

Winstanley, C. A., LaPlant, Q., Theobald, D. E. H., Green, T. A., Bachtell, R. K., Perrotti, L. I., ... Nestler, E. J. (2007). $\triangle$ FosB Induction in Orbitofrontal Cortex Mediates Tolerance to Cocaine-Induced Cognitive Dysfunction. The Journal of Neuroscience, 27(39), 10497 LP - 10507.

Wise, R. A. (1980). The dopamine synapse and the notion of 'pleasure centers' in the brain. Trends in Neurosciences, 3(4), 91-95. https://doi.org/10.1016/0166-2236(80)90035-1

Wise, R. A. (1982). Neuroleptics and operant behavior: The anhedonia hypothesis. Behavioral and Brain Sciences, 5(1), 39-53. https://doi.org/10.1017/S0140525X00010372

Wise, R. A. (2000). Addiction becomes a brain disease. Neuron, 26(1), 27-33. https://doi.org/10.1016/s0896-6273(00)81134-4

Wise, R. A. (2008). Dopamine and reward: The anhedonia hypothesis 30 years on. Neurotoxicity Research, 14(2-3), 169-183. https://doi.org/10.1007/BF03033808

Wise, R. A., \& Bozarth, M. A. (1987). A psychomotor stimulant theory of addiction. Psychological Review, 94(4), 469-492. https://doi.org/10.1037/0033-295X.94.4.469

Wise, R. A., Leeb, K., Pocock, D., Newton, P., Burnette, B., \& Justice, J. B. (1995). Fluctuations in nucleus accumbens dopamine concentration during intravenous cocaine self-administration in rats. Psychopharmacology, 120(1), 10-20. https://doi.org/10.1007/BF02246140

Wold, S., Antti, H., Lindgren, F., \& Öhman, J. (1998). Orthogonal signal correction of nearinfrared spectra. Chemometrics and Intelligent Laboratory Systems, 44(1-2), 175-185. https://doi.org/10.1016/S0169-7439(98)00109-9

Wold, S., Sjöström, M., \& Eriksson, L. (2001). PLS-regression: a basic tool of chemometrics. Chemometrics and Intelligent Laboratory Systems, 58(2), 109-130. https://doi.org/10.1016/S0169-7439(01)00155-1

Wolf, M. E. (1998). The role of excitatory amino acids in behavioral sensitization to psychomotor stimulants. Progress in Neurobiology, 54(6), 679-720. https://doi.org/10.1016/S0301-0082(97)00090-7

Wolf, M. E., White, F. J., \& Hu, X.-T. (1994). MK-801 prevents alterations in the mesoaccumbens dopamine system associated with behavioral sensitization to amphetamine. The Journal of Neuroscience: The Official Journal of the Society for Neuroscience, 14(3 Pt 2), 1735-1745. https://doi.org/10.1523/JNEUROSCI.14-0301735.1994

Wong, J.-M. T., Malec, P. A., Mabrouk, O. S., Ro, J., Dus, M., \& Kennedy, R. T. (2016). Benzoyl chloride derivatization with liquid chromatography-mass spectrometry for targeted metabolomics of neurochemicals in biological samples. Journal of Chromatography A, 1446, 78-90. https://doi.org/10.1016/j.chroma.2016.04.006

Woolverton, W. L., Goldberg, L. I., \& Ginos, J. Z. (1984). Intravenous self-administration of dopamine receptor agonists by rhesus monkeys. J. Pharmacol. Exp. Ther., 230(3), 678683. Retrieved from http://jpet.aspetjournals.org/content/230/3/678.short

Worley, B., \& Powers, R. (2013). Multivariate Analysis in Metabolomics. Current Metabolomics, 1(1), 92-107. https://doi.org/10.2174/2213235X11301010092

Wyvell, C. L., \& Berridge, K. C. (2001). Incentive sensitization by previous amphetamine exposure: increased cue-triggered \&quot; wanting\&quot; for sucrose reward. The Journal of Neuroscience: The Official Journal of the Society for Neuroscience, 21(19), 78317840. https://doi.org/10.1523/JNEUROSCI.21-19-07831.2001

Xu, Y.-F., Lu, W., \& Rabinowitz, J. D. (2015). Avoiding misannotation of in-source fragmentation products as cellular metabolites in liquid chromatography-mass spectrometry-based metabolomics. Analytical Chemistry, 87(4), 2273-2281. 
https://doi.org/10.1021/ac504118y

Yeh, S. Y., \& Hsu, F.-L. (1991). The neurochemical and stimulatory effects of putative metabolites of 3,4-methylenedioxyamphetamine and 3,4methylenedioxymethamphetamine in rats. Pharmacology Biochemistry and Behavior, 39(3), 787-790. https://doi.org/10.1016/0091-3057(91)90165-X

Yen, C.-F., \& Hsu, S.-Y. (2007). Symptoms of ecstasy dependence and correlation with psychopathology in Taiwanese adolescents. The Journal of Nervous and Mental Disease, 195(10), 866-869. https://doi.org/10.1097/NMD.0b013e3181568625

Yin, H. H., \& Knowlton, B. J. (2006). The role of the basal ganglia in habit formation. Nature Reviews Neuroscience, 7(6), 464-476. https://doi.org/10.1038/nrn1919

Yin, H. H., Knowlton, B. J., \& Balleine, B. W. (2004). Lesions of dorsolateral striatum preserve outcome expectancy but disrupt habit formation in instrumental learning. European Journal of Neuroscience, 19(1), 181-189. https://doi.org/10.1111/j.14609568.2004.03095.x

Yin, H. H., Ostlund, S. B., Knowlton, B. J., \& Balleine, B. W. (2005). The role of the dorsomedial striatum in instrumental conditioning. European Journal of Neuroscience, 22(2), 513-523. https://doi.org/10.1111/j.1460-9568.2005.04218.x

Yokel, R. A., \& Pickens, R. (1973). Self-administration of optical isomers of amphetamine and methylamphetamine by rats. Journal of Pharmacology and Experimental Therapeutics, 187(1), 27-33.

Yokel, R. A., \& Wise, R. A. (1976). Attenuation of intravenous amphetamine reinforcement by central dopamine blockade in rats. Psychopharmacology, 48(3), 311-318. https://doi.org/10.1007/BF00496868

Yokel, R. A., \& Wise, R. A. (1978). Amphetamine-type reinforcement by dopaminergic agonists in the rat. Psychopharmacology, 58(3), 289-296. https://doi.org/10.1007/BF00427393

Young, A. M. ., \& Rees, K. R. (1998). Dopamine release in the amygdaloid complex of the rat, studied by brain microdialysis. Neuroscience Letters, 249(1), 49-52. https://doi.org/10.1016/S0304-3940(98)00390-5

Zachariou, V., Bolaños, C. A., Selley, D. E., Theobald, D. E. H., Cassidy, M. P., Kelz, M. B., ... Nestler, E. J. (2006). An essential role for $\Delta$ FosB in the nucleus accumbens in morphine action. Nature Neuroscience, 9(2), 205-211. https://doi.org/10.1038/nn1636

Zaitsu, K., Miyawaki, I., Bando, K., Horie, H., Shima, N., Katagi, M., ... Fukusaki, E. (2014). Metabolic profiling of urine and blood plasma in rat models of drug addiction on the basis of morphine, methamphetamine, and cocaine-induced conditioned place preference. Analytical and Bioanalytical Chemistry, 406(5), 1339-1354. https://doi.org/10.1007/s00216-013-7234-1

Zapata, A., Minney, V. L., \& Shippenberg, T. S. (2010). Shift from Goal-Directed to Habitual Cocaine Seeking after Prolonged Experience in Rats. Journal of Neuroscience, 30(46), 15457-15463. https://doi.org/10.1523/JNEUROSCI.4072-10.2010

Zhang, A., Sun, H., Wang, P., Han, Y., \& Wang, X. (2012). Modern analytical techniques in metabolomics analysis. The Analyst, 137(2), 293-300. https://doi.org/10.1039/C1AN15605E

Zhao, W., \& Becker, J. B. (2010). Sensitization enhances acquisition of cocaine selfadministration in female rats: estradiol further enhances cocaine intake after acquisition. Hormones and Behavior, 58(1), 8-12. https://doi.org/10.1016/j.yhbeh.2009.09.005

Zheng, T., Liu, L., Aa, J., Wang, G., Cao, B., Li, M., ... Zhu, X. (2013). Metabolic phenotype of rats exposed to heroin and potential markers of heroin abuse. Drug and Alcohol Dependence, 127(1-3), 177-186. https://doi.org/10.1016/J.DRUGALCDEP.2012.06.031 Zheng, T., Liu, L., Shi, J., Yu, X., Xiao, W., Sun, R., ... Wang, G. (2014). The metabolic 
impact of methamphetamine on the systemic metabolism of rats and potential markers of methamphetamine abuse. Mol. BioSyst., 10(7), 1968-1977. https://doi.org/10.1039/C4MB00158C

Zhou, B., Xiao, J. F., Tuli, L., \& Ressom, H. W. (2012). LC-MS-based metabolomics. Mol. BioSyst., 8(2), 470-481. https://doi.org/10.1039/C1MB05350G 


\section{Acknowledgements}

First and foremost, I would like to thank my supervisor, Prof. Susan Schenk, for all that you have taught me over the years and for your inspiring passion for science.

I would also like to express my gratitude to my collaborators from the School of Chemical and Physical Sciences, Dr Rob Keyzers and Ian Vorster, whose advice and support was instrumental to the completion of the latter half of this thesis, as well as my secondary supervisor, Dr Maree Hunt.

To the current and previous members of the lab (both human and rodent), a sincere thank you for your crucial contributions and for making my time in the lab more enjoyable. A special thanks to Joyce, who patiently taught me many of my technical skills.

I would also like to thank my family, friends, and most of all, Catherine, I cannot express how truly grateful I am for what has nearly been a decade of your support.

Lastly, I would like to acknowledge the generous funding that was received from the Todd Foundation, the Wellington Medical Research Foundation, the Neurological Foundation, the National Institute of Health, the late Claude McCarthy and the Public Trust, as well as Victoria University of Wellington for research expenses, conference expenses, or for tuition fees and living costs.

Cheers. 